University of San Diego

Digital USD

1998-06-01

\title{
Relationships between Posttraumatic Stress, Acculturation, and Maternal Sensitivity in Vietnamese and Hmong Mothers
}

Gwendolyn F. Foss DNSc

University of San Diego

Follow this and additional works at: https://digital.sandiego.edu/dissertations

Part of the Nursing Commons

\section{Digital USD Citation}

Foss, Gwendolyn F. DNSc, "Relationships between Posttraumatic Stress, Acculturation, and Maternal Sensitivity in Vietnamese and Hmong Mothers" (1998). Dissertations. 276.

https://digital.sandiego.edu/dissertations/276

This Dissertation: Open Access is brought to you for free and open access by the Theses and Dissertations at Digital USD. It has been accepted for inclusion in Dissertations by an authorized administrator of Digital USD. For more information, please contact digital@sandiego.edu. 


\title{
RELATIONSHIPS BETWEEN POSTTRAUMATIC STRESS, ACCULTURATION, AND MATERNAL SENSITTVITY IN VIETNAMESE AND
} HMONG MOTHERS

\author{
by \\ Gwendolyn F. Foss \\ A dissertation presented to the \\ FACULTY OF THE PHILIP Y. HAHN SCHOOL OF NURSING \\ UNIVERSITY OF SAN DIEGO
}

\author{
In partial fulfillment of the \\ requirements for the degree \\ DOCTOR OF NURSING SCIENCE
}

June, 1998 


\section{Copyright 1998 Gwendolyn F. Foss}




\begin{abstract}
The purposes of this study were to determine if posturaumatic stress (PTS), depression, and anxiety occurred in a community sample of Vietnamese and Hmong mothers and to describe relationships between PTS, depression, anxiety, acculturation and maternal sensitivity. Transition theory (Bridges, 1980), and a conceptual model of parenting in immigrant populations building on Belsky's (1984) work, provided the theoretical framework (Foss, 1996).

The sample was divided evenly between Vietnamese and Hmong participants. Ages ranged from 17-43 years, time lived in the United States ranged from 3-21 years, and education ranged from no formal education to completion of college. Maternal sensitivity was measured with Ainsworth's Sensitivity vs. Insensitivity to the Communication of the Baby Scale; PTS, depression, and anxiety with the Vietnamese and Hmong versions of the Hopkins Symptom Checklist-25 (HSCL-25), and acculturation with the Suinn-Lew Selfidentity Acculturation Scale (SL-ASIA). The SL-ASIA was translated into Vietnamese and Hmong prior to data collection. All data were collected by the investigator in the home. Interpreters were used for half of the sample.

The Statistical Package for the Social Sciences 6.1 (SPSS) was used to analyze the data, explore relationships, and perform regression analyses. Results were (a) Vietnamese mothers experience PTS, anxiety, and depression to the same degree as the general Vietnamese and Hmong population, (b) Hmong mothers experience PTS, depression and anxiety to a much greater degree than Vietnamese mothers, (c) maternal sensitivity remained very high, even when clinical levels of PTS, anxiety, or depression were present, (d) there was a trend for more depressed Vietnamese mothers to be less sensitive to their infants, but for more depressed Hmong mothers to be more sensitive to their infants, and (e) more acculturated mothers tended to be less sensitive mothers. Further analyses revealed that the number of years spent in transit from the homeland to the United States was associated with greater matemal sensitivity, especially in the Hmong group; lower maternal sensitivity was associated with a greater number of the husband's family living in the United States, and having more pregnancies was strongly associated with greater depression. Recommendations for research and practice were offered.
\end{abstract}




\section{ACKNOWLEDGEMENTS}

Completion of this program of study would not have been possible without considerable help from many people. Space does not permit a full accounting of all those who so generously and enthusiastically supported me throughout this process; however, I would like to acknowledge and thank a few people here.

I am deeply grateful to Dr. Colette Jones, my academic advisor and dissertation chair, for her constant support, encouragement, advice, and availability. From the beginning she was an enthusiastic mentor whose insights and contributions helped form my own ideas and were essential to my success. I am also deeply grateful to Dr. Diane Hatton and Dr. Mary Abascal-Hildebrandt who provided valuable comments prior to collection of my research data as well as during the writing phase. I am extremely grateful for their continued participation in my dissertation, and for returning to San Diego from their new residences to see this project through to completion at the dissertation defense.

I am grateful to Dr. Janet Rodgers, the dean, and the faculty of the Philip Y. Hahn School of Nursing for fostering a stimulating learning environment. Special thanks go to those faculty with whom I studied in formal courses, and to faculty in other disciplines who provided expertise for very specific independent studies. All were uniformly supportive of a learning program that did not always fit into conventional structures, and all were generous with their expertise and time. I am much richer for having studied with them. In particular I would like to acknowledge and thank the office staff at the School of Nursing for their consistently cheery and knowledgeable answers to myriads of questions I posed. I am especially grateful to the librarians who requested and obtained so many necessary resources from a wide variety of libraries. Doing background research for this dissertation was made much easier because they were available.

It was due to the initial support and encouragement of Dr. Margaret Stevenson and Dr. Rosemary Goodyear that I began a doctoral educational program at the University of San Diego. That support continued in many tangible ways throughout my program of 
studies and I heartily thank them for their belief in me. I wish to thank my classmates for all the times we spent exchanging ideas, having fun, and learning from each other. Those experiences were rewarding and enriched my time at the University. Finally, completion of this program was made much easier by the constant support and encouragement of my husband Charlie, and our children, Sandy and Lynne. We had fun and learned from each other. Thank you. 


\section{TABLE OF CONTENTS}

ABSTRACT

ACKNOWLEDGEMENTS $\ldots \ldots \ldots \ldots \ldots \ldots \ldots \ldots \ldots \ldots \ldots$ ii

TABLE OF CONTENTS $\ldots \ldots \ldots \ldots \ldots \ldots \ldots \ldots \ldots \ldots \ldots \ldots$ iv

LIST OF TABLES $\ldots \ldots \ldots \ldots \ldots \ldots \ldots \ldots \ldots \ldots \ldots \ldots$

LIST OF FIGURES $\ldots \ldots \ldots \ldots \ldots \ldots \ldots \ldots \ldots \ldots \ldots$ viii

LIST OF APPENDICES $\ldots \ldots \ldots \ldots \ldots \ldots \ldots \ldots \ldots \ldots \ldots \ldots$

CHAPTER 1 INTRODUCTION $\ldots \ldots \ldots \ldots \ldots \ldots \ldots \ldots \ldots \ldots \ldots \ldots$

The Problem $\ldots \ldots \ldots \ldots \ldots \ldots \ldots \ldots \ldots \ldots \ldots \ldots$

Purpose of the Study $\ldots \ldots \ldots \ldots \ldots \ldots \ldots \ldots \ldots \ldots \ldots$

Conceptual Framework $\ldots \ldots \ldots \ldots \ldots \ldots \ldots \ldots \ldots \ldots \ldots \ldots \ldots \ldots$

Research Questions ......................... 7

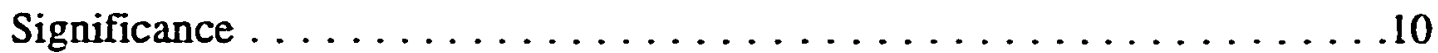

Operational Definitions $\ldots \ldots \ldots \ldots \ldots \ldots \ldots \ldots \ldots \ldots \ldots \ldots \ldots \ldots$

CHAPTER 2 REVIEW OF THE LITERATURE $\ldots \ldots \ldots \ldots \ldots \ldots \ldots \ldots$

Conceptual Framework $\ldots \ldots \ldots \ldots \ldots \ldots \ldots \ldots \ldots \ldots \ldots \ldots$

Transition. ....................... 15

Transition to Parenthood. . ............... 18

Migration . .......................

Determinants of Parenting $\ldots \ldots \ldots \ldots \ldots \ldots \ldots \ldots$

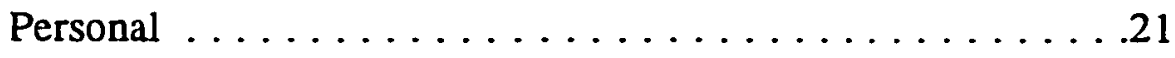

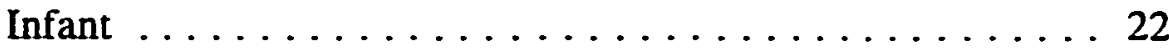

Contextual ...................... 23

History. . . . . . . . . . . . . . . . . . . 27

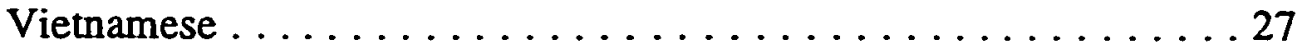

Hmong . . . . ....................... 29

The Vietnam War .................... 31

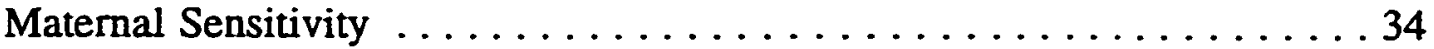

Posttraumatic Stress . . . . . . . . . . . . . . . . . 38

iv 


\section{TABLE OF CONTENTS (Continued)}

Acculturation . . . . . . . . . . . . . . . . . 43

Acculturation of Vietnamese and Hmong Peoples ...........43

Acculturation and Health Status . . . . . . . . . . . . 45

Acculturative Stress . . . . . . . . . . . . . . . . 47

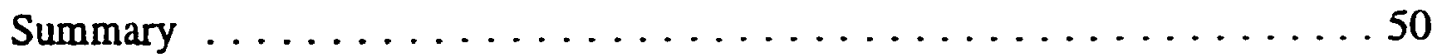

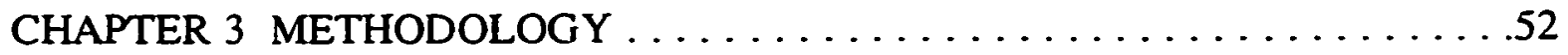

Sampling Procedure $\ldots \ldots \ldots \ldots \ldots \ldots \ldots \ldots \ldots \ldots \ldots \ldots \ldots \ldots \ldots \ldots \ldots$

Entree into the community . . . . . . . . . . . . . 52

Vietnamese Participants . . . . . . . . . . . 53

Hmong Participants . . . . . . . . . . . . 54

Description of the Sample .................. 54

Sample Size .......................... 55

Research Design . . . . . . . . . . . . . . . . 56

Use of Interpreters $\ldots \ldots \ldots \ldots \ldots \ldots \ldots \ldots \ldots \ldots$

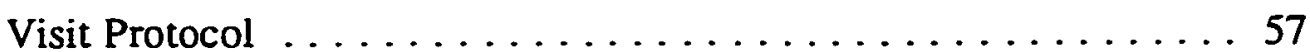

Protection of Human Subjects $\ldots \ldots \ldots \ldots \ldots \ldots \ldots \ldots . \ldots \ldots$

Instruments $\ldots \ldots \ldots \ldots \ldots \ldots \ldots \ldots \ldots \ldots \ldots \ldots \ldots \ldots \ldots \ldots \ldots \ldots$

Cross-cultural Instrumentation $\ldots \ldots \ldots \ldots \ldots \ldots \ldots 6 . \ldots \ldots$

Translation Protocol . . . . . . . . . . . . . 62

Translation of Flyers and Consent .............6 63

Demographic Data .......................... 64

Matemal Sensitivity . . . . . . . . . . . . . . . 64

Suinn-Lew Self-identity Acculturation Scale (SL-ASIA) . . . . . . 66

Hopkins Symptom Checklist-25 (HSCL-25) . . . . . . . . . . 69

Analysis of Data . . . . . . . . . . . . 72

Assumptions and Limitations $\ldots \ldots \ldots \ldots \ldots \ldots \ldots \ldots \ldots \ldots \ldots \ldots \ldots \ldots$

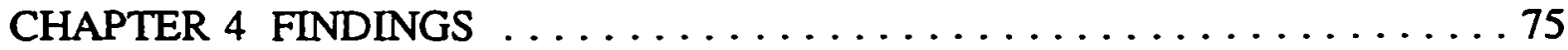

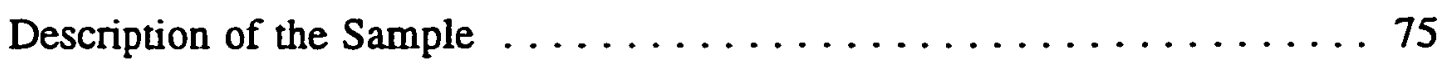




\section{TABLE OF CONTENTS (Continued)}

Research Question One . . . . . . . . . . . . . . . . 77

Research Question Two $\ldots \ldots \ldots \ldots \ldots \ldots \ldots \ldots \ldots \ldots$

Research Question Three $\ldots \ldots \ldots \ldots \ldots \ldots \ldots \ldots \ldots \ldots$

Research Question Four $\ldots \ldots \ldots \ldots \ldots \ldots \ldots \ldots \ldots$

Other Findings $\ldots \ldots \ldots \ldots \ldots \ldots \ldots \ldots \ldots \ldots \ldots \ldots \ldots$

Summary $\ldots \ldots \ldots \ldots \ldots \ldots \ldots \ldots \ldots \ldots \ldots \ldots \ldots \ldots \ldots \ldots \ldots$

CHAPTER 5 DISCUSSION OF THE FINDINGS $\ldots \ldots \ldots \ldots \ldots \ldots \ldots 94$

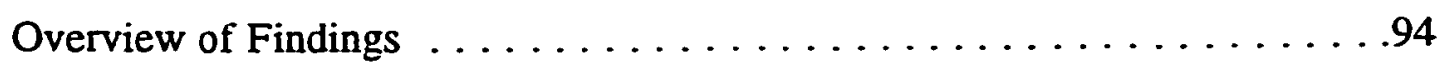

Results . . . . . . . . . . . . . . . . . .94

Methodology . . . . . . . . . . . . . . . . . .96

Research Question One $\ldots \ldots \ldots \ldots \ldots \ldots \ldots \ldots \ldots \ldots \ldots$

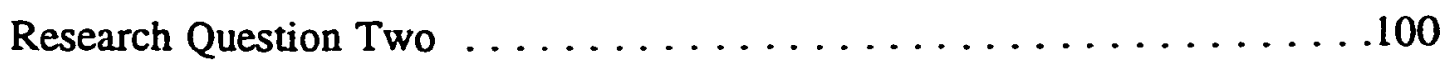

Research Question Three ........................ 103

Research Question Four . . . . . . . . . . . . . . . . 104

Other Findings $\ldots \ldots \ldots \ldots \ldots \ldots \ldots \ldots \ldots \ldots \ldots \ldots \ldots \ldots$

Conclusions ................................. 109

Recommendations ..................... 110

Future Research . . . . . . . . . . . . . . . . . 110

Nursing Practice $\ldots \ldots \ldots \ldots \ldots \ldots \ldots \ldots \ldots \ldots \ldots \ldots \ldots \ldots \ldots \ldots$

Summary . . . . . . . . . . . . . . . . . . . . 113

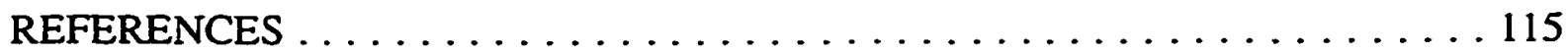

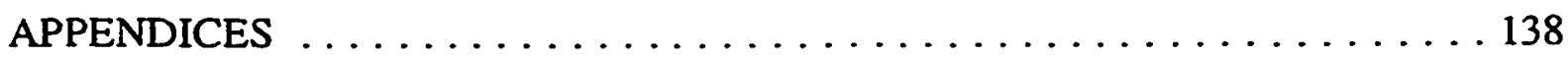

vi 


\section{LIST OF TABLES}

Table 1

Contextual Determinants ..................... 24

Table 2

Education, Occupation, and Religion of Participants by Ethnic Group . . . . 76

Table 3

Mean, Range, and Standard Deviation of HSCL-25, Acculturation, and

Maternal Sensitivity Scores . . . . . . . . . . . . . . . . . 81

Table 4

Correlation Coefficients ( $r$ ) of Predictor and Demographic Variable for

Combined Groups . . . . . . . . . . . . . . . . . . . . 82

Table 5

Correlation Coefficients $(r)$ of Predictor and Demographic Variable for

Vietnamese Groups . . . . . . . . . . . . . . . . . . . . . 83

Table 6

Correlation Coefficients ( $r$ ) of Predictor and Demographic Variable for

Hmong Groups . . . ......................... 84

Table 7

Relationships of PTS, Depression, and Anxiety ........... 85

Table 8

Relationships of Acculturation, Depression, and Anxiety . . . . . . . . 89

Table 9

Relationships of Matemal Sensitivity, Age, Number of Years in Transition, and Number of Husband's Family in the United States . . . . . . . . . . . 92

vii 


\section{LIST OF FIGURES}

Figure 1

Determinants of Parenting in Immigrant Populations $\ldots \ldots \ldots \ldots \ldots \ldots$

Figure 2

Relationships of Predictor Variables to Maternal Sensitivity . . . . . . . . 9

Figure 3

Transition and Parenting in Immigrant Populations ........... 17

Figure 4

Comparison of Depression and Anxiety by Ethnicity . . . . . . . . 86 Figure 5

Specified Relationships of Predictor Variables to Maternal Sensitivity . . . . 95 


\section{LIST OF APPENDICES}

Appendix A

Letters of Permission. . . . . . . . . . . . . . . . 138 Appendix B

Recruitment Flyers . . . . . . . . . . . . . . . . . . . 144 Appendix C

Consent for Participation $\ldots \ldots \ldots \ldots \ldots \ldots \ldots \ldots \ldots$ Appendix D

Demographic and Misc. Data ................... 156 Appendix E

Sensitivity Versus Insensitivity to the Baby's Communications Scale with Scale Description ... . . . . . . . . . . . . . . . . . . . . 159 Appendix F

Suinn-Lew Asian Self-identity Acculturation Scale (SL-ASIA) . . . . . 166 Appendix $G$

Hopkins Symptom Checklist-25 . . . . . . . . . . . . . 201 Appendix $\mathrm{H}$

Health Education Materials from the Department of Health Services . . . . 210 Appendix I

University of San Diego Committee on the Protection of Human Subjects . . 239 Appendix J

Results of t-tests for Differences between Vietnamese and Hmong Groups . . 242 


\title{
RELATIONSHIPS BETWEEN POSTTRAUMATIC STRESS, ACCULTURATION, AND MATERNAL SENSITIVITY IN VIETNAMESE AND HMONG MOTHERS
}

\author{
CHAPTER 1
}

Introduction

The ability of a mother to provide for the needs of her infant is an ongoing concern of health protessionals in the United States. If infants do not receive consistent nurturing, they do not thrive. Maternal sensitivity is the term that refers to the the "mother's ability to perceive and to interpret accurately the signals and communications implicit in her infant's behavior, ... [and] to respond .... appropriately and promptly" (Ainsworth, Bell \& Stayton, 1974, p. 127). Lack of a sensitive maternal response, such as that resulting from depression in the mother, has been shown to place infants at a higher risk for failure to thrive, developmental delays, and for infant neglect and abuse (Bowlby, 1965; Field, 1995; Susman, Trickett, Iannotti, Hollenbeck, \& Zahn-Waxler, 1985), while sensitive parenting has been related to securely attached infants (Ainsworth, et al., 1974), better school performance, and better socialization skills at grade six (Pettit, Bates, \& Dodge, 1997).

Maternal sensitivity has been described in many different nations in similar ways, but the studies have focused on resident citizens. Maternal sensitivity in immigrants, people who have moved to a different country (Heisler, 1992), has been neglected. Until 1900, 97\% of all immigrants came to the United States from Europe, and predominantly Northern Europe (Carlson, 1994). Since then, immigrants have come from increasingly diverse parts of the globe. Now about one million immigrants arrive in the United States each year (Martin \& Midgley, 1994), either hoping to seek a better life for themselves or because they 
This later group of immigrants has been categorized as "refugees" (Muecke, 1992).

After the Vietnam war, refugees from Southeast Asia streamed to the United States in search of safety. They had been exposed to particularly traumatic situations before and during their journey (Gold, 1992; Hamilton-Merritt, 1993). After arriving in the United States, they had to begin the arduous and extremely stressful task of learning about their new society and making the transition to living in a new environment quite different from that of earlier locales. It is not surprising that a high incidence of Posttraumatic Stress Disorder (PTSD) and depression has continued to be extensively documented in that population (Abueg \& Chun, 1996; Chung \& Kagawa-Singer, 1993; Hauff \& Vaglum, 1995; Nicholson, 1997). For example, Nicholson, in a recent study (1997) found that $40 \%$ of her community sample of Southeast Asians in the United States suffered from depression, 35\% from anxiety, and 14\% from PTSD. Both premigration and postmigration stressors contributed to those findings. The majority of other studies that were reviewed indicated that acculturation was a major post-migration stressor, and was labeled acculturative stress. For this reason, it was important to examine also if or how the difficult process of acculturation is related to matemal sensitivity.

The medical diagnosis of PTSD is restricted to the specific conditions given in the Diagnostic and Statistical Manual of Mental Disorders, Fourth, Edition (DSM-IV) (American Psychiatric Association, 1994, cited in Marsella, Smith, Murphy, \& Scurfield, 1996). In a large community sample, a lifetime PTSD rate of $0.5 \%$ in men and $1.3 \%$ in women has been found; however, $15 \%$ of men and $16 \%$ of women have experienced some symptoms after a traumatic event (De Girolamo \& McFarlane, 1996). In the refugee community, reviewed studies focused on the epidemiology of PTSD in various cultural groups and on identifying effective treatment modalities for PTSD. That body of literature did not discuss apparently healthy community samples of refugee mothers of young infants. The present study used the term "posttraumatic stress" (PTS) rather than PTSD to reflect a broader nonmedical focus that did not emphasize the medically distinct diagnosis of PTSD. PTS has been defined as a "diverse myriad of stress-related physical complaints and disorders in addition to or in concomitance with ... psychiatric and behavioral disorders" that can be manifested as depression, anxiety or somatization (Everly, 1995, p. 
7). It does not require adherence to the American Psychiatric Association's definition (Marsella, et al., 1996, pp 31-32).

Upon arrival, many refugees need assistance establishing a network of health care providers. Public health nurses often are the ones who assist them to locate appropriate resources. While practicing as a public health nurse, the author had extensive experience working with many different refugee populations, among them many Southeast Asians. During that time she became aware of the broad spectrum of education and lifeways represented by the Vietnamese and Hmong people when they came to the United States. She believed that findings from a study that used samples from these two very different groups would provide a broader insight into phenomena of interest and provide a better guide for future research among various groups of refugees than would be provided by a study of just one group. For that reason this study focused on both Vietnamese and Hmong families.

Vietnamese and Hmong people share many commonalities. Alone among all the peoples of Southeast Asia, they were subjugated by China, with the result that Chinese ethics, values, family structures, and belief systems continue to be held by both groups (Luong, 1984; Quincy, 1995). Both suffered greatly as a result of their alliances with the French and the United States during the 20th century (Gold,1992; Hamilton-Merritt, 1993). Before migration significant differences existed between them. While the Vietnamese people had broad access to schools (Jamieson, 1993), the Hmong had no schools available to them until after World War II, when missionaries began to establish scattered schools. Eventually, government schools did become available to many boys, but only rarely to girls (Quincy, 1995). Hence the level of education differed greatly between the Vietnamese and Hmong. Vietnamese citizens participated in all activities of society, from national government to all branches of the military to farm workers (Jamieson, 1993), while the Hmong were restricted to farming and to the lowest levels of military rank (Bamey, 1979; Quincy, 1995). This disparate history has contributed to differences and similarities in how each group continues to negotiate the transition to life in the United States.

Chapter one discusses the problem, the purpose of the study, the conceptual 
framework, the research questions, and the significance of the study. A list giving the definitions of terms is included at the end of the chapter.

\section{The Problem}

In a 1951 monograph for the United Nations, john Bowlby (1952; 1965) compellingly described how growth retardation, developmental delays or disabilities, and personality disorders were linked to the lack of a mother's nurturing love. Ainsworth (1967) established how a mother's sensitive response to her infant's cues provided a secure environment that allowed an infant to thrive. Maternal sensitivity has been shown to predict of the security of the infant in strange situations (Ainsworth,et al., 1974). While the notion of the mother functioning as an attachment figure and as a secure base for an infant or child seems to be widely supported in all cultural groups investigated to date, how that secure base is used to mold the child to culturally acceptable roles varies (Posada. et al., 1995). What is not known is what happens to maternal sensitivity when the mother is coping with all the changes that accompany transition to an alien society. When matemal sensitivity among Hmong mothers has been examined, the focus has only been on comparing Hmong and European-American populations (Muret-Wagstaff \& Moore, 1989; Oberg, Muret-Wagstaff, Moore, \& Cumming, 1986). No studies of maternal sensitivity in Vietnamese mothers were located.

Building on the base Bowlby and Ainsworth established, a variety of programs have been developed to support the development of positive parenting practices in families identified by health care professionals as high risk families. An implicit assumption by practitioners and researchers alike has been that "good parenting" is the same in all cultures, thus readily observed by professional health care workers (Foss, 1995; Gross, 1996; Vondra, Shaw, Kevenides, 1995). Often immigrant parents are unaware that their indigenous parenting patterns are different from those extant in the dominant society. Frequently they do not speak English and are unable to articulate their parenting expectations and patterns to health care professionals. When professionals consider parenting skills weak or absent, they usually offer immigrant parents the identical education and support offered to parents from dominent cultures. All too often the result has been 
inappropriate and insensitive intervention by health care protessionals (Foss, 1996; Jackson, 1993).

Criticism of an assumption of universal parenting patterns in all populations has begun to appear in the literature (Foss, 1996; Gross, 1996: Harwood, Miller, \& Irizarry, 1995). As a result, there have been increasing numbers of studies addressing differences and similarities in mother-infant interactions occurring in different nations and cultural groups (Harkness \& Super, 1996; MacPhee, Fritz, \& Miller-Heyl, 1996). Again, these studies have focused simply on comparing ethnic groups. How the process of transition to a new society influences mother-infant interactions has not been addressed in the literature that was reviewed.

As stated previously, extensive research has been conducted to determine the extent of PTSD, anxiety, and depression, as well as optimal treatment patterns for various refugee populations. No research studies were found that questioned the presence or absence of PTSD, depression, or anxiety in Hmong or Vietnamese mothers, nor did any studies question if maternal sensitivity was altered in the presence of PTS, PTSD, depression, or anxiety. In summary, three major problems were identified.

1. There is little known about maternal sensitivity in groups who are coping with transition to a different society.

2. There is scant information about the incidence of posttraumatic stress, depression, and anxiety in Vietnamese and Hmong mothers.

3. No information has been located about influences from the above negative factors on maternal sensitivity in mothers from Southeast Asia who have migrated to the United States or other countries.

\section{Purpose of the Study}

The purposes of this study were to (a) determine if posttraumatic stress, depression, and anxiety occurred in a community sample of Vietnamese and Hmong mothers of infants and (b) describe relationships between posttraumatic stress, depression, anxiety, acculturation and matermal sensitivity. Only Vietnamese and Hmong families who immigrated since 1975 were included. 
This purpose was consistent with the need outlined in Healthy People 2000 (United States Public Health Service, 1992). That document listed many objectives related to improving the health of vulnerable populations, including mothers of infants. Three objectives that relate specifically to this study have been listed here.

6.5 "Reduce to less than 35\% the proportion of people aged 18 and older who experienced adverse health effects from stress within the past year" (p. 214).

6.8 "Increase to at least $20 \%$ the proportion of people aged 18 and older who seek help in coping with personal and emotional problems (p. 216).

8.11 "Increase to at least 50\% the proportion of counties that have established culturally and linguistically appropriate community health promotion programs for racial and ethnic minority populations" (p. 601)

If depression, anxiety, and postrraumatic stress are present in greater degrees amongVietnamese and Hmong mothers of young infants, health care professionals need to be aware of that tendancy. Only then can support systems be designed and offered to mothers experiencing this kind of pain and distress. In this way additional progress can be made toward achieving the identified goals of Healthy People 2000.

\section{Conceptual Framework}

Transition theory (Bridges, 1980) and Belsky's determinants of parenting (1984) provided the conceptual framework for this study. Bridges defined the three stages of transition as endings, a neutral zone, and beginnings. Endings refers to the termination of something familiar, the neutral zone is that time between the old and the new, and beginnings is the start of something new. Schumacher and Meleis (1994) clearly articulated how use of the concept of transition provided a valuable framework to examine patterns of human responses to external events or internal changes. Although he did not use the term, Berry's (1990) discussion of acculturation illustrated these stages of transition.

Determinants of positive parenting were identified by Belsky (1984) as the characteristics of the parent, characteristics of the infant, and the context surrounding both. He considered the parental personality to contribute the most toward the establishment and maintenance of an interaction pattern between parent and infant, and considered the context 
to contribute the least. A conceptual model of parenting in immigrant populations that built on Belsky's work was proposed by Foss (1996), and is included with the permission (Appendix A) of Advances in Nursing Science, as Figure 1. In that article Belsky's original categories of personal, infant and contextual determinants were kept; however, the variables of each category were expanded or changed to reflect the experiences of immigrants. The model shows the interaction of the three determinants of parenting, and their influence on maternal sensitivity. Contextual characteristics of parenting reflecting experiences of immigrants included premigration and migration, post-migration, ethnicity, and sociopolitical and environmental factors. Maternal sensitivity thus reflected influences of transition both to parenthood and to a new society. A part of that model was tested in the present study.

\section{Research Questions}

The following research questions were addressed in this study.

1. To what extent do mothers in Vietnamese and Hmong populations experience posttraumatic stress (PTS)?

2. To what extent does PTS, anxiety, or depression decrease maternal sensitivity in Vietnamese and Hmong mothers?

3. To what extent does acculturation decrease maternal sensitivity in Vietnamese and Hmong mothers?

4. Among Vietnamese and Hmong mothers, is there a relationship between PTS, or its components of depression and anxiety, and acculturation?

A descriptive correlational (Burns \& Grove, 1993) research design was used. The theoretical relationships between the variables shown in Figure 2 were tested. Anxiety and depression are shown as domains of PTS. (PTS is present when the combined score of depression and anxiety reaches clinical levels.) In the model, independent or exogenous variables were posttraumatic stress and acculturation. The endogenous or dependent variable was maternal sensitivity. Anxiety, depression, (or PTS) and acculturation were 
Figure 1

Determinants of Parenting in Immigrant Populations

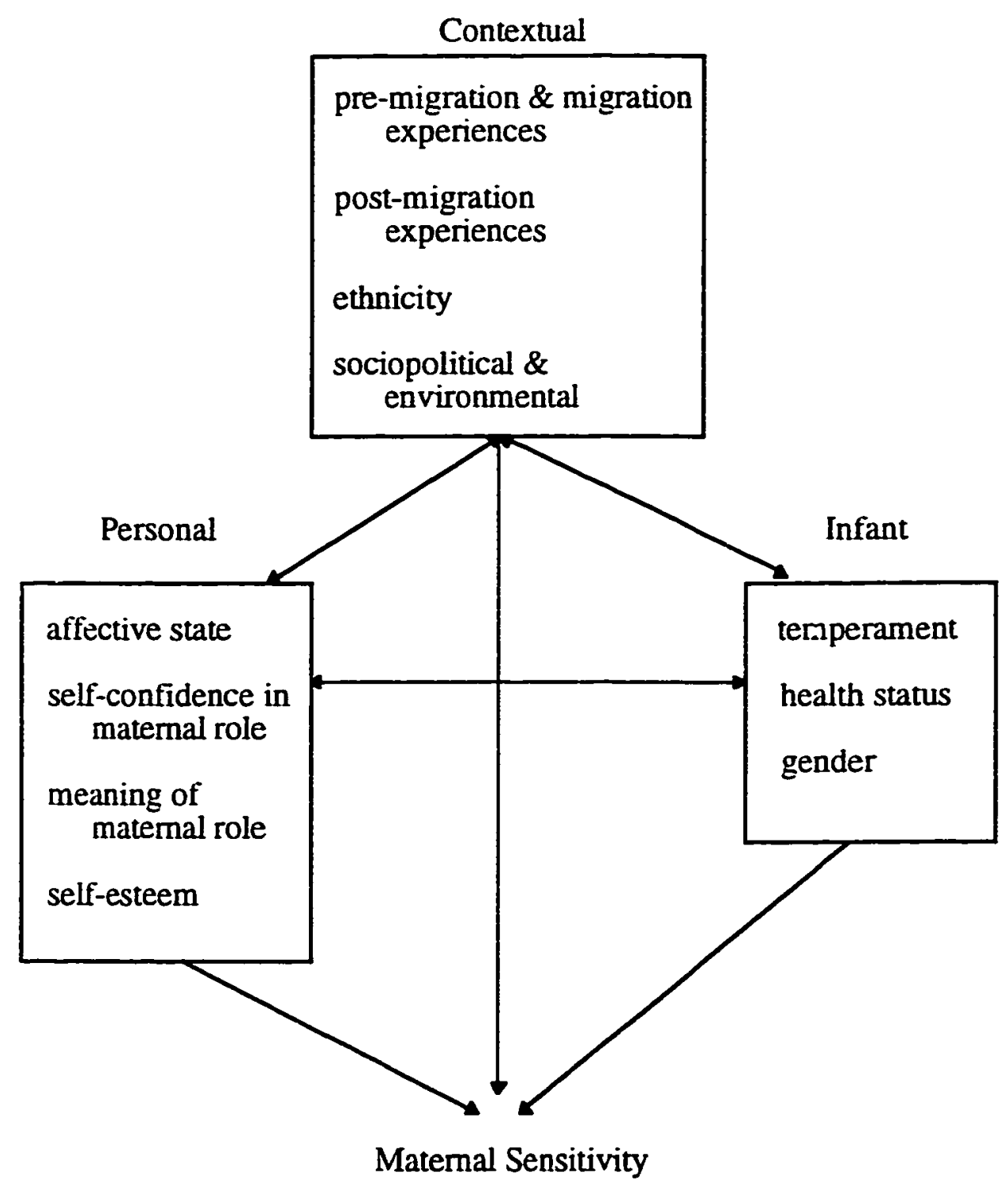

Modified with permission from Foss, G. F. (1996). A conceptual model for studying parenting in immigrant populations. Advances in Nursing Science.19(2), 80. Copyright 1996 Aspen Publishers, Inc. 
Figure 2

Relationships of Predictor Variables to Maternal Sensitivity

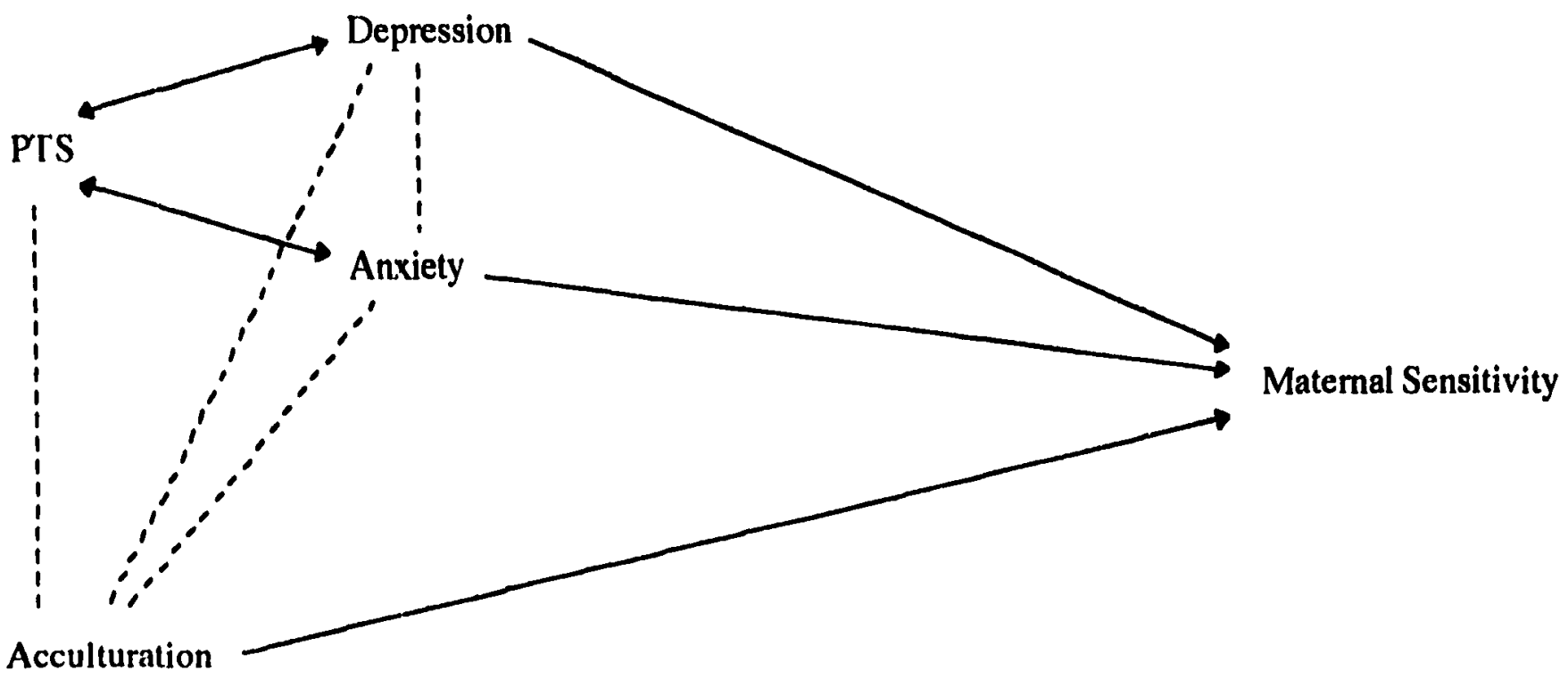

PIS = Posttraumatic Stress 
predicted to decrease maternal sensitivity. Interrelationships among PTS, depression, anxiety and acculturation are shown by dotted lines.

\section{Significance}

The number of immigrants in the United States has been increasing. Between 1971 and 1993, 15.5 million new immigrants legally entered (Martin \& Midgley, 1994). About $10 \%$ entered as refugees (Bureau of Population, Refugees, \& Migration, cited in U.S. Committee for Refugees, 1996, p. 10-11). Fully one third of all immigrants in the United States live in California (Martin \& Midgley, 1994). Furthermore, in 1990, 22\% of all California residents were born in another country (U.S. Bureau of the Census, 1994).

Since $1990,91 \%$ of the population growth of California has been due mostly to immigration of Hispanics and Asian/Pacific Islanders (State of Califormia, Dept of Finance, 1998). California continues to be a magnet for immigrants from Asia, many of whom stay permanently. The percentage of the tota! population of California which is AsianAmerican/Pacific Islanders has risen from 9\% in 1995 to $11 \%$ in mid-1996 (State of California, Department of Finance, 1998). In 1995, 40\% of all Asian-Americans lived in California (Massey, 1995).

The city of San Diego is the destination of choice for many immigrants. The majority have come from Mexico, but great numbers have come from Asia. In 1996, 13\% of the population of the city of San Diego identified itself as Asian, reflecting a 41\% increase in the number of Asian residents in San Diego since the 1990 census (SANDAG/Sourcepoint, 1997). Other parts of the nation have also reported a rapid increase in immigrants. For example, the Charlotte Observer reported that more than 75,000 people from at least 29 countries lived in the Charlotte area of North Carolina in 1995 (Hopkins, March 24, 1997). One year later, the same paper reported at least 100,000 immigrants lived in that region in 1996 (Wahl, February 15, 1998).

Immigrant women are young and have a fertility rate almost double that of U.S.born women (De Vita, 1996). In 1990, although foreign-born residents accounted for $8 \%$ of the total population, they accounted for $15 \%$ of total births (Martin \& Midgley, 1994). 
This same trend was reflected in the 1995 birth rates for California. The birth rate (per thousand) for Whites was 11.2, for African-Americans, 17.1, for Hispanics, 26.0, and for Asians, Pacific Islanders, Native Americans and others, 17.5 (Ficenec, 1996). In 1996, 9.93\% of all births in California were to mothers of Asian origin. Of that percentage, $16.65 \%$ were to Vietnamese and Laotian mothers. San Diego reflected California patterns with $9.6 \%$ of all births occurring to Asian mothers. Of all births to Asian mothers in San Diego, a total of $16.46 \%$, or one out of six births to women of Asian heritage, were to Vietnamese and Laotian women (California Department of Health Services, 1998). The need for health care providers to understand the health care needs of this particular group of vulnerable mothers appears relavant.

When refugees arrive, they usually are destitute, poorly nourished, and in poor health as a result of their premigration and migration experiences (Muecke, 1992). Often they do not speak the local language. They encounter customs that are very different from their own, and they are faced with health care providers who do not understand their lifeways, or the magnitude of their traumatic migration experiences (Cole, Espin, \& Rothblum, 1992; Meleis 1991). While PTSD, anxiety and depression have been widely identified in all immigrants, refugee and non-refugee alike, they have not been specifically identified in refugee mothers (Chung \& Kagawa-Singer, 1993; Juthani, 1992; Nicholson, 1997; Tran, 1993). Nicholson's (1997) study found that premigration, migration, and post-migration events predicted the incidence and severity of PTSD in Southeast Asian refugees. There were equal numbers of women and men in her sample. The mean age of the women was 42 , but the parity or number of children was not reported. With findings of widespread PTSD, depression and anxiety continuing to be found in large nonclinical and gender-balanced samples of Southeast Asians, the need is compelling to examine if there are high levels of stress reported in Southeast Asian community samples of mothers of infants. If there is posttraumatic stress, depression, or anxiety in refugee mothers, it is essential to determine how this influences maternal sensitivity to an infant. Then health care that is both sensitive to their experience and accessible can be offered.

How acculturation is related to maternal sensitivity and, thus, to infant health is unclear. Infant mortality rates for new Indochinese immigrants in San Diego were found to 
be lower than the overall rate for the county (Board on Children and Families, 1995). Acculturation has been positively related to lower birth weight in infants born to Latina women (Cobas, Balcagas, Benin, Keith \& Chong, 1996) but not in Southeast Asian women ( $\mathrm{Li}$, et al, 1990). In both instances, these findings were related to the use of street drugs, alcohol and cigarettes during pregnancy. Matemal sensitivity to the infant was not investigated.

The Institute of Medicine and others have called for research specifically on immigrant infants and children so that more appropriate health care educational programs and social policy can be established (Board on Children and Families, et al., 1995). Preventing health problems in families with infants and young children by influencing policy development has been a strategy of community health nurses since the time of Lillian Wald (Buhler-Wilkerson, 1993). As was the case one hundred years ago, nurses again are called upon to assist in policy development on behalf of those who have no voice at the policy-making level. It is impossible to be an effective advocate for vulnerable populations when the full extent of the problems and issues are not understood. With the increasing shift of health care to the community, and with increasing numbers of new young immigrants, research-based knowledge about relationships of posttraumatic stress, depression, anxiety, acculturation, and maternal sensitivity in various immigrant populations is greatly needed. This study expands the knowledge base about mothers with young infants who have experienced the overlapping transitions of immigration and transition to parenthood.

\section{Qperational Definitions}

Acculturation: "culture change that is initiated by the conjunction of two or more autonomous cultural systems. ... [and] results in population-level changes that are due to contact with other cultures" (Social Science Research Council, 1954, cited by Berry, 1990, p. 233-234).

Acculturative Stress: stress due to the acculturation process that results in "anxiety, depression, feelings of marginality and alienation, heightened psychosomatic symptoms, and identity confusion" (Williams \& Berry, 1991, p. 634). 
Attachment: " an affectional tie that one person forms to another specific person, binding them together in space and enduring over time" (Ainsworth, 1973, p. 1).

Culture: "the learned, shared, and transmitted values, beliefs, norms, and lifeways of a particular group that guides their thinking, decisions, and actions in patterned ways" (Leininger, 1991, p. 47).

Ethnic Group: "people who identify with, and exhibit, a common heritage...." (Berry, 1990, p. 242).

Ethnic Identity: “a subjective sense of social boundary or a self-definition" (Meleis, Lipson \& Paul, 1992, p. 99).

Immigrant: Any person who moves to a different country legally or illegally with the intent of remaining in that country. A person may move for economic, social, or personal reasons (Heisler, 1992), or from fear of personal harm.

Maternal sensitivity: The "mother's ability to perceive and to interpret accurately the signals and communications implicit in her infant's behavior, and given this understanding, to respond to them appropriately and promptly" (Ainsworth, et al., 1974, p. 127).

Post-traumatic Stress Disorder: The American Psychiatric Association's 1994 definition is exposure "to a traumatic event" of extreme threat to their own or another's physical integrity and responds with "intense fear, helplessness, or horror. . . The event is reexperienced ... with persistent avoidance of stimuli associated with the trauma and numbing of general responsiveness,... lasting over one month,... and the level of functioning is impaired" (cited in Marsella, et al., 1996, pp 31-32).

Post-traumatic stress: "A diverse myriad of stress-related physical complaints and disorders in addition to or in concomitance with ... psychiatric and behavioral disorders". These can be manifested as depression, anxiety, or somatization (Everly, 1995, p. 7).

Refugee: The official United Nations definition is: "A person who is outside the country of her/his nationality (or habitual residence) and unable or unwilling to return to it or to its protection because of actual or threatened persecution on account of race, religion, nationality, membership in a particular social group, or political opinion" (Muecke, 1992, p. 521). This designation is granted to groups or individuals by governments. 
Transition: "movement or passage from one position, state, stage, subject, concept, etc., to another." (Stein \& Urdang, 1967, p. 1505) 


\section{CHAPTER 2}

\section{Review of the Literature}

The purpose of chapter two is to review and critique the literature about maternal sensitivity in mothers who are negotiating the overlapping transitions of migration and becoming parents. It begins by examining the conceptual framework of transition, and emphasizing those overlapping transitions. Next there is a brief discussion of Belsky's (1984) three determinants of effective parenting; personal characteristics, characteristics of the infant, and contextual characteristics. An expanded discussion of contextual characteristics has been reframed to reflect the experiences of immigrant, and specifically, refugee populations. This part of the review is based on the work of Foss (1996). An abbreviated history of the Vietnamese and Hmong follows. Finally, a critique of the research on maternal sensitivity, posttraumatic stress, and acculturation is presented.

\section{Conceptual Framework}

\section{Transition}

Both transition theory (Bridges, 1980) and Belsky's (1984) determinants of parenting provided the conceptual framework for this study. Transition was defined by Chick and Meleis (1986) as a "passage from one life phase, condition, or status to another. .. embracing the elements of process, time span, and perception" (p.239). Bridges (1980) listed three universal stages of transition as endings, the neutral zone and beginnings. He considered an event, or an internal sense of impending change as antecedents for transition. Ending or letting go of a familiar state signals the beginning of the transition process. During the ending stage, there is separation from selected relationships, loss of certain aspects of personal identity, a shedding of selected roles with a loss of associated abilities 
varies, depending on the expected outcome (Bridges, 1980).

For Bridges (1980), the second stage or "neutral zone" is the time when one exists in a suspended state while the new is formed internally. A sense of chaos and uncertainty about the future characterizes that state. One often becomes withdrawn and reflective and begins to explore possible new realities in roles, behaviors, and relationships. The response to this chaos varies with individuals. The final stage is a new beginning with a corresponding "inner realignment and renewal of energy" (p. 136). Here one accepts and creates a new reality. An altered personal identity is forged, different roles acquired, and either new relationships formed or old ones reconfigured. Individual responses depend on internal and external conditions surrounding the process of transition, such as personal meaning of the change and the personal, social and material resources available during transition (Schumacher \& Meleis, 1994). The outcome of transition can range from personal stress to personal growth, measured by a sense of well-being, mastery of new roles, the status of personal relationships, or illnesses (Chick\& Meleis, 1986).

Four types of transition were described by Schumacher \& Meleis (1994). They were developmental, situational, health-illness and organizational. Transition to parenthood, when the arrival of a new infant thrusts parents into altered roles and relationships, typifies developmental transition. Migration, a time when individuals and/or groups move from one society to another, typifies situational transition. When more than one type of transition occurs simultaneously, there is an increased complexity of the process of transition with heightened stress (Chick \& Meleis, 1986). When immigrants become parents, the overlapping of situational and developmental transitions occurs. Although it would be expected that increased stress levels would occur, that phenomenon has not been reported.

Figure 3 illustrates the three stages of transition in simultaneous transitions to parenthood and in transitions to a new culture. Both the loss of a homeland and the loss of personal identity represent the ending stage of transition. The neutral zone is represented by the travel and culture shock stage of migration and by waiting for the birth of the child. A new beginning occurs when immigrants relocate permanently and try to make sense of their new environment. When a baby is born, life as a parent either begins, if this is the first 
Figure 3

Transition and Parenting in Immigrant Populations

\section{Parenthood}
Loss of Identity

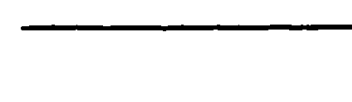

ENDING

Immigration

Loss of

Homeland

$\underset{\text { Wirth }}{\text { Waiting for }}$
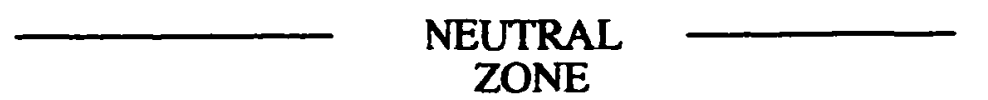

Travel \& Culture Shock

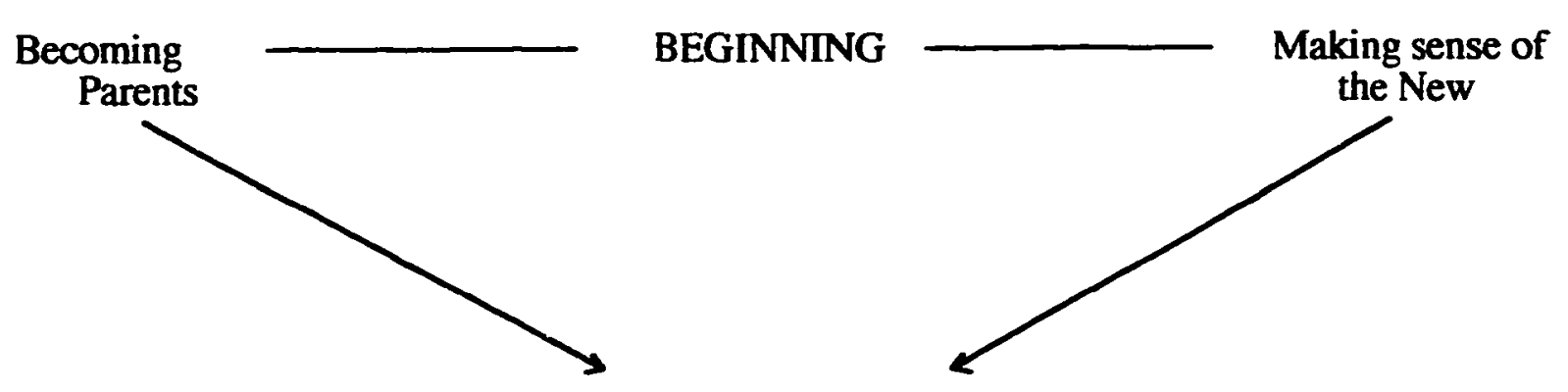

PARENTING IN IMMIGRANT POPULATIONS

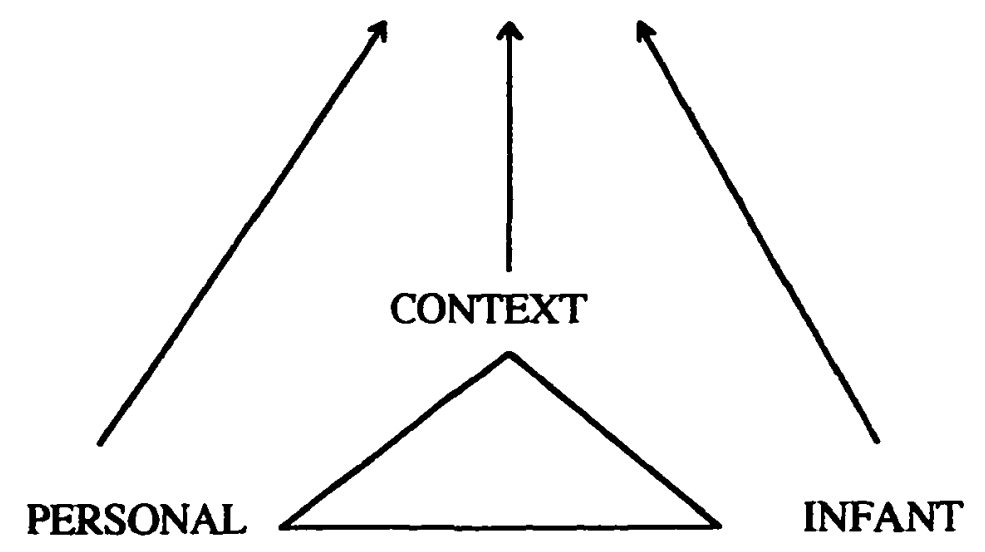


child, or changes to incorporate another child. These overlapping transitions are shown to influence parenting as it occurs in immigrant populations. Belsky's (1984) three determinants of parenting are also shown to determine the quality of parenting in immigrant populations. When the context assumes characteristics reflecting experiences of immigrants (Figure 1), the significance of the transition as part of the context becomes apparent. It is at the intersection of these two theories that parenting in immigrant populations is found. A more detailed discussion of both transition to parenthood and transition subsequent to migration follows.

\section{Transition to Parenthood}

Antecedents of transition to parenthood include the prior mental health status of the mother, her social network, her prior experiences, and expectations about the rewards and demands of children (Mebert, 1991). When subsequent children join the family, part of the transition to parenthood includes changes in the whole family system. Research about transition to parenthood has tended to focus on the marital relationship (Tomlinson, 1996) or on available social support for the mother (Goldstein, Diener,\& Mangelsdorf, 1996; Gottlieb \& Pancer, 1988). That body of research has been conducted primarily among ethnic groups of European descent who configure their families around a nuclear family model (Goldberg \& Michaels, 1988). Recently, the development of a parental role within the context of a family system has received attention in an attempt to understand transition to parenthood in other cultural groups (Stamp, 1994).

As noted in Figure 3, transition to parenthood was divided into the three universal segments described by Bridges (1980). Life as a childless woman or childless couple ends with loss of that associated identity and role (Gottlieb \& Pancer, 1988). For some this change is desired, for bearing children is part of the culturally expected role of women and confers a higher status in the community (Levy-Shiff, 1994; Muret-Wagstaff \& Moore, 1989). For others, anticipation of combining the roles of worker, wife, and mother presents potential for conflict (Levy-Shiff, 1994). Waiting for the birth of the infant represents the neutral zone. During this time the mother experiments with new images. She imagines what the infant will be like. She seeks for information about this new role from family, friends, popular literature, and health care workers (Rubin, 1984). With the birth of 
the infant, a new culturally determined relationship develops with the husband or partner, family, friends, and social networks (Gottlieb \& Pancer, 1988), that generally reflects a "smaller, denser, and more homogeneous network [with] triends who have or are about to have children and family members comprising a large portion of the network" ( $p 259)$. This is the beginning of life as a parent.

Responses to this transition vary, depending on the fulfillment of expectations from the changed relationships and the value and meaning of a mother's infant relative to her particular society and to specific adults within that society (Gottlieb \& Pancer, 1988; Stamp, 1994). At the end of the transition period, successful adaptation to parenthood is expected characterized by three things, a "synchronous parent-infant interaction, development of secure attachment, [and] sensitivity to [the] child's developmental needs" (Field, 1995, p. 356). Unsuccessful completion of this transition often results in unresolved family conflicts or family violence (Stamp, 1994). In addition, it has been shown to be a contributor to maternal depression. Other times, unsuccessful completion of the transition to parenthood can be a result of maternal depression (Campbell, Cohn, Flanagan, \& Meyers, 1992; Fedele, Golding, Grossman, \& Pollack, 1998; Hock, Schirtzinger, Lutz, \& Widaman, 1995).

\section{Migration}

Migration refers to the process of permanently relocating from one geographic location to another. Antecedents for migration are varied. Some immigrants decide to move for economic, social, or personal reasons. Refugees usually move out of fear of harm to self or one's family (Heisler, 1992, Muecke, 1992). This discussion focuses on refugees.

Ending is a particularly apt word to describe the onset of this process, for nearly all that was familiar is lost. Refugees often have experienced permanent separation from their family due to violent social upheavals. Many family members may have been killed, or died from illness, hunger, or accidents. There may have been loss of an accustomed occupation and socioeconomic status, loss of familiar roles within family and society, loss of one's own language as the primary language, loss of familiar social customs, and loss of commonly understood ways of being healthy or ill, as well as how to interact with health care resources (Foss, 1996). Responses to such losses among refugees have been cited as 
the reason for prevailing incidence of depression, anxiety, and post-traumatic stress disorder (Chung, Kagawa-Singer; Nicholson, 1997; Tran, 1993).

The neutral zone can last for an undetermined length of time. Uncertainty about the future and an ongoing sense of chaos begin before leaving and continue until resettlement in a new country or society. Even after relocation, a sense of living in a suspended state continues. The task of exploring new roles, a new language, and often a new occupation with a diminished social status continues. At the same time, a new social or kinship network that was begun in transit, develops (Fox, 1991; Muecke, 1992; Williams, 1990). The health care system in the United States or other highly developed societies frequently introduces unfamiliar meanings for symptoms as well as new ways of interacting with health care providers (Gonsalves, 1992; Meleis, 1991). All of these factors contribute to a varied response to transition, ranging from hope or euphoria, to disorientation, sadness, anger, or guilt (Frye \& D'Avanzo, 1994; Gonsalves, 1992; Williams \& Berry, 1991).

During the last stage of transition, labeled the beginning stage, the immigrant begins to engage with the new environment to create a new reality (Berry, 1990; Gonsalves, 1992). Families and clans have been reported to be the main source of support during this beginning (Thao, 1982; Lipson, 1992; Rumbaut, 1994; Weinstein-Shr \& Henkin, 1991), but they can also introduce added stress (Aroian, Spitzer, \& Bell, 1996). Reorganization of family and clan boundaries frequently occurs with development of new kinship bonds and roles (Thao, 1982; Meleis, 1991; Weinstein-Shr \& Henkin, 1991). Furthermore, new experiences with unfamiliar health care systems and resources have been reported to increase the level of stress of the refugee (Frye \& D'Avanzo, 1994; Juthani, 1992; Meleis, 1991; Rogler, 1994).

The outcome of transition from migration varies. Newcomers develop language, employment, and coping skills on a continuum that allows them to function in the new society at differing levels of personal growth or personal stress (Hauff \& Vaglum, 1995; Rumbaut, 1994; Tran, 1993). One of the most powerful predictors of the ease of the transition in a new country has been related to the individual's capabilities in the language of the new country and the ability to obtain employment (Aroian, 1990). Many, however, have remained isolated due to language barriers, lack of education, financial barriers, and 
perceived discrimination from surrounding residents. Such negative experiences contribute to increased personal stress (Aroian, 1990; Frye \& D’Avanzo, 1994; Yee, 1992).

\section{Determinants of Parenting}

As stated earlier, Belsky (1984) and others (Belsky \& Isabella, 1988) identified three determinants of parenting of infants and young children: (a) parental personality and psychological well-being, (b) child characteristics, and (c) contextual subsystems of support. Belsky considered the parent's contribution to be the most important component of effective parenting. Infant characteristics, either difficult or easy temperaments, played a secondary role. Stress, socioeconomic status, and social support have been the main contextual determinants discussed. For Belsky, stress occurs when there is no support for the mother from either the marital relationship, or the social network, and when the demands of employment decrease the energy available to sensitively interact with her infants and children (Belsky, 1984; Belsky \& Isabella, 1988). A brief summary of pertinent literature about the relative contributions of the mother, infant, and context to effective parenting follows.

Personal

Belsky (1984) stated that anything that interferes with the ability of the parent to notice and respond to subtle differences in infant cues has a negative effect on infant wellbeing. Maternal depression, matemal competence and ethnic identity have all been cited as contributors to personal characteristics and are discussed briefly in this section, and in greater depth in the discussion of maternal sensitivity.

Chronic maternal depression has been extensively documented to interfere with the mother's ability to respond sensitively to her infant. This impedes the development of a maternal-infant relationship (Campbell, et al., 1995; Radke-Yarrow, 1991). Negative life events such as financial impoverishment, social isolation, adverse life events, and a lack of expected spousal or family support have been increasingly identified as predictors of maternal depression in many countries (Aderibigbe, Gureje, \& Omigbodun, 1993; Hobfoll, Ritter, Lavin, Hulsizer, \& Cameron, 1995; Pianta \& Egeland, 1994). Family members and spouses have universally provided the overwhelming amount of support for a new mother (Lantican \& Corona, 1992; Levitt, Coffman, Guacci-Franco, \& Loveless, 1993); however, 
it has been the fit between support received and the support expected by the mother which has been found to predict maternal depression (Koniak-Griffin, 1993; Levitt, et al., 1993; Logsdon, McBride, \& Birkimer, 1994).

An awareness of personal competence as a parent continues to be identified as critical to effective parenting (MacPhee, et al., 1996). Self esteem and confidence in one's ability to acquire a new identity, thus fulfilling the maternal role, have been identified as predictors of the development of maternal competence (Isabella, 1994; Rubin, 1984). MacPhee, et al. (1996) found that a constant predictor of self esteem across a variety of ethnic groups is whether or not the mother feels positively regarded by those she considers important to her. In addition, the culturally determined meaning of the maternal role has been shown to influence the development of maternal identity and maternal competence (Aderibigbe, et al., 1993; Koniak-Griffin, 1993).

Ethnic identity, defined as "a subjective sense of social boundary or a selfdefinition" (Meleis, et al., 1992, p. 99), is a personal parental characteristic that has been increasingly recognized as critical to the development of a maternal role in a particular society (Kim and Rew, 1994). Harkness and Super's (1996) edited book titled 'Parents' Cultural Belief Systems" focused on how parental belief systems in different ethnic groups greatly influences how an infant is reared. In that book, there is an implicit assumption that belief systems are closely linked to ethnic identity. For people making a transition between two cultures, an ethnic identity may reflect both cultures.

Infant

Infants exert force on the mother-infant relationship by virtue of their temperament (difficult or easy), or by their fulfillment of parental expectations (Seideman \& Kleine, 1995). van den Boom and Hoeksma (1994) described difficult infants as having developmental delays or not readily responding to attempts to nurture or comfort and Belsky (1984) rated difficult infants harder to parent. Mothers of preterm infants required more time to acquire a maternal role and identity, and even longer to feel competent using maternal behaviors (Zabielski, 1994). Mothers of difficult infants have been reported to focus on responding to the infant's distress rather than making positive interactions (van den Boom \& Hoeksma, 1994). When examining interactive behavior between mothers and 
infants, contextual variables would be expected to exert an influence on the infant as well as on the mother and family, but such influences were not discussed in the literature reviewed for this study.

The international health care literature described how family care discriminated in favor of males over females (Ardayfio-Schandorf, 1993; Becktell, 1994). No references were located that addressed how such preferences influenced the development of maternal behaviors after migrating to the United States. Studies about whether or not an infant holds a different meaning after relocation to a new society were not found in the literature that was reviewed. Only the writings about the survivors of the Gerinan Holocaust referred to the meaning attached to subsequent births of children after horrific traumas endured by their parents (Solkoff. 1992). However, there have been many anecdotal reports by mothers from Armenian, Hmong, Laotian, Vietnamese, Afgani and Jewish origins who see the birth of an infant in their adopted country as symbolic of a new beginning in a safe environment (Foss, 1995).

\section{Contextual}

Research about contextual determinants has tended to focus on family or social support, and how the lack of such support contributed to maternal depression and insecure infant attachment (Coffman, Levitt, \& Guacci-Franco, 1995; Isabella, 1994; Logsdon, et al.; Mercer \& Ferketich, 1990). Although the literature about the cultural contexts of parenting has been expanding (Harkness \& Super, 1996; Harwood, et al., 1995), immigration as a contextual influence continues to be absent. This discussion focuses on literature that illustrates migration as a contextual characteristic.

Contextual determinants of parenting in immigrant populations were theorized by Foss (1996) as (a) premigration and migration experiences, (b) post-migration experiences, (c) ethnicity, and (d) the social and economic environment (Table 1). As described by Foss (1996), those four categories reflect the transition process (Bridges, 1980). Premigration and migration experiences correspond to the endings and neutral zone stages. Post migration experiences and ethnicity fall into both the neutral zone and beginning stages. Social and economic variables reflect characteristics of the surrounding new society. 
Table I

Contextual Determinants

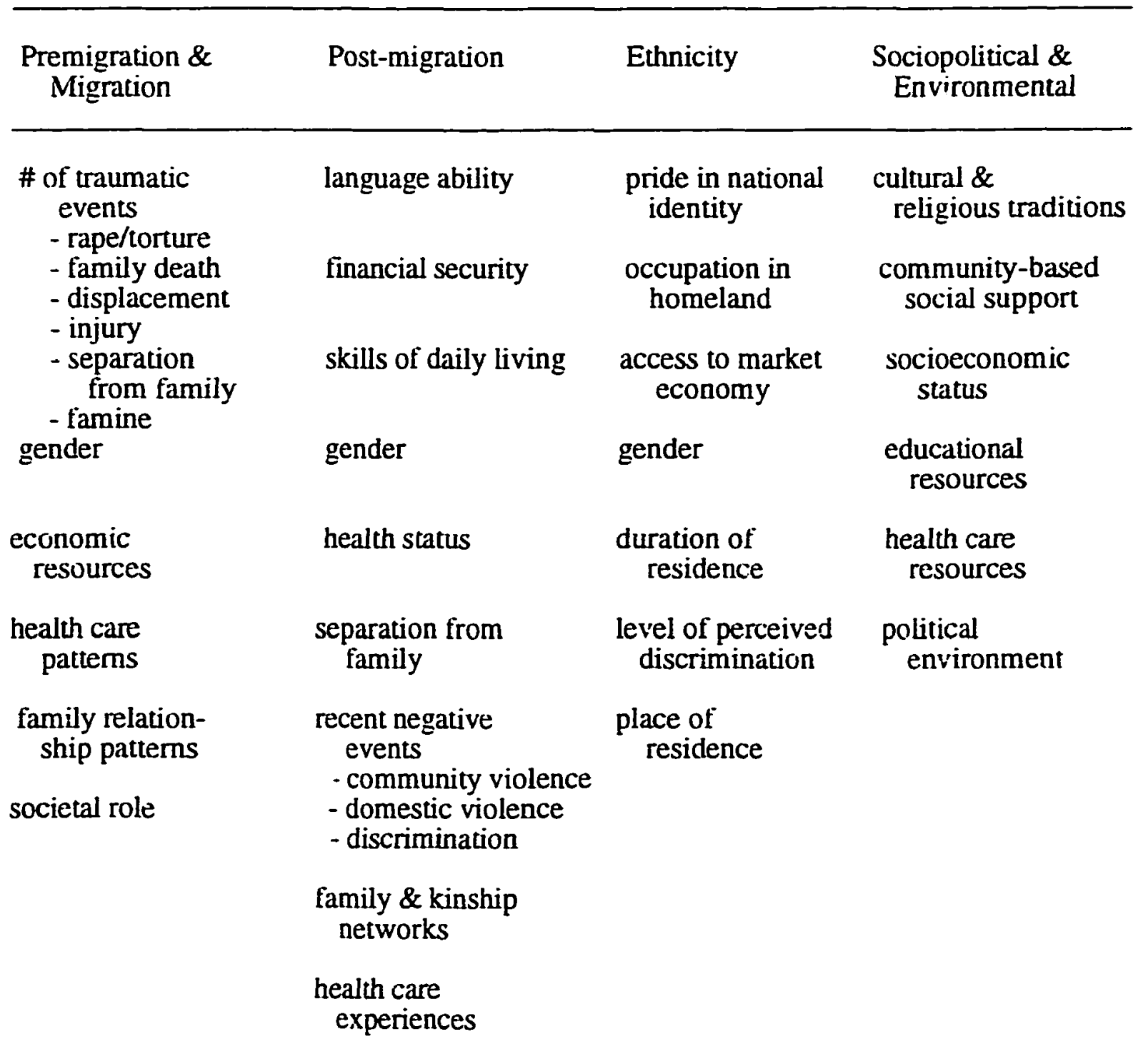

Reprinted with permission from Foss, G. F. (1996). A conceptual model for studying parenting in immigrant populations. Advances in Nursing Science. 19(2), 80. Copyright 1996 Aspen Publishers, Inc.

Specific characteristics are listed for each category of contextual variables that could influence the development of maternal sensitivity in a population of immigrants.

Premigration and migration were the first categories of contextual variables presented by Foss (1996). For refugees, the effects of all the traumas experienced before 
and during migration have been reported to continue long after resettlement (Chung \& Kagawa-Singer, 1993; Hauff \& Vaglum, 1995; Mghir, Freed, Raskin, \& Katon, 1995). Women, in particular, endured losses of family members, familiar social roles and familiar family functions when leaving their homeland (Muecke, 1992; Williams, 199()). Frequently women were raped while fleeing, then later subjected to spousal abuse as a result of their altered status as defiled or dirty women (Friedman, 1992). Because most refugee camps consisted primarily of women and children, female residents were particularly vulnerable to further exploitation or violence (Friedman, 1992; Williams, 1990).

Post-migration was the second category. Major predictors of successful coping after migration include the ability to speak the language, attain financial security, master skills of daily living and maintain physical and mental health (Aroian, 1990; Frye \& D'Avanzo, 1994; Mghir, et al., 1995). Inability to communicate with the surrounding society dramatically decreases social interactions, abilities to become self-supporting and to develop social networks outside the family and kinship system (Aroian, 1990; Meleis, 1991). Chronic family separation after migration has been specifically related to depression and PTSD (Abe, Zane, \& Chun ,1994; Hauff \& Vagluin, 1995).

While the female gender was not specifically identified as a post-migration variable. Foss (1996) reported that this theme implicitly pervaded the literature about refugee experiences in resettlement societies. Domestic violence, and its associated depression and loss of self-esteem, has become a constant part of the post-migration experience for many women (Friedman, 1992; Norton \& Manson, 1992; Phan, 1996). At times, however, positive outcomes may follow refugee camp experiences. The breakdown of traditional and often restricted ways of relating to society, as reportedly occurred in refugee camps (Williams, 1990), has contributed to a sense of empowerment by many women who survived (Fox, 1991; Kibria, 1990). Once in a resettlement society, women often experienced community violence, family violence, decreased social status, and discrimination. When these experiences are combined with the changing roles and functions of various family members, refugee women experience greater degrees of stress (Frye \& D’Avanzo, 1994; Kibria, 1990; Phan, 1996). 
Foss (1996) reported on a growing body of literature about how immigrants develop ethnic identities. Immigrants develop a specific ethnic identity when they earn a living in the new country in the same fashion that they did in their homeland, and develop an assimilated identity when they earn a living as individuals in the manner of the host society (Waters, 1995). She reported that variables influencing the kind and degree of ethnicity claimed by groups included the length of time lived in a host society, the degree of perceived discrimination, geographic location, the professional status of the family when immigrating, and gender. Vietnamese and Hmong groups have developed ethnic communities after relocating in the United States (Hendricks, Downing \& Olney, 1986; Rutledge, 1985). Celebration of festivals such as Vietnamese or Chinese New Year continue and provide ample opportunity to observe how Vietnamese and Hmong conmunities maintain their ethnic identities and traditions.

The last contextual determinant considered by Foss (1996) is the social, political and economic environment. That category includes such things as cultural and religious traditions, educational and health care resources, social support and the sociopolitical environment of the resettlement country. Weinstein-Shr and Henkin (1991) described how community organizations have provided social support to families and have functioned as a bridge between specific groups and government or other community groups. Matsuoka (1993) and Rutledge (1985) related religious adherence and the level of education to the degree of stress reported by Vietnamese in their samples. Lipson (1992) and Meleis (1991) have eloquently described the stress felt by some refugees when health care providers have not understood the ways they express health-related problems nor what refugee women expected from a health care provider.

References to immigration as a specific contextual variable were only found in two articles. Patel, Power, and Bhavngari (1996) investigated how acculturation influences how parents socialize their adolescents to life in the United States. In their study, mothers and fathers combined traditional and new cultures in ways that reflected family goals. Mothers reported different goals than fathers. In France, Stoleru, and Morales-Huet (1991) used two case studies of immigrant mothers to illustrate how mother-infant interaction reflected both North African and French cultural patterns. Their study focused on defining 
contextual variables from both French and North African societies, and illustrating how they influenced the development of mother-infant interactions. Neither study examined how the transitional nature of immigration itself influenced maternal sensitivity.

\section{History}

To better understand maternal sensitivity in Vietnamese and Hmong populations, it is first necessary to understand historical influences on their culture and family. This section briefly reviews the history of Vietnam and Laos. Since both groups share a common Chinese heritage, influences from China are examined. A description of the traditional family structure and the role of women from a Confucian perspective is included. Finally, the Vietnam war and its aftermath are briefly addressed.

\section{Vietnamese}

Ancient records reveal that the Vietnamese people originally lived in the Red River Valley region of what is now North Vietnam. Because of the surrounding harsh conditions. early Vietnamese society developed into closed self-reliant communities in which individual behavior was closely monitored by the whole community. Eventually, invaders from the expansive Han dynasty of China (about 200 B. C. E. - 200 C. E.) subjugated Vietnam for about 1000 years. During this era the elite of both Chinese and Vietnamese societies mingled creating a "new Sino-Vietnamese elite" (Jamieson, 1993, p. 8). Confucianism and Buddhism became well established. Schools followed the Confucian pattern that educated sons from elite families for governmental positions. Women adopted the secondary role assigned to the more educated Chinese women. After 1000 years under Chinese rule, Vietnam regained her independence. For the next 1000 years she was an independent nation, only succumbing to French rule in the late 19th century (Chapuis, 1995; Jamieson, 1993).

Initially, the French sent priests into the southern part of Vietnam to convert the Vietnamese, then to help the French establish centers from which they could gain control of trade centers and eventually, the country. After the French colonized Vietnam, they Romanized the Vietnamese language and established church-sponsored schools making it possible for children to attend for a small fee. Consequently, a large proportion of the 
populace in the South became Christian. As a result of this period of colonization, a broad segment of the country was exposed to French government, language, and food, and the Roman Catholic church (Chapius, 1995; Jamieson, 1993). This made the transition to life in other Westem nations relatively less stressful for refugees from South Vietnam (Gold, 1992).

Confucian philosophy and ethics, introduced by the Chinese, have remained strong influences in the structure of Vietnamese families, their society, and in the relative roles of women and men (Phan, 1996: Rutledge, 1985). Confucian philosophy considers commitment to, and a vigorous pursuit of, moral self-cultivation as the prime obligation (Yu-Wei, 1967). The virtues of shu (reciprocity), jen (benevolence), and li (propriety) remain the cornerstones of Confucianism, and are inherent in observance of filial piety (Bretzke, 1995). Filial piety is a term that refers to the correct manner of interaction between all parties in each of the five central relationships in Confucian thought.

Adherence to the traditional Five Relationships is considered central to a moral life. Those five relationships are paired as follows: (a) ruler and ministers, (b) father and son, (c) husband and wife, (d) elder and younger (brothers), and (e) friend and friend. The three most important pairs are those between king and minister (by extension, all the subjects), father and son, and husband and wife. Confucius taught that when everyone in the community observed the duties and obligations inherent in the Five Relationships, citizens and their communities would be virtuous and moral. Proper observance of these relationships became known as the central part of the virtue of Shu, or reciprocity. Shu is considered to be a Chinese version of the Golden Rule (Bretzke, 1995). Within the context of a moral life, Confucius also taught that submission to the superior person was the key to a harmonious and prosperous society (Hinsch, 1995).

Jen, or benevolence, is a second virtue described by Confucius. It represents the interior progress of an individual in the quest to become a superior person (Bretzke, 1995; Yu-Wei, 1967). Children first learn about benevolence, and thus about filial piety, from learning how to love and respect parents. As they mature, children are expected to extend benevolence and filial piety to the clan and whole society (Yu-Wei, 1987). The virtue of li, or propriety, is an outward expression of jen (benevolence). It is developed by practicing 
constant self-cultivation (Bretzke, 1995; Mei, 1967). This virtue dictates the rules of proper social interactions. To "lose face" means that one has failed in the proper behavior expected in a particular social situation. These three virtues cannot be separated. Tseng Tzu, an ancient Chinese Confucian sage, summarized the inter-relatedness of the three virtues as; "Those who lack propriety in private life, loyalty in serving the sovereign, seriousness in discharging official duties, faithfulness in treating friends, or bravery in waging war are all found wanting in filial piety." (Yu-Wei, 1967).

Before the end of the imperialistic Chinese era in Vietnam, Confucian ethics took on a more religious framework that enshrined the family as the primary community. The most important relationship in filial piety became that of father and son, with the other four relationships assuming supporting roles to this primary obligation. Harmony and virtuous relationships within the family assumed greater and greater importance with the inferior person primarily responsible for maintaining harmony (Kellerher, 1987; Mei, 1967). Thus the son (and all the other children) were responsible for keeping harmony in the relationship with their father and by extension, their mother. It was the wife who was responsible for maintaining harmony in the relationship with her husband and, by exrension, his mother and father, as well as his older brothers (Ebrey, 1991; Kelleher, 1987). These Confucian ideals of moral conduct, harmony and self-improvement have remained a vital part of Vietnamese belief systems to this day, although they are tempered somewhat by a strong Vietnamese tradition of strong women leaders who traditionally have organized their own resources to support their fathers, husbands, and sons in times of crisis (Jamieson, 1993; Phan, 1996; Taylor, 1983).

\section{Hmong}

The Hmong originated in the Transural region of Asia, migrated east to Siberia, then southward into China where many isolated pockets of Hmong people still live. They were always viewed with dislike by the surrounding Chinese because they were fiercely independent, refusing to assimilate into Chinese society as other conquered peoples of China had done. The Hmong never used chopsticks. They ate with spoons. Some Hmong had blonde hair and blue eyes, something the Chinese found reprehensible (Quincy, 1995).

Hmong people vigorously defended their territory and lifestyle from Chinese 
invaders. Over the centuries, many Chinese emperors tried to subdue the Hmong without success. When under attack, the Hmong would retreat to the mountains and conduct guerrilla warfare against the invaders. Many generals with their attacking armies simply disappeared without a trace upon engaging the Hmong in battle. Finally, in 1776, a famous Chinese general nearly wiped out the Hmong warriors and their families (Quincy, 1995). The survivors were separated into five different regions, and forced to wear different colors of cloth to identify them as Hmong groups to the surrounding Chinese (Hamilton-Merritt. 1993; Yang, 1979). They were forbidden to write their own language, but traces of this language remain as symbols and motifs on the traditional "pa dau" embroidery done by Hmong women. Hmong people remain in isolated groups all over mountainous regions of Southeast Asia and China to this day (Quincy, 1995; Yang, 1979).

Chinese, Vietnamese and Hmong people have all shared a belief in the spirit world and in spirit mediums or shamans; however, the Hmong have been more closely identified with the popular religion of China (Quincy, 1995). Popular religion, as well as Taoism, is full of supernatural forces, beings, and magic believed to influence human affairs. Three basic categories of supernatural beings include gods, ghosts, and ancestors, with gods assuming the most importance. Gods usually mirrored the old Chinese bureaucratic hierarchy, but many continue as patrons of various activities of life. Ghosts are souls without male descendants to offer food, money, housing or clothes. They are considered mean and malicious because they are unable to become ancestors. Ancestors, on the other hand, have male children to honor them with gifts and housing (Overmeyer, Arbuckle, Gladney, McRae, Taylor, et al., 1995).

Divination, including shamanism or spirit mediums, ghost writers, and geomancers, are sought when intervention with the spirit world is needed. Intervention could be needed to placate ghosts or angry ancestors, or identify the cause and remedy of an illness (Overmeyer, et. al., 1995). Women have had a more significant role in popular religion and Taoism than they did in Confucianism or Buddhism. They could be shamans, divination experts, or fortune tellers, and were frequently sought by women for childbirth and infancy problems (Reed, 1987). When consulted, a shaman (or spirit medium) enters a trance, travels to the spirit world to identify the cause of the problem, and determine the 
remedy. Upon returning from the trance, he or she instructs the family and the individual how to remedy the situation (Kinsley, 1996). Shamanism, and other aspects of popular religion continue in Hmong societies to a greater extent than in Vietnamese societies. Hmong people in the United States continue to use geomancy (Tapp, 1982), shamanism (Lemoine, 1986), and herbs for healing, (Cheon-Klessig, Camilleri, McElmurry, \& Ohlson, 1988; Thao, 1986).

The Hmong also have maintained a tradition of filial piety. Because the Hmong were always poor (at least as far as is known in recent history), and lived at a subsistence level in China and Laos, they have not been as strict about some aspects of gender relations as have the Vietnamese (Quincy, 1995; Barney, 1979). Donnally's (1994) description of Hmong family relations is identical to that prescribed by traditional Chinese filial piety. She described how the Hmong community in Seattle prides itself on its Hmong history and identity. Part of that identity regards traditional gender relations as something that peculiarly identifies them as Hmong, and they strongly resist changing the nature of those traditionai family relationships and role expectations. Men and women have different spheres of activity and influence and are expected to restrict activities to those specific spheres. Men are the ones who make contracts. Women are not expected to enter into any contractual agreement with another person, family, group, or organization. In her words "ties are strongest between related men, and women think of themselves as the glue that holds these families together" (p. 189). If domestic violence occurs, it is the wife's father and brothers who intervene, for the marriage is seen as a contractual agreement.

\section{The Vietnam War}

The prolonged war in Vietnam and Laos has been well documented in the popular and professional literature. Images of widespread death, destruction, injuries, tears, and desolation quickly come to mind whenever the Vietnam war is mentioned. All Vietnamese and Hmong people who have entered the United States as refugees have done so due to their past associations with French and American military forces (Yang, 1979; Jamieson, 1993). They have brought with them accounts of unspeakable horrors, pain, and suffering as a result of those connections. This section briefly examines that era.

Ever since the advent of French colonialism, alliances were formed by the 
Vietnamese and Laotians with or against the French. What originally began as a quest for freedom from colonialism became a dual between communism and democracy. For both South Vietnamese and Hmong peoples, the withdrawal of the American troops in 1975 signaled the beginning of a purge of the defeated national population. Limited numbers were evacuated from Vietnam and Laos with the Americans in 1975. They were the most educated, wealthy, and the elite of both societies (Gold, 1992; Jamieson, 1993; HamiltonMerritt, 1993). In Vietnam, it was the governmental and military leaders who were evacuated with the Americans (Rutledge, 1985). Many were ethnic Chinese who historically had been viewed with suspicion by indigenous Vietnamese (Jamieson, 1993). In Laos only about a thousand military men who held the highest rank were evacuated in 1975 (Hamilton-Merritt, 1993). Everyone else was left to escape as they were able. Supporters of the United States were fearful of their fate if they stayed in their homeland, so many tried to leave. Their fears were substantiated, for supporters of the United States in both countries experienced execution, labor camps, torture, rape, starvation, loss of home. property, and economic livelihood, and always public scom (Jamieson, 1993: Quincy, 1995). Those who left with the American troops or immediately afterwards became known as the first wave of refugees. People who fled after the end of 1976 were less wealthy or educated, and many were from rural areas. They are considered to be part of the second wave of refugees (Gold, 1992; Haines, 1989).

After 1975, flight from Vietnam by small boat was the most frequent mode of escape. Once away from the shore, however, the horrors did not end. Many boats were small and overcrowded, with the result that many capsized. Many people were washed overboard by large waves and drowned. Others were attacked by pirates, losing all their possessions. When attacked, women and girls were usually raped. If rescued by people friendly to their plight, the survivors were sent to refugee camps, where it was hoped that a sponsor from some nation would agree to grant them asylum (Gold, 1992; Jamieson, 1993; Muecke, 1992). Once in the refugee camp, safety was not assured. Again, many women were raped by camp guards. The camps were overcrowded, and there was insufficient food, clean water, or medical care (Friedman, 1992; Mattson, 1993; Williams, 1990). After the early 1980s, most Vietnamese who immigrated to the United States did so 
under the Orderly Departure Program (ODP). This was a program established by the Vietnamese government to enable Vietnamese citizens to leave Vietnam legally (Gold, 1992; Rumbaut, 1996). Only those who had family in the United States, and had papers stating the United States approved of their entry, could emigrate under that program.

Conditions were much different for the Hmong. As second class citizens in Laos they had not been given the vote, nor they did have schools available to them, unless a mission established one in their community. Hmong men had only been allowed to serve at the lowest ranks of the military. They were excluded from governmental posts and from any meaningful participation in the Laotian common life. Only cne Hmong man eventually participated in the government, and only one Hmong man became a general in the Laotian army. He led remarkably successful battles against the communist Pathet Lao. The majority of the population either raised opium as a trading crop for the Laotian government or sold vegetables from their garden in the market (Quincy, !995).

Because the Hmong had been such successful fighters, at the close of the war they were targeted for extermination by the communist Pathet Lao government. This effort was amazingly successtul. Survivors reported mass killings of men women and children in the most horrible manners imaginable. Some were buried alive, some were disemboweled, and others were progressively amputated. Women were gang raped while forced to watch their children killed and husbands tortured to death. In addition, reports of deadly biological and chemical attacks on Hmong civilians were substantiated (Hamilton-Merrit, 1993). The only route for escape was overland, over mountainous territory and across the Mekong river into Thailand. Once in Thailand the Hmong had to wait years before admission into other countries. Many lived in refugee camps for five, six, seven, or more years before accepted into another country (Hamilton-Merrit). In the camps they lived in extraordinarily crowded conditions and were subjected to further personal violence, rape, or starvation (Friedman, 1992; Hamilton-Merritt, 1993; Quincy, 1995). There has never been an Orderly Departure Program for the Hmong, thus many still experience long waits in refugee camps in Thailand (Quincy, 1995).

With the arrival of Vietnamese and Hmong refugees, social and relief agencies placed them all over the United States with church groups or private individuals who had 
agreed to assist the newcomers adjust to the new society (Rutledge, 1985). As a result, subsequent groups of refugees from Vietnam and Laos found an existing community of people who shared their experiences and ethnic identity and usually provided a supportive ethnic network (Haines, 1989; Hendricks, et al., 1986; Rutledge, 1985). Tensions frequently arose between the first wave early arrivals and newcomers (Gold. 1992).

Eventually many Vietnamese and Hmong chose to relocate to regions where large kinfolk and clan groups had already gathered. Those areas also tended to have a climate more similar to their homelands. Consequently Vietnamese and Hmong populations in Caliromia and Texas exploded (Celano \& Tyler, 1991; Haines, 1989; Rutledge, 1986). Effects of all these experiences on both Vietnamese and Hmong people have been high levels of depression, anxiety and PTSD. How all these experiences have influenced the ability of Vietnamese and Hmong mothers to respond sensitively to their infants was not addressed in any of the literature that was reviewed.

\section{Matemal Sensitivity}

The classic definition of maternal sensitivity comes from Mary Ainsworth, a child development physician who has spent her professional career studying maternal infänt interaction in African and American societies. She defined maternal sensitivity as a "mother's ability to perceive and to interpret accurately the signals and communications implicit in her infant's behavior, and given this understanding, to respond to them appropriately and promptly" (Ainsworth, et al., 1974, p. 127). Seifer, Schiller, Sameroff, Resnick, and Riordan (1996) further refined and described matemal sensitivity as an "alertness to infant signais, appropriate interpretation of response, promptness of response, flexibility of attention and behavior, appropriate level of control, and negotiation of conflicting goals" (p. 13). Maternal sensitivity as a construct has been validated in many different societies (De Wolff \& van IJzendoom, 1997). A recent study in Chile confirmed once again that the construct of maternal sensitivity is neither a culture-bound syndrome, nor found only in particular socioeconomic groups (Valenzuela 1997).

Maternal sensitivity has increasingly come to refer to sensitive behaviors from anyone in a primary care-giving role. "Maternal" as a descriptor frequently has been dropped. Sensitivity has also been used as an umbrella term to refer to characteristics of 
interactive behavior between parent and infant. The specific characteristics of a sensitive parent, however, have remained the same as originally described by Ainsworth in 1973 (van den Boom, 1997). This study focused on the sensitivity of Vietnamese and Hmong mothers to their infants.

Bowlby (1982) and Ainsworth (1967) described the infant's need for a constant care giver to whom that infant could become attached during early life. While it is clear that care givers other than the mother can and do develop significant relationships demonstrating attachment with an infant, the nature of the relationship between the mother and infant continues to be universally recognized as the primary bond during early life (Jackson, 1993; Tronick, Morelli, \& Ivey, 1992; Zimmerman \& McDonald, 1995). From that secure base the infant is free to explore his or her environment and return to the mother for comfort or safety as needed. Infants who do not become securely attached to a primary care giver have been presumed to be at higher risk for subsequent developmental and behavioral problems (Bowlby, 1952; Isabella, 1993; van IJzendoom, Goldberg, Kroonenberg, \& Frenkel, 1992 Valenzuela, 1997).

A recent extensive meta-analysis of antecedents to a secure attachment (De Wolff \& van Uzendoorn, 1997) placed sensitivity as the major characteristic of a parent that predicts securely attached infants. Other characteristics are synchrony, mutuality, a supportive presence, a positive attitude, and stimulation. Additional predictive characteristics of a sensitive mother have been described. Isabella (1994) found that the degree of the mother's satisfaction with her role as "mother" predicted her sensitivity to her infant and thus to a secure infant attachment. Mercer and Ferketich (1990), found that parental competence predicted attachment and that self-esteem was a major predictor of parental competence. Maternal depression has been related to decreased sensitivity (Karl, 1991; Radke-Yarrow, 1991), and negative life events have predicted matemal depression (Aderibigbe, et al., 1993; Pianta \& Egelend, 1994). Self-esteem has been found to mediate between negative life events and maternal depression (Hall, Kotch, Browne, \& Rayens, 1996). If the mother had high self-esteem she was not as likely to become depressed. On the other hand, lower self esteem contributed to depression. None of these studies included refugees or Southeast Asians in their samples. 
If a mother is depressed, she cannot respond with the same degree of sensitivity to her infant. Beck (1996) identified several themes pervading depressed mothers and their interactions with their children. Some mothers "erected a wall to separate themselves emotionally and physically from their children" (p. 101). Many found themselves oversensitive to stimuli, and therefore ignored infant cues. Feelings of guilt and/or uncontrollable anger were often part of their daily experiences. For these mothers, it was not surprising that their infants exhibited less interest in their surroundings and had more expressions of sadness and anger (Pickens \& Field, 1993), did less well on tests of mental development (Murray, 1992) and, as toddlers,experienced more sleep disturbances (Zuckerman, Stevenson, \& Bailey, 1987). Nor is it surprising that depressed mothers report their infants to be more vulnerable (Field, 1995) and rate their infant's behavior more negatively than health professionals (Field. Morrow, \& Adlestein, 1993).

A strong link between maternal depression and negative life events has been articulated by many researchers. For example, Pianta and Egeland (1994) found that symptoms of maternal depression were related to current stressful experiences, including personal or family health problems, family violence, single parenting, and financial difficulties. Murray (1992) found that a lower social class exacerbated the negative results of infant mental development tests. Shaw and Vondra (1993) found that the more closely negative life stressors were related to maternal functioning, the less securely the infant was attached. In a seminal study of mothers living in a very poor area of a city in Chile, Valenzuela (1997) found that well nourished children had more sensitive mothers and were predominantly securely attached to their mothers. In contrast, chronically undernourished children had less sensitive mothers and were primarily anxiously attached. Immigration as an adverse event was not addressed in this body of reviewed literature.

No studies were located that investigated maternal sensitivity in Vietnamese mothers, and only two studies were located that investigated sensitivity in Hmong mothers (Muret-Wagstaff \& Moore, 1989; Oberg, et al., 1986). Between 1982 and 1983 MuretWagstaff and Moore compared maternal sensitivity between Hmong and EuropeanAmerican mothers. They began the study at birth and followed the mothers for one month. They measured infant states and behaviors with the Brazelton Neonatal Behavioral 
Assessment Scale (BNBAS), the mother's behavior with Ainsworth's Sensitivity vs. Insensitivity to the Baby scale, and the environment with the Observation for the Measurement of the Environment Scale (HOME). The sample included 21 pairs of low income Hmong and Caucasian mothers. Actual scores were not given, but Hmong mothers scored consistently close to the ceiling on the Sensitivity Scale, while Caucasian mothers scored considerably lower. The authors attributed the differences between the two cultures to differences about the perceived roles of mothers and the place of infants in family structures. Hmong women valued the role of mother. This was their expected career. The whole community supported them in that task. Caucasian women, on the other hand, did not have a singular role expectation of motherhood nor the extended family support. The authors postulated that the results in the Caucasian population reflected the "susceptibility of urban families to depression, emotional disturbance, marital discord, and family breakdown" (Murat-Wagstaff \& Moore, p. 337).

The second study (Oberg, et al., 1986) compared twelve Hmong and Caucasian mother-infant dyads on parenting skills and four of Ainsworth's global scales: Sensitivity vs. İnsensitivity, Cooperation vs. Interference, Acceptance vs. Rejection, and Accessibility vs. Ignoring and Neglecting. The children in their sample were all twenty-month olds, and had been recruited in a well-child clinic. The results of the clinic-administered measurements were as follows: the mean score for Hmong mothers was 8.58 on a ninepoint scale, while the mean score for Caucasian mothers was 5.17. Again, the authors attributed most of the differences to family support for the mother and infant. Infant health outcomes were examined as a way to validate that significant differences did occur between the mothers. Hmong infants had a history of fewer accidents at home, fewer emergency room visits for accidents, and their nutritional level was superior.

Another major concern reported in the literature is how to actually measure maternal sensitivity in a culturally appropriate manner (Goldsmith \& Alansky, 1987; Seifer, et al., 1996; Strayer, Verissimo, Vaughn, \& Howes, 1995). Influences from the culture of the parent when evaluating interactive behaviors must be considered. Chapter 3 addresses this issue. 


\section{Posttraumatic Stress}

As stated earlier, Posttraumatic Stress Disorder (PTSD) was defined in 1994 by the American Psychiatric Association (cited in Marsella, et al., 1996) as a response to exposure "to a traumatic event of extreme threat to their own or another's physical integrity ... [with a response of] ... intense fear, helplessness, or horror" (pp. 31-32). Functioning is impaired because the event is reexperienced, stimuli associated with the trauma are avoided, and affective responses are diminished to the point of emotional numbness. To meet the criteria for PTSD, symptoms must continue for at least one month. Postraumatic stress has been defined more broadly as a "diverse myriad of stress-related physical complaints and disorders in addition to or in concomitance with ... psychiatric and behavioral disorders" that can be manifested as depression, anxiety, or somatization (Every, G. S., 1995, p. 7). Many without a diagnosis of PTSD suffer from postraumatic stress (PTS) as a result of past traumas or negative life events.

Most of the research on PTSD was done by psychiatrists who were evaiuating a medical diagnosis in clinical samples of veterans (Everly \& Lating, 1995). More recently research focused on women and children who were abused. Finally, research about PTSD and PTS in refugees has been reported (Marsclla, et al., 1996). PTSD has been highly correlated with depression, anxiety and somatization, thus most studies have used measures of those problems, specifically depression and anxiety, when assessing PTSD in clinical samples of Southeast Asians (Abe, et al., 1994; Chung \& Kagawa-Singer, 1993; Hauff \& Vaglum, 1995; Kroll, Habenicht, Mackenzie, Yang, Chan, et al., 1989; Mollica, Wyshak, Lavelle. Truong, Tor, \& Yang, 1990; Westermeyer, 1995). At times, only measurements of depression and anxiety have been used to reflect the presence or absence of posttraumatic stress (Mollica, et al.,1987; Mouanoutoua \& Brown, 1995). Although the focus of this study was on PTS rather than PTSD, since the difference between the two terms is a matter of degree and much of the literature does not distinguish between PTSD and PTS, this review includes some of the literature about PTSD.

The most common affective problems reported among Southeast Asian refugees have been depression, anxiety, and somatization (Beiser, et al., 1989; Mollica, Wyshak, \& Lavelle, 1987; Nicassio, 1985). Many studies found $25 \%$ to $50 \%$ of sampled Southeast 
Asian participants in general clinics experienced depression or other affective disorders (Chung \& Kagawa-Singer, 1993; Kroll, et al., 1989: Mollica, Wyshak \& Lavelle, 1987: Westermeyer, 1988). In a non-clinical study, Hauff and Vaglum (1995) found that $38.5 \%$ of the women and $18.3 \%$ of the men experienced high levels of psychological distress. usually depression (Hauff \& Vaglum, 1995), and Rousseau, Drapeau, and Carin, (1997) found that $37.6 \%$ of the parents of Southeast Asian children in a public school had a clinical level of depression.

Research has shown that reporting physical symptoms instead of psychic pain is common after experiencing traumatic events (Mathews, Scheier, Brunson, \& Carducci, 1989; Waitzkin \& Magana, 1997). In addition, when PTS, depression, and anxiety have been found in cultures that identify strongly with China, physical complaints are commonly used to express psychic distress (Hong, Lee, \& Larenzo, 1995; Zhang, 1995). As described earlier, Confucian philosophy emphasizes maintenance of harmony, balance, and proper behavior. Expression of emotions is not considered proper hehavior: in fact, it is considered uncouth (Zhang, 1995). The most commonly reported physical symptoms include poor appetite, headaches, lack of energy, shortness of breath and sleep problems (Hong, et al., 1995). It is possible that rates of depression, anxiety, and PTSD among the Vietnamese and Hmong have been underreported unless care was taken to ensure that physical complaints were included in the assessment of psychological status.

Recent negative life events appear to contribute as much as premigration traumas to the incidence of depression and anxiety. Hauff and Vaglum (1995) found that recent negative life events with chronic family separation were two of four significant predictors of PTS in his sample of Vietnamese people. Abe, et al., (1994) found that stressful life events subsequent to resettlement in the United States differentiated between Southeast Asian refugees with and without PTSD. The two discriminating variables were the degree of anger about the experiences that was reported and continuing dependence on public assistance. Chung and Kagawa-Singer (1993) also reported that the longer participants had lived in the United States, the greater their level of depression and anxiety. Again, women reported more depression and anxiety than men.

Nicholson's recent investigation (1997) into premigration and post-migration 
stressors on mental health of Southeast Asians supported Foss's (1996) conceptual framework. Nicholson examined a gender and ethnic balanced community-based sample of 447 Southeast Asians living in the Boston, MA region. There were 110 participants from each major ethnic group; Vietnamese, Cambodian, Laotian, and Hmong. All had immigrated at least four years prior to the study. She used the Hopkins Symptom Checklist-25 (HSCL-25) to measure PTSD, depression, and anxiety, and the Harvard Trauma Questionnaire (HTQ) to measure PTSD, and relate symptoms to premigration or post-migration traumas. Both tools include questions about physical symptoms that may indicate psychic distress.

A path analysis was then constructed showing the relative contributions of premigration and post-migration negative life events to the outcomes of PTSD, anxiety, and depression. Fourteen percent of her sample met criteria for PTSD, 40\% had clinical depression, and 35\% had clinical anxiety. The percent of each category who were women was not reported. Two post-migration factors, the degree of current stress and the perceived health status, were the two major direct predictors of the outcomes PTSD. depression, and anxiety. The third post-migration factor, income, only predicted depression. Premigration and migration factors predicted mental health outcomes both directly and indirectly. The number of traumatic events experienced or witnessed betore and during migration predicted directly the degree of PTSD, but only the actual number of events that were personally experienced predicted all three post-migration factors. The female gender predicted anxiety and income directly, and thus predicted depression indirectly, while being unmarried predicted depression directly.

Many researchers confirmed that women experienced higher levels of depression and anxiety than men (Chung \& Kagawa-Singer, 1993; Hauff and Vaglam, 1995; Kroll, et al., 1989; Nicholson, 1997; Tran, 1993). Many reasons have already been cited in this review of the literature. They are briefly summarized here. In addition to experiencirig personal violence during transit, many women lost husbands and other close family members before and during the escape from Vietnam and Laos (Mattson, 1993). Without an extended family, there is less support and less income (Carlin, 1990). Many women were sexually assaulted before and during migration. Some were then further exposed to 
family violence (Friedman, 1992; Frye \& D'Avanzo, 1994; Williamı, 1990).

Studies in the general population have shown higher levels of depression, anxiety, and PTSD in women who have been exposed to sexual assault and family violence (Root, 1996). No studies reporting the incidence of family violence and sexual assault in the Southeast Asian community were located, but given the history of their escapes, anecdotal reports, and scattered case studies, a relationship could be expected (Phan, 1996).

Tran (1993) found that self-efficacy, or a sense of control over one's existence, predicted depression. Situations that seem out of personal control or do not have meaning within the surrounding capabilities have been related to increased levels of anxiety and depression. Depression has been related to a lower level of education and limited ability to speak the local language. That combination isolates many women. If there are young children, women are even more isolated at home. Obtaining medical care remains a major problem when transportation, language, and literacy are barriers (Aroian, 1990; Carlin, 1990; Mattson, 1993). In addition, increased anxiety levels vere reported by Liebkind (1996) when experiences of prejudice and discrimination were reported.

In 1992, Muecke recommended that instead of focusing exclusively on the problems and subsequent ill health experienced by Southeast Asians since the Vietnain War, researchers explore possible protective factors operating in that population. Given the different levels of PTS, depression, and anxiety found in the four major groups of refugees from Southeast Asia, some have recently begun to offer an explanation for the varying levels of distress.

Zika and Chamberlain (1992) found a strong correlation between meaning in life and psychological health. Meaning is closely related to a sense of coherence. The presence or absence of a sense of coherence (Antonovsky, 1987) has been proposed as an explanation for differing levels of distress between the Vietnamese and Hmong (Yin \& Akutsu, 1997; Yin, Akutsu, Zhang, \& Huang, 1997). A sense of coherence is (a) an awareness that life's events are predictable and comprehensible, (b) one has available resources to manage life's current and future experiences, and (c) life experiences provide meaning within the person's world view. The essence of a sense of coherence is that there are things in life the individual cares about and considers "worthy of commitment and 
engagement" (Antonovsky, 1987, p 18). Therefore, having a sense of coherence would be expected to predict better health. Feeling demoralized, or that life made no sense, would be expected to predict poorer health, i.e., depression and anxiety.

Yin and Akutsu (1997) found that a sense of coherence did predict happiness, and thus well-being. Consistent with Antonovsky 's framework, they used the terms happiness and well-being to refer to positive health, and the term demoralization to refer to such things as depression and anxiety. In their sample, Vietnamese participants had the highest sense of coherence among all four major Southeast Asian groups, and correspondingly, the greatest feeling of happiness. The Hmong, on the other hand, were the most traditional and reported the lowest sense of coherence with correspondingly low feelings of happiness. Yin, et al., (1997) found that in addition to experiencing substantial anxiety, the Hmong were the most depressed of all the Southeast Asian groups. Part of their explanation was that the Hmong were the most culturally traditional whereas the Vietnamese were the least culturally traditional. Cultural traditionalism was defined as "an inflexible clinging to the culture of origin and refusal to explore the new mainstream cultural context" (p.856). Hmong men exhibited more cultural traditionalism than women. Similar findings about cultural traditionalism among Hmong men were also reported by Donnally (1994).

The role of resilience, or the ability to function adequately in the face of major life losses in refugee health has been cited as another possible reason for positive health in refugee groups (Muecke, 1992). In a comprehensive review, Jacelon (1997) identified both a trait and process of resilience. The trait of resilience was described as a personal characteristic that developed over time by continued exposures to stressful situations. The process of resilience entailed two steps; first, attempting to minimize the effects of the stressor and responses to it, and secondly, accepting the new reality and reorganizing life around it.

Many of the post-migration traumas discussed in the literature were related to acculturation. The term acculturative stress refers to stress experienced as a result of the acculturation process. Prior to exploring acculturative stress among Vietnamese and Hmong peoples, the concept of acculturation is briefly examined. 


\section{Acculturation}

Acculturation is a term often used in the health care literature to reflect the process of entering a new society, then changing personal identity and behaviors to more closely conform to the dominant group. Current thinking acknowledges that changes occur on both sides of the acculturation process (Berry, 1990; Bun \& Kiong,1993; Heisler, 1992), however acculturation has usually been described from the dominant group's perspective (Nicassio, 1985). A standard definition of acculturation is "culture change that is initiated by the conjunction of two or more autonomous cultural systems. . . [and] results in population-level changes that are due to contact with other cultures" (Social Science Research Council, 1954, cited by Berry, 1990, p. 233-234). It occurs at both the individual and the group level.

The transitional nature of migration has been described as part of the conceptual framework. As with all transitions, stress comes from the actual move and subsequent attempts to make sense of the new environment (Aroian, et al., 1996; Baptiste 1993: Berry, 1986; Gonsolves, 1992). This section first summarizes the nature of acculturation among Vietnamese and Hmong, then discusses acculturative stress. Acculturation of Vietnamese and Hmong Peoples

The society found in the United States reflects strong independent individualistic values rather than a society that revolves around family or clan needs and family connections (Finck, 1982; Gold, 1992; Rutledge, 1985; Thao, 1982). In spite of moving to an individualistic society, both the Vietnamese and Hmong have maintained a strong family and clan focus since arriving (Gold, 1992; Hendricks, et al., 1986). Many have relocated to be near family members (Celano \& Tyler, 1991; Haines, 1989; Rutledge, 1986). Coming from a Confucian value system that stresses filial piety, connecting with remaining family and clan members not only eases the impact of multiple changes, but also provides a community with whom to celebrate festivals that honor ancestors.

Vietnamese women have been reported to support maintaining the traditional Confucian-based family relationship that existed in Vietnam (Kibria, 1990, 1993). They were in favor of a traditional family structure that upheld a husband's responsibility to 
support the family economically, and the children's responsibility to provide economic support for their parents as they became able to do so. In Vietnam, women had built on traditionally strong relationships with other women to achieve their goals for themselves and their family. Community gossip frequently was used to exert pressure on a husband to make a specific choice in order to save face or maintain propriety. As a result, Phan (1996) questioned the extent of domestic violence among the Vietnamese due to the general disapproval of physical violence toward wives and children that exists in the Vietnamese culture. If domestic violence did occur, older female family members intervened to stop it.

Vietnamese women have reported more difficulty adjusting to different role expectations in the United States than men (Matsuoka, 1993). Kibria (1993) expanded on the same theme. Women have had to interact with many community agencies such as utilities, schools, and health care providers in a new country with a different language and expectations. In Vietnam, the men were the ones who interacted with the bureaucratic institutions. The economic needs of the tamily in the United States require that women work outside the home in addition to maintaining responsibility for the organization and functioning of the family. Responsibilities have been added, while none have been removed.

Other value differences between Vietnamese refugees and more established citizens were illustrated by a comparison of values between a sample of Vietnamese and Amcricans (Penner and Anh, 1977). In that study, both Vietnamese and Americans ranked world peace, family security, happiness, and a comfortable life among the five most highly valued goals. The ways participants thought those goals should be achieved, however, demonstrated major differences. Vietnamese men ranked honesty, obedience, forgiveness, independence, and courage as the top five contributors to attainment of their goals. Vietnamese women ranked obedience first, even over honesty. Americans, on the other hand, ranked (in order) honesty, responsibility, ambitiousness, forgiveness, and broadmindedness as the top five values contributing to the attainment of their goals. Obedience was sixteenth in their list of values that contributed to attainment of goals. The authors attributed the Vietnamese responses to a pervading Confucian philosophy and ethic in the Vietnamese society. They attributed the women's higher ranking of obedience to their 
acceptance of filial piety as part of their Confucian ethical system. Although no such comparison of specific values has been located in the Hmong community, given the common Chinese heritage of both groups one might expect similar findings.

Donnelly (1994) reported that the individualistic American society was bringing changes to the Hmong. She stated that many Hmong were embracing a fundamentalist Christianity that endorsed individual personal responsibility and more gender equality than traditionally was expected. The explanation offered was that they are in a different society with different needs and healing systems where their traditional beliefs are not as relevant as they were in their homeland (Capps, 1994; Scott, 1986). Adler (1995), however, attributed the incidence of Sudden Unexpected Noctumal Death Syndrome (SUNDS) among Hmong men to a conflict between traditional spirit beliefs and Christian beliefs. Hmong men are the ones expected to honor their ancestors by offering them food and a place to live. If neglected, the ancestor will leave the household, making the living memibers, especially the male head of household, vulnerable to evil spirits who will steal his soul, thus causing his death.

Younger Hmong have begun to pattern their living arrangements more as a nuclear family rather than living as members of a closely knit clan. The result of these shifts has been that women are beginning to exert more power within the family system. In one reported instance of domestic violence, the wife even sought divorce (Donnelly, 1994). In the case she described, the brothers supported that action because they did not like or respect the husband. This is in contrast with the traditional method of dealing with domestic violence that reflects filial piety. Usually the victim's father and brothers seek clan judgments against the offender, and restitution is made to the victim's family for violation of its honor by paying sums of money to the father or responsible brother. Women are not expected to articulate personal needs nor participate in the resolution (Goldstein, 1986).

\section{Acculturation and Health Status}

While the focus of most research about acculturation in the refugee population has been on acculturative stress and ways to treat PTSD, anxiety, and depression, other healthrelated associations with acculturation have been found. Scribner and Dwyer (1989) were the first to relate increased acculturation to lower birth weights. They examined more than 
1,600 responses from mothers of Mexican descent, collected as part of the Hispanic Health and Nutrition Examination Survey (HHANES), for possible predictors of low birth weight. Smoking was predominantly found in mothers born in the United States, and was also found to be a major predictor of low birth weight. The reason for smoking was attributed to increased acculturation of the mother. Cobas, Balcazar, and Benin, et al. (1996) performed a structural equation model on the same data and found that speaking English had the strongest relationship to both smoking and having a low birth weight baby. In another study, using the same data base, Guendelman, Gould, Hudes, et al., (1990) found that Hispanic mothers born in the United States had a $60 \%$ higher risk for having a low birth weight baby than did mothers born in Mexico. They also related this much higher rate to smoking, alcohol, and drug use. In addition, Zambrana, Scrimshaw, Collins, et al. (1997) reported that the more a Mexican mother felt integrated into the surrounding society, the more stress she felt from the lack of social support from her family, and from negative life events. Stress, in turn, was related to increased substance use, low birth weight and to early delivery.

A different study using data from the large National Linked Birth and Infant Death data sets also found that women born in the United States had much higher rates of low birth weight babies and infant deaths than women from all ethnic groups who were bom in another country (Singh \& Yu, 1996). This study compared several major ethnic groups, including Vietnamese and Hmong. Unfortunately, Vietnamese and Hmong were included in an Other Asian category, thus it was not possible to identify specific trends for them. When foreign-born mothers from the Other Asian category were compared to Americanbom mothers, there was an $11 \%$ difference in low birth weight between the groups. Smoking rates for American-bom mothers in the Other Asian group was 11\%. For foreignborn mothers it was only $3 \%$.

Two studies of the relationship of low birth weight and acculturation among Southeast Asian mothers stand in stark contrast. In Washington state, the number of low birth weight babies born to Southeast Asian mothers dropped by $25 \%$ between 1980 and $1986(\mathrm{Li}, \mathrm{Ni}$, Schwartz et al, 1990). In fact, the actual birth weight increased an average of 95 grams during that time. When subsequent births were examined, the number of low 
birth weight babies declined by 55\%. An increased age, a male child, more than one pregnancy, and an employed father all were correlated to a heavier newborn. Rumbaut (1996) found the same pattern in a cohort of Southeast Asian mothers in San Diego. His results were explained by very low rates of smoking, and alcohol and drug use among pregnant Southeast Asian women. A similar pattern was reported in Belgium among immigrants from North Africa (Buekens, Masuy-Stroobant, Delvaux, 1998). In that study, however, the infant mortality rate was higher among North Africans than among indigenous Belgians.

The conclusion drawn from these findings has generally been that acculturation acts as a marker for "certain values, beliefs or lifestyles" (Scribner and Dwer, p. 1266) rather than influencing birth weight directly (Cobas et al., 1996; Sing \& Yu, 1996). Some of these acculturation-related behaviors included smoking, using alcohol and marijuana/cocaine during pregnancy, formula feeding rather than breast feeding, living with the father of the baby or with other fanily members, and having a wanted baby (Cobas et al., 1996). When studies of infant birth weight of infants from different ethnic groups are considered, the relationship of infant birth weight and acculturation appears to vary, based on characteristics of individual ethnic groups.

\section{Acculturative Stress}

Acculturative Stress is stress due to the acculturation process that results in "anxiety, depression, feelings of marginality and alienation, heightened psychosomatic symptoms, and identity confusion" resulting in "anxiety, depression, feelings of marginality and alienation, heightened psychosomatic symptoms, and identity confusion" (Williams \& Berry, 1991, p. 634). Stress from acculturation has been widely reported among Southeast Asian refugees (Adler, 1995; Beiser, 1990; Gold, 1992; Matsuoka, 1993; Westermeyer, Neider, \& Vang, 1984); however, among refugees, it is difficult to ascertain how much of their stress arises from traumas experienced before and during migration, and how much comes from learning how to live in a new environment.

Voluminous numbers of studies have been published on the post-migration experiences of Southeast Asian refugees. Many findings that have been published come from ongoing examinations of massive data bases in Vancouver, British Columbia (Beiser, 
1990), California (Chung \& Kagawa-Singer, 1993), Minneapolis, Minnesota (Westermeyer, 1986), and Boston (Mollica, Wyshak, \& Lavelle, 1987). The early literature was primarily clinical in nature, for the need to describe the mental health needs of the communities, identify the incidence, and develop treatment or supportive resources was acute. Those studies identified that major post-migration stressors of a lack of competence in English, unemployment, low socioeconomic status, and separation from family contributed to mental health problems (Beiser, Tumer, \& Ganesan, 1989; Hauff \& Vaglum, 1995; Nicassio, 1985; Westermeyer, Vang, \& Neider, 1983). In addition, older age has consistently been linked to increased depression, as has the female gender (Chung \& Kagawa-Singer, 1993; Hinton, Tiet, Tran, \& Chesney, 1997; Liebind, 1996).

Although many researchers identified various sources of acculturative stress. Nicholson's (1997) research was unique in that her path analysis was able to delineate between premigration and post-migration predictors of PTS, anxiety, and depression. Current stress represented many post-migration stressors, including such things as housing, employment, language, education, loss of clan networks, loss of welfare benefits, experiences of personal violence (burglary or rape), or insufficient finances. Coefficients for current stress and PTSD $(\underline{r}=.40 \underline{p}<.001)$, anxiety $(r=.35 p<.001)$, and depression $(r=.38 \mathrm{p}<.001)$ were greater than for any other predictor variables.

Williams and Berry, (1991) provided another excellent discussion of the various causes of acculturative stress. They asserted that the degree of acculturative stress was related to the degree of acceptance by the new community, choices made relative to joining local community life, and the degree to which expectations about interactions with individuals in the new community were fulfilled. They reported that a key predictor for acculturative stress was a felt sense of personal control over the acculturation process.

Other factors influencing acculturative stress included how long the refugee had lived in the new society, how pluralistic the new community was, the availability of a supportive group from the same ethnic background, and individual characteristics of the refugee. Those who felt marginalized, or attempted to remain separate from the surrounding culture, were reported to have experienced more stress. Those who were isolated from supportive ethnic networks, those who had a decreased socioeconomic 
status, and those who found themselves excluded from resources by virtue of personal characteristics (race, religion, immigration status) or government policies also experienced more stress (Williams and Berry, 1991).

In refugee populations, an ability to become functional in the dominant culture has been overwhelmingly related to an ability to speak English and become employed and financially self-sufficient. Without an ability to speak English it is very hard to be employed. Unemployed people are frequently relegated to the periphery of society, unable to participate or function in the general culture. Welfare benefits, including temporary refugee benefits, have become the safety net for those who could not become financially self-sufficient during their initial years in the United States (Chung \& Bemak, 1996: Downing, 1986; Gold, 1992; Rutledge, 1985).

Receiving welfare benefits, however, has consistenty been related to a higher degree of psychological distress in Southeast Asians (Chung \& Bemak, 1996: Chung \& Kagawa-Singer, 1993). Chung and Bemak compared those who had contirucusly received welfaie benefits, those who no longer received benefits, and those who had never received benefits. Seventy percent of Vietnamese and $89 \%$ of Hmong participants had used weltare at some point. Of both groups, $46 \%$ had remained on welfare the whole five to six years since arriving in the United States. Hmong respondents reported the highest overali levels of psychological distress. Education and literacy rates matched overall levels of distress. Seventy percent of the Vietnamese population spoke English, 96\% reported literacy, and the mean education level was 10.5 years. They demonstrated lower acculturative stress. In contrast, only $34 \%$ of the Hmong spoke English, $38 \%$ reported literacy, and the mean education level was 3.1 years, They demonstrated the highest stress levels of all four groups of Southeast Asians. In contrast, Matsuoka (1993) found that the more highly educated Vietnamese experienced more acculturative stress than those with less education. He considered the dissonance between expected employment status and actual work available to be the explanation for that finding.

Many Southeast Asians reported that their new neighbors were hostile, suspicious, and resentful toward them (Rutledge, 1985; Gold, 1992; Calderon, 1986). Liebkin (1996) reported that such experiences resulted in increased levels of anxiety. Some communities 
used common religious ties to provide new support systems for Vietnamese that could replace the missing clan system (Rutledge, 1985). For those who who were not Christian, greater feelings of alienation were reported (Matsuoka, 1993: Beiser, Turner, \& Ganesan, 1989). Hmong people were generally not Christian, therefore the religious community contributed to increased stress levels rather than ameliorating them. Westermeyer (1986) reported that some sponsoring Christian churches attempted to coerce Hmong people to convert to their particular Christian beliefs, with the result that many Hmong were distressed to the point of attempting suicide.

For women, some of the stress of acculturation has been around expectations of parenting (Meleis, 1991). Parents socialize their infants and children to relate to society in ways consistent with parental goals in particular societies (Foss, 1996; Harkness \& Super, 1996; Jackson, 1993). When individuals or families move to a new country and culture, they take an internalized script with them. Often this script is different from the new society, and at times conflicts with what is found there. Parental response will be to either change parenting patterns, resist changes, or integrate traditional parenting patterns with aspects of the new culture. Studies about differences in parenting practices reported by Henderson and Brown (1987), Jambunathan (1995), and Rossiter, Ledwidge, and Coulon (1993) illustrated such parental responses in Vietnamese and Hmong cultures.

While acculturation has been related to infant mortality, infant birth weight, PTSD, anxiety, depression, and to parenting behaviors, how acculturation influences sensitivity to an infant has not been addressed. This study examines the relationship between acculturation and maternal sensitivity.

\section{Summary}

No research studies were located that described or investigated how various immigrant groups experience the overiapping transitions of transition to parenthood and transition resulting from migration. Maternal sensitivity, postraumatic stress, and acculturation have all been investigated individually. The relationship between traumatic events and PTS, anxiety, and depression in the general refugee population has been well established (Abe, et al., 1994; Chung \& Kagawa-Singer, 1993; Gonsalves, 1992; Hauff \& 
Vaglum, 1995; \& Mghir, et al., 1995), however no studies have examined how posttraumatic stress affected maternal sensitivity. Many studies supported the negative effects of maternal depression on maternal sensitivity in the general population, but none of those studies has been conducted in refugee populations. In addition, no studies reported how acculturation influences maternal sensitivity. Research did support the notion that acculturation negatively influences infant birth weights in some cultures, but in the Southeast Asian population acculturation appears to positively influence birth weight. Based on the literature review, increased levels of (PTS) or its components of anxiety and depression, and a low degree of acculturation were expected to be found in this population of Vietnamese and Hmong mothers. If a higher level of PTS, depression, or anxiety was reported by a mother, then it was expected that her sensitivity to her infant would be decreased. If a mother reported a higher degree of acculturation toward the dominant society, it was also expected that her sensitivity to the infant's cues would also be diminished. Based on the literature, there was expected to be some correlation between acculturation and PTS. The present study investigated the relationships among posttraumatic stress, acculturation, and maternal sensitivity in Vietnamese and Hmong families who immigrated to the United States since 1975.

A consequence of a lack of research with mothers and infants has been an absence of a discussion of affective and appropriate research methods. Due to that dearth, the development of a methodology appropriate to characteristics of Vietnamese and Hmong mothers was of paramount concern. As detailed in chapter three, the methodology reflected the paculiar characteristics of the population under study. 


\section{CHAPTER 3}

\section{Methodology of the Study}

The purpose of chapter three is to describe the methodology used to conduct the research study. First, the sampling procedure is described, including entree into the Vietnamese and Hmong communities, a description of the sample, and a discussion of sample size. The research design with the use of interviews as the method for data collection is then addressed. Because the data were collected in the home, the visit protocol and the use of interpreters are discussed. Next, the protection of human subjects is addressed, followed by a description of the instruments, including the procedure for translation of the consent, and the Suinn-Lew Acculturation Scale (SL-ASIA). Finally, the methodology for analysis of the data and the assumptions and limitations of the methodology are addressed.

\section{Sampling Procedure}

\section{Entree into the community}

Based on the model described by Hautman and Bomar (1995), an assumption was made that the investigator would need a personal introduction into the Vietnamese and Hmong communities by trusted leaders, such as clergy or physicians. Only then could recruitment of participants begin. It was expected that once the trust and endorsement of individual leaders was obtained, members of corresponding religious communities or medical practices would be more likely to consider participation. This assumption was confirmed.

The investigator contacted Vietnamese physicians in many communities of San 
Diego who agreed to distribute English/Vietnamese and English/Hmong flyers (Appendix B) and recruit from among their well-child populations. No participants resulted from those contacts. The Union of Pan Asian Communities agreed to distribute flyers and recruit among Vietnamese and Hmong mothers who requested assistance locating child care, however, again no participants resulted from that contact. The faculty sponsor of the Asian nurses association at San Diego State University agreed to distribute flyers to student members. Three participants resulted trom that contact. Several clergy agreed to recruit in their congregations. The majority of Vietnamese participants came from those contacts.

\section{Vietnamese Participants}

The investigator knew a Vietnamese clergyman for several years as a colleague of her clergy husband. This man and his family became primary key informants in the Vietnamese community throughout the research process. Other key informants in the Vietnamese community were the investigator's hairdresser and her husband, and the Vietnainese interpreters who accompanied the interpreter on home visits. The tirst clergyman provided links to other Protestant and Roman Catholic clergy, with the result that a total of ten were contacted. Out of that number, four clergy recruited participants for the investigator. Many key informants, including two who identified themselves as Buddhist, uniformly discouraged the investigator from contacting the Buddhist monks in the area. The only English-speaking monk had left the area, and they believed the language barrier was too great. They also reported that people only congregated at the temple for festivals, and they thought the investigator would have difficulty recruiting during festivals.

The investigator offered to reciprocate to each participating faith community by offering to teach health education classes on a topic of their choice, but all clergy declined. They commented that each family "has their own doctor and learn all they need from him." They stated that because the focus of the researcher on mothers was good for the Vietnamese community, they would be happy to assist without further compensation.

After explaining the study to participating clergy, the investigator attended several church services when that was suggested. Although not introduced to the congregation formally, this allowed potential participants to note her presence and have a frame of reference when subsequent telephone contact was made. Clergy then gave the investigator 
names and telephone numbers of families who they thought met criteria for participation and might consider participating in the study. English-speaking ability was indicated in the referral. The investigator then called the family to explain the referral and the study, or used an interpreter to make the telephone call from a script. The same procedure was followed for referrals from non-clergy sources.

\section{Hmong Participants}

No Hmong clergy were located. A group of Hmong associated with one church requested substantial financial reimbursement for each family who participated. The clergy contact for that group reported that this particular group of Hmong community leaders stated they were tired of other penple profiting from books written about the Hmong while the Hmong did not share in those benefits. Consequently, Hmong members of that religious community had decided they would only participate in research projects ix paid substantial funds in advance.

A nursing student colleague introduced the investigator to a classmate from another major who was Hmong. He endorsed the study and referred the investigator to his sister for assistance with recruitment. With the assistance of her family, this key informant first translated the flyers and consents into Hmong, then personally introduced the investigator to participants who had agreed to the interview as well as to other potential participants in the community. In addition, she interpreted for the investigator on home visits when requested to do so by Hmong mothers.

\section{Description of the Sample}

Once entree to the community was achieved, purposive sampling techniques and snowballing were used. Although this method introduces bias, it has been supported as an effective means to access hidden populations (Faugier \& Sargeant, 1997). During the review of the literature, it was noted that refugee mothers have not been the focus of study about maternal sensitivity, symptoms of PTS or acculturation. For this neglected population, purposive sampling and snowballing were successful in providing access to that neglected population. Criteria for participation included: (a) presence of a healthy infant between 1-30 months, (b) presence of both mother and infant for the interview, (c) history of migration to the United States since 1975, and (d) informed willingness and consent to 
participate in the study.

Most of the participants for this study lived in or near two major regions of San Diego, but many were scattered around other areas of the city. The two major areas that contributed most participants had less expensive apartments and small houses as well as many public housing units.

Churches in those two major areas of San Diego had clergy designated for specific immigrant groups, including Vietnamese, Hmong, Hispanic, and others. In addition, each church group also had recognized leaders from their own ethnic group. Vietnamese participants reported a close supportive network among immigrants who belonged to the same faith community. Hmong participants reported a close supportive relationship based on clan membership as well as church affiliation.

\section{Sample Size}

The original study proposal included an infant variable as a second endogenous variable. For a two-group sample with a standardized effect size (d) of .50 , alpha set at .05 , and the power established at .80 , a minimum of 62 participants were needed (Hinkle, Wiersma, \& Jurs, 1994). When it became clear that due to time and resource constraints it would not be possible to recruit sufficient participants for statistical analysis of two endogenous variables, data about the infant was not included in any analyses. Maternal sensitivity remained as the sole endogenous variable.

Determination of an appropriate effect size for maternal sensitivity in Vietnamese and Hmong mothers was problematic. The only studies located about maternal sensitivity in any Southeast Asian group were conducted with Hmong mothers. Maternal sensitivity scores were reported in only one of the studies. On a scale of $1-9$, they were between 8 and 9 (Oberg, et al., 1986). Such extremely high scores contrasted with a recent large study with primarily European-American participants that reported a mean score of 6.6 on the same Sensitivity versus Insensitivity to the Baby's Communication Scale (Heermann, Jones, \& Wikoff, 1994). Based on these findings, it was assumed that a score of seven or lower would indicate that a significant change had taken place in the mother's sensitivity toward her infant. That change was considered to correspond to a medium effect size, so the standardized effect size was established at 0.50 . Significance was set at 0.05 , and 
power for the Sensitivity versus Insensitivity Scale was established at .80 . Based on these parameters, a minimum of 27 participants were needed for this study (Hinkle, et al., 1994).

A total of 43 mothers discussed the project with the investigator. Of that number, 33 agreed to participate. The most frequent reason given for declining was insufficient time available to meet with the investigator. Of the 33 participants, one had both the HSCL-25 and SL-ASIA missing. That case was deleted from the sample. Two others were missing only the HSCL-25, and the remaining 30 completed all parts of the interview. The final sample size was 32 .

\section{Research Design}

A descriptive correlational (Burns \& Grove, 1993) research design was used. Figure 2 delineates the theoretical relationships. Depression and anxiety are indicated to be two domains of PTS that decrease maternal sensitivity when present in the mother. Higher levels of acculturation are also shown to decrease maternal sersitivity. Interrelationships are shown among all the predictor variables. There was no intent to manipulate any of the variables, only to investigate relationships as they occurred in the designated populations.

All data were collected by the primary investigator, who interviewed each mother in her own home or that of another family member. The interview method of data collection has been generally preferred when conducting research in immigrant communities (Lipson, 1992), and conducting the interview in the home setting has been more frequently reported when community based samples of refugees were used (Nicholson, 1997; Rousseau, et al., 1997). The interview format was based on public health nursing standard practice. All contacts were at a time convenient to the mother and in a space of her choice that provided appropriate privacy.

Initially, two visits to the home were made at a one or two-week interval. Key informants from both Vietnamese and Hmong communities recommended consolidating the content of both visits into one as a means of increasing the number of participants. This was done, and the final two thirds of the sample received only one visit. The order of the visit was identical for both the one-visit and two-visit pattern. 


\section{Use of Interpreters}

Because an overriding concern throughout the study was to optimize the validity of the responses, each participant was given the opportunity to use an interpreter of her choice. Most mothers requested that the investigator bring an interpreter with her. Five mothers chose to use another family member to interpret. Professional interpreters charged in excess of $\$ 2500$ per hour. This was considered prohibitive for this study, so a less expensive source of interpreters was sought.

Vietnamese interpreters were recruited through the work-study program at the University of San Diego (USD). Four participants were visited by the investigator, who brought a different USD interpreter to each family. The students' time schedule was limited, and they had difficulty going with the investigator at times convenient to the family. That group of interpreters was supportive of the study and provided valuable leedback about Vietnamese culture and the recruitment process used in this study. One participant interpreted for four other mothers after the completion of her interview.

For the Hmong participants only one interpreter was used. As a member of one of the clans, she had entree into a major section of the local community. She also referred all questions about the study, as well as questions about specific items on questionnaires, to the investigator.

With a variety of interpreters, reliability was a concern. Before making the visit,the investigator explained the procedure to the interpreter, emphasizing her role as only a conduit of information in two directions. When questions arose, the interpreter relayed the question to the investigator rather than answering it herself. After leaving the home the interpreter relayed additional social information she gleaned while in the home. Although unrelated to the study, that information added insight to the investigator's understanding of this population of mothers.

\section{Visit Protocol}

The home visit followed the same pattem throughout. Prior to the visit, the need for an interpreter was determined, and the source of that interpreter ascertained. If needed, the designated interpreter then accompanied the investigator to the home. After observing appropriate social exchanges, the investigator explained the study, its purpose, and the 
measures to be used. Consent for participation was sought from the mother (Appendix C). Some mothers waited until their husbands had read the consent form before assenting. One mother declined after consulting with her husband. She only stated that he did not want her to participate. The voluntary nature of participation was stressed, and the phone number of the investigator was included in the consent form.

Demographic data were collected from all participants (Appendix D). As a means to standardize an interaction between the mother and infant, each mother was given a task that required interaction with her infant. A set of brightly colored nesting cups was given to her with instructions to show her baby how to play with them. The investigator quietly observed the interaction for five minutes. Throughout the home visit, the investigator observed the mother and infant interaction, using the Sensitivity vs. Insensitivity scale developed by Ainsworth (1976). Consistent with public health nursing practice,the Ainsworth scale (Appendix E) was scured immediately after the investigator left the home and community.

At the conclusion of the activities with the infant, other family members frequenuly removed the infant from the mother's supervision. When other family members were present, they usually left the room during the questionaire-based interviews. When the mother indicated she was ready, the Suinn-Lew Acculturation Scale (SL-ASIA) (Appendix F), then the Hopkins Symptom Checklist-25 (HSCL-25) (Appendix G), were read to her by either the interpreter or the investigator and her responses recorded by the investigator. At the onset, the mother was encouraged to ask for clarification of unfamiliar terms. Many mothers did need clarification of terms in both scales. They were reminded that their responses were voluntary and they could choose not to answer any part of either questionnaire. Five mothers chose not to answer the question about sexuality that was part of the HSCL-25.

By leaving the questionnaires to the end, it was hoped the mother would have become sufficiently comfortable with the investigator to allow candid and accurate responses. Usually family members became distracted and left when the interactions with the infant were over. If family members remained, the personal nature of the HSCL-25 was reiterated to allow the mother to arrange increased privacy. 
The investigator used the HSCL-25 that matched the family's original language. If the score indicated the presence of clinical depression or PTS, the mother was informed of the score and its significance, and then encouraged to seek treatment from her medical provider or a mental health resource. This protocol followed standard public health nursing practice. At that time, the investigator offered to give the mother a copy of the HSCL-25 for her to give to her medical provider.

Only one mother refused the copy of the tool because she stated that her provider "was aware of all of the problems" she was having. All but one mother with scores indicating clinical levels on the HSCL-25 indicated that she had discussed her problems with the medical provider but wanted to take the tool with her to the docter. They all stated they wanted a copy of the tool because it was hard for them to explain to the doctor their problems. Some went on to say that the doctor misunderstcod them, or the family member they took with them to interpret did not always understand her problems. and thus misconmunicated or misrepresented them to the provider. They stated that the HSCL-25 wculd help them communicate directly with the physician. The investigator copied the HSCL-25 and mailed it to the mother within one day. A total of eight mothers were given copies of their HSCL-25.

At the end of the visit, the investigator offered to assist the family to identify any needed resources. The most common assistance requested was location of affordable child care. The investigator explained possible resources and left with them the telephone numbers of the Child Care Resource Center and the Child Care Placement Service of the Union of Pan-Asian Communities. At that time, a small age-appropriate educational toy was left for the infant, and health promotion materials from the Department of Health Services (Appendix $\mathrm{H}$ ) were given to the mother as a thank-you for participating. No time was spent explaining the materials other than briefly describing them and stating that those particular materials were commonly given to families by public health nursing personel in clinic or home settings. They were encouraged to take them to their personal physician or health department center if there were questions. 


\section{Protection of Human Subjects}

Prior to any data collection the research plan was approved by the Committee for Protection of Human Subjects at the University of San Diego (Appendix D. Because refugee mothers and infants represent an especially vulnerable population, particular attention was paid to potential risks and risk management. The study was explained on the telephone prior to obtaining an address for a home visit. At the beginning of the visit, the study was again described and the consent reviewed. An assumption was made that if the participant had invited the investigator to visit her at home, she was consenting to participate. Key informants from both groups had informed the investigator that obtaining written consent would be problematic; consequently, participants were not required to sign the consent. A copy of the consent form, signed by the investigator and in the language requested, was left with the mother (Appendix $\mathrm{C}$ ). The consent form then becane a contract, representing the investigator's commitment that only the items included in the consent would be included as part of the visit.

The mother was informed that she had the right to withdraw from the study at any time. Two mothers declined to answer the HSCL-25. One mother said she did not wart to talk about any possible problems. The other had a male guest who came at that time, and she had no other place to go to complete the visit in private. Concerns about privacy were addressed directly when other family members were present. The husband frequently left at the beginning or during the SL-ASIA. The children were usually excluded by the mother during the questionnaires. If family members remained at the beginning of the HSCL-25, the investigator reiterated the personal and medical nature of the questions. At that point the investigator followed the wishes of the mother. If she wanted to terminate the interview, that was done. If she wanted to complete the interview, it was usually conducted quietly in one comer of the room.

Another concem was the need to inform each mother that the investigator was mandated to report child abuse; a point that was clearly stated on the consent form. Given a history of Child Protective Service involvement when traditional discipline methods were used and when signs of coining were seen, key informants confirmed that the index of suspicion regarding mandated reporters was high in both communities. As a result, the 
investigator stressed her public health nursing history with members of both groups and her respect for the parenting skills of both groups. Vietnamese and Hmong community resource people also informed potential participants that the investigator was familiar with Vietnamese and Hmong ways of child care and child discipline.

All interviews were coded, then separated from names and addresses of the participants. All data and identifying information were kept in a locked file in the investigator's home.

\section{Instruments}

The following instruments were used for collecting data: (a) Ainsworth's Sensitivity vs. Insensitivity to the Baby's Communication Scale (Appendix E), (b) The Suinn-Lew Asian Self-identity Acculturation Scale (Appendix F), and (c) The Hopkins Symptom Checklist-25 (Appendix G). Demographic information was collected at the beginning of the home visit. This section first addresses methodological concerns when conducting cross-cultural research, followed by a description of the demographic data that was collected. Finally the psychometrics of each instrument is presented in the order in which it was used.

\section{Cruss-cultural Instrumentation}

Ferketich, Phillips and Verran (1993) identified three essential components of instrumentation in cross-cultural research. The first was that there should have been qualitative work to "inform the researcher about the meanings and language used by the target population to describe the phenomenon" (p. 228). The second was to decide if the goals of the research were comparative or operational. Comparative research looks for a specific construct across cultures without major concern for the appropriateness of that particular construct in another culture. Translation occurs literally even if the meaning in the second culture is obscure or inappropriate. Operational research, on the other hand, seeks to identify a common construct across cultures. The goal of translation is to find an equivalent meaning for the construct in the second culture (Jones \& Kay, 1992). The third component was to "match translation methods with the goals of the research" (Ferketich, et al., 1993). These three components are addressed here. 
The investigator had not conducted qualitative research work before embarking on this study. However, her years of practice in Vietnamese and Hmong communities as a public health nurse exposed her extensively to belief systems, family and cultural lifeways, health care practices, and values that contributed to an appreciation or the perspective of each group. In addition, time had been spent with primary key informants over months, and in some cases years, listening to stories about life in Vietnam or Laos, the escape, the difficulties of resettlement, and hopes for the future. Their perspectives, ideas and suggestions were incorporated into the research design and method of data collection. In addition, the literature that was reviewed is replete with information about the history and traditions of both cultures.

As the term was defined by Ferketich, et al. (1993) and Jones and Kay (1992), the cross-cultural goal of this study was operational. The intent was to identify the degree to which the concepts under investigation were indeed present in the population under study. Each instrument was selected based on its reported ability to identify a particular construct in other cultures.

Consistent with the third component of cross-cultural research (Ferketich et al., 1993), translation methods for the recruitment tlyer, the consent, and the SL-ASIA were selected with the goal of making them conceptually equivalent with the English version. The committee method for translating all materials was selected (Brislin, Lonner, \& Thorndike, 1973). In this method, a group of two to three people together translate the tool from English into their native or the target language. Another person or group then reviews the translation to evaluate the accuracy of the translation. Disagreements are resolved by consensus of the two groups. The disadvantage of this method is that the investigator has little or no control over misconceptions about terms that may be common to both groups of translators. The advantage of using the committee approach is that it consumes less time and money. In this study, the investigator was working within time constraints and was restricted financially to the use of volunteers who had to work around other commitments, thus the committee approach was the most feasible.

Translation Protocol

In the Vietnamese community two different bilingual key informants independently 
formed a translating committee consisting of family members and friends to translate items from English to Vietnamese. The investigator consulted with both groups regularly to answer language and conceptual questions. Both translations with the English language original were then submitted to a third independent bilingual person with experience translating for health care professionals. She reviewed both translations and chose specific translations she considered to be the most true to the English concept. The modified version was then resubmitted to the primary key informant, who had been a school teacher in South Vietnam, for conceptual validation. The result of that final conference was the version of materials used for the study.

In the Hmong community, only one key informant was located. She formed a committee of family members and bilingual friends who translated the materials. The key informant as well as all of the Hmong participants used the same Hmong dialect. Again, the investigator consulted frequently with her to answer language and conceptual questions. The use of only one committee increased the possibility of error in the translations. The key informant who translated the materials also interpreted for the investigator. It was hoped that the consistency between translator and interpreter decreased the chance of error in communicating the concepts in the materials.

\section{Translation of Flyers and Consent}

The recruitment flyers (Appendix B) and consent form (Appendix C) wcre translated into Vietnamese and Hmong as previously described. All translators expressed concern about the section in the consent that stated the investigator was a mandated reporter of child abuse. Minor modifications to the wording were suggested by both groups to clarify and emphasize the legal nature of the requirement to report child abuse. Those suggestions were implemented. Other suggestions simply made the language more culturally congruent. Most of the recommended modifications in wording of the English phrases were adopted. Subsequent use of the flyers and consents confirmed the wisdom of incorporating suggested changes.

The process of translation provided insight into social structures particularly within the Vietnamese community. The investigator was told by all translators and interpreters that slightly different Vietnamese dialects were spoken in the local Vietnamese community. 
These dialects varied depending on the region of Vietnam where the speaker originated. Some of the differences in wording or phrasing of the flyer and the consent were due to those differences. In addition, the investigator was informed that the original social class of the speaker dictated additional differences in wordings. In those few instances when all three groups continued to disagree about a particular wording or phrase, the investigator chose to maintain propriety and and a positive collaborative relationship with the primary key informant by accepting his opinion. During initial interviews with potential clergy informants and recruiters, each one identified a phrase or word that he suggested be "corrected." Notes were made of each suggestion, and subsequent consultations about the suggested changes were made with the primary key informant. Only one additional change was made in the recruiting flyer.

Demographic Data

The demographic data (Appendix D) were collected in an artempt to provide an overview of the family structure, maternal-infant hedth history, educational background, language preferences, and migration history of the family. Responses were written on the demographic form in the presence of the family.

Questions 1-6 asked about the ages of the family members, the parental occupations, the educational level of both parents. Questions 7-10 related to the reason for migration, who made the decision to migrate and where the extended family was located. Questions 11-14 asked about the health status of the mother and her infant. A final question was open-ended, asking for information about any other aspect of maternal-infant interactions that the respondent wanted to provide.

\section{Matemal Sensitivity}

The mother's sensitivity to her infant's communication was measured by one item from a set of 26 observational scales developed by Mary Ainsworth (Appendix E) and standardized in an attempt to systematically measure maternal-infant interactions and the degree of an infant's attachment to his or her mother (Ainsworth, 1976). Sensitivity vs. Insensitivity to the Baby's Communication is one of a subset of four global scales measuring the mother's side of the interaction between infant and mother. It measures awareness of the infant's signals, the ability to interpret them accurately, and the 
appropriateness of the response (Ainsworth, 1976). The mother's behavior is ranked on a nine-point Likert scale with one as the lowest score possible and nine as the highest (Ainsworth, Blehar, Waters, \& Wall, 1978). Behaviors are described in detail for each of the odd numbers on the scale, although a score may be assigned to a mother at any point on the scale. The investigator rated the mother at the end of the home visit using Ainsworth's five descriptors and nine-point scale. The score derived was the Maternal Sensitivity score.

Ainsworth developed her observational scales in Uganda (Ainsworth, 1967) and validated them in Baltimore (Ainsworth et al, 1978). She spent many hours observing infants and their care givers in their homes and recording her observations. This tool was recently reported to be a valid indicator of maternal sensitivity in situations of extreme poverty in developing societies (Valenzuela, 1997) and has been deemed valid in a Hmong population in the United States (Muret \& Wagstaff, 1989; Oberg, 1986). The Ainsworth scales have now become generally accepted as a valid measure for maternal sensitivity in different cultures in both laboratory and home settings (Ainsworth, et al., 1978; De Wolff and van IJzendoom, 1997).

The degree of a mother's sensitivity to her infant is a concern throughout the first three years of life (Ainsworth et al., 1978; Bowlby, 1982). Measurement of a mother's sensitivity to her infant has generally been paired with measurement of the infant's attachment to the mother. Consequently, the majority of reports of maternal sensitivity have been for mothers of 12 month-old infants (De Wolff \& van IJzendoorn, 1997). When other infant outcomes, such as number of accidents (Oberg, 1986), chronic undernutrition (Valenzuela, 1997), or gender-based communication patterns (Biringen, Robinson, \& Emde, 1994) were paired with maternal sensitivity, reports of maternal sensitivity in mothers of older children were found.

Observational rating scales require the observer to rate the participant along a continuum that describes behavior at various points. Agreement between the observers, or interrater reliability (Waltz, Strickland \& Lenz, 1991), is essential. Interrater reliability for maternal sensitivity has been reported to be high between trained observers (Biringen, et al., 1994; Heermann, et al., 1994). For the current study, ratings for interrater reliability were obtained by observing videos of maternal-infant interactions and comparing the 
expert's score of maternal sensitivity to the investigator's. The procedure outlined by Heermann et al. (1994) was followed. Two sets of previously rated videos were provided by the chair of this dissertation committee, who was also one of the authors of Heermann's study. The first set was studied and rated several times by the investigator. A second. larger set of videos was then obtained from the same data-base.Videos for ages one month, six months, and twelve months were viewed and rated. Scores were then compared with the expert's ratings, and were considered to agree if there was no more than one point difference. The investigator's overall agreement with the Heermann et al. rating of the videos was above $90 \%$ at that time. An agreement rate of $90 \%$ is considered acceptable (Waltz, et al., 1991). The Kappa statistic for 12 months was .52, indicating that the agreement was not just chance (Waltz et al). Kappa could not be computed for one or six months because the sample did not include scores for all points between 5-9 on the scale (Norusis, 1994).

After a time lapse of four months, and half-way through the interviews with participant families, the same second set of maternal-infant interaction videos was again viewed and rated. At that time the interrater agreement was above $89 \%$, but the Kappa statistic for 12 months was .40 , and for one month was only .17. Intrarater reliability refers to the consistency of scoring between two series of observations by a single rater (Waltz, ct al., 1991). Intrarater reliability for the two viewings was above 90\%, and Kappa statistic was .48 for the 12-month group.

Suinn-Lew Self-identity Acculturation Scale (SL-ASIA)

The Suinn-Lew Self-identity Acculturation Scale (SL-ASIA) (Appendix F) was selected to measure acculturation levels in this study because it was created specifically for Asian populations by Suinn, Rickard-Figueroa, Lew, and Vigil in 1987. This group of researchers brought an emic, or insider's, perspective to acculturation among Asian groups, therefore using terms, phrases, and examples that would have been familiar to Asian groups (Suinn, et al., 1987). It has since been used in various Asian populations, including Vietnamese (Lese \& Robbins, 1994).

The SL-ASIA is just one of many tools that have been developed for specific ethnic groups in an attempt to measure acculturation. Most measures have focused on language 
use (Celluar, Harris, \& Jasso, 1980; Mavreas, Bebbinton, \& Der, 1989; Suinn, Ahuna, Khoo, 1992), however, the importance of cultural identification as well as language has been increasingly recognized and included in more recent scales. The purpose of the SLASIA was to measure both language and cultural identification. It was modeled after a scale developed by Cellar, Harris, and Jasso's Acculturation rating scale (1980) for Mexican Americans (ARSMA).

The SL-ASIA consists of 21 questions, covering six areas: language (four questions), identity (four questions), friendships (four questions), behaviors (five questions), generational/geographic background (three questions), and attitudes (one question). The responses are rated on a five-point Likert scale. Individual scores are obtained by summing the total responses and dividing by five. A lower score means the respondent identifies more with the traditional Asian culture. while a higher score means the respondent is more acculturated toward the dominant culture found in the United States.

Internal reliability (Cronbach's alpha) was 0.88 when the instrument was first developed. When validated in a second study in 1992, Cronbach's alpha was 0.91 . Concurrent validity was reported between the scale and education in the United States $(r)$ $.61)$, time lived in the United States $(r=.56)$, age when arrived in the United States $(r=$ .49), how many years lived in non-Asian communities $(r=.41)$ and one's own assessment of acculturation $(r=.62)$. Factorial validity was reported between the ARSMA and the SLASIA. Three factors that accounted for $58 \%$ of the variance in the SL-ASIA also accounted for $58 \%$ of the variance in the ARSMA. The factors that distinguished them were "affinity for ethnic identity and pride, and food preference" but parts of the other factors were shared by the two scales (Suinn, et al., 1992, p. 1045).

In 1994, five questions were added to the scale. They were simply categorical in nature, covering three areas; values (two questions), behavioral competencies (two questions), and self-identity (one question). If the the five supplemental questions are used as part of the total score, the scoring of items 1-21 must be changed to categorical ratings. Different results could be obtained by this method. Dr. Suinn recommended that both approaches be used as a way to grasp more clearly some of the nuances associated with the concept of acculturation. In this study, all 26 questions were asked, but only the first 21 
questions were used to calculate the score. Since the investigator wanted to relate the degree of acculturation to maternal sensitivity, a ranked score was preferable.

The SL-ASIA was selected for this study because it included questions about social and behavioral aspects of acculturation as well as language abilities of the respondent. In addition, because both Vietnamese and Hmong populations have a common Chinese heritage, it was expected that the SL-ASIA would translate into Vietnamese and Hmong with greater ease than other available tools.

Although the SL-ASIA was developed to fit a broad cross section of AsianAmerican cultures, it has been primarily used with Chinese, Japanese, and Korean groups. When used in a Vietnamese population, it was not translated into Vietnamese prior to the study. Instead, individual items were translated to participants during the administration as needed, rather than translating it (Lese \& Robbins, 1994). Due to concerns about reliability if each item were translated for participants who did not read English, the SL-ASIA was translated into Vietnamese and Hmong prior to data cuilection.

The goal of translating the SL-ASIA into Vietnamese and Hmong was to tind equivalent meanings for the items. The same translation protncol that was described for translating the flyers and consent form was also used for translating the SL-ASIA from English into Vietnamese and Hmong. Few difficulties were reported by either Vietnamese or Hmong translators. Few differences occurred between the two committees in the Vietnamese group. All key informants recommended that in the items that listed various Asian cultural groups, only "Vietnamese" or "Hmong" be used as an example of an ethnic group. In response to their suggestions, this one modification was made.

Thirty-two participants completed the SL-ASIA. A standardized reliability coefficient (alpha) was computed for only the first 21 items. Eight participants did not answer all the questions (Six could not read or write, and two did not ever eat in restaurants), so the alpha was computed only on 24 completed questionnaires. The alpha coefficient was .85 , thus demonstrating a fairly high level of internal consistency. Participants who did not answer all questions were the ones who scored lowest on the scale. Had their questionnaires been included, it is possible a different alpha coefficient would have resulted. 


\section{Hopkins Symptom Checklist-25 (HSCL-25)}

The level of posttraumatic stress (PTS) was measured by the Indochinese version of the Hopkins Symptom Checklist-25 (HCSL-25) (Appendix G). The purpose of using this particular tool was to identify if psychic distress was present in mothers without arousing painful emotions. The Southeast Asian versions of the HSCL-25 have a history of allowing Southeast Asian patients to identify and rate their own symptoms of psychic distress without stimulating a recurrence of the traumatic event (Mollica. Wyshak, de Marneffe, Khoun \& Lavelle, 1987).

The notion of allowing patients to rate their own symptoms of emotional distress originated during World War I in an effort to speed up the processing of soldiers for military service. The first version of the self-rated HCSL was developed from the Comell Medical Index by Parloff. Kelman and Frank in 1954 (Derogatis, Lipman, Rickels, Uhlenhuth, \& Covi, 1974). That version was primarily used in psychotropic drug research. Gradually, the HCSL evolved threugh several versions and stages of testing in general patient populations as well as psychiatric patient populations. Eventually. a 58 -item scale was developed and standardized by Williams, Lipman. Rickels. Covi, Uhlenhuth and Mactson (Derogatss et al.). This complete form of the HSCL was composed of tive domains; somatization, obsessive-compulsive, interpersonal sensitivity, depression and anxiety. The largest proportion of PTSD diagnoses were found to be represented in the anxiety and depression domains (Derogatis, et al., 1974).

Hesbacher, Rickels, Morris, Newman, and Rosenfeld (1980) took a total of 25 items from the 58-item HSCL to form a shorter scale that could be used in general practice to identify patients with anxiety, depression, or PTSD. They took ten items from the anxiety sub-scale (feeling scared for no reason; feeling fearful, faint, dizzy, weak, nervous, or shaky; heart pounding; trembling; tenseness; headaches; times of panic or terror; and restlessness), 13 items from the depression sub-scale (low energy; blaming self; crying easily; loss of sexual interest/pleasure; lonely; hopelessness about the future; feeling blue; suicidal ideation; feeling trapped; excessive worrying; lack of interest in things; feeling everything is an effort; and feelings of worthlessness), and two items from the somatic sub-scale (poor appetite and sleep problems). The somatic sub-scale was folded into the 
depression sub-Scale, thus the HSCL-25 has only two sub-scales. Depression and anxiety were each scored separately and thus could indicate either depression or anxiety. Since those two domains together were identified as the primary components of PTSD, the combined total score of the two subscales became the PTS score.

The HSCL-25 has become very widely used by clinicians to identify psychic distress in clinical practice with refugees (Mollica. Wyshak, de Marneffe, et al., 1987; Mollica Wyshak, Lavelle, Truong, Tor, \& Yang, 1990). More recently, it has been used in community samples to identify the presence of anxiety, depression or as an indicator of distress (McKelvey, Webb, \& Strobel, 1996; Nicholson, 1997). Part of the reason for its popularity has been its reputation as an instrument that does not arouse emotions around past traumas (Mollica, Wyshak, de Marneffe, et al., 1987).

Mollica, Wyshak, de Marnetfe, et al. (1987) at the Indochinese Psychiatric Clinic, Harvard School of Public Health, translated the HSCL-25. into Vietnamese, Khmer. and Lao. It was translated and back-translated blind. by teams of bilingual and bicultural staff from the clinic and the local Southeast Asıan community in Brighton, MA. The result was a conceptual and semantic translation of the English language HSCL-25. After testing the translated version for one year in the clinic, it was revised, based on comments of staff and other "cultural expers" (p. 498). The final instrument was published by the Harvard Program in Refugee Trauma, the Harvard School of Public Health, and the Indochinese Psychiatry Clinic at Deaconess Hospital and is available from them in Vietnamese, Cambodian, and Laotian.

The English language 58-item HSCL has been reported to have a high level of validity (.86) between the physician diagnosis and the patient's self rating of distress. Validity in the Southeast Asian versions was established by evaluating the scales's sensitivity and specificity. The sensitivity of the three translations was established at 0.88 and specificity at $\mathbf{0 . 7 3}$. Interrater reliability for each of the three language groups was greater than 0.98 and test-retest reliability was 0.89 (Mollica, Wyshak, de Marneffe, et al., 1987). Values for Cronbach's alpha were not given, however Derogatis et al. (1974) reported an alpha of 0.86 for the sub-scale of depression, 0.84 for anxiety, and 0.87 for somatization in his 58 -item HSCL. 
Hmong people were not separated from the rest of the Laotian group by Mollica, Wyshak, de Marneffe, et al. (1987) when the Laotian HSCL-25 was developed. Because of differences between Laotians and the history, education, and lifeways of the Hmong, a Hmong version of the HSCL-25 was developed by Mouanoutoua and Brown (1995). This Hmong version of the HSCL-25 was translated from English into Hmong by the authors, then back-translated by community members fluent in both languages. When the authors and community translators were satisfied that conceptual and semantic equality had been reached, the instrument was tested in clinical and community groups in the Fresno, CA area. Sensitivity was established at 1.0 and specificity at 0.78 , with a total validity score of 0.89. Reliability scores were high. Cronbach's alpha was 0.97 , the split-half coefficient was 0.92 , and the test-retest coefficient was 0.89 . In a factor analysis, "headache" loaded higher on the depression scale than the anxiety scale where it had loaded in the Southeast Asian version developed by Mollica, Wyshak. de Marneffe, et al. (1987); however, it was not moved to the depression scale when the Hmong version was published.

Both the Southeast Asian and Hmong versions of the HSCL-25 have four possible responses. The response "not at all" is given a score of one, "a little" is scored as two, "quite a bit" is scored as three, and "extremely" is scored as four. An individual's score is obtained by summing the scores of all the responses and dividing by the total number of questions the respondent answered (Mollica, Wyshak, de Marneffe, et al. 1987). The use of 1.75 as the point of clinical symptomatology dates to Winokur et al. (1984), who reported that patients with ratings below 1.75 "rarely developed symptoms of distress" during the month after completing the HSCL-25, whereas those with higher scores did. The score of 1.75 has remained as the cutoff point for clinical levels of symptoms, although clinicians continue to question if that score is valid in Southeast Asian groups (Nicholson, 1997; Mollica, Wyshak, de Marneffe, et al., 1990; Mouanoutoua \& Brown, 1995). Still no recommendation for a different cutoff point has been made.

Thirty participants completed the HSCL-25. Cronbach's standardized adjusted alpha was .93. It was based on 25 responses, as five of the thirty participants did not answer all of the questions. Thus for this population, the HSCL-25 was a robust and reliable measurement tool. 


\section{Analysis of data}

All analyses were computed using the Graduate Pack Statistical Program for the Social Sciences 6.l (SPSS) that was formatted for the Macintosh. Data and demographics were tirst entered into the computer and checked for accuracy. Frequencies and descriptive analyses were calculated and plotted. Anxiety was found to be positively skewed. Correlations ( $\mathfrak{r}$ ) among demographics were pertormed, and those that were highly correlated were either collapsed into a single demographic variable or deleted in favor of the more conceptually sound demographic variable. T-tests with the Levine statistic were then calculated to determine if there were significant differences between the two ethnic groups in both the demographics and the research variables. Finally, multiple regression was used to identify any predictive relationships. For some regression analyses, the probability was set at .10. Since no previous research provided infornation as to expected findings, results were considered to be exploratory.

To address Question 1 (To what extent do Vietnamese and Hmong mothers experience PTS?), means and Pearson correlations among PTS, anxiety, and depression were examined. To further ascertain their relationships, a forward multiple regression was then calculated using PTS as the criterion and anxiety and depression as predictor variables. Based on the results of the correlations and the regression, anxiety and depression replaced PTS in subsequent questions. To address Question 2 (To what extent does PTS, anxiety or depression decrease maternal sensitivity in Vietnamese and Hmong mothers?) Pearson correlations among maternal sensitivity, anxiety, and depression were examined. Again, a forward multiple regression was calculated using maternal sensitivity as the criterion and anxiety and depression as predictor variables. To address Question 3 (To what extent does acculturation decrease maternal sensitivity in Vietnamese and Hmong mothers?) Pearson correlations among maternal sensitivity and acculturation were examined. To address Question 4 (Among Vietnamese and Hmong mothers, is there a relationship between PTS, or its components of depression and anxiety, and acculturation?) Pearson correlations among depression, anxiety, and acculturation were examined.

To further ascertain their relationships, a forward multiple regression was calculated 
using acculturation as the criterion and anxiety and depression as predictor variables. Further analyses were conducted to explore the relationships of demographics and other variables.

\section{Assumptions and Limitations}

Several assumptions about the methodology and limitations were identified. The small size of the sample was the primary limitation of this study. Because only those who chose to participate were included in the study, the sample may have been skewed in some manner not detected by the investigator. The assumption that personal contacts were essential in order to gain access to mothers in the community was strongly supported by specific statements to that effect from all interpreters and key informants. Several key informants volunteered that the cultural norm was for privacy, and families were generally reluctant to share personal information with persons of the same ethnic group.

The refusal of a few mothers to participate after reading the consent form could have been related to the statement that the investigator was mandated to report child abuse. Given the history of inappropriate reports made to Child Protective Services in the Hmong and Vietnamese communities around issues of coining etc., one could expect some hesitancy related to inviting an unknown nurse into the home.

An assumption was made in that, by using identifiable religious groups, it could have been difficult to access various Hmong practitioners of traditional religions. Such an omission would have excluded an important group of Hmong participants. This assumption was not supported. Six Hmong participants did not claim affiliation with any church, and the husband of one of those participants was a shaman. It was difficult, however, to access significant numbers of the Vietnamese Buddhist community.

The inability of the investigator to speak the native language of the participant did slow the home visit. Interpreters were used when needed. The use of multiple interpreters in the Vietnamese population was a limitation. The investigator had to explain repeatedly the role of an interpreter to each new interpreter. Because the the data were not technical, the interpreters understood all the terms and the questions, thus making the translation of each question easier. The interpreters could have influenced the responses in some way not 
detected by the investigator. Translations of the consent could have introduced unintended meanings or biases. Every effort was made to standardize the visit and control the content, however it was not possible to predict or control every encounter.

It was impossible to determine the truthfulness of the responses. From past experiences with Vietnamese and Hmong populations, and from feedback given by the interpreters after leaving the home, all responses appeared authentic. If the participant did not wish to answer a question, she so indicated, as was done by five of the thirty participants for the Hopkins-25 item about sexuality. Three husbands participated in the interview, supplementing the response given by the participant, or at times disagreeing with her response. In those situations, validity could have possibly been compromised.

Instruments were chosen for compatibility of language and concepts. An assumption was made that the SL-ASIA and the HCSL-25 used language and constructs that were functionally equivalent in Vietnamese, Hmong and doninant cultures in the United States. Those particular tools had been developed by researchers who had an emic, or insider's, perspective and were selected for their cross-cultural authenticity and accuracy as demonstrated in previous studies by other researchers. It was possible for bias or misleading phrases to have been introduced during the translation process. If so, the quality of the data, and its validity and reliability could have been compromised in some way not detected.

In addition to a small sample size, there were other statistical limitations. With a Cohen's Kappa indicating that the proportion of agreement on maternal sensitivity between the experts and the investigator was only .52 and .40 , there could have been error in scoring of maternal sensitivity. Even if all the scores were inaccurate by one point, however, the trend would have remained the same. It is expected that the findings would not have been significantly different. The skewed distribution of the anxiety subscale could have influenced the results. When a statistical expert was consulted ( $R$. Algozzine, University of North Carolina at Charlotte, 3/31/98), he opined that the positive skew would not affect the results of correlations nor of the particular regressions calculated for the present study. 


\section{CHAPTER 4}

\section{Findings of the Study}

The purpose of chapter four is to describe relationships among maternal sensitivity, Posttraumatic Stress, anxiety, depression and acculturation in Vietnamese and Hmong mothers. First, the sample is described and differences between subgroups are detailed (Appendix J). Data about each research question is presented in the order in which it was asked. Data for the combined groups are presented; then, when warranted by statistical analyses, data for individual Vietnamese and Hmong groups are included. The Statistical Package for the Social Sciences 6.1 (SPSS) was used to analyze the data, explore relationships, and perform regression analyses to identify if there were predictive patterns among the variables.

\section{Description of the Sample}

Table 2 summarizes pertinent demographic characteristics. The sample was divided evenly between Vietnamese and Hmong with, 17 Vietnamese and 16 Hmong participants. One Vietnamese case was deleted because of incomplete information. That left 16 participants in each group for a total number of 32 participants. The ages of the mothers ranged from 17-43 years, with more than half of the sample between the ages of 25-35 years. One-quarter had lived in the United States less than five years, a second quarter between five and eight years, a third quarter between eight and thirteen years, with the final quarter having lived in the U.S. between 14-21 years. Religious affiliation varied; six were Roman Catholic, eight were Protestant, and three were Buddhist. Ten Hmong participants 
Table 2

Education, Occupation. and Religion of Participants by Ethnic Group

\begin{tabular}{|c|c|c|c|}
\hline & Vietnamese & Hmong & Total \\
\hline \multicolumn{4}{|l|}{ Education in Homeland } \\
\hline None & 0 & 15 & 15 \\
\hline$<12$ Years & 10 & 1 & 11 \\
\hline$>12$ Years & 6 & 0 & 6 \\
\hline \multicolumn{4}{|l|}{ Education in U. S. A. } \\
\hline None & 7 & 6 & 13 \\
\hline ESL Only & 4 & 4 & 4 \\
\hline High School & 0 & 5 & 5 \\
\hline College & 5 & $l$ & 6 \\
\hline \multicolumn{4}{|c|}{ Occupation in Homeland } \\
\hline Home/Intant & 2 & 9 & 11 \\
\hline Farm & 0 & 5 & 5 \\
\hline Student & 5 & 1 & 6 \\
\hline Other & 9 & 1 & 10 \\
\hline \multicolumn{4}{|l|}{ Occupation in U. S. A. } \\
\hline Home & 5 & 12 & 17 \\
\hline Student & 2 & 1 & 3 \\
\hline Other & 9 & 3 & 12 \\
\hline \multicolumn{4}{|l|}{ Religion } \\
\hline Catholic & 6 & 0 & 6 \\
\hline Protestant & 8 & 0 & 8 \\
\hline Latter Day Saints & 0 & 10 & 10 \\
\hline Buddhist & 3 & 0 & 3 \\
\hline Traditional & 0 & 6 & 6 \\
\hline
\end{tabular}


were Latter Day Saints, and six practiced Hmong traditional religion. The educational achievements of the women varied greatly. Six Hmong participants were unable to read any language and denied ever attending school. The remainder of the sample had been in school either in their home country or in the United States for varying lengths of time. Nine had attended only English as a Second Language (ESL) classes in theUnited States, but three were college graduates, and three others had completed community college in San Diego. In addition, one woman immigrated with a college degree, and two more came with the equivalent of a community college education. Seventeen of the sample did not work outside the home. Of the remaining sixteen women, three attended school, four worked in a factory, two worked in beauty salons, and two worked in the textile industry as seamstresses. Of the last four, one was a dental assistant, one a school aide, one a child development specialist, and one a sales manager in a store. The sample was almost evenly divided between those who used an interpreter (15 used interpreters) for the interview and those who spoke English.

Vietnamese and Hmong groups were compared only on the predictur and selected demographic variables with significant differences between the groups. T-tests (Appendix $J$ contïrmed significant differences in PTS $\underline{M}=1.33$ for Vietnamese; $\underline{M}=1.98$ for Hmong; $\underline{p}=.003)$, depression $(\underline{M}=1.34$ for Vietnamese; $\underline{M}=2.15$ for Hmong; $\underline{\underline{p}}=$ $.001)$, the number of pregnancies $\underline{M}=2.31$ for Vietnamese; $\underline{M}=5.69$ for Hmong; $\underline{p}=$ $.001)$ and the number of years in transition from the homeland to the United States $(\underline{M}=$ 0.31 tor Vietnamese; $\underline{M}=6.56$ for Hmong; $R=.001$ ). Based on the results of Bonferroni's $T$ for maternal sensitivity and the predictor variables, the level of significance $(\alpha)$ was set at .05 for all further statistical tests involving both combined and individual Vietnamese and Hmong groups.

\section{Research Question One}

To what extent do mothers in Vietnamese and Hmong populations experience posttraumatic stress (PTS)?

PTS was measured by the total score obtained from the HSCL-25 (Mollica, et al., 
1987). Anxiety and depression were each measured by subscales of HSCL-25. The number of participants, means, ranges of scores, and standard deviations were computed for the complete group, then for the Vietnamese and Hmong subgroups (Table 3). Two Hmong mothers chose not to complete the HSCL-25, thus the subgroups were unequal.

HSCL-25 and its subscales of depression and anxiety were highly correlated $(r=$ 96. $\mathrm{p}<.001$ for depression; $\underline{\mathrm{r}}=.86, \mathrm{p}<.001$ for anxiety) (Table 4). From these scores, it was concluded that the domains of anxiety and depression captured the essence of the stress experienced by this sample. In addition, depression accounted for most of the variance in PTS in both groups. Depression and anxiety were very bighly correlated in the Vietnamese group $(r=.95, \underline{p}<.001$ ) (Table 5), but not in the Hmong group $(r=.42, \mathfrak{p}=$ ns) (Table 6).

To more clearly grasp the relative contributions of depression and anxiety to PTS in each group, a forward multiple regression with PTS as the dependent variable, was performed and relationships between PTS, depression. and anxiety svaluated in the comoined grcup, then in the Vietnamese and Hmong groups (Table 7). In the combined group, depression accounted for $92 \%$ of the variance in PTS, with anxiety only contributing $7 \%$. In the Vietnamese, depression contributed $98 \%$ of the variance and anxiety only one percent. In contrast, depression contributed $80 \%$ of the variance, and anxiety contributed $19 \%$ in the Hmong group. Given these differences between the groups and the small sample size, depression and anxiety were substituted for PTS rather than simply added as two additional variables.

Means (Tabie 3) for the combined group of mothers were 1.51 on the anxiety scale, and 1.72 on the depression scale. With 1.75 accepted as the clinical cutorf for symptoms (Mollica et al, 1987; Mouanoutoua \& Brown, 1995; Nicholson, 1997), this group of mothers experienced nearly clinical levels of depression and anxiety. The mean for Vietnamese mothers was only in the very low symptomatic range -- 1.33 on the anxiety scale, and 1.34 on the depression scale. Means for Hmong mothers were much higher, in the clinical range, at 1.71 on the anxiety scale, and 2.15 on the depression scale (Figure 4). The range of scores was similar for both Vietnamese and Hmong mothers, with the 


\section{NOTE TO USERS}

\section{Page(s) missing in number only; text follows. Microfilmed as received.}

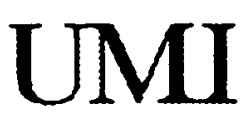


number of participants, means, ranges of scores, and standard deviations were computed for the complete group, then for the Vietnamese and Hmong subgroups (Table 3). Two Hmong mothers chose to not complete the HSCL-25, thus the subgroups were unequal.

HSCL-25 and its subscales of depression and anxiety were highly correlated $(r=$ $.96, \mathrm{R}<.001$ for depression; $\underline{r}=.86, \mathrm{R}<.001$ for anxiety) (Table 4). From these scores, it was concluded that the domains of anxiety and depression captured the essence of the stress experienced by this sample. In addition, depression accounted for most of the variance in PTS in both groups. Depression and anxiety were very highly correlated with each other in the Vietnamese group $(r=.95, p<.001$ ) (Table 5), but not in the Hmong group $(r=.42, \underline{p}=\underline{\mathrm{ns}})$ (Table 6$)$.

To more clearly grasp the relative contributions of depression and anxiety to PTS in each group, a forward multiple regression with PTS as the dependent variable, was performed and relationships between PTS, depression, and anxiety evaluated in the combined group, then in the Vietnamese and Hmong groups (Table 7). In the combined group, depression accounted for $92 \%$ of the variance in PTS, with anxiety only contributing $7 \%$. In the Vietnamese, depression contributed $98 \%$ of the variance and anxiety only one percent. In contrast, depression contributed $80 \%$ of the variance, and anxiety contributed $19 \%$ in the Hmong group. Given these differences between the groups and the small sample size, depression and anxiety were substituted for PTS rather than simply added as two additional variables.

Means (Table 3) for the combined group of mothers were 1.51 on the anxiety scale, and 1.72 on the depression scale. With 1.75 accepted as the clinical cutoff for symptoms (Mollica et al, 1987; Mouanoutoua \& Brown, 1995; Nicholson, 1997), this group of mothers experienced nearly clinical levels of depression and anxiety. The mean for Vietnamese mothers was only in the very low symptomatic range - 1.33 on the anxiety scale, and 1.34 on the depression scale. Means for Hmong mothers were much higher, in the clinical range, at 1.71 on the anxiety scale, and 2.15 on the depression scale (Figure 4). The range of scores was similar for both Vietnamese and Hmong mothers, with the 
Table 3

Mean. Range, and Standard Deviation of PTS, Anxiety, and Depression

\begin{tabular}{|c|c|c|c|c|c|c|c|c|c|c|c|c|c|c|c|}
\hline \multirow[b]{2}{*}{ Variable } & \multicolumn{5}{|c|}{ Combined Groups } & \multicolumn{5}{|c|}{ Vietnamese } & \multicolumn{5}{|c|}{ Hmong } \\
\hline & $\mathbf{N}$ & $\mathbf{M}$ & Min & $\operatorname{Max}$ & $\mathrm{SD}$ & $\mathbf{n}$ & $\mathbf{M}$ & Min & $\operatorname{Max}$ & $\underline{S D}$ & $\mathbf{n}$ & $\mathbf{M}$ & Min & $\operatorname{Max}$ & $\underline{\mathrm{SD}}$ \\
\hline PTS & 30 & 1.63 & 1.00 & 3.20 & .63 & 16 & 1.33 & 1.00 & 3.20 & .54 & 16 & 1.98 & 1.00 & 2.80 & .55 \\
\hline Anxicty & 30 & 1.51 & 1.00 & 3.10 & .62 & 16 & 1.33 & 1.00 & 3.10 & .53 & 14 & 1.71 & 1.00 & 2.90 & .66 \\
\hline Depression & 30 & 1.72 & 1.00 & 3.30 & .72 & 16 & 1.34 & 1.00 & 3.30 & .55 & 14 & 2.15 & 1.00 & 3.10 & .64 \\
\hline
\end{tabular}

Note, PTS $=$ Posttraumatic Stress 
Table 4

Correlation Coefficients ( $r$ ) of Predictor and Demographic Variables for Combined Groups

\begin{tabular}{|c|c|c|c|c|c|c|c|c|c|}
\hline Variables & Sensitivity & PTS & Anxiety & Depress & Accult & \# Hub Fam+ & \# Pregnant & Age & \# Yrs Trans \\
\hline Sensitivity & 1.00 & .07 & -.08 & .13 & -.26 & $-.31 *$ & .22 & .26 & $.42^{* *}$ \\
\hline PIS & & 1.00 & $.86 * * *$ & $.96 * * *$ & $.48^{* *}$ & -.15 & $.69 * * *$ & .30 & $.34^{*}$ \\
\hline Anxiety & & & 1.00 & $.69 * * *$ & $-.57 * *$ & -.26 & $.43^{* *}$ & .21 & .21 \\
\hline Depression & & & & 1.00 & $-.40 *$ & -.07 & $.76^{* * *}$ & .33 & $.36 *$ \\
\hline Acculturation & & & & & 1.00 & $.60 * * *$ & $-.46^{* *}$ & $-.53 * *$ & -.23 \\
\hline \# Husb Fam+ & & & & & & 1.00 & -.20 & -.46 & .02 \\
\hline \# Pregnant & & & & & & & 1.00 & $.51^{* *}$ & $.35^{*}$ \\
\hline Age & & & & & & & & 1.00 & -.13 \\
\hline \# Yrs in Trans & & & & & & & & & 1.00 \\
\hline
\end{tabular}

Note. PTS = Postraumatic Stress; $+=$ Number of Husband's Family in the USA; \# Yrs Trans = Number of Years in Transition.

$*=\mathrm{p}<0.05 ; * *=\mathrm{p}<0.01 ; * * *=\mathrm{p}<.001,(1$-tailed) 
Table 5

Correlation Coefficients (r) of Predictor and Demographic Variables for Vietnamese Group

\begin{tabular}{|c|c|c|c|c|c|c|c|c|c|}
\hline Variables & Sensitivity & PTS & Anxiety & Depress & Accult & \# Hub Fam+ & \# Pregnant & Age & \# Yrs Trans \\
\hline Sensitivity & 1.00 & -.39 & -.36 & -.40 & -.07 & -.24 & -.22 & .22 & -.16 \\
\hline PIS & & 1.00 & $.98^{* * *}$ & $.99 * * *$ & $-.45^{*}$ & $-.44 *$ & $.75^{* * *}$ & $.58^{* *}$ & -.32 \\
\hline Anxiety & & & 1.00 & $.95 * * *$ & -.49 & $-.48^{*}$ & $.72^{* *}$ & $.59 * *$ & -.36 \\
\hline Depression & & & & 1.00 & $-.43 *$ & -.41 & $.73 * *$ & $.60^{* *}$ & .30 \\
\hline Acculturation & & & & & 1.00 & $.56^{*}$ & -.19 & $-.48^{*}$ & $.54^{*}$ \\
\hline \# Hub Fam+ & & & & & & 1.00 & $-.53^{*}$ & $-.53^{*}$ & .19 \\
\hline \# Pregnant & & & & & & & 1.00 & $.66^{* *}$ & -.06 \\
\hline Age & & & & & & & & 1.00 & -.37 \\
\hline \# Yrs Trans & & & & & & & & & 1.00 \\
\hline
\end{tabular}

Note. PIS = Postraumatic Stress; + = Number of Husband's Family in the USA; \# Yrs Trans = Number of Years in Transition.

$*=\mathrm{p}<0.05 ; * *=\mathrm{p}<0.01 ; * * *=\mathrm{p}<.001$, (1-tailed) 
Table 6

Correlation Coefficients ( $r$ ) of Predictor and Demographic Variables for Hmong Group

\begin{tabular}{|c|c|c|c|c|c|c|c|c|c|}
\hline Variables & Sensitivity & PTS & Anxiety & Depress & Accult & \# Hub Fam+ & \# Pregnant & Age & \# Yrs Trans \\
\hline Sensitivity & 1.00 & .32 & .03 & .43 & -.36 & $-.45^{*}$ & .31 & .37 & $.57^{*}$ \\
\hline Pls & & 1.00 & $.77 * *$ & $.90 * * *$ & $-.63 * *$ & -.20 & $.53 *$ & $.53^{*}$ & .05 \\
\hline Anxiety & & & 1.00 & .42 & $-.65^{* *}$ & -.27 & .19 & .18 & .08 \\
\hline Depression & & & & 1.00 & $-.48^{*}$ & -.11 & $.65^{* *}$ & $.64^{* *}$ & .03 \\
\hline Acculturation & & & & & 1.00 & $.70^{* *}$ & $-.59 * *$ & $-.62 * *$ & -.28 \\
\hline \# Hub Fam+ & & & & & & 1.00 & -.39 & -.40 & -.20 \\
\hline \# Pregnant & & & & & & & 1.00 & $.90 * * *$ & -.03 \\
\hline Age & & & & & & & & 1.00 & .05 \\
\hline \# Yrs Trans & & & & & & & & & 1.00 \\
\hline
\end{tabular}

Note. PIS = Postraumatic Stress; $+=$ Number of Husband's Family in the USA; \# Yrs Trans = Number of Years in Transition

$*=\mathrm{p}<0.05 ; * *=\mathrm{p}<0.01 ; * * *=\mathrm{p}<.001,(1$-tailed $)$ 
Table 7

Relationships of PTS, Depression, and Anxiety

\begin{tabular}{|c|c|c|c|c|c|c|c|c|c|c|c|c|c|c|c|}
\hline \multirow[b]{2}{*}{ Variables } & \multicolumn{5}{|c|}{ Combined } & \multicolumn{5}{|c|}{ Vietnamese } & \multicolumn{5}{|c|}{ Hmong } \\
\hline & Source & ss & $\mathrm{df}$ & MS & $\mathrm{F}$ & Source & ss & df & MS & $\mathrm{F}$ & Source & ss & df & MS & $\mathbf{F}$ \\
\hline Depression & Regress & 10.46 & 1 & 10.46 & 319.10 & Regress & 4.22 & 1 & 4.22 & 654.61 & Regress & 3.21 & 1 & 3.21 & 52.71 \\
\hline Anxiety & Regress & 11.36 & 2 & 5.68 & 6045.04 & Regress & 4.30 & 2 & 2.15 & 1866.57 & Regress & 3.92 & 2 & 1.96 & 2137.55 \\
\hline
\end{tabular}


Figure 4

Comparison of Depression and Anxiety by Ethnicity

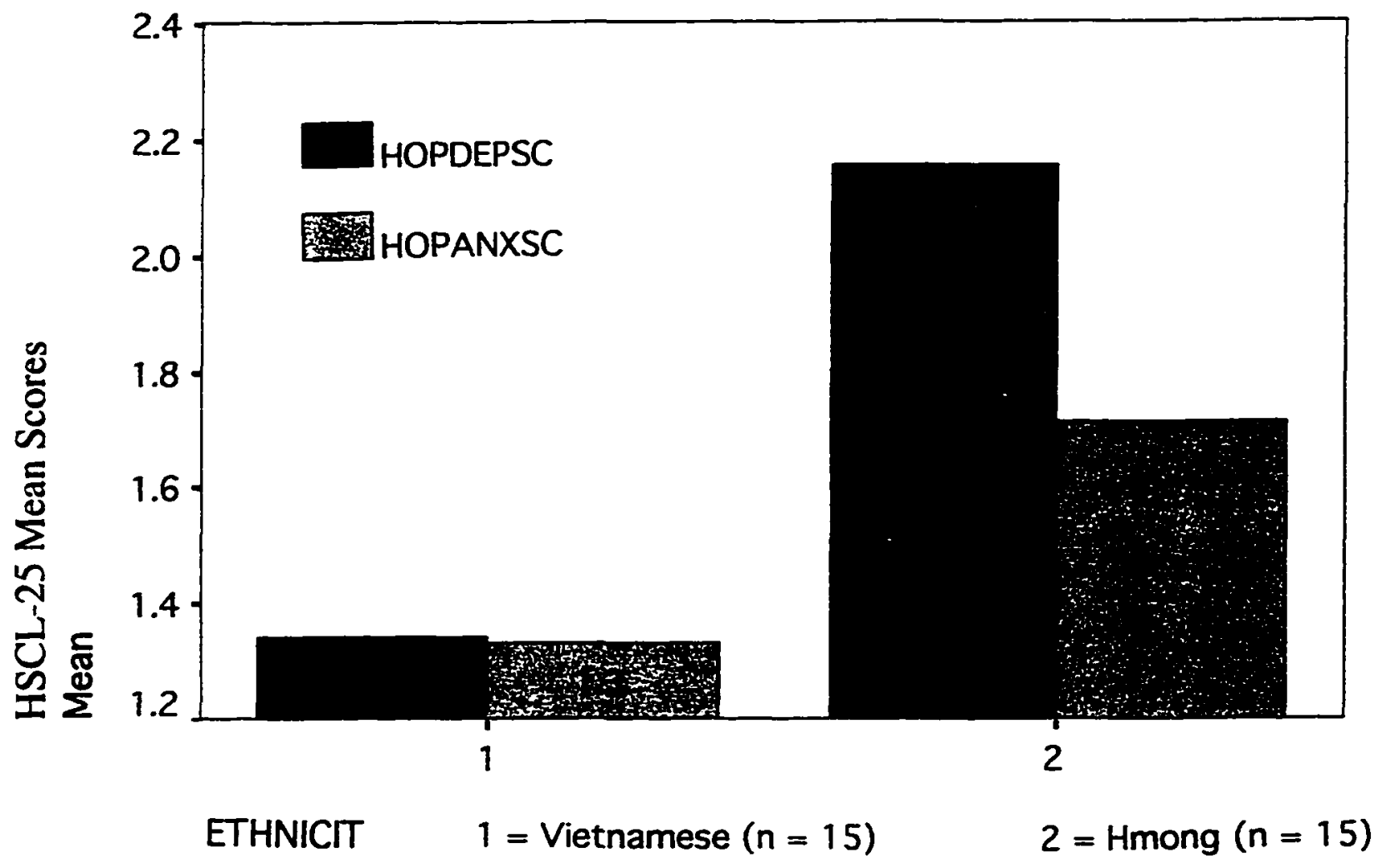

widest range for the anxiety score actually occurring in the Vietnamese group (Table 3 ). Forty-three percent of the combined group of mothers reported clinical levels of depression or anxiety. When individual groups were examined, $78.6 \%$ of Hmong mothers were depressed or anxious while only $12.5 \%$ of the Vietnamese sample were clinically depressed or anxious.

\section{Research Question Two}

To what extent does PTS, anxiety, or depression decrease maternal sensitivity in Vietnamese and Hmong mothers?

Based on the findings in Question One, PTS was replaced by anxiety and depression. Since there was no difference (Appendix $\mathrm{J}$ ) between the groups in degree of maternal sensitivity $(\underline{M}=7.81$ for Vietnamese; $\underline{M}=8.13$ for Hmong) or anxiety $(\underline{M}=$ 
1.33 for Vietnamese; $\underline{M}=1.71$ for Hmong), anxiety was analyzed for only the combined group. A significant difference (Appendix $)$ did exist for depression $(\mathbf{M}=1.34$ for Vietnamese; $\underline{M}=2.15$ for Hmong; $\underline{\not}=.001$ ), so depression was analyzed for both Vietnamese and Hmong groups.

Higher levels of anxiety did not decrease maternal sensitivity in the combined group of Vietnamese and Hmong mothers who participated in this study $(r=-.08, \underline{p}=\underline{n s})$ (Table 4). Because there was no difference between the groups (Appendix J), no individual group analyses were performed.

There was a trend for increased depression to decrease matemal sensitivity only in the Vietnamese group. A moderate negative correlation approaching significance $(r=-.40 \underline{p}$ $<.1)$ was found between depression and maternal sensitivity in the Vietnamese group (Table 5). Nearly the identical correlation was noted in the Hmong group (Table 6), but in the opposite direction $(r=.43 \mathrm{R}<.1)$. Thus the more depression Vietnamese mothers reported, the less sensitive they were toward their infants, and the more depression Hmong mothers reported, the more sensitive they were to their infants. To further explore the trend between depression and maternal sensitivity, a multiple regression was performed with a forward entry for matemal sensitivity, anxiety and depression, with ethnicity as a dummy variable. Even with the PIN level set at .10, depression was not accepted into the equation. It was concluded that the association was mostly by chance.

\section{Research Question Three}

To what extent does acculturation decrease maternal sensitivity in

\section{Vietnamese and Hmong mothers?}

The more the mothers were acculturated, the less sensitive to their infants they tended to be $(r=-.26 \mathrm{p}<.10)$ (Table 4). It was established earlier that there was no difference between the groups in levels of maternal sensitivity. In addition there also was no difference between the groups (Appendix $J$ ) in the degree of acculturation $M=2.14$ for Vietnamese; $\underline{M}=2.04$ for Hmong), so Vietnamese and Hmong mothers were not subdivided for this analysis. 


\section{Research Question Four}

\section{Among Vietnamese and Hmong mothers, is there a relationship between PTS, or its components of depression and anxiety, and acculturation?}

Coefficients for acculturation, depression, and anxiety (Tables $4,5, \& 6$ ) were examined for possible relationships. Because there was no difference between Hmong and Vietnamese mothers in the degree of acculturation, $\underline{M}=2.14$ for Vietnamese; $\underline{M}=2.04$ for Hmong) or anxiety $(\underline{M}=1.33$ for Vietnamese; $\underline{M}=1.71$ for Hmong), the groups were not examined separately for that relationship. Because Vietnamese and Hmong mothers did report a significant difference in the degree of depression experienced $\underline{M}=1.34$ for Vietnamese; $\underline{M}=2.15$ for Hmong; $\underline{p}=.001$ ), data were analyzed separately for acculturation and depression. More anxious mothers were less acculturated $(\underline{r}=-.57, \mathbb{R}<$ .01) (Table 4). The same pattem was found for depression in both Vietnamese $(\underline{r}=-.43, \underline{p}$ $<.05)$ (Table 5) and Hmong $(r=-.48, \mathrm{p}<.05)$ (Table 6) mothers.

To identify if there was any predictive pattern to the relationships of acculturation, anxiety, and depression, a multiple regression with forward entry was performed for the combined group, then for individual groups (Table 8). Acculturation was entered as the dependent variable. In the combined group, only anxiety emerged as predictive of acculturation, accounting for $30 \%$ of the variance. In the Vietnamese group, neither anxiety or depression was predictive of acculturation; however, in the Hmong group, anxiety was predictive of $38 \%$ of th variance in acculturation. When the PIN was changed from .05 to .10 anxiety also emerged as a predictor variable accounting for $18 \%$ of the variance in acculturation in the Vietnamese group.

\section{Other Findings}

Multiple regression was performed to determine if there were any predictive relationships between any of the variables in the model. When acculturation, anxiety, and depression were entered at once, they only accounted for $12 \%$ of the variance in maternal 
Table 8

Relationships of Acculturation, Depression, and Anxiety

\begin{tabular}{|c|c|c|c|c|c|c|c|c|c|c|c|c|c|c|c|}
\hline \multirow[b]{2}{*}{ Variables } & \multicolumn{5}{|c|}{ Combined } & \multicolumn{5}{|c|}{ Vietnamese } & \multicolumn{5}{|c|}{ Hmong } \\
\hline & Source & ss & df & MS & $\mathrm{F}$ & Source & ss & $\mathrm{df}$ & MS & $F$ & Source & ss & $\mathrm{df}$ & MS & $\mathbf{F}$ \\
\hline Anxiety & Regress & 2.09 & 1 & .16 & $13.49 * * *$ & Regress & .49 & 1 & .49 & $4.33 *$ & Regress & 1.85 & 1 & 1.85 & $8.96^{* *}$ \\
\hline
\end{tabular}

Note. PIN set at .10; * $\mathfrak{p}<.10 ; \quad * * \mathfrak{p}<.05 ; * * * \mathfrak{p}<.01$ 
sensitivity in the total sample. In the Vietnamese group, the variables in the model only accounted for $5 \%$ of the variance, and in the Hmong group only $14 \%$. When a forward entry was used, none of the variables had any predictive relationship to maternal sensitivity.

Exploratory correlation analyses were conducted to identify if there were any other trends in the data (Tables $4,5, \& 6$ ). The data were first examined for correlations between maternal sensitivity and several demographic variables. Subsequently, correlations between selected demographic variables, PTS, anxiety, depression, and acculturation were examined. Where t-tests confirmed significant differences (Appendix J) between the groups, correlations among variables are presented for both groups.

Two demographic variables significantly correlated with maternal sensitivity (Table 4); the number of years in transition from the homeland to the USA $(r=.42, p<.01)$, and the number of the husband's family in the United States $(r=-.31, p=<.05)$. Only the group means for the number of years in transition from the homeland to the United States were different (Appendix $\mathrm{J}$ ) between the Vietnamese and the Hmong $(\underline{M}=.31$ for Vietnamese; $\underline{M}=6.6$ for Hmong; $\underline{p}=.001$ ). When individual groups were examined, it was the more sensitive Hmong mothers who had spent more time in transition $(r=.56, \underline{p}=$ $.01)$. No association was found for the number of years in transition and maternal sensitivity in the Vietnamese group. Greater numbers of the husband's family members living in the United States was associated with less maternal sensitivity $(r=-.31, \mathrm{p}=<$ $.05)$.

When correlations among anxiety, depression, acculturation, and selected demographic variables were analyzed (Tables $4,5, \& 6$ ), a number of associations were noted. Older mothers were less acculturated $(r=-.53, \underline{p}<.01)$ and more depressed $(r=$ $.59, \mathrm{p}<.01$ for Vietnamese; $\underline{r}=.64, \mathrm{p}<.01$ for Hmong) and had borne more children $(r=$ $.66, \underline{p}<.01$ for Vietnamese; $\underline{r}=.90, \mathrm{p}<.001$ for Hmong). The more members of the husband's family who lived in the United States, the greater the level of acculturation $(\underline{r}=$ $.60, \mathrm{p}<.001)$. Less acculturation was associated with more pregnancies only in the Hmong group $(r=.59, \mathrm{p}<.01)$.

Perhaps one of the most startling findings was the high association of greater 
numbers of pregnancies and higher levels of depression $(r=.73, p<.01$ for Vietnamese; $\underline{r}$ $=.65, \mathrm{p}<.01$ for Hmong). In the Vietnamese group, more pregnancies was also associated with higher anxiety $(r=.72, \mathrm{p}<.01)$, but there was no relationship between acculturation and number of pregnancies $(r=-.19, \mathrm{p}=.24)$. On the other hand, more acculturated Hmong mothers had fewer pregnancies $(r=-.59, \underline{p}<.01)$. More pregnancies were associated with fewer of the husband's family in the United States $(r=-.53, \underline{p}<.05$ for Vietnamese; $\underline{r}=-.39, \mathrm{R}<.10$ for Hmong), especially among the Hmong.

To explore if there were any possible predictive relationships between the demographic variables and maternal sensitivity, a multiple regression with a forward entry was performed. Maternal sensitivity was entered as the dependent variable, and the number of years in transition, the number of husband's family members in the United States, and age were entered as predictor variables. Only the number of years in transition predicted $15 \%$ of the variance in matemal sensitivity in the combined group, nothing predicted maternal sensitivity in the Vietnamese, but the number of years in transition predicted $27 \%$ of the variance in the Hmong group. When the same three variables were entered again with the PIN set at .10, the combination of number of years in transition and age together accounted for $23 \%$ of the variance in maternal sensitivity. A different picture emerged when each group was considered individually. No variables were accepted into the equation for the Vietnamese group. For the Hmong, the number of years in transition accounted for $27 \%$ of the variance. Age did not contribute to the variance in either the Hmong or the Vietnamese groups. (Table 9). Due to the small size of the sample and the number of variables, the demographic variables were not entered into a regression equation with the predictor variables.

\section{Summary}

In summary, this population of Vietnamese and Hmong women were found to experience PTS, and specifically the domains of depression and anxiety. Only very slightly decreased maternal sensitivity was found when anxiety, depression and acculturation were increased. The combination of the predictor variables in the model together only accounted for $5 \%$ of the variance in maternal sensitivity in the Vietnamese group and $14 \%$ of the 
Table 9

Relationships of Maternal Sensitivity, Age, Number of Years in Transition, and Number of Husband's Family in the United States

\begin{tabular}{|c|c|c|c|c|c|c|c|c|c|c|c|c|c|c|}
\hline \multirow[b]{2}{*}{ Variables } & \multicolumn{5}{|c|}{ Combined } & \multicolumn{4}{|c|}{ Vietnamese } & \multicolumn{5}{|c|}{ Hmong } \\
\hline & Source & ss & $\mathrm{df}$ & MS & $F$ & Source & $\mathrm{df}$ & MS & $F$ & Source & ss & $\mathrm{df}$ & MS & $\mathrm{F}$ \\
\hline \# Years+ & Regress & 5.21 & 1 & 5.21 & $6.36^{* *}$ & \multicolumn{4}{|c|}{ No variables entered the equation } & Regress & 5.02 & 1 & 5.02 & $6.54^{* *}$ \\
\hline Age & Regress & 8.21 & 2 & 4.10 & $5.54 * * *$ & \multicolumn{4}{|c|}{ No variables entered the equation } & \multicolumn{5}{|c|}{ No variables entered the equation } \\
\hline
\end{tabular}

Note. $+=$ Number of Years in Transition; PIN set at .10; ${ }^{*}=\underline{p}<.10 ; * *=\underline{p}<.05 ; * * *=p<.001 ;$ 
variance in the Hmong. When acculturation was increased, so were anxiety and depression, with anxiety and acculturation showing the strongest relationship.

Among the demographic variables, an increased number of years spent in transit from the homeland to the United States was associated with greater maternal sensitivity. This was particularly true of the Hmong group. In the combined group, the greater number of the husband's family who lived in the United States, the lower was the mother's sensitivity.

Finally, the more pregnancies reported by older Vietnamese mothers, the greater their degree of depression $(r=73, p<.01)$ and anxiety $(r=.72, \mathrm{R}<.01)$. Hmong mothers who reported more pregnancies also reported higher levels of depression $(r=.65, \underline{p}<$ .01 ). Because the sample size was small, these three demographic variables (age, number of years spent in transit, and number of husband's family in the United States) were substituted for the predictor variables. They accounted for none of the variance in maternal sensitivity in the Vietnamese group, but did account for $27 \%$ of the variance in the Hmong group. 


\section{CHAPTER 5}

\section{Discussion of the Findings}

The purpose of chapter five is to discuss the findings of the study in view of the theoretical relationships that were predicted. The initial discussion focuses on the levels of PTS, anxiety and depression found in this sample of mothers. Secondly, the significance of relationships among maternal sensitivity, PTS, anxiety, depression, and acculturation that were suggested by the data are explored. Third, a discussion of other findings from this study are discussed. Finally, conclusions and recommendations for practice and future research are offered. Although no qualitative data were collected in this study, anecdotal comments from the participants offer background information and possible explanations for findings. Because the investigator collected data from all participants, some of the comments are interjected where deemed appropriate.

\section{Overview of Findings}

\section{Results}

This study confirmed the presence of increased stress in mothers, but the theoretical relationships between PTS, anxiety, depression, acculturation, and maternal sensitivity (Figure 2) were not supported by the findings. The correlation coefficients ( $\mathrm{r}$ ) were added to the model (Figure 5). Anxiety and depression were shown to have very weak and nonsignificant relationships to maternal sensitivity. There was a nonsignificant trend for maternal sensitivity to decrease when participants were more acculturated.

Anxiety and PTS, as well as depression and PTS, were highly correlated (Figure 5), but anxiety and depression were only moderately correlated. Acculturation, anxiety, and depression were positively associated. The only variables that were correlated with 
Figure 5

\section{Specified Relationships of Predictor Variables to Maternal Sensitivity}

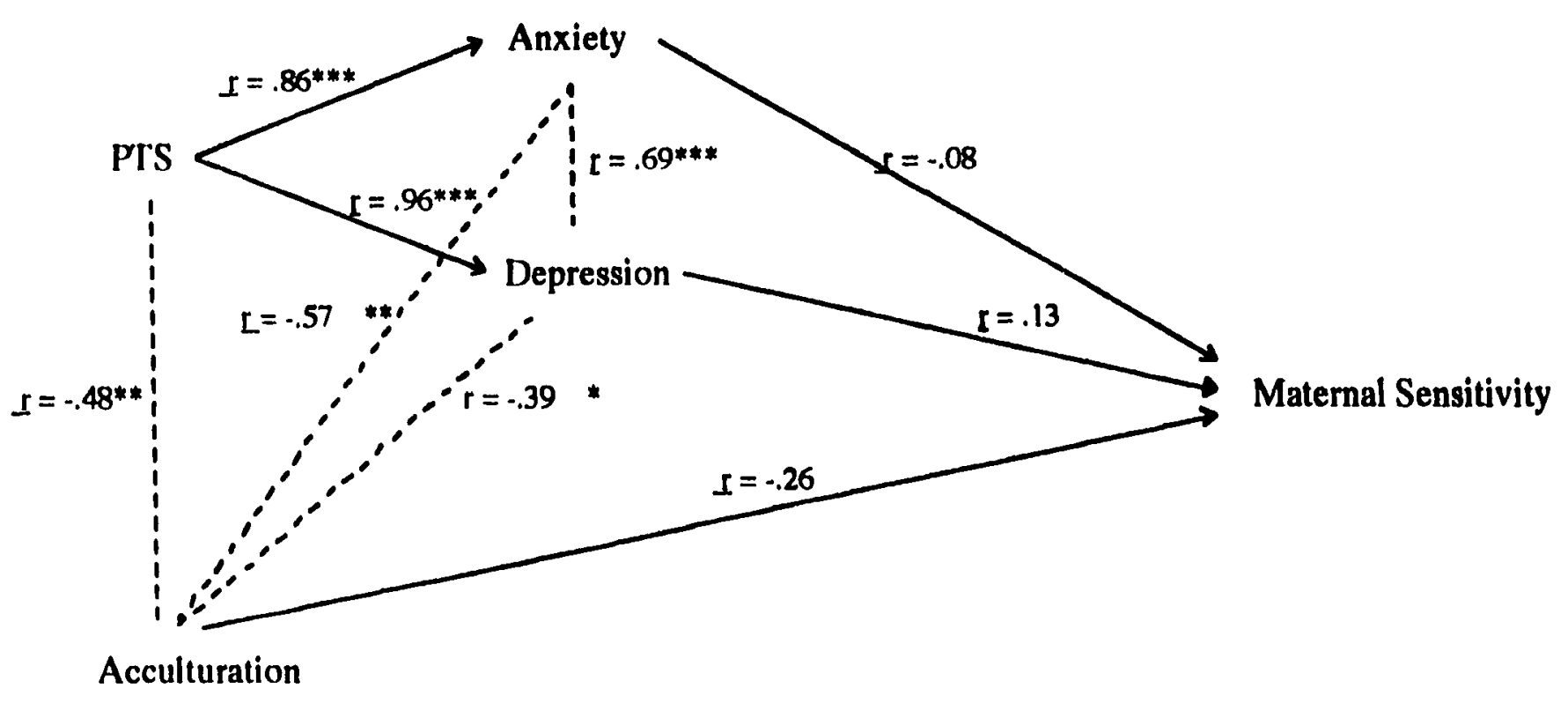

Note. PTS $=$ Posttraumatic Stress; $\underline{r}=$ correlation coefficient (one-tailed); ${ }^{*} \mathrm{p}<.05 ;{ }^{* *} \mathrm{p}<.01 ;{ }^{* * *} \mathrm{R}<.001$ 
maternal sensitivity were the number of years in transition and the number of the husband's family in the United States. The data suggested that the proposed contextual determinants of parenting in immigrant populations (premigration experiences, post-migration experiences, the mother's ethnicity, and the sociopolitical environment) influenced the meaning and expectations of the maternal role within a particular family system (Figure 1; Table 1); however, how they are associated to maternal sensitivity remains unclear. Methodology

Use of the interview format for data collection proved to be an effective mode. The use of instruments in the language of the participant was helpful. Different interpreters had the same words on a page, thus potential for bias with each new interpreter, or each family, was diminished.

Use of pretranslated materials helped maintain consistency throughout the datacollection process. Validation of the translation of the SL-ASIA with another set of translators would confirm conceptual equivalency. Use of a single interpreter for both groups would have been preferable, but that was not possible.

\section{Research Question One}

To what extent do mothers in Vietnamese and Hnong populations experience posttraumatic stress (PTS)?

In this sample, the two domains of anxiety and depression accounted for $99 \%$ of PTS, thus the assumption was made that anxiety and depression could conceptually substitute for PTS. As described in the methodology, when the HSCL, and subsequently the HSCL-25, was constructed as a screening tool for PTSD, it was the combination of depression and anxiety that were considered to be the essence of that medical diagnosis (Derogates, et al, 1974; Mollica, Wyshak, de Marneffe, et al., 1987; Winokur et al, 1984). Now the HSCL-25 is used by the medical profession to identify PTSD (as defined by the DSM-IV), anxiety, and depression, and it is used by professionals in other health care disciplines to identity general levels of stress, depression, and anxiety. Researchers have used depression and anxiety to represent stress (Nicholson, 1997; Yin \& Akutsu, 1997). Since depression and anxiety together appeared synonymous with PTS, the investigator 
decided to substitute those two variables for PTS. In addition, anxiety and depression apparently captured slightly different phenomena in this study, so investigation of their differences was desirable. With a small sample size, it was imperative not to increase the number of predictor variables beyond three. With three predictor variables, a minimum of ten participants per variable was maintained.

Consistent with findings from studies in the general population of Southeast Asians, and as measured by the HSCL-25, the combined groups of mothers in this study experienced near-clinical levels of anxiety (1.51) and depression (1.72). Vietnamese and Hmong mothers differed dramatically in the degree of both anxiety and depression they experienced. Vietnamese mothers scored 1.33 on the Anxiety Scale, and 1.34 on the Depression Scale, while Hmong mothers scored 1.71 on the Anxiety Scale and 2.15 on the Depression Scale. The magnitude of those differences was illustrated by the difference in the percentage of each group that reported clinical levels of anxiety or depression (12.5\% for Vietnamese; $78.6 \%$ fo: Hmong) (Figure 4) and by two questions on the HSCL-25. Eleven out of sixteen Hmong mothers reported feelings of hopelessness during the week prior to the interview, contrasted with three out of seventeen Vietnamese mothers reporting such feelings. Eight out of sixteen, or half of the sample of Hmong mothers, reported they had experienced thoughts of ending their life within the last week, while only three of the seventeen Vietnamese mothers reported the same thoughts within the week just prior to the interview.

When these results were compared with other studies that used the HSCL-25 to measure anxiety or depression, nearly identical results were found. Nicholson (1997) used the HSCL-25 and found anxiety scores to be 1.67 and depression scores to be 1.74 in her combined sample of four Southeast Asian refugee groups. Hinton (1997) reported a depression score of 1.31 and and anxiety score of 1.36 in his sample of Vietnamese. No studies were located that actually gave HSCL-25 scores of Hmong participants, so comparison of their scores with other studies was difficult.

Studies about PTS, anxiety, and depression in the Hmong population have generally focused on diagnostic and intervention strategies and have used tools that directly related to the DSM-III or DSM-IV. Chung and Kagawa-Singer (1993), Kroll, et al, 
(1989), Mollica, et al.(1990), and Yin. Akutsu, Zhang et al. (1997) all reported considerably higher levels of PTS. anxiety, and depression among the Hmong than the Vietnamese. Reasons for such a wide variation in scores have been generally related to the differences in their history during and subsequent to the Vietnam war and to experiences during resettlement. No references were found that addressed the role religious beliefs or woridview could have contributed to the differences in depression and anxiety between Vietnamese and Hmong groups. If such a relationship existed, it would be more likely to be observed between Buddhist Vietnamese and Hmong. As noted earlier, access to the Vietnamese Buddhist population in the current study was severely restricted.

Chung and Kagawa-Singer (1993), Nicholson (1997), and Yin, et al. (1997) all related the number of traumatic events experienced or witnessed in the homeland, and the number of post migration negative events, directly to anxiety and depression. In this study, questions were not asked about the number of traumatic events experienced or witnessed in the homeland. History verifies that indeed, as a group, the Hmong did experience many more traumatic events than the Vietnamese. After the Vietnam war, the general Vietnamese population was not targeted for systemic extermination as had been the Hmong. Given the historically large family size and extended family/clan system among the Hmong (Barney, 1979; Quincy, 1995), a finding of no statistical difference in the number of family members in either the homeland $(\underline{M}=9$ for Vietnamese; $\underline{M}=5$ for Hmong; $p=.258$ ) or the United States $\underline{M}=10$ for Vietnamese; $\underline{M}=14$ for Hmong; $\underline{p}=.141$ ) could represent a massive annihilation of family members among the Hmong people. Comments from Hmong participants supported such a view. Five Hmong respondents stated there were no family members of either the mother or the father in Laos, while only one Vietnamese mother reported no family in Vietnam. One Hmong respondent even stated that her husband had no family anywhere. He was the sole survivor of all of his family.

The verbal reports given to the investigator of hardships since arrival were consistent with reports by other authors (Tran, 1993; Yin \& Akutsu, 1997; Westermeyer, 1986). No questions were asked to this sample of women about their income or their welfare status, due to the intrusive nature of the questions; however, the contrast in housing between the two groups was stark, and provided some insight into income levels 
of both groups. Most of the Vietnamese had been in the United States six and a half years, had on average, two children and lived in unsubsidized housing, while the majority of the Hmong had lived in the United States an average of eleven years, had five to six children, and lived in smaller subsidized housing units provided for families receiving Aid to Families with Dependent Children (AFDC). Household furnishings among the Hmong were generally sparse, spartan, and worn. In contrast, most Vietnamese homes displayed well-used, but modern and comfortable furnishings. Such observations were consistent with Chung and Bemak's (1996) findings that correlated welfare dependency with psychological distress.

Nicholson (1997) reported that perceived health status predicted PTS, anxiety, and depression; the female gender predicted anxiety; and the level of income predicted depression. Since the present study used a self-reporting tool to identify stress, depression and anxiety, participants who scored higher on the HSCL-25 could also have been indicaling a perceived decreased health status. Indeed, three of the women who scored highest on the depression and anxiety scales did inform the investigator of significant health problems.

A sense of coherence does seem to explain the differential findings in depression and anxiety found between Vietnamese and Hmong mothers in this study. Vietnamese mothers had fewer children, had at least finished high school or its equivalent, or were attending school. While many referred to the relatively low status of wives in comparison to their husbands and to their husband's family, those comments were usually quickly followed by a description of how they managed to do what they perceived was needed for their own and their family's well-being. Descriptions of life in Vietnam were vivid and used to explain how they provided a base for functioning in the United States. References to French influences on their culture were often cited. The Vietnamese mothers who were more traditional in their family role and expectations reported depression scores much closer to those found in the Hmong group.

Many Hmong participants volunteered that it was impossible for them to return to Laos. Their land was gone, their family was gone, or most of them lived in the United States, and their children preferred to be with their friends in the "American" culture. 
Hmong women seemed resigned to having many children because it was their husband's desire. Many articulated a sense of alienation from the surrounding culture. An inability to speak English, read or write were some of the things they identified as separating Hmong women from the surrounding society. To a greater extent than the Vietnamese parents, Hmong parents verbalized concerns that their children were forgetting Hmong history and Hmong culture. Given the Hmong history of independence and maintenance of Hmong traditions in the face of great oppression from a variety of surrounding societies (Quincy, 1995), for this first generation to cling strongly to traditional ways is not surprising. Both men and women seemed to be searching for ways to make sense of their current life, their history, and their future as a distinct Hmong people, in other words, for a sense of coherence. While a sense of coherence could explain the presence of much higher rates of anxiety and depression among Hmong mothers, it does not explain why those very same mothers were exquisitely sensitive to their infants. Perhaps the infant who represents continuation of the family and clan, and fulfillment of a primary duty as a wife, provides meaning and even a sense of coherence to a mother. Hopefully future research will illuminate protective factors that operate on the infant's benefit.

\section{Research Question Two}

To what extent does PTS, anxiety, or depression decrease maternal sensitivity in Vietnamese and Hmong mothers?

For reasons stated earlier, only data about maternal sensitivity in the presence of anxiety and depression were examined. In spite of elevated anxiety and depression levels, maternal sensitivity remained dramatically higher than what has been reported in the literature for mothers from primarily European-American backgrounds. Vietnamese and Hmong mothers scores were not significantly different (Appendix J). With a combined group mean score of 7.97 , maternal sensitivity among mothers in this study was considerably higher than found in primarily European-Americans who have lived in the United States for generations. In the general population, the mean score for maternal sensitivity has been reported at 6.6 (Heermann et al., 1994). In the study that compared European American and Hmong mothers, in Minneapolis, MN, European-Americans only 
scored 5.44 whereas the mean score for Hmong mothers was 8.64 (Oberg, et al., 1986). There was a nonsignificant trend in the Vietnamese group for higher depression levels to be associated with lower matemal sensitivity, but the exact opposite trend occurred in the Hmong group. However, with only three mothers out of 32 scoring a 6.0 for maternal sensitivity and no mothers scoring below 6.0, that trend is inconclusive. Further investigation is needed before any conclusions can be formulated.

Several possibilities could account for the high degree of maternal sensitivity in this group of Vietnamese and Hmong mothers. Muret-Wagstaff and Moore (1989) hypothesized that the marked difference in maternal sensitivity between Hmong and European-American mothers was due to their history of high infant mortality rates. As a result, there was a need for much higher maternal sensitivity to ensure infant survival and survival of the group. Muret-Wagstaff and Moore also noted distinct differences in the meaning of the infant between Hmong and established American groups. For established American groups, the addition of an infant represents a drain on family financial and emotional resources, whereas for Hmong families an infant represents a link with other Hmong clans, insurance for care when the parents became elderly, and contribution of work to the family unit. In addition, several authors have noted that traditionally, Hmong women have had no other role (Jambuthan \& Stewart, 1997; Muret-Wagstaff \& Moore, 1989).

A culture that designates mothering as a highly-valued skill resulting in better infant survival rates would confer status on mothers with high survival rates of children. If meaning for women arises from their role as a mother, then it follows that close attention to the infant who confers that meaning would assume paramount importance. Maternal sensitivity would be part of a very meaningful activity. The presence of an infant could thus contribute to a mother's resilience after massive family losses. A combination of resilience and the meaning of the infant as a continuation of the family may partially account for the ability of mothers in this sample to demonstrate such exquisite sensitivity to their infants. The combined influences of a sense of coherence, meaning of the maternal role, and resilience on matemal sensitivity need to be examined.

Other influences on such positive maternal-infant interactions could be attributed to 
the Confucian world-view of both Vietnamese and Hmong cultures. Given the heritage of filial piety found in both Vietnamese and Hmong cultures, the birth of a son would confer status on the mother. Not only would the infant represent insurance for ancestor worship in the future, a virtuous mother would also be highly attuned to the infant's needs as part of her fulfillment of moral conduct. In Confucian philosophy, a major component of the duty of the wife to her husband is to bear children (especially sons) and care for them so they will grow up to honor the father, mother, brothers and ancestors as expected by filial children (Ebrey, 1991; Kellerher, 1987). Fulfullment of one's duty as a wife would include giving special attention to the infant to ensure survival of the new vulnerable member of the husband's family. Maternal sensitivity would thus become a function of the duty of a filial wife.

Family support has long been cited as an important component in developing high levels of maternal sensitivity (Belsky, 1984; Goldstein, Diener, \& Mangelsdorf, 1996). Within the context of filial piety, family support would be expected from the mother-inlaw. In traditional Chinese-based societies, the husband's mother was responsible for the welfare of the total household. Part of that responsibility included coaching the son's wife on how to care for her infant (Yao, 1983). Mothers from both groups reported continuation of this same pattern. Frequent references were made to help and guidance from both the mother and the mother-in-law with child care, home remedies for illnesses, parameters for expected age-related behaviors, and discipline etc. Many mothers noted that this advice was often contrary to Western ways of infant and child care, which they preferred, and that occasional conflicts occurred between them and their mothers or mothers-in-law around issues of infant and child care. However, mothers-in-law were reported to be supportive of the maternal-infant bond.

Data showing a correlation between lower anxiety and depression rates for both Vietnamese and Hmong participants with more of the husband's family members in the United States lends credence to the notion that family support could be an important mediator between levels of anxiety and depression, and maternal sensitivity, and thus a buffer in the parenting system (Belsky, 1984). Since Belsky's work formed the basis for part of the theoretical model, further investigation in this area is warranted. Interestingly, in 
the present study, mothers were less sensitive to their infants when more of the husband's family lived in the United States. This finding is further addressed in the section "Other Findings".

A few participants reported some of the nonsupportive family relations that Aroian, Spitzer and Bell (1996) described in their sample of former Soviet immigrants. Some Hmong mothers reported difficulty managing the increased responsibility in the United States for raising their children without the large societal network and accepted values that were found in Laos. Considering that the median number of children in the Hmong group was 5.5, assistance would be needed for child care and general household management. Husbands of mothers who expressed such concerns were reported to not help. Observations during interviews lent credence to that statement. Many Hmong fathers generally socialized with the investigator, then left, leaving the mothers 'with all the children. Older siblings assisted the mother by entertaining the younger children. If or how this family interaction pattern will change as families become more accuiturated is still unknown.

\section{Research Question Three}

\section{To what extent does acculturation decrease maternal sensitivity in}

\section{Vietnamese and Hmong mothers?}

Although the correlations only approached significance, the trend was toward less maternal sensitivity when mothers were more acculturated. Studies examining the relationship between maternal sensitivity and acculturation were not located in the body of literature that was searched. The focus of studies that were reported in medical, nursing, and allied health journals was on identification of high-risk populations, high-risk behaviors, ways to improve pregnancy outcomes, diminish high-risk behaviors, and more effectively organize and deliver needed prenatal services to high-risk individuals (Cobas et al. 1996; Scribner \& Dwyer 1989). Zambrana et al. (1997) reported that language abilities were more critically related to infant birth weights, and by inference to the mother's health status, than was ethnicity or ethnic identification.

In Southeast Asian populations, increased acculturation has been associated with 
increased birth weights. Possible explanations for the better health status of Southeast Asian newborns with more acculturated mothers were attributed by $\mathrm{Li}$, et al. (1990) to (a) better health and nutrition of the mother subsequent to arrival in the United States, and (b) employment of the husband resulting in better socioeconomic status. Rumbaut (1996) and Singh and Yu (1996) attributed greater birth weights to a better diet, a lack of smoking and a lack of alcohol or drug use by the mother during her prenatal period. Although birth weights, intant health, and maternal sensitivity are related, they are not identical. Increased birth weights generally reflect environmental influences on the mother. Before immigration, Southeast Asians were subjected to scarce food and medical resources. commodities that have been proven to cause low birth weight in infants (Li et al., 1990; Muecke, 1992). Before immigration from war-torn environments, mothers had no control over access to those resources. Once in the United States, available food supplies and medical care combined to increase the average birth weight ( $\mathrm{Li}$, et al.. 1990).

Self-identification as well as language ability is measured by the SL-ASIA (Suinn, et al., 1992). Part of that self-identification is measured by a desire to fit in with the surrounding society. It is possible that identifying with the surrounding culture is evidence that life can be comprehended, managed, and is meaningful and could indicate a sense of coherence (Antonovsky, 1987), thus a sense of well-being. It seems logical to hypothesize that a sense of coherence would indicate an internal sense that the transition from the old to the new is manageable, comprehensible, and meaningful. If such were the case, enhanced sensitivity toward one's infant would be expected when acculturation was increased. The fact that an opposite trend was found may reflect the overwhelming strength of the surrounding individualistic society that does not value family connections and filial piety to the same degree found in traditional Asian societies. Further investigation of the relationship of acculturation and maternal sensitivity is warranted. Although maternal sensitivity and acculturation were not associated in the present study, relationships were found between acculturation, anxiety and depression.

\section{Research Question Four}

\section{Among Vietnamese and Hmong mothers, what is the relationship}




\section{between PTS, or its components of depression and anxiety, and}

\section{acculturation?}

As reported in the review of literature, stress from the acculturation process has been titled acculturative stress (Berry \& Williams, 1991). In the present study, mothers who were more acculturated experienced more anxiety and depression. Responses to specific items on the SL-ASIA may offer insight into these associations.

When answering a question about perceived ethnic identity, a frequent response was "I now live in America, so I am American." Fifteen out of 32 participants reported that they associated about equally with Asian and non-Asian groups. A greater proportion (24 out of 32) stated they wanted to associate about equally with both Asian and non-Asian groups. Some went on to say that they wanted to have friends who were American but did not have the language abilities to communicate at a desired level. Given the considerable difficulty with English (15 out of 32 participants used interpreters for the interview). communication with English-speaking people could be expected to be very stressful.

Donnelly (1994) reported that Hmong women in particular resisted acculturation. Liebkind (1996) attributed increased levels of acculturative stress in adult Vietnamese women in Finland to a negative attitude toward acculturation. Contrary to Donnelly's findings, the women in this study did not verbalize negative attitudes toward acculturation. However, most did report pride in their ethnic identity. Only four Vietnamese mothers and two Hmong mothers did not. The Hmong key informant stated that it was not proper to acknowledge pride, thus it is possible the negative responses were a result of modesty rather than an indication of true feelings.

The mothers in the present study did describe instances of prejudice, rejection, and discrimination by non-Asians. Many reported that they had been discouraged from seeking contacts with Americans by comments such as "You talk funny" or "You are different." Another said that when she went outside in her neighborhood, children taunted her because she spoke differently than they. Others simply described how looks were exchanged between non-Asians that they interpreted as negative responses to themselves. Key informants were not surprised by these reports. They told the investigator that such experiences were the rule rather than the exception in the Asian community. The push-pull 
of wanting to associate with non-Asians but expecting derisive, negative responses could only produce anxiety. Most likely more-acculturated mothers would have had more interactions with the surrounding dominant culture, therefore more negative experiences. The strong association between acculturation and anxiety could be explained in that manner.

That acculturation is a complex process was illustrated by the association between depression and acculturation. Substantially more depression was noted when it was reported that mothers believed they fit in very well with other nationals from their own group. This was especially true for Hmong mothers. The more they felt they fit in with non-Asians, the less depression they reported. A desire to both become part of the broad social structure, yet maintain traditional family relationships, has been documented in other writings about both Vietnamese and Hmong women (Donnelly, 1994: Kibria, 1993). Perhaps depression is the result of a perception that it is not possible to fit in with people from the dominant culture. While contacts with non-Asians are anxiety-producing, the lack of contacts or the awareness of a better fit with traditional cohorts may contribute to depression.

\section{Other Findings}

There were no significant associations between maternal sensitivity and any demographic variables in the Vietnamese group (Table 5). Two demographic variables were significantly associated with maternal sensitivity in the Hmong group (Table 6). The longer Hmong mothers spent in transition from their own country to the United States, the higher their sensitivity level $\underline{\underline{r}}=.57, \mathrm{~g}=.01$ ). They were in transition an average of 5.5 years, far longer than the .31 years Vietnamese mothers spent in transit. For the total group, the more of the husband's family that lived in the United States, the lower the sensitivity level $(r=-.31, p=<.05)$. In all the literature that was reviewed, no one documented either one of these findings.

The most logical explanation for such unexpected finding is related to the recent history of the Hmong people after the Vietnam conflict. Until accepted by a resettlement nation, the Hmong lived in refugee camps on the border of Thailand and Laos. During that 
time, the question of family and clan survivors was most likely resolved (Hamilton-Merritt, 1993). With few or no family members left in the homeland, available energy focused on the future, with its possibilities for creation of a new life in a new country. Consequently, the ending phase of transition would have given way to an uncertain and chaotic phase while in the camps. As the fate of missing family members became certain, energy would increasingly turn to preparations for a new life in a new land. If the birth of an infant represented continuity of the clan and family in a new country, the impetus for maternal sensitivity would have been enhanced.

The finding that lower maternal sensitivity was related to more of the husband's family who lived in the United States was surprising $(-.31 \mathrm{~g}<.05)$. Contribution of the presence of the husband's family to a sense of coherence is consistent with the Confucian philosophy. Although women looked to their own family members for advice and support, it was the husband's family that defined the family structure. In a new environment, the presence of the husband's family would provide continuity of a traditional family structure relative to family roles and responsibilities. A son's wife does have a lower status in a traditional family system. It is her duty to obey and appease the mother-in-law. Reports of unhappiness and stress have been associated with the position of "daughter-in-law" (Yao, 1983). Perhaps acceptance of a lower status with potential stress brought on by the daughter-in-law's duty to maintain harmony in relations with her in-laws is questioned by the more acculturated women. If so, the traditional coach for maternal sensitivity would lose power and influence. This could mean reduced maternal sensitivity. It is interesting, however, that traditional family pattems continue to be desired by Vietnamese and Hmong women (Donnelly, 1994; Kibria, 1993). The cost of acculturation, however, appears to be substantial stress.

Younger mothers were more acculturated $(r=-.53, \mathrm{p}<.01)$, and more acculturated women reported more members of her husband's family living in the United States $(r=.60, \underline{p}<.001)$. As noted earlier, more acculturated mothers experienced considerably more anxiety. The combination of these associations lends credence to the possibility that the extended family among both Vietnamese and Hmong participants expected a more acculturated mothe: to assist the less acculturated members of the 
husband's family as Aroian et al. (1996) reported. It is possible that more acculturated mothers both have lost the traditional coach for maternal sensitivity and have adopted less traditional attitudes toward their infants. An unfortunate consequence may be decreased maternal sensitivity to an infant.

The number of pregnancies and depression were highly correlated in both groups $(\mathbf{r}$ $=.73, \underline{\mathrm{p}}<.0$ I for Vietnamese; $\underline{\mathrm{r}}=.65, \mathrm{p}<.01$ for Hmong), and with anxiety in the Vietnamese group $(r=.72, \mathrm{~g}<.01)$. Both groups reported more pregnancies when fewer of the husband's family lived in the United States $(r=-.53, \underline{p}<.05$ for Vietnamese; $r=-$ $.39, \mathrm{p}<.10$ for Hmong). Comments from some of the mothers contributed insight to these fïndings.

Many Hmong and some Vietnamese mothers verbalized feelings of being overwhelmed by the care of their children and the daily routines of life in the United States. Most stated that they had not wanted so many children, but the husbands had wanted a large family, so they had more children. Some mothers stated they felt ignorant and useless because they could not speak English, or in some instances could not read, thus could not participate in the local economy or help their children with homework. The only Hmong mother who had attended school in Laos told the investigator how lucky she was thai her father valued education for girls and could afford the school. Most all mothers voluntecred that they wanted their daughters not to get married until they had finished at least school high school.

Jambuthan and Stewart (1997) and Stewart and Jambuthan (1996) described similar feelings as were listed on the HSCL-25, in their sample of Hmong women. That group had just had a baby, and they concluded that negative feelings were more related to the surrounding living environment rather than the postpartum state. Four themes of the mothers in Jambuthan and Stewart's sample were identified: the wish for fewer children, a desire to speak English, be employed and be independent. Depression was not related to the number of children, for the focus of their study was on the social support available to postpartum women. No reports documenting a relationship among the actual number of pregnancies and anxiety or depression was located in any literature searches conducted in CINAHL, PSYClit, or ERIC. Again, additional investigation is needed to first validate that 
the number of pregnancies is associated with depression and anxiety, and then, if indicated, to explore possible reasons for that relationship.

\section{Conclusions}

Two major findings came from this study. The first was that Vietnamese and Hmong mothers of infants do experience PTS, anxiety, and depression. They experience it to the same degree as the general population of Vietnamese and Hmong refugees. In other words, anxiety and depression rates for Vienamese and Hmong women were no different than those reported in large community-based studies. The second major finding was that maternal sensitivity remained very high, even when clinical levels of PTS, anxiety, or depression were present. A small nonsignificant trend toward a drop in maternal sensitivity among more acculturated mothers was found, but again, no cietinitive conclusion can be drawn from this data. Anxiety was most strongly correlated with acculturytion in this group of mot'sers. Given the nature of acculturative stress, stich relationships were not surprising.

The data fit the conceptual model (Figure 1): however, gaps were identified. Contextual determinants as shown in the model, were present. The degree of influence from the context varied by ethnicity and premigration experiences, as noted by the different degrees of anxiety and depression in the mother. How the context influenced maternalinfant interactions was not established. The role of the number of pregnancies a mother has was not included in the model. Findings from this study indicated that it has a profound influence on depression and anxiety. In addition, this study identified protective variables that may have buffered maternal sensitivity from negative contextual influences, but what they are is still unclear.

These paradoxical findings raise many questions. Could they be replicated in other studies of Vietnamese and Hmong mothers, or in refugee groups from other parts of the world, such as Guatemala, Ethiopia, Afghanistan, Bosnia, etc.? If maternal sensitivity remains a robust matemal characteristic in the face of political violence and traumatic experiences for some groups of mothers, as well as multiple unwanted pregnancies, what are possible protective factors that may be present in refugee and other immigrant mothers? The meaning the infant holds for the mother and the family may be one major but 
overlooked protective characteristic. Within the context of Confucian philosophy, the presence of a child, particularly a son, may counter negative influences of depression and anxiety.

Many assumptions were validated, but others were not. By choosing participants from a community of faith, an assumption was made that participants would have a similarly accessible source of social support available. While that assumption was supported by verbal comments made during data collection, social support was not measured in this study. Close supportive relationships were reported by members of faith communities and other clan members. The association of supportive relationships to maternal sensitivity was also not addressed in this study. Influences from Confucian philosophy were not measured, but the presence of such influences was inferred by the comments of inany participants about their roles as mother and as wives. Perhaps nurturing an infant provides meaning to life that overcomes the effects of depression on maternal sensitivity.

Methodological assumptions that were validated unclude the use of clergy and cther personal contacts to gain access to this population of mothers, andthe use of a non-random snowballing technique. Using a disclosure format for the informed consent proved to be a wise choice. While some of the younger and more educated mothers verbalized familiarity with a consent form and seemed ready to sign it, others visibly brightened when told they did not have to sign the consent. In addition, the use of an interview format for data collection proved to be the only way mothers who were unable to read could have been included in the sample.

\section{Recommendations}

\section{Future Research}

Based on the findings of this study, further research with larger samples is needed to explore possible protective factors for maternal sensitivity. Replication studies are needed to determine if maternal sensitivity is strong in other ethnic groups who have experienced political violence. Other replication studies are needed in other cultures that encompass a Confucian worldview to identify if maternal sensitivity levels are similar. 
Comparison studies of highly sensitive mothers in many different cultures could identify common patterns. Structured interviews may provide insight into the meaning of the infant for the mother and how the maternal-infant relationship is perceived by highly sensitive mothers. In this way, insight into protective factors for maternal sensitivity can begin to be delineated. There also is a need to validate the trend for more depressed Hmong mothers to be more sensitive to their infants while more depressed Vietnamese mothers tend to be less sensitive to their infants. If this dichotomy is actually present, then comparison research about predictive patterns for sensitive and nonsensitive mothers could provide further insight into protective factors.

Additional research is needed to validate a prelininary trend for more acculturated mothers to be less sensitive to their infants. Is the price of acculturation decreased maternal sensitivity? From other studies there is evidence that a sense of coherence mediates between acculturation and anxiety and depression (Yin et al., 1997). Does a sense of coherence play a role in mediating stress from the overlapping transitions of migration and parenthood? If so, is maternal sensitivity the price paid for that sense of coherence? Research is needed to explore the strong and powerful association between the number of pregnancies and depression and anxiety. Do other immigrant groups report the same association? How much of the high depression and anxiety found in refugee mothers is due to unwanted pregnancies instead of past and current traumas? In spite of such a strong correlation, why does matemal sensitivity remain so high?

While random sampling is the ideal method for identifying a sample when conducting research, it is not feasible when the focus of research is a vulnerable population and the topic is personal, as is the topic of maternal-infant care patterns. Vulnerable populations are usually hard to access. Trust must be established before contact can be made. This is a time-consuming process, and requires flexibility that often is counter to recommended research protocol. Recruiting the help of trusted community leaders, and other community contacts propved to be a successful method for recruiting participants. Additional time and financial resources would have allowed additional participants. Also, added resources should make the preferred translation method of translation, backtranslation (Brislin, 1973) possible. The use of the interview method for data collection 
was successful and can be recommended for future studies with vulnerable groups, especially if literacy is a potential issue.

\section{Nursing Practice}

Possibly the primary recommendation for practice illuminated by this study is the need to recognize influences from an immigration history on a mother's mental health. When exposure to politically-based violence has occurred, PTS, anxiety, or depression are frequently present. Findings from this study indicated that they are present in mothers of infants to the same degree as they are in the general population of refugees from the same ethnic group. With $43 \%$ of the combined group, and $78.6 \%$ of the Hmong group reporting clinical levels of depression or anxiety, the need for intervention is acute. Such high percentages are well above the 35\% goal cited by Healthy People 2000 (USPHS, 1992)!

It is important to recognize that not all ethnic groups from the same region experience similar levels of distress. as the differences between Vietnamese and Hmong have illustrated. While assessment of matemal anxiety or depression is not a usual part of a prenatal, postpartum, or well-child visit in either a clinic or a home setting, it needs to be included in a routine assessment of mothers who have escaped socially and politically violent regions of the world. By extension, all immigrant mothers who are coping with acculturative stress, or mothers living in areas of high urban violence, could profit from the same assessment of PTS, anxiety, and depression, with attendant support.

Contrary to findings among European-Americans, it is important to recognize that depression or anxiety in mothers who have experienced traumatic premigration or postmigration events does not automatically place infants at high risk due to diminished matemal sensitivity. The use of a structured tool to routinely evaluate interactive behaviors is essential to a sound nursing practice.

While maternal sensitivity has been identified as a construct common to every culture that has been studied, the pattern of its development may or may not be the same. New mothers, in particular, frequently have to learn how to respond sensitively to infants. In cultures that share a Confucian philosophy or worldview, it is often the mother-in-law who coaches new mothers in infant care practices and appropriate responses to infant signals. As demonstrated in this study, such coaching has been extraordinarily successful 
in the Vietnamese and Hmong cultures. Instead of assuming that professional expertise, based on European-American parenting patterns, is the best method for facilitating maternal sensitivity, encouragement of coaching by a trusted family member may facilitate superior maternal sensitivity.

Summary

In conclusion, This study has identified an area of health that needs to be explored in greater depth. For research to continue, adequate funding needs to be available. In an era of quick fixes and dramatic cures, allocating resources for prevention is not popular. In addition, effective support systems for immigrant mothers who have been identified as high-risk for depression, anxiety, or PTS are scarce and need to be developed.

Health care systems that provide services for pregnant mothers and well infants and children need to incorporate culturally-competent screening and intervention services for these mothers. Such services are in keeping with the goals of Healthy People 2000 (USPHS, 1992). Screening ror psychic distress could be easily and efficiently included in a well-child exam. When depression or anxiety is identified. subsequent services could be arranged. Provision of services as described will incur costs and require political will to establish and maintain. For example, the practice of hiring protessional interpreters has generally been abandoned by providers in the San Diego, CA region because they are not cost-effective. For mothers who do not speak English and who are psychologically distressed, an inability to communicate with the medical provider not only prolongs the stress but also increases the risk of self-injury, as was identified by the number who reported thoughts of ending their life. Health care decisions based solely on business principles are not only short-sighted, leading to more extensive and expensive problems in the future, but are also unethical.

Maternal-infant interactions and maternal-infnat health are complex. Neither interventions for young infants and their families who are at risk nor prevention of potential health problems can be reduced to single solutions that uniformily fits all cultural groups. When considering mother-infant interactions, the outcome is broader than a single diagnosis or a single preventive action. It has to do with the growth of healthy children and future healthy adults. Immigrant populations are growing. They hold tremdous potential for 
the future of the nation. Health care providers and health care planners must consider both their unique contributions and needs when planning for health care. Findings from this research can be used to enhance the lives of present and future children and their families. 


\section{References}

Abe, J., Zane, N., \& Chun, K. (1994). Differential responses to trauma: Migration-related discriminants of Post-Traumatic Stress Disorder among Southeast Asian refugees. Journal of Community Psychology. 22, 121-135.

Abueg, F. R., \& Chun, K. M. (1996). Traumatization Stress among Asians and Asian Americans. In A.J. Marsella, M. J. Friedman, E. T. Gerrity, \& R. M. Scurfield (Eds.). Ethnocultural Aspects of Postraumatic Stress Disorder: Issues, Research, and Clinical Applications, (pp. 285-299). Washington, DC: American Psychological Association.

Aderibigbe, Y. A., Gureje, O., \& Omigbodun, O. (1993). Postnatal emotional disorders in Nigerian women: A study of antecedents and associations. British Journal of Psychiatry, 163,645-650.

Adler, S. R. (1995). Refugee stress and folk belief: Hmong sudden deaths. $\underline{\text { Social }}$ Science and Medicine, 40(12), 1623-1629.

Ainsworth, M. D. S. (1976). Systems for coding infant attachment and reciprocal maternal behaviors. Princeton, NJ : Educational Testing Services, ETS Test Collection.

Ainsworth, M. D. S. (1973). The development of infant-mother attachment. In B. M. Caldwell, \& H. N. Ricciuti, (Eds.). Review of Child Development Research: Volume Three: Child Development and Social Policy (pp. 1-94). Chicago, IL: The University of Chicago Press.

Ainsworth, M. D. S. (1967). Infancy in Uganda. Baltimore: The Johns Hopkins Press.

Ainsworth, M. D. S., Bell, S. M., \& Stayton, D. J. (1974). Infant-mother attachment and social development: Socialization a a product of reciprocal responsiveness 
to signals. In M. P. M. Richards (Ed.). The Integration of a Child into a Social World. (pp. 99-135). London: Cambridge University Press.

Ainsworth, M. D. S., Bell, S. M. V., \& Stayton, D. J. (1971). Individual differences in Strange-Situation behavior of one-year-olds. In H. R. Schaffer (Ed.). The Origins of Human Social Relations. (p. 17-57). New York: Academic Press.

Ainsworth. M.D. S., Blehar, M. C., Waters, E., \& Wall, S. (1978). Patterns of attachment: A psychological study of the Strange Situation. Hillsdale, New Jersey: Lawrence Erlbaum Associates, Publishers.

Antonovsky, A. (1987). Unraveling the mystery of health: How people manage stress and stay well. San Francisco: Jossey-Bass Inc.

Ardayfio-Schandorf, E. (1993). Women's health status in Africa - Environmental perspectives from rural communities. Health Care for Women International, 14, 375-386.

Aroian, K. J. (1990). A model of psychological adaptation to migration and resettlement. Nursing Research, 39(1), 5-9.

Aroian, K. J.. Spitzer, A., \& Bell, M. (1996). Family stress and support among former Soviet immigrants. Western Joumal of Nursing Research. 18(6), 655-674.

Baptiste, D. A. (1993). Immigrant fámilies, adolescents and acculturation: Insights for therapists. Marriage and Family Review, 19(3/4), 341-363.

Barney, G. L. (1979). The Hmong of Northern Laos. In National Indochinese Clearinghouse: Center for Applied Linguistics. Indochinese Refugee Education Guides: The Hmong: Their History and Culture (pp. 18-44). Arlington, VA: National Indochinese Clearing House. (Distributed by: Lutheran Immigration and Refugee Service, New York: Lutheran Council in the U.S.A.)

Beck, C. T. (1996). Postpartum depressed mothers' experiences interacting with their children. Nursing Research, 45(2),98-104.

Beck, C. T. (1996). A meta-analysis of the relationship between postpartum depression and infant temperament. Nursing Research, 45(4), 225-230.

Becktell, P. J. (1994). Endemic stress: Environmental determinants of women's health in India. Health Care for Women International, 15, 111-122.

Beiser, M. (1990). Mental health of refugees in resettlement countries. In W. H. 
Holtzman \& T. H. Bornemann (Eds.). Mental Health of Immigrants and Refugees: Proceedings of a Conference Sponsored by Hogg Foundation for Mental Health and World Federation for Mental Health (pp. 51-65). Austin, TX: Hogg Foundation for Mental Health, The University of Texas.

Beiser, M. (1988). Influences of time, ethnicity, and attachment on depression in Southeast Asian refugees. American Journal of Psychiatry, 145(1), 46-51.

Beiser, M. R., Turner J., \& Ganesan, S. (1989). Catastrophic stress and factors affecting its consequences among Southeast Asian refugees. Social Science and Medicine. $\underline{28}(3), 183-195$.

Belsky, J. (1984). The determinants of parenting: A process model.Child Development, 55, 83-96.

Belsky, J. \& Isabella, R. (1988). Maternal, infant, and social-contextual determinants of attachment security. In J. Belsky \& T. Nezworski (Eds.), Clinical Implications of Attachment, (p. 41-94). Hillsdale, New Jersey: Lawrence Erlbaum Associates.

Berry, J. W. (1990). Psychology of acculturatiun: Understanding individuals moving between cultures. In R. Brislin (Ed.). Applied Cross-cultural Psychology $(\mathrm{p}$. 232-253). Newbury Park. NJ: Sage Publications.

Biringen, Z., Robinson, J. L., \& Emde, R. N. (1994). Maternal sensitivity in the second year: Gender-based relations in the dyadic balance of control. American Joumal of Orthopsychiatry, 64(1), 78-90.

Board on Children and Families, Commission on Behavioral and Social Sciences and Education, National Research Council, \& Institute of Medicine. (1995). Immigrant children and their families: Issues for research and policy. Critical Issues for Children and Youths. 5(2), 72-89.

Bowlby, J. (1952). Maternal care and mental health: A report prepared on behalf of the World Health Organization. New York, NY: Schocken Books.

Bowlby, J. (1965). Child care and the growth of love. (2nd ed.). Baltimore, MD: Penguin Books. 
Bowlby, J. (1982). Attachment and loss. (2nd. Ed.). New York: Basic Books, Inc.

Bretzke, J. T. (1995). The Tao of Confucian virtue ethics. International Philosophical Quarterly, XXXV (1, No. 137), 25-41.

Brislin, R. W., Lonner, W. J., \& Thorndike, R. M. (1973). Cross-cultural research methods. New York, NY: John Wiley \& Sons.

Bridges, W. (1980). Transitions. Reading, MA: Addison-Wesley Publishing Company.

Buekens, P., Masuy-Stroobant, G. \& Delvaux, T. (1998). High birth weights among infants of North African immigrants in Belgium. American Journal of Public Health. 88(5). 808-811.

Buhler-Wilkerson, K. (1993). Public Health then and now: Bringing care to the people: Lillian Wald's legacy to Public Health Nursing. American Journal of Public Health. 83(12). 1778-1786.

Bun, C. K., \& Kiong, T. C. (1993). Rethinking assimilation and ethnicity: The Chinese in Thailand. International Migration Review, 27(1), 140-168.

Burns, N., \& Grove, S. K. (1993). The practice of nursing research (2nd. ed.). Philadelphia: W. B. Saunders Company.

Calderon, E. A. (1986). The impact of Indochinese resettlement on the Phillips and Elliot Park neighborhoods in south Minneapolis. In B. T. Downing and D. P. Olney (Eds.), The Hmong in the West: Observations and Reports. Papers of the 1981 Hmong Research Conference. University of Minnesota (pp. 367-386). Minneapolis, MN: Center for Urban and Regional Affairs, University of Minnesota.

California Department of Health Services. (1998). Live births by race of mother. Sacramento, CA: Department of Health Services.

Campbell, S. B., Cohn, J. F., Flanagan, C., Popper, S., \& Meyers, T. (1992). Course and correlates of postpartum depression during the transition to parenthood. Development and Psychopathology 4, 29-47.

Capps, L. L. (1994). Change and continuity in the medical culture of the Hmong in Kansas City. Medical Anthropology Ouarterly, 8(2), 161-177. 
Carlin, J. E. (1990). Refugee and immigrant populations at special risk: Women, children and the elderly. In W. H. Holtzman \& T. H. Bornemann (Eds.). Mental Health of Immigrants and Refugees: Proceedings of a Conference Sponsored by Hogg Foundation for Mental Health and World Federation for Mental Health (pp.224-233). Austin, TX: Hogg Foundation for Mental Health. The University of Texas.

Carlson, A. W. (1994). America's new immigration: Characteristics, destinations and impact, 1970-1989. The Social Science Journal, 31(3), 213-236.

Celano, M. P. \& Tyler, F. B. (1991). Behavioral acculturation among Vietnamese refugees in the United States. The Journal of Social Psychology. 13(3), 373-385.

Chapuis, O. A. (1995). A history of Vietnam from Hong Bang to Tu Duc. Westport, CT: Greenwood Press.

Cheon-Klessig. Y., Camilleri, D. D., McElmurry, B. J. \& Ohlson, V. M. (1988). Folk medicine in the health practice of Hmong refugees W'estern Journal of Nursing Research, 10(5), 647-660.

Chick, N., \& Meleis, A. I. (1986). Transitions: A nursing concem. In P. L. Chinn (Ed.), Nursing research methodology: Issues and implementation (pp. 237-257). Rockville, MD: Aspen Publishers. Inc.

Chung, R. C. \& Bemak, J. (1996). The effects of welfare status on psychological distress among Southeast Asian refugees. The Joumal of Nervous and Mental Disease. $\underline{184}(6), 346-353$.

Chung, R., \& Kagawa-Singer, M. (1993). Predictors of psychological distress among Southeast Asian refugees. Social Science and Medicine, 36(5), 631-639.

Cobas, J. A., Balcazar, H., Benin, M. B., Keith, V. M., \& Chong, Y. (1996). Acculturation and low-birthweight infants among Latino women: A reanalysis of HHANES data with structural equation models. American Journal of Public Health, 86 (3), 394-396.

Coffman, S., Levitt, M. J., \& Guacci-Franco. (1995). Infant-mother attachment: Relationships to matemal responsiveness and infant temperament.Journal of Pediatric Nursing, $10(1), 9-18$.

Cole, E., Espin, O. M., Rothblum, E. D. (Eds.). (1992). Refugee women and 
their mental health: Shattered societies, shattered lives. New York: The Haworth Press, Inc.

Cuellar, I., Harris, L. C., \& Jasso, R. (1980). An acculturation scale for Mexican American normal and clinical populations. Hispanic Journal of Behavioral Sciences, 2. 199-217.

De Girolamo, G. \& McFarlane, A. C. (1996). The epidemiology of PTSD: A comprehensive review of the international literature. In A. J. Marsella, M. J. Friedman, E. T. Gerrity, \& R. M. Scurfield (Eds.), Ethnocultural Aspects of Posttraumatic Stress Disorder: Issues, Research, and Clinical Applications (pp. 33-85). Washington, DC: American Psychological Association.

Derogatis, L. R., Lipman, R. S., Rickels, K., Uhlenhuth, E. H., \& Covi, L. (1974). the Hopkins symptom checklist (HSCL): A self-report symptom inventory. Behavioral Science, 19, 1-15.

De Vita, C. J. (1996). The United States at mid-decade. Pcpulation Bulletin, 50(4). $3-46$.

De Wolff, M. S. \& van Uzendoorn, M. H. (1997). Sensitivity and attachment: A meta-analysis on parental antecedents of infant attachment. Child Development.68(4). 571591.

Donnelly, N. D. (1994). Changing lives of refugee Hmong women. Seattle, WA: University of Washington Press.

Downing, B. (1986). Language issues. In G. L. Hendricks, B. T. Downing, \& A. S. Deinard, (Eds.) The Hmong in Transition. (pp.187-193). New York, NY: Center for Migration Studies of New York, Inc.

Ebrey, P. (1991). The Chinese family and the spread of Confucian values. In G. Rozman (Ed.). The East Asian Region: Confucian Heritage and its Modern Adaptation (pp, 45-83). Princeton, NJ: Princeton University Press.

Everly, G. S. (1995). Psychotraumatology. In G. S. Everly, Jr. \& J. M. Lating (Eds.), Psychotraumatology: Key Papers and Core Concepts in Post-Traumatic Stress (pp. 1-8). New York: Plenum Press. 
Everly, G. S. \& Lating, J. M. (Eds.). (1995). Psychotraumatology: Key papers and core concepts in Post-Traumatic Stress. New York: Plenum Press.

Faugier, J. \& Sargeant, M. (1997). Sampling hard to reach populations. $\underline{\text { Journal of }}$ Advanced Nursing, $26,790-797$.

Fedele, N. M., Golding, E. R., Grossman, F. K., \& Pollack, W. S. (1988). Psychological issues in adjustment to first parenthood. In G. Y. Michaels \& W. A. Goldberg (Eds.), The transition to parenthood (pp. 85-113). Cambridge: Cambridge University Press.

Ferketich, S., Phillips, L., \& Verran, J. (1993). Focus on psychometrics: Development and administration of a survey instrument for cross-cultural research. Research in Nursing \& Health. 16, 227-230.

Ficenec, S. (1996). Advance report: California vital statistics, 1995. State of California: Department of Health Services.

Field, T. (1995). Infants of depressed mothers. Infant Behavior and Development. 18, 1-13.

Field, T., Morrow, C., \& Adlestein, D. (1993). Depressed mothers' perceptions of infant behavior. Infant Behavior and Development. 16, 99-108.

Finck. J. (1982). Clan leadership in the Hmong community of Providence, Rhode Island. In B. T. Downing \& D. P Olney (Eds.), The Hmong in the West: Observations and Reports. Papers of the 1981 Hmong Research Conference (pp.21-28). Minneapolis, MN: Center for Urban and Regional Affairs. (Pub. No. CURA 82-1)

Foss, G. F. (1996). A conceptual model for studying parenting in immigrant populations. Advances in Nursing Science, 19(2), 74-87.

Foss, G.F. (1995). Public Health Nursing practice: Professional noticing. interpreting and intervening. Unpublished manuscript, University of San Diego at San Diego, CA.

Fox, P. (1991). Stress related to family change among Vietnamese refugees. Joumal of Community health Nursing, $\underline{8}(1), 45-56$.

Friedman, A. R. (1992). Rape and domestic violence: The experience of refugee women. In E. Cole, O. M. Espin, \& E. D. Rothblum, (Eds.), Refugee women and their 
mental health: Shattered societies, shattered lives, (pp. 65-77). New York: The Haworth Press, Inc.

Frye, B. A., \& D'Avanzo, C. D. (1994). Cultural themes in family stress and violence among Cambodian refugee women in the inner city. Advances in Nursing Science, 16(3). 64-77.

Gold, S. J. (1992). Refugee communities: A comparative field study. Newbury Park, CA: Sage publications.

Goldberg, W. A. \& Michaels, G. Y. (1988). Conclusion: The transition to parenthood: Synthesis and future directions. In G. Y. Michaels \& W. A. Goldberg (Eds.),The transition to parenthood (pp. 342-360). Cambridge: Cambridge University Press.

Goldsmith, H. H., \& Alansky, J. A. (1987) Maternal and infant temperamental predictors of attachment: A meta-analytic review. Journal of Consulting and Clinical Psychology, 55(6), 805-816.

Gcldstein, B. L. (1986). Resolving sexual assault, Hmong and the American legal system. In G. L. Hendricks, B. T. Downing. \& A. S. Deinard (Eds.), The Hmong in Transition (pp. 135-143). New York, NY: Center for Migration Studies of New York, Inc.

Goldstein. L. H., Diener, M. L., \& Mangelsdorf, S. C. (1996). Maternal characteristics and social support across the transition to motherhood: Associations with maternal behavior. Journal of Family Psychology 10(1), 60-71.

Gonsalves, S. J. (1992). Psychological Stages of the refugee process: A model for therapeutic interventions. Professional Psychology: Research and Practice, 23(5), 382-389.

Gottlieb, B. H. \& Pancer S. M. (1988). Social networks and the transition to parenthood. In G. Y. Michaels \& W. A. Goldberg (Eds.), The Transition to Parenthood. (pp. 235-269). Cambridge: Cambridge University Press.

Gross, D. (1996). What is a "Good" parent? MCN 21(July/Aug), 178-182.

Guendelman, S., Gould, J. B., Hudes, M., \& Eskenazi, B. (1990). Generational differences in perinatal health among the Mexican American population: Findings from HHANES 1982-84. American Journal of Public Health, 80(Supplement), 61-65. 
Haines, D. W. (Ed.). (1989). Refugees as immigrants. Totowa, NJ: Rowman \& Littlefield Publisher, Inc.

Hall, L. A., Kotch, J. B., Browne, D. \& Rayens, M. K. (1996). Self-esteem as a mediator of the effects of stressors and social resources on depressive symptoms in postpartum mothers. Nursing Research. 45(4), 231-238.

Hamilton-Merrith, L. (1993). Tragic Mountains: The Hmong, the Americans and the secret wars for Laos, 1942-1992. Bloomington, ID: Indiana University Press.

Harwood, R. L., Miller, J. G., \& Irizarry, N. L. (1995). Culture and Attachment: Perceptions of the child in context. New York, NY: The Guilford Press.

Harkness, S., \& Super, C. M. (Eds.). (1996). Parents' cultural belief systems: Their origins, expressions, and consequences. New York, NY: The Guilford Press.

Hauff. E., \& Vaglum, P. (1995). Organized violence and the stress of exile: Predictors of mental health in a community cohort of Vietnamese refugees three years after resettlement. British Joumal of Psychiatry, 166, 360-367.

Hautman, M. A., \& Bomar, P. (1995). Interactional model for recruiting ethnically diverse research participants. The Joumal of Multicultural Nursing and Health. 1(4), 3-27.

Heermann, J. A., Jones, L. C. \& Wikoff, R. L. (1994). Measurement of parent behavior during interactions with their infants. Infant Behavior and Development. 17, 311-321.

Heisler, B. S. (1992). The future of immigrant incorporation: Which models? Which concepts? International Migration Review, 26(2), 623-645.

Henderson, S. M. \& Brown, J. S. (1987). Infant feeding practices of Vietnamese immigrants to the Northwest United States. Scholarly Inquiry for Nursing Practice: An International Journal, 1(2), 153-174.

Hendricks, G. L., Downing, B. T., \& Deinard, A.S. (Eds.). (1986). The Hmong in transition. New York, NY: Center for Migration Studies of New York, Inc.

Hesbacher, P. T., Rickels, K., Morris, R. J., Newman, H. \& Rosenfeld, H. (1980). Psychiatric illness in family practice. Journal of Clinical Psychiatry. 41(1), 6-10.

Hinkle, D. E., Wiersma, W., \& Jurs, S. G. (1994). Applied statistics for the behavioral sciences. (Third Ed.). Boston: Houghton Mifflin Company. 
Hinsch, B. (1995). Harmony (he) and gender in early Chinese thought. Journal of Chinese Philosophy, 22(1995), 109-128.

Hinton, W. L., Tiet, Q., Tran, C. G., \& Chesney, M. (1997). Predictors of depression among refugees from Vietnam: A longitudinal study of new arrivals. The Journal of Nervous and Mental Disease. 185(1), 39-45.

Hobtoll, S. E., Ritter, C., Lavin, J., Hulsizer, M. R., \& Cameron, R. P. (1995). depression prevalence and incidence among inner-city pregnant and postpartum women. Journal of Consulting and Clinical Psychology, 63(3), 445-453.

Hock, E., Schirtzinger, M. B., Lutz, W. J., \& Widaman, K. (1995). Maternal depressive symptomatology over the transition to parenthood: Assessing the influence of marital satisfaction and marital sex role traditionalism. Journal of Family Psychology.9(1), 79-88.

Hong, G. K. Bom, S. L. \& Lorenzo, M. K. (1995). Somatizatinn in Chinese Ame:ican clients: Iniplications for psychotherapeutic services. Journal of C'ontemporary Psychotherapy 25(2), 105-118.

Hopkins, S. M. (1997, March 24). Down-home foreign flavor: Local nanufacturers feed growing appetite for ethnic food. The Charlotte Observer. pp. 10D-11D.

Isabella, R. A. (1994). Origins of maternal role satisfaction and its influences upon maternal interactive behavior and infant-mother attachment. Infant Behavior and Development, 17 , 381-387.

Isabella, R. A. (1993). Origins of attachment: Maternal interactive behavior across the first year. Child Development, 64, 605-621.

Jackson, J. F. (1993). Multiple caregiving among African Americans and infant attachment: The need for an emic approach. Human Development, 36 87-102.

Jambunathan, J. (1995). Hmong cultural practice and beliefs: The postpartum period. Clinical Nursing Research, 4(3), 335-345).

Jambunathan, J. \& Stewart, S. (1997). Hmong women: Postpartum family support and life satisfaction. Journal of Family Nursing. 3(2), 149-166. 
Jamieson, N. L. (1993). Understanding Vietnam. Berkeley, CA: University of California Press.

Jones, E. G., \& Kay, M. (1992). Instrumentation in cross-cultural research. Nursing Research 41(3), 186-188.

Juthani, N. V. (1992). Immigrant mental health: Conflicts and concerns of Indian immigrants in the U.S.A. Psychology and Developing Societies. 4(2), 133-148.

Karl, D. (1991). The consequence of maternal depression for early mother-infant interaction: A nursing issue. Journal of Pediatric Nursing. 6(6), 384-390.

Kelleher, T. (1987). Confucianism. In A. Sharma (Ed.). Women in world Religions (pp. 135-159). Albany, NY: State University of New York Press.

Kibria, N. (1993). Family tightrope: The changing lives of Vietnamese Americans. Princeton, NJ: Princeton University Press.

Kibria, N. (1990). Power, patriarchy, and gender conflict in the Vietnamese immigrant community. Gender \& Society 4 (1); 9-24.

Kim, S. \& Rew, L. (1994). Ethnic identity, role integration, quality of life, and depression in Korean-American women. Archives of Psychiatric Nursing 8(6), 348-356.

Kinsley, D. (1996). Health healing and religion: A cross-cultural perspective. Upper Saddle River, NJ: Prentice-Hall Inc.

Koniak-Griffin, D. (1993). Maternal role attainment. Image. 25(3), 257-262.

Kroll, J., Habenicht, M., Mackenzie, T., Yang, M., Chan, S., Vang, T., Nguyen, T., Ly, M., Phommasouvanh, B., Nguyen, H., Vang,Y., Souvannasoth, L. \& Cabugao, R. (1989). Depression and post traumatic stress disorder in Southeast Asian refugees. American Journal of Psychiatry, 146(12), 1592-1597.

Lantican, L. S. M., \& Corona, D. F. (1992). Comparison of the social support networks of Filipino and Mexican-American primigravidas. Health Care for Women International, 13, 329-338.

Leininger, M. M. (1991). The theory of culture care diversity and universality. In M. M. Leininger, (Ed.). Culture Care Diversity and Universality: A Theory of Nursing (pp. 5-68). New York: National League for Nursing Press, Pub. No. 15-2402.

Lemoine, J. (1986). Shamanism in the context of Hmong resettlement. In In G. L. 
G. L. Hendricks, B. T. Downing, \& A. S. Deinard, (Eds.) The Hmong in Transition. (pp.399-416). New York, NY: Center for Migration Studies of New York, Inc.

Hendricks, B. T. Downing, \& A. S. Deinard, (Eds.) The Hmong in Transition (pp.337-348). New York, NY: Center for Migration Studies of New York, Inc.

Lese, K. P., \& Robbins, S. B. (1994). Relationship between goal attributes and the academic achievement of Southeast Asian adolescent refugees. Joumal of Counseling Psychology. 41(1), 45-52.

Levitt, M. J., Coffman, S., Guacci-Franco, N., \& Loveless, S. (1993). Social support and relationship change after childbirth: An expectancy model. Health Care for Women International, 14, 503-512.

Levy-Shiff, R. (1994). Individual and contextual correlates of marital change across the transition to parenthood. Developmental Psychology, 30(4). 591-601.

Li, D., Ni, H., Schwartz, S. M., \& Daling, J. R. (1990). Secular change in birth weight among Southeast Asian immigrants to the United States. American Journal of Public Health, 80$(6), 685-688$,

Liebkind, K. (1996). Acculturation, identity, and stress: Vietnamese refugees in Finland. Journal of Cross-cultural Psychology, 27(2), 161-180.

Lipson, J. G. (1992). The health and adjustment of Iranian immigrants. IVestern Joumal of Nursing Research, 14(1), 10-29.

Lipson, J. G., \& Miller, S. (1994). Changing roles of Afghan refugee women in the United States. Health Care for Women International, 15, 171-180.

Live Births by Race of Mother. (1998). California Counties, 1996, State of California. Department of Health Services. Sacramento, Department of Health Services.

Logsdon, M. C., McBride, A. B., \& Birkimer, J. C. (1994). Social support and postpartum depression. Research in Nursing \& Health. 17, 449-457.

Luong, H. V. (1984). "Brother" and "Uncle": An analysis of rules, structural contradictions, and meaning in Vietnamese kinship. American Anthropologist, 86, 290-305.

MacPhee, D., Fritz, J., \& Miller-Heyl, J. (1996). Ethnic variations in personal social networks and parenting.Child Development, 67(6), 3278-3295. 
Marsella. A. J., Friedman, M. J., Gerrity, E. T., \& Scurfield, R. M. (1996). Ethnocultural aspects of Posttraumatic Stress Disorder: Issues, research, and clinical applications. Washington, DC: American Psychological Association.

Martin, P. \& Midgley, E. (1994). Immigration to the United States: Journey to an uncertain destination. Population Bulletin, 49(2), 3-46.

Massey, D. S. (1995). The new immigration and ethnicity in the United States. Population and Development Review, 21(3), 631-652.

Matsuoka, J. (1993). Demographic characteristics as determinants in qualitative differences in the adjustment of Vietnamese refugees. Joumal of Social Service Research. $17(3 / 4), 1-21$.

Matsuoka, J. K. (1990). Differential acculturation among Vietnamese refugees. Social Work, 35(4), 341-345.

Matthews, K. A., Scheier, M. F., Brunson, B. I., \& Carducci, B. (1989;. Why do unpredictable events lead to reports of physical symptoms? In T. W. Miller (Ed.), Stressful Life Events, (pp. 91-95). Madison, WI: International Universities Press, Inc.

Mattson, S. (1993). Mental health of Southeast Asian refugee women: An overview. Health Care for Women International, 14, 155-165.

Mavreas, V., Bebbinton, P., \& Der, G. (1989). The structure and validity of acculturation: Analysis of an acculturation scale. Social Psychiatry \& Psychiatric Epidemiology, 24, 233-240.

McKelvey, R. S., Webb, J. A., Strobel, R. M. (1996). The prevalence of psychiatric disorders among Vietnamese Amerasians: A pilot study. American Journal of Orthopsychiatry, 66(3), 409-415.

Mebert, C. J. (1991). Variability in the transition to parenthood experience. In K. Pillemer \& K. McCartney (Eds.), Parent-child relations throughout life (pp. 43-57). Hillsdale, NJ: Lawrence Erlbaum Associates, publishers.

Mei, W. P. (1967). The basis of social, ethical, and spiritual values in Chinese philosophy. In C. A. Moore (Ed.), The Chinese Mind: Essentials of Chinese Philosophy and Culture (pp. 149-166). Honolulu, HI: East-West Center Press. 
Meleis, A. I. (1991). Between two cultures: Identity, roles, and health. Health Care for Women International, 12, 365-377.

Meleis, A. I., Lipson, J. G., \& Paul, S. M. (1992). Ethnicity and health among five Middle Eastern immigrant groups. Nursing Research. 41(2) 2 98-103.

Mercer, R. T. \& Ferketich, S. L. (1990). Predictors of parental attachment during early parenthood. Journal of Advanced Nursing, 15, 268-280.

Mghir, R., Freed, W., Raskin, A., \& Katon, W. (1995). Depression and posttraumatic stress disorder among a community sample of adolescent and young adult Afghan refugees. The Journal of Nervous and Mental Disease, 183, 24-30.

Mollica, R. F., Wyshak, G., Lavelle, J., Truong, T., Tor, S., \& Yang, T. (1990). Assessing symptom change in Southeast Asian refugee survivors of mass violence and torture. American Journal of Psychiatry, 147(1), 83-88.

Mollica, R. F.. Wyshak. G., de Marneffe, D., Khoun, F., \& Lavelle, J. (1987). Indochinese versions of the Hopkins Symptom Checklist-25: A screening instrument for the psychiatric care of refugees. American Joumal of Psychiatry. 144(4), 497-500.

Mollica, R. F., Wyshak, G., \& Lavelle, J. (1987). The psychosocial impact of war trauma and torture on Southeast Asian refugees. American Journal of Psychiatry, 144(12), 1567- 1572.

Mouanoutoua, V. L. \& Brown, L. G. (1995). Hopkins Symptom Checklist-25, Hmong Version: A screening instrument for psychological distress. Journal of Personality Assessment. 64(2), 376-383.

Muecke, M. A. (1992). New paradigms for refugee health problems. Social Science and Medicine, 35(4), 515-523.

Muret-Wagstaff, S. \& Moore, S. G. (1989). The Hmong in America: Infant behavior and rearing practices. In J. K. Nugent, B. M. Lester, \& T. B. Brazelton (Eds.), The Cultural Context of Infancy, Volume 1: Biology, Culture, and infant Development (pp. 319-339). Norwood, NJ: Ablex Publishing Corporation.

Murray, L. (1992). The impact of postnatal depression on infant development. Joumal of Child Psychology and Psychiatry, 33(3), 543-561.

Nicassio, P. M. (1985). The psychosocial adjustment of the Southeast Asian 
refugee: An overview of empirical findings and theoretical models. Joumal of CrossCultural Psychology, 16(2), 153-173.

Nicholson, B. L. (1997). The influence of pre-emigration and post emigration stressors on mental health: A study of Southeast Asian refugees. Social Work Research $\underline{21}(1), 19-31$.

Norton, I. M. \& Manson, S. M. (1992). An assiciation between domestic violence and depression among Southeast Asian refugee women. The Journal of Nervous and Mental Disease, $180(11), 729-730$.

Norusis, M. J./SPSS Inc. (1994). SPSS 6.1 base system user's guide: Part 2. Chicago, IL: SPSS Inc.

Oberg, C. N., Muret-Wagstaff, S., Moore, S. G., \& Cumming, B. (1986). A cross-cultural assessment of maternal-child interaction: Links to health and development. In In G. L. Hendricks, B. T. Downing, \& A. S. Deinard, (Eds.) The Hmong in Transition, (pp.399-416). New York, NY: Center for Migration Studies of New York. Inc.

Overmeyer, D. L. (1986). Religions of China: The world as a living system. San Francisco: Harper \& Row Publishers.

Overmeyer, D. L.. Arbuckle. G., Gladney, D. C., McRae, J. R., Taylor, R. L., Teiser, S. F., \& Verellen, F. (1995). Chinese religious - the state of the field. Part II: Living religious traditions: Taoism, Confucianism, Buddhism, Islam, and Popular religions. The Journal of Asian Studies, 54(2), 314-395.

Patel, N., Power, T. G., \& Bhavngari, N. P. (1996). Socialization values and practices of Indian immigrant parents: Correlates of modernity and acculturation. Child Development, 67(2), 302-313.

Penner, L. A., \& Anh, T. (1977). A comparison of American and Vietnamese value systems. The Journal of Social Psychology. 101, 187-204.

Pettit, G. S., Bates, J. E., \& Dodge, K. A. (1997). Supportive parenting, ecological context, and children's adjustment: a seven-year longitudinal study. Child Development, 68(5), 908-923.

Phan, T. T. (1996). Ethnic and cultural specificities of domestic violence: a research and clinical discourse with reference to the Vietnamese emigrant community. The 
International Journal of Psychiatric Nursing Research, 2(2), 187-197.

Pianta, R. C. \& Egeland, B. (1994). Relation between depressive symptoms and stressful life events in a sample of disadvantaged mothers. Journal of Consulting and Clinical Psychology. 62(6), 1229-1234.

Pickens, J. \& Field, T. (1993). Facial expressivity in infants of depressed mothers. Developmental Psychology, 29(6), 986-988.

Posada, G., Gao, Y., Wu, F., Posada, R., Tuscan, M., \& Schoelmerich, A. (1995). The secure-base phenomenon across cultures: Children's behavior, mothers' preferences, and experts' concepts. Monographs of the Society for Research in Child Development, 60(2-3, Serial No 244), 25-48.

Quincy, K. (1995). Hmong: History of a people. Cheney, WA: Eastern University Press.

Radke-Yarrow, M. (1991). Attachment patterns in children of depressed mothers. In C. M. Parkes, J. Stevenson-Hinde, \& P. Marris, (Eds.). Attachment Across the Life Cycle (pp. 115-126. New York: Routledge Publishing Cumpany.

Reed, B. E. (1987). Taoism. In A. Sharma (Ed.). Women in World Religions (pp. 161-181). Albany, NY: State University of New York.

Rogler, L. H. (1994). International migrations: A framework for directing research. American Psychologist. 49(8), 701-708.

Rogler, L. H., Cortes, D. E., \& Malgady, R. G. (1991). Acculturation and mental health status among Hispanics: Convergence and new directions for research. American Psychologist 46(6), 585-597.

Ronan, C. A. (1978). The shorter science and civilization in China: An abridgement of Joseph Needham's original text. Volume I: Volumes I and II of the major series. Cambridge: Cambridge University Press.

Root, M. P. (1996). Women of color and traumatic stress in "Domestic Captivity": Gender and race as disempowering statuses. In A. J. Marsella, M. J. Friedman, E. T. Gerrity, \& R. M. Scurfield (Eds.),Ethnocultural Aspects of Postraumatic Stress Disorder: Issues, Research, and Clinical Applications (pp. 363-387). Washington, DC: American Psychological Association. 
Rossiter, J. C., Ledwidge, H., \& Coulon, L. (1993). IndoChinese women's breast feeding practices following immigration to Sydney: A pilot study. Journal of Advanced Nursing, 19(3), 3-9.

Rousseau, C., Drapeau, A., \& Corin, E. (1997). The influence of culture and context on the pre- and post-migration experience of school-aged refugees from Central America and Southeast Asia in Canada. Social Science and Medicine. 44(8), 1115-11 27.

Rubin, R. (1984). Matemal identity and the maternal experience. New York: Springer Publishing Company.

Rumbaut, R. G. (1996). A legacy of war: Refugees from Vietnam, Laos, and Cambodia. In S. Pedraza \& R. G. Rumbaut (Eds.). Origins and Destinies: Immigration, Race, and Ethnicity in America. Belmont, CA: Wadsworth Publishing Co.

Rumbaut, R. G. (1994). The crucible within: Ethnic identity, self-esteem. and seginented assimilation among children of immigrants. International Migration Review. 28(4), 748-794.

Rumbaut, K. G. (1989). Portraits, patterns and predictors of the refugee adaptation process: Results and reflections from the IHARP panel study. In D. Haines, (Ed.). Refugees as Immigrants (pp. 138-182). Totowa, N.J.: Rowman and Littlefield Publishers Inc.

Rutledge, P. (1985). The role of religion in ethnic self-identity: A Vietnamese community. Lanham, MD: University Press of America, Inc.

SANDAGG/Sourcepoint. (Jan./Feb. 1997). Population and income characteristics of the San Diego region. INFO. San Diego, CA: Sourcepoint.

Schumacher, K. L., \& Meleis, A. I. (1994). Transition: A central concept in nursing. Image, Journal of Nursing Scholarship. 26(2), 119-127.

Scott, G. M. (1986). A new year in a new land: Religious change among the Lao Hmong refugees in San Diego. In B. T. Downing and D. P. Olney (Eds.), The Hmong in the West: Observations and Reports, Papers of the 1981 Hmong Research Conference. University of Minnesota (pp. 63-81). Minneapolis, MN: Center for Urban and Regional Affairs, University of Minnesota. 
Scribner, R. \& Dwyer, J. H. (1989). Acculturation and low birth weight among Latinos in the Hispanic HANES. American Journal of Public Health. 79(9), 1263-1267.

Seideman, R. Y. Kleine, P. F. (1995). A theory of transformed parenting: Parenting a child with developmental delay/mental retardation. Nursing Research, 44(1), $38-44$.

Seifer, R., Schiller, M., Sameroff, A. J., Resnick, S., \& Riordan, K. (1996). Attachment, maternal sensitivity, and infant temperament during the first year of life. Develupmental Psychology, 32(1), 12-25.

Shaw, D. S., \& Vondra, J. I. (1993). Chronic family adversity and infant attachment security. Journal of Child Psychology and Psychiatry, 34(7), 1295-1215.

Singh, G. K., \& Yu, S. M. (1996). Adverse pregnancy outcomes: Differences between US-and foreign-born women in major US racial and ethnic groups. American Journal of Public Health, 86(6), 837-843.

Solkoff, N. (1992). Children of survivors of the Nazi Holocaust: A critical review of the literature American Journal of Orthopsychiatry, 62(3), 342-358.

Stamp, G. H. (1994). The appropriation of the parental role through communication during the transition to parenthood. Communication Monographs, 61 , June, 1994.

State of Califormia, Department of Finance. (1998). Race/ethnic population estimates: Components of change by race, April, 1990 to July, 1996. Sacramento, CA, January, 1998. Available on-line: http:/www.dof.ca.gov/html/Demograp/race-eth.htm.

Stein, J. \& Urdang, L. (Eds.). (1967). The random house dictionary of the English language. New York, Random House Inc.

Stewart, S. \& Jambunathan, J. (1996). Hmong women and postpartum depression. Health Care for Women International, 17 319-330.

Stoleru, S. \& Morales-Huet, M. (1991). Parental attitudes and mother-infant interaction in mothers from Maghreb living in France. In J. K. Nugent, B. M. Lester \& T. B. Brazelton, (Eds.), The cultural context of infancy: Volume 2: Multicultural and interdisciplinary approaches to parent-infant relations. (pp. 299-320). Norwood, NJ: Ablex Publishing Corporation. 
Strayer, F. F., Verissimo, M., Vaughn, B. E., \& Howes, C. (1995). A quantitative approach to the description and classification of primary social relationships. Monographs of the Society for Research in Child Development, 60(2-3, Serial No 244), 49-70.

Suinn, R. M., Ahuna, C., \& Khoo, G. (1992). The Suinn-Lew Asian Self-identity Acculturation Scale: Concurrent and factorial validation. Educational and Psychological Measurement, 52, 1041-1046.

Suinn, R. M., Rickard-Figueroa, K., Lew, S., \& Vigil, P. (1987). The Suinn-Lew Asian Self-identity Acculturation Scale: An initial report. Educational and Psychological Measurement. 47, 401-407.

Susman, E. J., Trickett, P. K., Iannotti, R. J., Hollenbeck, B. E., \& ZahnWaxler, C. (1985). Child-rearing patterns in depressed, abusive. and normal mothers. American Journal of Orthopsychiatry, 55(2), 237-251.

Tapp, N. (1982). Geomancy as an aspect of upland-lowland relationships. In B. T. Downing \& D. P Olney (Eds.), The Hmong in the West: Observations and Reports. Papers of the 1981 Hmong Research Conference (pp. 87-95). Minneapolis, MN: Center for Urban and Regional Affairs. (Pub. No. CURA 82-1)

Taylor, K. W. (1983). The birth of Vietnam. Berkeley, CA: University of California Press.

Thao, C. (1982). Hmong migration and leadership in Laos and in the United States. In B. T. Downing \& D. P Olney (Eds.), The Hmong in the West: Observations and Reports, Papers of the 1981 Hmong Research Conference (pp. 99-121). Minneapolis, MN: Center for Urban and Regional Affairs. (Pub. No. CURA 82-1)

Thao, X. (1986). Hmong perception of illness and traditional ways of healing. In G. L. Hendricks, B. T. Downing, \& A. S. Deinard (Eds.), The Hmong in Transition (pp.365-377). New York, NY: Center for Migration Studies of New York, Inc.

Tomlinson, P. S. (1996). Marital relationship change in the transition to parenthood: A reexamination as interpreted through transition theory. Joumal of Family Nursing. 2(3), 286-305. 
Tran, T. V. (1993). Psychological traumas and depression in a sample of Vietnamese people in the United States. Health and Social Work. 18(3) 184-194.

Tronick, E. Z., Morelli, G. A., \& Ivey, P. K. (1992). The Efe forager infant and toddler's pattern of social relationships: Multiple and simultaneous. Developmental Psychology. 28(4), 568-577.

U.S. Bureau of the Census. (1994). County and city data book: A statistical abstract supplement. Washington, DC.

U.S. Committee for Refugees. (1996). Refugee Reports. 17(12), 10-11.

United States Public Health Service. (1992). Healthy People 2000. Boston: Jones and Bartlett Publishers.

Valenzuela, M. (1997). Maternal sensitivity in a developing society: The context of urban poverty and infant chronic undernutrition. Developmental Psychology. 33(5), 845855.

valt den Boom, D. C. (1997). Sensitivity and Attachment: Next steps for deveiopmentalists. Child Development. 64(4), 592-594.

van den Boom, D. C., \& Hoeksma, J. B. (1994). The effect of infant irritability on mother-infant interaction: A growth-curve analysis. Developmental Psychology 30 ( 4 , $581-590$.

van IJzendoorn, M. H. van, Goldberg, S., Kroonenberg, P. M., \& Frenkel, O. J. (1992). The relative effects of maternal and child problems on the quality of attachment: A meta-analysis of attachment in clinical samples. Child Development, 63, 840-858.

Vondra, J. I., Shaw, D. S., Kevenides, M. C. (1995). Predicting infant attachment classification from multiple, contemporaneous measures of maternal care. Infant Behavior and Development 18; 415-425.

Wahl, M. (1998). Financial culture clash: Immigrants may find it hard to handle money the American way. The Charlotte Observer, 1D-2D.

Waitzkin, H., \& Magana, H. (1997). The black box in somatization: unexplained physical symptoms, culture, and narratives of trauma. Social Science and Medicine.45(6), 811-825. 
Waltz, C. F., Strickland, O. L., \& Lenz, E.R. (1991). Measurement in nursing research. (2nd Ed.). Philadelphia, PA: F.A. Davis Company.

Waters, T. (1995). Towards a theory of ethnic identity and migration: The formation of ethnic enclaves by migrant Germans in Russia and North America. International Migration Review. 29(2), 515-544.

Weinstein-Shr, G. \& Henkin, N. Z. (1991). Continuity and change: Intergenerational relations in Southeast Asian refugee tamilies. Marriage \& Family Review. (17). $351-367$.

Westermeyer, J., (1995). Cross-cultural care for PTSD: Research, training, and service needs for the future. In G. S. Everly, \& J. M. Lating (Eds.). Psychotraumatology: Key Papers and Core Concepts in Post-Traumatic Stress. (pp. 375-393). New York: Plenum Press.

Westermeyer, J. (1986). Indochinese refugees in community and clinic: A report from Asian and the united States. In C. L. Williams \& J. Westermeyer (Eds.). Retugee Mental Health in Resettlement Countries (pp.113-129). Washington DC: Hemisphere Publishing Corporation.

Westermeyer, J. (1988). DSM-III Psychiatric disorders among Hmong refugees in the United States: A point prevalence study. American Joumal of Psychiatry, 145(2), 197 202.

Westermeyer, J., Neider, J., \& Vang, T. F. (1984). Acculturation and mental health: A study of Hmong refugees at 1.5 and 3.5 years post-migration. Social Science and Medicine, 18(1), 87-93.

Westermeyer, J., Vang, T. F., \& Neider, J. (1983). Refugees who do and do not seek psychiatric care: an analysis of premigratory and postmigratory characteristics. Journal of Nervous and Mental Diseases. 171, 86-91.

Williams, H.A. (1990). Families in refugee camps. Human Organization, 49(2), 100-109.

Williams, C. L. \& Berry, J. W. (1991). Primary prevention of acculturative stress among refugees. American Psychologist. 46(6), 623-641. 
Winokur, A., Winokur, D. F.. Rickels, K., \& Cox, D. S. (1984). Symptoms of emotional distress in a family planning service: Stability over a four week period. British Journal of Psychiatry, 144, 395-399.

Yang, S. K. (1979). The Hmongs of Laos: 1986 - 1978. In National Indochinese Clearinghouse: Center for Applied Linguistics. Indochinese Refugee Education Guides: The Hmong: Their History and Culture (pp. 3-17). Arlington, VA: National Indochinese Clearing House. (Distributed by: Lutheran Imrnigration and Refiugee Service, New York: Lutheran Council in the U.S.A.).

Yao, E. S. L. (1983). Chinese women: Past \& present. Mesquite, TX: Ide House, Inc.

Yee, B. W. K. (1992). Markers of successful aging among Vietnamese refugee women. In E. Cole, O. M. Espin, \& E. D. Rothblum (Eds.), Refugee Women and Their Mental Health: Shattered Societies, Shattered Lives (pp. 221-238). New York: The Haworth Press, Inc.

Yin, Y. \& Akutsu, P. D. (1997). Psychological adjustment of Southeast Asian refugees: The contribution of Sense of Coherence. Jonmal of Community Psychology, 25(2), 125-139.

Yin, Y., Akutsu, P. D., Zhang, X., \& Huang, L N. (1997). Psychological dysfunction in Southeast Asian refugees as mediated by Sense of Coherence. American Journal of Community Psychology, 25(6), 839-859.

Yu-Wei, H. (1967). Filial piety and Chinese society. In C. A. Moore (Ed.), The Chinese Mind: Essentials of Chinese Philosophy and Culture (pp. 167-187). Honolulu, HI: East-West Center Press.

Zabielski, M.T. (1984). Recognition of maternal identity in pre-term and fullterm mothers. Maternal-Child Nursing Journal, 22(1), 2-36.

Zhang, D. (1995). Depression and culture-A Chinese perspective. Canadian Journal of Counseling. 29(3), 227-233.

Zimmerman, L. \& McDonald, L. (1995) Emotional availability in infants; relationships with multiple care givers. American Joumal of Orthopsychiatry, 65(1), 147-152. 
Zuckerman, B. Stevenson, J. \& Bailey, V. (1987). Sleep problems in early childhood: Continuities, predictive factors, and behavioral correlates. Paediatrics. 80, 664671.

Zumbrana, R. E., Scrimshaw, S. C. M., Collins, N., \& Dunkel-Schetter, C. (1997). Prenatal health behavior and psychosocial risk factors in pregnant women of Mexican origin: The role of acculturation. American Journal of Public Health. 87(6), 10221026. 


\section{Appendix A}

\section{Letters of Permission}

1. Aspen Publishers, Inc. . . . . . . . . . . . . . 139

2. Educational Testing Service (ETS) $\ldots \ldots \ldots \ldots \ldots \ldots \ldots \ldots, 140$

3. Richard M. Suinn . . . . . . . . . . . . . . . 141

4. Vang Leng Mouanoutoua and Lillian G. Brown ........... 142

5. Harvard Program in Refugee Trauma ................. 143 


\section{Aspen Publishers, Inc. Reference Number: 9802104}

Gwendolyn Foss

676 Bancroft Drive

February 26, 1998

Rock Hill. SC 29730-3802

Dear Gwendolyn Foss:

Thank you for your interest in using our copyrighted material. PERMISSION IS GRANTED for ONE-TIME, NON-EXCLUSIVE world rights in all languages for reproduction of the msterial listed below. This permission does not extend to portions of text, charts, tables, exhibits, graphs, or photographs sourced to any other party. To use material attributed to other organizations or authors, you must seek permission directly from them. Material from U.S. Government publications is in the public domain. ELECTRONIC RIGHTS ARE NOT INCLUDED. You must include a credit line on the first page of the reproduction that includes: "Reprinted/adapted with permission from." publication. chapter/article, author(s) and/or editor(s), volume, issue, page(s), as well as, "\$ 1996 Aspen Publishers. Inc."

This letter is your invoice. Calculate the payment due and send it to the Permissions Department. You must include our Federal ID number (52-142-4553) and your Reference number 9802104.

Material(s) requested: $A N S, 19: 2, p p .74-87$

FEES: Photocopy-\$ .15 per page $x$ number of copies made

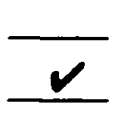
Reprint/Adapt-S 30 per page. figure, table. or exhibit Waived Please send us one complimentary copy of your publication

Payment is due 90 days from involce date

We appreciate your interest in our publications. If you need further assistance, please call (301) 417-7638.

Sincerely,

Loree Sichelstiel

Permissions Assistant

4202-5980 
NOT ICE:

The ETS Teat Collection provides micrcfiche coples of certain unpublished testa as a service to educators and psychoiontsts. It is hoped that these waterials will provide users with creative Idens for the development of their own instruments, or, in some instances, with measures of attributes for which no published tests are avallable.

The matertals included on the microfiche may be reproduced by the purchaser for his own use unt1l otherwise notifled by ETS or the author. Permission to use these matertals in any other manner must be obtalned directly from the author. This includes modifying or adapting the materlals, and selling or dlstrlbuting them to others. Any copyright notlce or cred1t lines must be reproduced exactly as provided on the origlnal.

Typlcally, the tests included in this service have not heen subjected to the intensive investigation usually associated with commercially published cests. As a consequence, inclusion of a test does not imply any judgment by ETS of the quality or usefulness of the instrument. The purchaser must assume full responsibllity for controlling access to these materials, the manner in which they are used, and the Interpretation of data derived from their application.

It $1 \mathrm{~s}$ recommended that access to these infrofiche be ifmited to staff members of professionally recognized educational and psychological Instliutions or organizations, and Individuals who are members of the Amertcan Educational Research Assoclation, the American Psychological Association, the Nattonal Counctl on Measurement in Education, or the Assoclation for Measurement-and Evaluation in Guidance. The qualifications of uthers not in these categorles should recelve careful consideration.

Finally the purchaser is urged to provide information about his use of these materials directly to the authors. Many cooperating authors are interested in collecting data on their instruments which wiII make them more useful to others. Therefore, it is to the advantage of everyone concerned - authors, present users, and users in the future - that purchasers recognize thelr professional responsibility to initlate such communication. The address of the author of this instrument as of the date on whlch this serles was released:

Mary D. Alnsworth

Department of Psycholog;

GIImer Hal1

Untversity of Virginia

Charlottesville, VA 22901

ETS Test Collection

Educational Testing Service

Princeton, New Jersey 085L 
Appendix A3

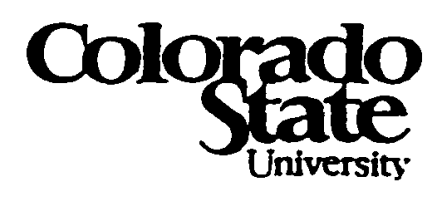

Department of Psychology

Fort Collins, Colorado 80523

(303) $491-6363$

FAX: (303) 491-1032

Dear Colleague:

You recently requested either a copy of the Suinn Lew Asian Self-Identity Acculturation Scale (SL-ASIA) or authorization to copy and use it in research.

Enclosed is a copy of the scale, and an article to be published soon that gives more data. If you are using this scale for a research study, I also give you permission, provided that: a) you cite our work in your writings, and b) you send me a summary of your findings, especially if you have means, standard deviations, norms which we can release to others.

Good luck on your project.

Sincerely.yours,

Richard M.Suinn, Ph.D.
Professor 


\title{
Hopkins Symptom Checklist-25, Hmong Version: A Screening Instrument for Psychological Distress
}

\author{
Vang Leng Mouanoutoua and Lillian G. Brown \\ Califormia School of Professional Psychology-Fresno
}

\begin{abstract}
The Hopkins Symptom Checklist (HSCL-25; Mattsson. Williams. Rickels. Lipman. \& Uhlenhuth, 1969) was translated into the Hmong language and administered to 159 Hmong adults, 73 nonclinical and 86 mental health clients. The instrument demonstrated internal consistency of .97 and had a split-half coefficient of .92 and test-retest reliability of .90 . Mental health clients produced scores that were significantly higher than those of nonclinical participants on the Anxiety. Depression, and Total scores. Consistent with expectations. Hmong more intensely affected by the casualties of war, those currently unemployed, those older, and those with less education tended to report more symptoms of anxiety and depression. The Hmong version of the HSCL-25 provided a sensitivity of $100 \%$, specificity of $78 \%$, and overall accuracy of $89 \%$, demonstrating that it is a useful screening tool for assessing general distress and anxiety problems in Hmong people.
\end{abstract}

The Hmong, mountain dwellers in Laos, experienced minimal contact with Western civilization before 1960 . They maintained a patriarchal society, practiced slash-and-burn agriculture, and believed in ancestral spirits. Their war-related involvement with the French in the 1950s and later with the American Central Intelligence Agency (C.I.A.) in the late 1960s thrust them suddenly from isolation into contemporary. Western, military, industrial society. More than $10 \%$ of their 350,000 population were killed during the 14 years of the Vietnam War (Sherman, 1988). Thousands of women were widowed, and scores of men were physically and psychologically trauma-

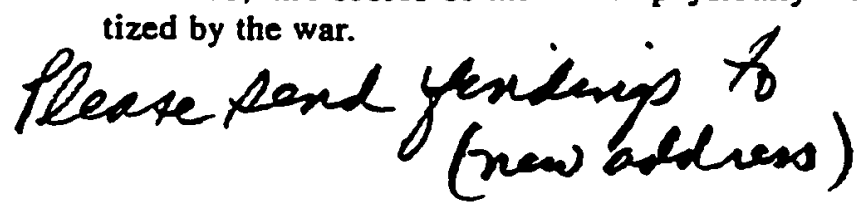

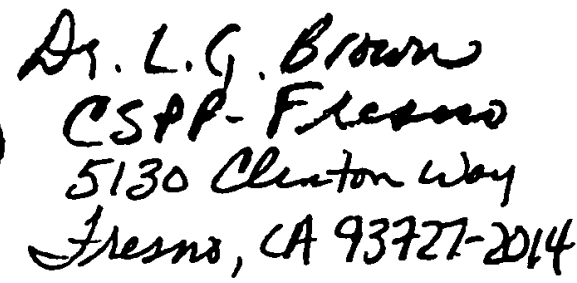


Har HARD PROGRAM IN REFUGEE TRAUMA

Riclıard F. Mollica, M.D., M.A.R., Directur

Harvard School of Public Health

Department of Health Policy and Mamagement

July 23, 1996

Gwendolyn F. Foss MSN, RN

1235 Via Barranca

La Jolla, CA 92037

Dear Ms. Foss,

This letter acknowledges receipt of $\$ 25$ for the Indochinese versions of the Hopkins Symptom Checklist-25, manual, and reprint. If you translate or modify the questionnaire we kindly request that you send us a copy of the new version for our files so that we will be able to inform other clinicians and researchers of its existence.

Best of luck with your dissertation.

Sincerely,

Keith McInnes, MS

Program Evaluator

8 Story Street, Third Floor, Cambridge, Massachusetts 02138 Tel: $617496-5550$ Fax: 617 496-5530 


\section{Appendix B}

\section{Recruitment Flyers}

1. English for Vietnamese $\ldots \ldots \ldots \ldots \ldots \ldots \ldots \ldots \ldots \ldots \ldots$

2. Vietnamese $\ldots \ldots \ldots \ldots \ldots \ldots \ldots \ldots \ldots \ldots \ldots \ldots$

3. English for Hmong $\ldots \ldots \ldots \ldots \ldots \ldots \ldots \ldots \ldots \ldots \ldots$

4. Hmong $\ldots \ldots \ldots \ldots \ldots \ldots \ldots \ldots \ldots \ldots \ldots \ldots$ 
Appendix B 1

Date: April 27, 1997

\section{To mothers of babies:}

My name is Gwendolyn F. Foss. I am a nurse researcher at the University of San Diego. 1 would like to learn more about how mothers, fathers, and families care for their babies who are between one week and twelve months old. I would like to visit you and your baby two times in your home. If you agree. each visit would last about one and a half hours. During these home visits, I would like to ask you some questions about how you may or may not have changed since coming to the United States. I would like to ask you how you care for your baby now, compared to how you cared for your baby in Vietnam. I would also like to ask you about problems you may or may not be experiencing since you came to the United States. I would also like to weigh. and measure the growth and development of your baby. These are procedures commonly done by nurses in clinics and in homes.

If you are interested in learning more about this study, or wish to be part of it, please call me at 459-6503. Thank you for considering participating in my study.

Gwendolyn F. Foss, RN PHN MSN

Doctoral Candidate, University of San Diego

459-6503 
San Diego ngày ___ tháng__ năm

Kính gơi: Các Bà Mẹ có con nhỏ

Thưa Qưi Bà,

Gwendolyn Foss, chuyên viên nghiên cứu. Nghiên cứu sự nuôi dưỡng trè em cưa các Bà Mẹ Việt-Nam và Laò, đã địnhcu tại Hoa Kỳ trong vòng 20 năm qua. Nếu có thế được , xin Qúi Bà cho phép tôi : được đến nhà hai lần, mỗi lần khoảng một giờ rưỡi. dế quan sát và học hỏi thêm về phương pháp nuôi dưỡng và cách săn sóc tré em tuối từ một tuần đến haî tuoổi rưỡi của Qưi Bà và gia đình. Đồng thời tôi cūng rất muốn biết về cách nuôi dưỡng và săn sóc tré sơ sinh của Qưi Bà ở Việt-Nam và sau khi định cư tại Hoa Kỳ. Nếu có sự khác biệt nào về phương pháp nuôi dưỡng tré em, xin Qúi Bà vui lòng cho tôi biết. Tôi cūng muốn chia xẻ, trao đối kinh nghiệm cùng Qưi Bà về những trở ngại, nhūnng khó khăn khi Qưi Bà định cư tại Hoa Kỳ. Sau cùng, xin Qưi Bà cho tôi biết trọng lượng, chiều cao và chu vi đầu cưa em bé để theo dói sự tăng trưởng của em bé.

Nếu Qưi Bà muốn tìm hiểu thêm, hoặc muốn tham gia vào chương trình nghiên cưu nầy, xin Qúi Bà vui lòng liên lạc tôi ở số 459-6503.

Xin chân thành cảm ơn Qưi Bà dã cho tôi cơ hội nghiên cứu và học hỏi nầy.

Gwendolyn F. Foss

Ứng Viên Tiến Sĩ Điều Dưỡng

Viện Đại Học San Diego

Diện thoại: 459-6503 
Date:

To mothers of babies:

My name is Gwendolyn F. Foss. I am a nurse researcher at the University of San Diego. I would like to learn more about how mothers, fathers, and families care for their babies who are between one week and eleven months old. I would like to visit you and your baby in your home. If you agree, each visit would last about one and a half hours. During these home visits, I would like to ask you some questions about how you may or may not have changed since coming to the United States. I would like to ask you how you care for your baby now, compared to how you cared for your baby in Laos. I would also like to ask you about problems you may or may not be experiencing since you came to the United States. Lastly, I would like to weigh, and measure the growth and development of your baby. These are procedures commonly done by nurses in clinics and in homes.

If you are interested in learning more about this study, or wish to be part of it, please call me at 459-6503, or leave your name with

Thank you for considering participating in my study.

Gwendolyn F. Foss, RN PHN MSN

Doctoral Candidate, University of San Diego 
Nyob zoo rau cov niam muaj menyuam mos liab:

Kuv lub npe hu ua Gwendolyn F. Foss. Kuv yog ib tus hwjtxiv neeb kawm ntawv nras lub tsev ntawv University of San Diego. Kuv xav paub txog cov niam txiv los yog cov tsev neeg muaj menyuam mos liab hnub nyoog ib asthiv txog lis kaum ib hli tej ntawd. Yog nej muaj sijhawm, kuv xav tuaj sib ntsib nrog nej thiab nej tus menyuam tom nej tsev, piv xas lis ib xuas moos tej. Kuv xav noog saib pib li thaum nej tuaj nyob tebchaws Asmeslivkas no, nej cov kevcai saib xyuas nej cov menyuam mos liab pauv txawv npaum li cas thaum nej nyob tebchaws Los Tsuas. Tias kuv xav noog saib nej muaj lo tsis muaj teeb meem loj thaum nej tuaj nyob tebchaws Asmeslivkas no. Thaum kawg, kuv xav ntsuas thiab luj saib nej tus menyuam mos liab muaj ceeb thawj npaum li cas. Cov no yog ib cov kevcai qhuaj raws li tom tsev qhuaj mob tiab xwb.

Yog nej xav paub ntxiv, thov hu kuv tus xov tooj: $459-6503$, los tseg nej lub npe nrog

Ua tsuag ntau,

Givendolyn F. Foss, RN PHN MSN

Doctoral Candidate, University of San Diego 


\section{Appendix C}

\section{Consent for Participation}

1. English ....................... 150

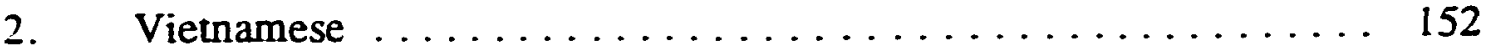

3. Hmong ......................... 154 


\section{CONSENT FOR PARTICIPATION}

Gwendolyn F. Foss is a doctoral student in the College of Nursing at the University of San Diego. She is conducting a research study about mothers who have moved to the United States from Laos or Vietnam within the last 22 years, and how they and their families care for their infants since arriving in the USA. After giving consent to participate in the study, I understand that I will be visited two times in my home for about one and one half hours. The two home visits will be one to four weeks apart.

During the two home visits, I understand that I will be asked for a short health history of myself and my baby. I understand that Mrs. Foss will weigh and measure the length and head circumference of my baby, and measure the development of my baby, using the Denver Developmental Screening Test. I also understand that Mrs. Foss will observe me and my family taking care of my baby and and will ask about how we care for my baby. I understand that I will be asked to complete a questionnaire about possible symptoms or problems people sometimes have, and about how much I do or do not feel like an American. I understand that either I or Mrs. Foss may provide an interpreter if I choose to use one.

Participation in the study should not involve any added risks or discomforts to me except for possible minor fatigue. I understand that my participation may help nurses who work with immigrants to understand how both traditional customs and American customs are used in the care of infants after immigrants settle in this country.

I understand my research records will be kept completely confidential. My identity will not be disclosed without consent as required by law. I further understand that to preserve my anonymity only group data will be used in any publication of the results of this study.

My participation in this study is entirely voluntary. I understand I may refuse to participate or withdraw at any time. I understand that if Mrs. Foss observes neglectful or abusive behavior toward any child in the house she is required by law to inform the Child Protective Services.

Gwendolyn F. Foss has explained this study to me and answered my questions. If I have other questions or research-related problems, I can reach Ms. Foss or leave a message for her at $459-6503$.

There are no other agreements, written or verbal, related to this study beyond that expressed on this consent form. 
I, the undersigned, understand the above explanations and, on that basis I give consent to my voluntary participation in this research.

Signature of Mother or Parent/Guardian

Date

Location

Signature of Principal Researcher

Date

Signature of Witness

Date 


\section{Bản Hợp Dồng}

Bà Gwendolyn F. Foss chuyên viên nghiên cứu sự nuôi dưỡng trẻ em thuộc Viện Đại học San Diego. ba đăng thực hiện việc nghiên cứu vấn đề nuôi dưỡng các em bé từ lúc mới sinh đến 11 tháng tuới của các bà mẹ Việtnam và Lào đã định cu tại Hoa Kỳ trong vòng hai năm qua.

Sau khi chấp nhận tham gia chương trình, tôi đồng ý cho phép bà Foss đến nhà tôi hai lần, cách nhau hai đến bốn tuần lễ. Mỗi lần khoảng một giờ rưởi. Trong hai lần thăm viếng đó , tôi đồng ý cho bà biết về sức khỏe tổng quát của tôi và em bé cho bà biết trong lượng, chiều cao và chu vi đầu của em bé, và theo dói sự tăng trưởng của em bé dựa theo Bản Khảo Sát Sự Tăng Trưởng cưa chương trình Denver.Tôi cūng được biết bà sẽ quan sát tìm hiểu về phương pháp nuôi dưỡng và săn sóc em bé của tôi và gia đình tôi. Tôi sē trả lời bà những câu hỏi liên quan đến triệu chứng, những khó khăn thường gặp, những cảm giác nào mà tôi cảm thấy giống hoặc không giống như người Mỹ.

Tôi được biết sự tham gia vào chương trình cūa tôi sē giúp các $Y$ tá hiếu rõ phong tục, tập quán cũa các di dân hầu giúp họ dể dàng làm viiệc với các bà mẹ vừa định cư tại xư sở này. Sụ tham gia của tôi vào chương trình hoàn toàn tình nguyện vì sû0 cần thiết và lợi îch của nó, cūng như không gây khó khăn và trở ngại cho tôi và gia dình tôi. Tôi có quyền tư chối hoạc ngưng tham gia vào chương trình này bất cứ lúc nào tôi muốn.

Tôi được biết hồ sơ nghiên cưu sẽ được giữ kín. Danh tánh của tôi sẽ khônh được tiết lộ nếu không có sự đồng ý của tôi. Nói cách khác, chỉ những dử kiện của cuộc khảo cứu có thế được sử dụng trong việc phố biến các kết qửa của cuộc nghiên cứu mà thôi. Tôi cũng hiếu rằng nếu tôi cố tình với ác ý không sân sóc, nuôi đưỡng con tôi theo luật địng, thì buộc bà phải báo cáo cho cơ quan liên hệ. Bà Foss hoặc tôi có thể nhờ thông dịch viên nếu chưng tôi cần đến sự giưp đở của họ.

Bà Gwendolyn F. Foss đā giải thích và đã trả lơì thóa đáng mọi thắc măc của tôi. Nếu tôi có những thăc mắc khác tôi có thể liên lạc với bà ở số điện thoại 459-6503.

Tôi xác nhận không có những thỏa thuận bằng miệng hoặc bằng giấy tờ naò khác ngoại trừ những điều đã trưng dẩn ở bản hợp đồng nầy.

Tôi ký tên dưới đây xác nhận đã hiểu rō tất cả những điều thỏa thuận đā nói ở phần trên. Trên căn bản đó, tôi đồng ý tự nguyện tham gia vào chương trình nghiên cứu nầy. 
Nơi Ký

Chử ký của Chuyên viên Nghiên Cứu

Ngày Ký

Chử ký cưa Nhân Chứng

Ngày Ký 
Gwendolyn F. Foss yg ib tus hwjtxiv neeb kawm ntawv nras lub tsev ntawv Universityh of san Diego. Nws sav kawm txog cov niam ua tsiv tebchaws Los Tsuas thiab tebchaws Nyablaj tuaj raus tim Asmeslivkas teb no tau nees nkaum thawv xyoos no, thiab nws sav paub saib cov niam tau tu lawv cov menyuam mos liab zoo li cas. Kuv xav pab nws kawm txog kuv thiab kuv tus menyuam. kuv paub tia nws yuav tuaj nrog kuv sib ntsib ob zaus. ob lub sijhawm ntawd yuav kas li ib xyuas txog ib xyuas moos tawm.

Thaum nws tuaj ntsib kuv, kuv paub tias nws yuav noog txog kuv thiab kuv tus menyuam kev muaj mob tas los lawm. Kuv paub tia tus hwjtxiv neeb Foss yuav siv lub ntsuas thiab lub luj menyuam hu ua Denver Developmental Screening Test no los ntsuas thiab luj kuv tus menyuam mos liab. Kuv paub tias tus hwjtxiv neeb Foss yuav saib thiab noog txog tej kevcai zov tiab tu txog kuv tus menyuam mos liab. Kuv paub tias kuv yuav tau teb txog ob peb daim ntawv nws noog txog kev teeb meem, thiab kev uas neej nyob tebchaws Asmeslivkas no. Kuv paub tias yog tus hwjtxiv neeb Foss pom kuv ntaus, los yog cia kuv cov menyuam yooj tshaib hauv kuv lus tsev, nes yuav muaj xiv hu mus qhia rau nrad cag tsev saib xyuas menyuam hu ua Child Protective Services no. Kuv paub tias kuv los yog tus hwjtxiv neeb Foss ma lis nrhiav ib tug neeg txhais lus rau kuv.

Kuv paub tias yog kuv pab ua qhov haujlwm no, kuv yuav muaj tej lub caij sifhawm ua rau kuv nkees tiab. Tiamsis mas kuv yuav pab cov hwjtxiv neeb kom lawv tias li pab tau cov tib neeg pluag tsiv tuaj rau Asmeslivkas tebshaws no, kom lawv tias li paub tias peb zov menuam txawv lis cas.

Kuv paub tias kuv muab kuv lub sijhawm dawb pab tus hwjtxiv neeb no xwb. Hnubtwg kuv xav tawm cov haujlwm no los tau.

Kuv paub tias yog kuv tsis kam tso lus, kuv lub npe pab qhov haujlwm no yuav tsis tawm rau lwm tus neeg siv lis.

Gwendolyn F. Foss qhaiv tas nws cov ntawv thiab pab kuv to taub zaj haujlwm no lawm. Yog kuv xav paub ab tsi ntxiv kuv ma lis ju hwjtxiv neeb Foss tom nws tsev, 459-6503.

Ntawv thiab lus cog cia lis nov lawm $\mathrm{xwb}$. 
Kuv sau kuv lub npe cog cia daim ntawv nov hais tias kuv to taub txog txoj haujlwm nov, tias kuv muab kuv lub sijhawm pab dawb $\mathrm{xwb}$.

Tus Niam Lub Npe

Qhovchaw Nyob

Tus Hwjtxiv Neeb Lub Npe

Tus Povthawj Lus Npe
Hli ntuj

Hli ntuj

Hli Ntuj

Hli ntuj 
Appendix D

Demographic and Misc. Data $\ldots \ldots \ldots \ldots \ldots \ldots \ldots \ldots \ldots \ldots$ 
Appendix D

\section{DEMOGRAPHIC AND MISC DATA}

1. How old are you ?

How old is your husband $?$

How old are your children $?$

2. What was your occupation in your homeland What is your occupation here in San Diego

3. What was your husband's occupation in his homeland What is your husband's occupation here in San Diego ? ?

4. How old were you when you left your homeland ? How old were you when you arrived in the United States $?$

5. What was the highest grade you completed in your homeland What is the highest grade you completed in the USA ?

6. What was the highest grade your husband completed in your homeland $?$ What is the highest grade your husband completed in the USA ?

7. Who made the decision to leave your homeland

8. What was the reason for leaving your homeland

9. How many of your own or your husband's family members live in the United States ?

10. How many of your family members still live in your homeland $?$

11. How many times have you been pregnant How many children have been born to you How many miscarriages have you had How many of your babies were born dead ?

How many of your babies died before the age of one year

12. How much did this baby weigh when he/she was born How long was this baby when he/she was born ? ? 
18. How is the information you were given by your doctor or nurse different from your family's (you or your parents) usual practices?

19. Which family members help you provide care for your baby during the first year?

20. What does each do?

21. What are the things you do to keep your baby from getting sick?

22. What are the most important things your baby needs to learn about your culture and about the American culture by the time she or he starts first grade?

23. How has moving to the United Stated changed how you and your family care for your baby (such as who helps you care for your baby, what kinds of things you do for your baby, what you feed your baby etc.)?

24. Is there anything else you think I should know? 


\section{Appendix E}

Sensitivity Versus Insensitivity to the Baby's Communications Scale with Scale

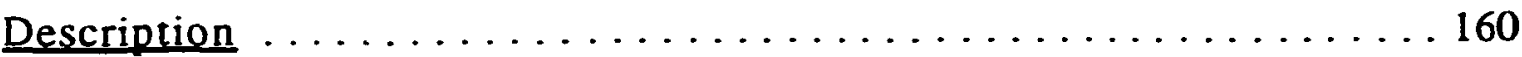




\section{MATERNALSENSITIVITY SCALE}

(Ainsworth)

Mother ID

Visit \# 1 Visit \# 2

Age of infant___ Age of mother

Date of Home Visit

Time of Home Visit.

Sensitivity vs. Insensitivity

\begin{tabular}{lllllllll}
\hline 1 & 2 & 3 & 4 & 5 & 6 & 7 & 8 & 9
\end{tabular}


AINSWORTH SCALES - 1977

\title{
SENSITIVITY VERSUS INSENSITIVITY TO THE BABY'S COMMUNICATIONS
}

\author{
SCALE DESCRIPTION
}

This variable deals with the mother's ability to perceive and to interpret accurately the signals and communications implicit in her infant's behavior, and given this understanding, to respond to them appropriately and promptly. Thus the mother's sensitivity has four essential components:

(a) her awareness of the signals:

(b) an accurate interpretation of them;

(c) an appropriate response to them; and

(d) a prompt response to them.

Let us consider each of these in tum.

The mother's awareness of her baby's signais and communications has two aspects. The first is the same as the issue covered in the scale "accessibility versus ignoring and neglecting." In other words, the mother must be reasonably accessible to the baby's communications before she can be sensitive to them. Accessibility is a necessary condition for sensitive awareness. It is not a sufficient condition, however, for a mother can maintain the "baby" in her field of awareness without fulfilling the other condition for sensitive awareness. The second aspect of awareness may be described in terms of "thresholds." The most sensitive mother-the one with the lowest threshold-is alert to the baby's most subtle, minimal, understate cues. Mothers with higher thresholds seem to perceive only the most blatant and obvious communications. Mothers with the highest thresholds seem often oblivious, and are, in effect, highly inaccessible. This second aspect is very closely related to the question of interpretation of the baby's signals, for usually the mother who is alert to minimal cues also interprets them correctly. This is not invariably the case, however. For example, some mothers are alert to the slightest mouth movements, and sometimes incorrectly interpret them as hunger-or they notice minimal tensions or restlessness and incorrectly interpret them as fatigue.

The mother's ability to interpret accurately her baby's communications has three main components (a) her awareness, as previously discussed; (b) her freedom from distortion, and (c) her empathy. An inattentive, "ignoring" mother is, of course, often unable to interpret correctly the baby's signals when they break through her obliviousness, for she has been unaware of the prodromal signs and of the temporal context of the behavior. But even a mother who is highly aware and accessible may misinterpret signals because her perception is distorted by projection, denial, or othermarked defensive operations. Mothers who have distorted perceptions tend to bias their "reading" of their babies according to their own wishes, moods, and fantasies. For example, a mother not wishing to attend to her baby might interpret his fussy bids for attention as fatigue and, therefore, put him to bed; she being in a hurry, might perceive any slowing down in the rate of feeding as a sign of satiation; or a mother who is somewhat rejecting of her infant might perceive him as rejecting and aggressive towards herself. Mothers who least distort their perceptions of their babies have some insight as to their own wishes and moods, and thus can more realistically judge the baby's behavior. Furthermore, they are usually aware of how their own behavior and moods affect their infant's behavior.

The mother must be able to empathize with her baby's feelings and wishes before she can respond with sensitivity. That is, a mother might be quite aware of and understand 
accurately the baby's behavior and the circumstances leading to her baby's distress or demands. but because she is unable to empathize with him-unable to see things from the baby's point of view--she may tease him back into good humor, mock him, laugh at him, or just ignore him. The mother's ego-centricity and lack of empathy may also lead to detached intellectual responses to the baby rather than to warm, sensitive interactions with the baby.

A high threshold of awareness and inaccurate perceptions certainly lead to insensitive responses. Nevertheless, the mother may be highly aware and accurate in her interpretation and still be insensitive. Therefore, in the last analysis, the appropriateness and promptness of the mother's response to communications are the hallmarks of sensitivity.

The quality of the mother's interaction with her infant is probably the most important index of her sensitivity. It is essential that the mother's responses be appropriate to the situation and to the baby's communications. Often enough, at least in the first year of life, the sensitive mother gives the baby what his communications suggest he wants. She responds socially to his attempts to initiate social interaction, playfully to his attempts to initiate play. She picks him up when he seems to wish it, and puts him down when he wants to explore. When he is distressed, she knows what kind and degree of soothing he requires to comfort him-and she knows that sometimes a few words or a distraction will be all that is needed. When he is hungry she sees that he soon gets something to eat, perhaps giving him a snack if she does not want to give him his regular meal right away. On the other hand, the mother who responds inappropriately tries to socialize with the baby when he is hungry, play with him when he is tired, or feed him when he is trying to initiate social interaction.

In play and social interaction, the mother who responds appropriately to her child does not overstimulate him by interacting in too intense, too vigorous, too prolonged, or too exciting a manner. She can perceive and accuraiely interpret the signs of overexcitement, undue tension, or incipient distress and shifts the tempo or intensity before things have gone too far. Similarly, she is unlikely to understimulate the child, because she picks up and responds to the signals he gives when he is bored or when he wants more interaction than has heretofore been forthcoming.

In the second year of life, and sometimes also toward the end of the first year, it is maximally appropriate for the mother to respond to the baby's signals not so much in accordance with what he ostensibly wants as in terms of a compromise between this and what will make him feel most secure, competent, comfortable, etc. in the long run. This is a tricky judgment to make, for so much that is done "for the baby's own good" is done both contrary to his wishes and according to the mother's convenience, whim, or preconceived standards. Nevertheless there are situations in which limit-setting, even in the first year, clears the air even though it is initially contrary to the baby's wishes. Similariy there are situations in which the baby's signals might lead the mother to increase the tempo of interactions to the point of discomfort for him, and in which it is appropriate gradually to diminish intensity. Furthermore, there is a fine point of balance at which the mother can begin to show the baby that she is not an instrument of his will, but a cooperative partner whose participation must be elicited appropriately. In such instances the mother will slightly frustrate the baby's imperious demands but warmly encourage (and reward) behaviors which are inviting or requesting rather than demanding. Nevertheless in such interactions the sensitive mother acknowledges the baby's wishes even though she does not unconditionally accede to them. The chief point is that a sensitive, appropriate response does not invariably imply complete compliance to the baby's wish-although very frequently compliance may be the most appropriate response. 
The final feature of appropriate interaction is that it is well-resolved, or wellrounded and completed. For example, when the baby seeks contact, the sensitive mother holds him long enough to satisfy him, so that when he is put down he does not immediately seek to be picked up again. When he needs soothing, she soothes him thoroughly, so he is quite recovered and cheerful. When he seeks social interaction she enters into a more or less prolonged exchange with him, after which, often encugh, he is content to entertain himself. In contrast, some mothers with low sensitivity seem to be fragmented and incomplete, these mothers may try a series of interventions as though searching for the best method or solution. Highly sensitive mothers have completed, easily and well resolved interactions.

Finally, there is the issue of the promptness of the mother's response to the baby's communication. A response, however appropriate, which is so delayed that it cannot be perceived by the baby as contingent upon his communication cannot be linked by him to his own signal. On the assumption that it is a good thing for a baby to gain some feeling of efficacy-and eventually to feel cumulatively a "sense of competence" in controlling his social environment-it seems a part of sensitivity to acknowledge the baby's signals in some effective way and to indicate that one is at least preparing to accede to them. During the first quarter of the first year, a mother's prompt response to the baby's social communication and signals is probably a more critical measure. A mother is inevitably insensitive when she fails to respond to the baby's outstretched arms, to his excited greeting, or simply to his smile or gentle touch.

An issue which cuts across the various components of sensitivity concerns the timing of routine activities and play. In general, arbitrary or very rigid timing of major interactions cannot but be insensitive to the infant's signals, moods, and thythms. The mother who arranges and organizes day by day activities with her infant in order to most convenience herself, or the mother who thinks by the clock, has little or no consideration of the infant's tempo and current state.

In summary, highly sensitive mothers are usually accessible to their infants and are aware of even their more subtle communications, signals, wishes, and moods; in addition, these mothers accurately interpret their perceptions and show empathy with their infants. The sensitive mother, armed with this understanding and empathy, can time her interactions well and deals with her baby so that her interactions seem appropriate--appropriate in kind as well as in quality-and prompt. In contrast, mothers with low sensitivity are not aware of much of their infant's behavior either because they ignore the baby or they fail to perceive in his activity the more subtle and hard-to-detect communications. Furthermore, insensitive mothers often do not understated those aspects of their infant's behavior of which they are aware of else they distort it. A mother may have somewhat accurate perceptions of her infant's activity and moods but may be unable to empathize with him. Through either lack of understanding or empathy, mothers with low sensitivity improperly time their responses, either in terms of scheduling or in terms of promptness to the baby's communications. Further, mothers with low sensitivity often have inappropriate responses in kind. as well as quantity, i.e. interactions which are fragmented and poorly resolved. 
AINSWORTH SCALES - 1977

\title{
SENSITIVITY VERSUS INSENSITIVITY TO THE BABY'S COMMUNICATIONS
}

\author{
SCALE CODES
}

9 Highly sensitive. This mother is exquisitely attuned to B's signals, and responds to them promptly and appropriately. She is able to see from B's point of view; her perceptions of his signals and communications are not distorted by her own needs and defenses. She "reads" B's signals and communications skillfully, and knows what the meaning is of even his subtle, minimal, and understated cues. She nearly always gives $B$ what he indicates that he wants, although perhaps not invariably so. When she feels that is best not to comply with his demands-for example, when he is too excited, over-imperious, or wants something he should not have-she is tactful in acknowledging his communication and in offering an acceptable altemative. She has "well-rounded" interactions with B, so that the transaction is smoothly completed and both she and B feel satisfied. Finally, she makers her responses temporally contingent upon B's signals and communications.

7. Sensitive. This mother also interprets B's communications accurately, and responds to them promptly and appropriately--but with less sensitivity than mothers with higher ratings. She may be less attuned to B's more subtle behaviors than the highly sensitive mother. Or, perhaps because she is less skillful in dividing her attention between B and competing demands, she may sometimes "miss her cues." B's clear and definite signals are, however, neither missed nor misinterpreted. This mother empathizes with $B$ and sees things from his point of view; her perceptions of his behavior are not distorted. Perhaps because her perception is less sensitive than that of mothers with higher ratings, her responses are not as consistently prompt or as finely appropriate--but although there may be occasionally little "mismatches", M's interventions and interactions are never seriously out of tune with B's tempo, state and communications.

5. Inconsistently sensitive. Although this mother can be quite sensitive on occasion, there are some periods in which she is insensitive to B's communications. M's inconsistent sensitivity may occur for any one of several reasons, but the outcome is that she seems to have lacunae in regard to her sensitive dealings with B--being sensitive at some times or in respect to some aspects of his experience, but not in others. Her awareness of B may be intermittent-often fairly keen, but sometimes impervious. Or her perception of B's behavior may be distorted in regard to one or two aspects although it is accurate in other important aspects. She may be prompt and appropriate in response to his communications at some times and in most respects, but either inappropriate or slow at other times and in other respects. On the whole, however, she is more frequently sensitive than insensitive. What is striking is that a mother who can be as sensitive as she is on so many occasions can be so insensitive on other occasions. 
3. Insensitive. This mother frequently fails to respond to B's communications appropriately and/or promptly, although she may on some occasions show capacity for sensitivity in her responses to and interactions with $B$. Her insensitivity seems linked to inability to see things from B's point of view. She may be too frequently preoccupied with other things and therefore inaccessible to his signals and communications, or she may misperceive his signals and interpret them inaccurately because of her own wishes or defenses, or she may know well enough what $B$ is communicating but be disinclined to give him what he wants--because it is inconvenient or she is not in the mood for it, or because she is determined not to "spoil" him. She may delay an otherwise appropriate response to such an extent that it is no longer contingent upon his signal, and indeed perhaps is no longer appropriate to his state, mood, or activity. Or she may respond with seeming appropriateness to B's communications but break off the transactions before $B$ is satisfied, so that their interactions seem fragmented and incomplete or her responses perfunctory, half-hearted, or impatient. Despite such clear evidence of insensitivity, however, this mother is not as consistently or pervasively insensitive as mothers with even lower ratings. Therefore when the baby's own wishes. moods, and activity are not too deviant from the mother's wishes, moods, and household responsibilities or when the baby is truly distressed or otherwise very forceful and compelling in his communication, this mother can modify her own behavior and goals and, at this time, can show some sensitivity in her handling of the child.

1. Highly insensitive. The extremely insensitive mother seems geared almost exclusively to her own wishes, moods, and activity. That is, $M$ 's interventions and initiations of interaction are prompted or shaped largely by signals within herself; if they mesh with B's signals, this in often no more that coincidence. This is not to say that $M$ never responds to B's signals; for sometimes she does if the signals are intense enough, prolonged enough or often enough repeated. The delay in response is in itself insensitive. Furthermore, since there is usually a disparity between M's own wishes and activity and B's signals, $M$ who is geared largely to her own signals routinely ignores or distorts the meaning of $B$ 's behavior. Thus, when $M$ responds to B's signals, her response is characteristically inappropriate in kind or fragmented and incomplete. 


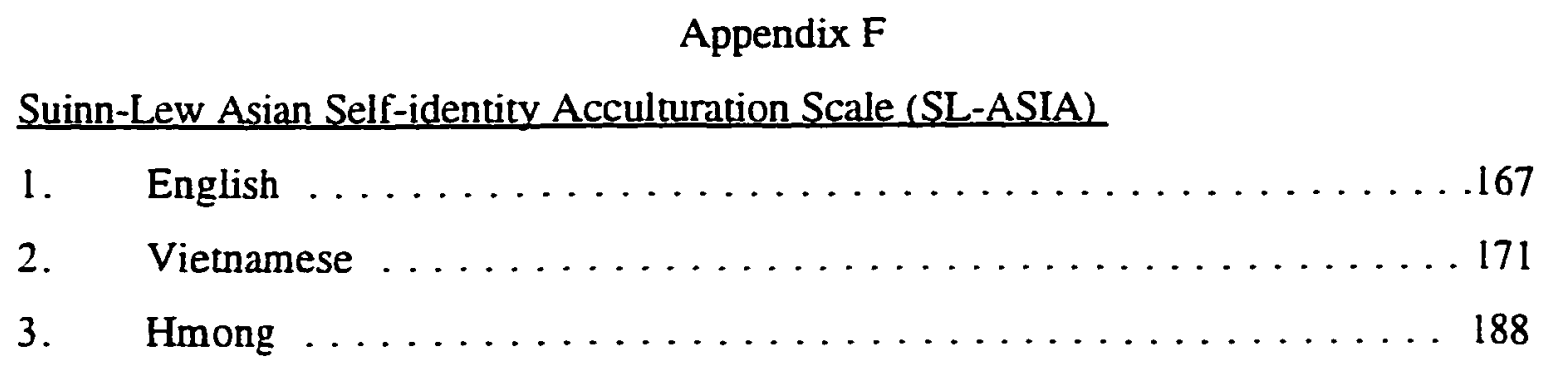


INSTRUCTIONS: The questions which follow are for the purpose of collecting inforwation about your historical background as well as more recent behaviors which may be related to your cultural identity. Choose the one answer which best describes you.

1. What language can you speak?

I. Asian only (for example, Chinese, Japanese, Korean, Vietnamese, etc.)

2. Mostly Asian, some English

3. Astan and English about equally well (bilingual)

4. Mostly English, some Asian

5. Only English

2. What language do you prefer?

1. Astan only (for example, Chinese, Japanese, Korean, Vletnamese, etc.)

2. Mostly Asian, some English

3. Astan and English about equally well (bilingual)

4. Mostly English, some Astan

5. Only English

3. How do you identify yourself?

1. Oriental

2. Astan

3. As 1an-American

4. Chinese-American, Japanese-American, Korean-American, etc.

5. American

4. Which Identification does (did) your mother use?

1. Oriental

2. Asian

3. Asian-American

4. Chinese-American, Japanese-American, Korean-American, etc.

5. American

5. Which identification does (did) your father use?

1. Ortental

2. Aslan

3. Astan-American

4. Chinese-Awertcan, Japanese-American, Korean-Awerican, etc.

5. Amertcan

6. What was the ethnic origin of the friends and peers you had, as a child up to age 6 ?

1. Almost exclusively Astans, Astan-Americans, urlentals

2. Mostly Asians, Aslan-Americans, Orfentals

3. About equally Astan groups and Anglo gruups

4. Mostly Anglos, Blacks, Hispanics, or orher non-Astan ethnic groups

5. Almost exclusively Anglos, Blacics, Hispanirs or other non-Aslan ethnic groups

7. What was the ethnic orlgin of the friends and peers you had, as a chlld from 6 to 18 ?

1. Almost exclusively Astans, Astan-Amertedns, Urlentals

2. Mostly Asians, Asian-Americans, Orlentals

3. About equally Aslan groups and Anglo groups

4. Mostly Anglos, Blacks, Hispanies, or orher nun-Aslan ethnic groups

5. Almost exclusively Anglos, Blacks, Hispanics or other non-Asian ethnic groups

8. Whom do you now assoclate with in the communtey?

1. Almost exclusively Asians, Asian-Americans, Urlentals

2. Mostly Aslans, Aslan-Americans, Orientals

3. About equally Astan groups and Anglo groups

4. Mostly Anglos, Blacks, Hispanics, or other non-Aslan ethnic groups

5. Almost exclusively Anglos, Blacks, Hispantes or other non-Asian ethntc groups 
Page 2

9. If you could pick, whom would you prefer to associate with in the community?

1. Almost exclusively Asians, Asian-Americans, Orientals

2. Mostly Asians, Asian-Awericans, Orientals

3. About equally Asian groups and Anglo groups

4. Mostly Anglos, Blacks, Hispanies, or other non-Asian ettnic groups

5. Almost exclusively Anglos, Blacks, Hispanics, or other non-Asian ethnic groups

10. What is your music preference?

1. Only Astan music (for example, Chinese, Japanese, Korean, Vietnamese, etc.)

2. Mostly Astan

3. Equally Asian and English

4. Mostly English

5. English only

11. What is your movie preference?

1. Astan-language movies only

2. Astan-language movies mostly

3. Equally Asian/English

4. English-language movies mostly

5. English-language movies only

12. Where were you born?

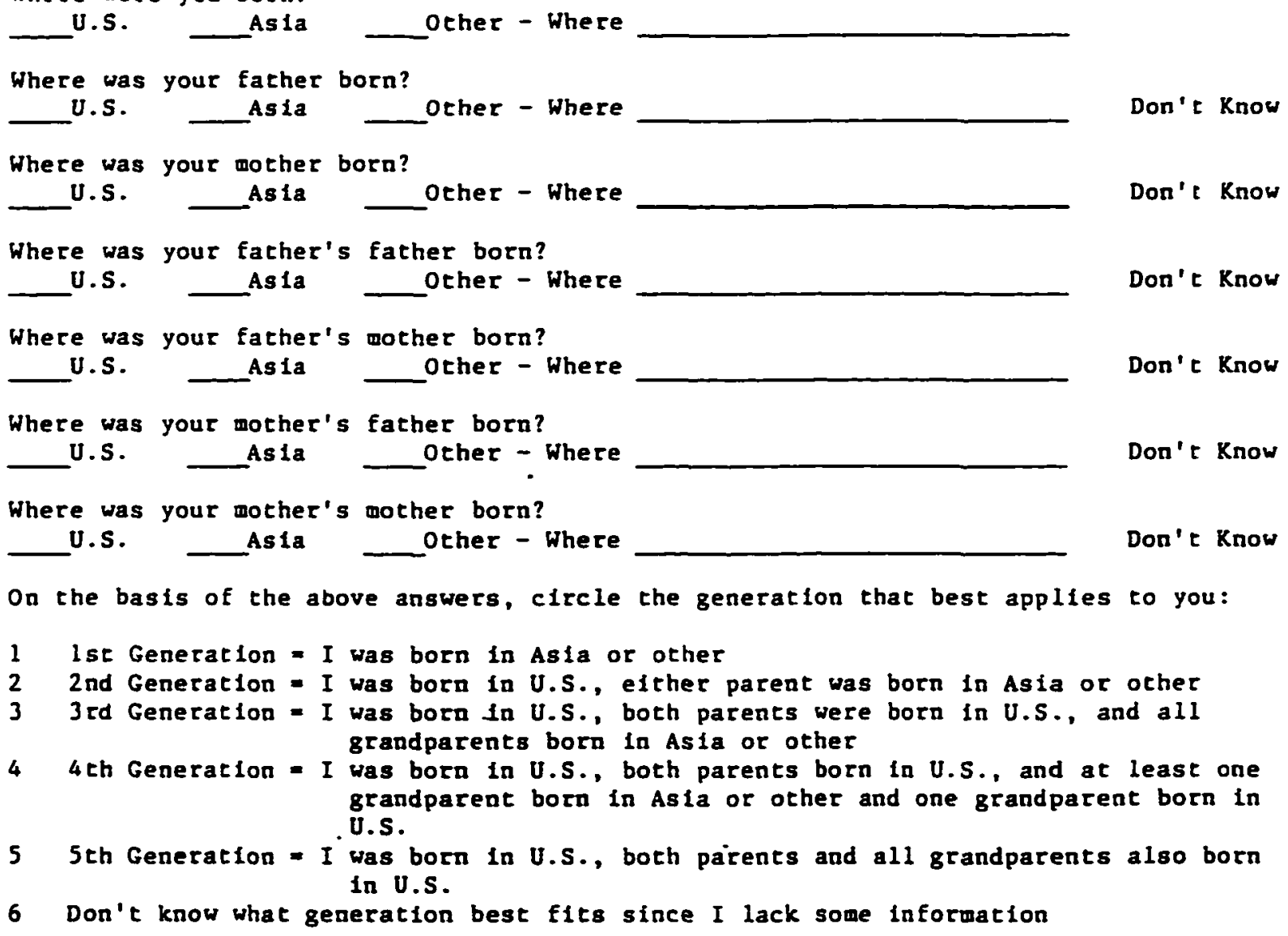

13. Where were you rafsed?

1. In Asla only

2. Mostly in Asta, some in U.S.

3. Equally in Asia and U.S.

4. Mostly in U.S., some In Asia

5. In II.S. nnlv 
SL-AS LA

Page 3

14. What contact have you had with isia?

i. Ralsed one year or more in Asia

2. Lived for less than one year in Asia

3. Occasional visits to Asta

4. Occasional communicatlons (letters, phone calis, etc.) with peopie in Asia

5. No exposure or communications with people in Asta

15. What is your food preference at home?

1. Exclusively Asian food

2. Mostly Asian food, some American

3. About equally Asian and American

4. Mostly American food

5. Exclustuely American food

16. What is your food preference in restaurants?

1. Exclusively Astan food

2. Mostly Aslan food, some American

3. About equaliy Astan and American

4. Mostly American food

5. Exclusively American food

17. Do you

1. read only an Aslan language

2. read an Asian language betcer than English

3. read both Astan and English equally well

4. read English betcer than an Asian language

5. read only English

18. Do you

1. write only an Astan language

2. write an Asian language better than English

3. write both Asian and English equaliy well

4. write English better than an Asian language

S. write only English

19. If you consider yourself a member of the Astan group (Oriental, Astan, Astan-American, Chinese-American, etc., whatever term you prejer), how wuch pride do you have in this group?

1. Extremely proud

2. Moderately proud

3. Litrle pride

4. No pride but do not feel negative toward group

5. No pride but do feel negat Ive toward group

20. How would you rate yourself?

1. Very Astan

2. Mostly Astan

3. Bicultural

4. Mostly Westernized

5. Very Hesternized

21. Do you participate in Aslan occasions, holidays, traditions, etc.?

1. Nearly all

2. Most of them

3. Some of them

4. A few of them

5. None at all 
SL-ASIA

Page 4

22. Rate yourself on how much you believe in Asian values (e.g., about marriage, families, education, work):
1
2
3
4
(do not believe)
(strongly believe in Asian values)

23. Rate your self on how much you believe in American (Western) values:

$\begin{array}{lllll}1 & 2 & 3 & 4 & 5 \\ \text { (do not believe) } & & & & \text { (strongly believe in American values) }\end{array}$

24. Rate yourself on how well you fit when with other Asians of the same ethricity: $\begin{array}{lllll}1 & 2 & 3 & 4 & 5 \\ \text { (do not fit) } & & & & \text { (fit very well) }\end{array}$

25. Rate yourself on how well you fit when with other Americans who are non-Asian (Westerners):

$\begin{array}{llllll}1 & 2 & 3 & 4 & 5\end{array}$

(do not fit) (fit very well)

26. There are many different ways in which people think of themselves. Which ONE of the following most closely describes how you view yourself?

1. I consider myself basically an Asian person (e.g., Chinese, Japanese, Korean, Vietnamese, etc.). Even though I live and work in America, I still view myself basically as an Asian person

2. I consider myself basically as an American. Even though I have an Asian background and characteristics, I still view myself basically as an American.

3. I consider myself as an Asian-American although deep down I always know I am an Asian.

4. I consider myself as an Asian-American, although deep down, I view myself as an American first.

5. I consider myself as an Asian-American. I have both Asian and American characteristics, and I view myself as a blend of both. 
SL-ASIA, page 1

\title{
SUINN-LEW ASIAN SELF-IDENTITY ACCULTURATION SCALE \\ SUINN-LEW Tự Lựong Định Văn Hóa Ngươi Á Đông
}

\author{
(SL-ASIA)
}

\section{Vletnamese \\ Ngươi Việtnam}

\section{INSTRUCTIONS: I am interested in finding out about your cultural}

Phần Hướng Dẫn: Tôi rất thích thú trong việc tìm hiểu văn hóa của bạn. Có 26 câu hỏi

identity. There are 26 questions about your historical background and more về qúa trình hiểu biết và cách xử thế gần đây của bạn có liên quan đến sự lượng dịnh

recent behaviors which may be related to your cultural Identity. I will read the văn hóa cúa bạn. sẽ thông dịch câu hỏi của tôi với 5 câu trá lời cho

question. will interpret the question. Then I will give you flve mồi câu hỏi. Bạn vui lòng cho tôi biết câu trá lời nào mà bạn nghỉ đúng nhất cưa bạn.

possible answers. Tell me which one answer best describes you. If you forget the Nểu bạn quên những câu trả lời, Chúng tôi sẽ lập lại cho bạn lần nữa.

choices, we will give them to you again.

1. What language can you speak?

Ngôn ngũ bạn thường dùng là gì.

1. Vietnamese only

Chỉ tiếng Việt mà thôi

2. Mostly Vietnamese, some English

Phà̀n lớn là tiếng Việt

3. Vietnamese and English about equally well (bilingual)

Cả tiểng Việt và tiếng Anh

4. Mostly English, some Vietnamese

Phần lớn là tiếng Anh và chủ ít tiếng Việt

5. Only English

Chỉ tiếng Anh mà thôi 
SL-ASIA, page 2

2. What language do you prefer?

Bạn thích xử dụng loại ngôn ngũ nào?

1. Vietnamese only

Chi tiếng Việt mà thôi

2. Mostly Vietnamese, some English

Phần lớn là tiếng Việt

3. Vietnamese and English about equally well (bllingual)

Cả tiếng Việt và tiếng Anh

4. Mostly English, some Vietnamese

Phần lớn là tiếng Anh và chỉ ít tiếng Việt

5. Only English

Chì tiếng Anh mà thôi

3. How do you identify yourself?

Bạn tự nhận mình là người thế nào?

1. Oriental

Ơ Đông Phương

2. Asian

Á Đông

3. Astan-American

Á Đông và $M \bar{y}$

4. Vietnamese-American

Cả Việt và $M \bar{y}$

5. American

Người $M \bar{y}$ 
SL-ASIA. page 3

4. Which identification does (did) your mother use?

Mẹ của bạn tự nhận mình là người thế nào?

1. Oriental

ơ Đông Phương

2. Asian

Á Đông

3. Asian-American

Á Đông và $M \bar{y}$

4. Vletnamese-American

Cả Việt và $M \bar{y}$

5. American

Người $M \bar{y}$

5. Which identification does (did) your father use?

Cha của bạn tự nhận mình là người thế nào?

1. Oriental

Ơ Đông Phương

2. Asian

Á Đông

3. Asian-American

Á Đông và $M \bar{y}$

4. Vletnamese-American

Cả Việt và $M \bar{y}$

5. American

Người Mỹ 
6. What was the ethnic origin of the friends and peers you had, as a child Nhưng người bạn của bạn, từ khi còn thơ ấu cho đến lúc 6 tuối, họ thuộc dân tộc nào? up to age $6 ?$

1. Almost exclustvely Asians, Astan-Americans, Orientals Chỉ riêng là người Á Đông

2. Mostly Aslans, Asian-Americans, Orientals Phần đông là người Á Đông

3. About equally Asian groups, and Anglo groups Cả người $A$ Đông lẩn người $M y \overline{~ d o ̂ ̀ n g ~ đ e ̂ ̀ u ~}$

4. Mostly Anglos, Blacks, Hispanics, or other non-Asian ethnic Phần đông là người Mỹ Trẳng, Mỹ Đen, Người Mề, và những dân tộc khác

groups

không thuộc Á Đông

5 Almost exclusively Anglos, Blacks, Hispanics or other non-Asian Chỉ riêng là người $M y \bar{y} T$ rắng, $M y \bar{y}$ Đen, Người Mề, và những dân tộc khác ethnic groups không thuộc Ả Đông 
SL-ASIA, page 5

7. What was the ethnic origin of the friends and peers you had, as a child Những người bạn của bạn từ 6 tuởi đến 18 tuối, họ thuộc dân tộc nào?

from 6 to 18 ?

1. Almost exclustvely Asians, Astan-Americans, Orientals Chỉ riêng là người Á Đông

2. Mostly Astans, Asian-Americans, Orientals

Phân đông là người Á Đông

3. About equally Asian groups, and Anglo groups

Cả người Á Đông lẩn người $M y \bar{y}$ đồng dều

4. Mostly Anglos, Blacks, Hispanics, or other non-Asian ethnic

Phần đông là người $M \bar{y}$ Trắng, $M \bar{y}$ Đen, Người Mễ, và những dân tộc khác

groups

không thuộc Á Đông.

5 Almost exclustvely Anglos, Blacks, Hispanics or other non-Asian

Chí riêng là người $M \bar{y}$ Trắng, Mỹ Đen, Người Mễ, và những dân tộc khác

ethnic groups

không thuộc Á Đông 
SL-ASIA, page 6

8. Whom do you now associate with in the communitv?

Bạn thường giao tiếp với những ai trong cộng dồng?

1. Almost exclustvely Asians, Asian-Americans, Orientals

Chỉ riêng là người Á Đông

2. Mostly Asians, Asian-Americans, Orientals

Phần đông là người $A$ Đông

3. About equally Astan groups, and Anglo groups

Cả người Á Đông lẩn người $\mathrm{Mỹ}$ đồng đều

4. Mostly Anglos, Blacks, Hispanics, or other non-Astan ethnic

Phần đông là người $M \bar{y}$ Trắng, $M \bar{y}$ Đen, Người Mể, và nhūng dân tộc khác

$$
\text { groups }
$$

không thuộc Á Đông

5 Almost exclustvely Anglos, Blacks, Hispanics or other non-Asian

Chi riêng là người $M y ̃$ Trắng, Mỹ Đen, Người Mể, và những dân tộc khác

ethnic groups

không thuộc Á Đông. 
SL-ASIA, page 7

9. If your could pick. whom would you prefer to associate with in the Nều bạn có thế chọn, thì bạn thich kết thân với những ai trong cộng đồng ? community?

1. Almost exclusively Aslans, Aslan-Americans, Orientals Chi riêng là người Á Đông

2. Mostly Asians, Asian-Americans, Orientals Phần đông là người $A ́$ Đông

3. About equally Asian groups, and Anglo groups Cá người $A$ Đông lần người $M y ̃$ đông đều

4. Mostly Anglos, Blacks, Hispanics, or other non-Asian ethnic Phần đông là người $M \bar{y}$ Trắng, Mỹ Đen, Người Mề, và những dân tộc khác groups không thuộc Á Đông.

5 Almost exclusively Anglos, Blacks, Hispanics or other non-Asian Chỉ riêng là người $M \bar{y}$ Trắng, $M y \bar{y}$ Đen, Người Mề, và những dân tộc khác ethnic groups

không thuộc Á Đông. 
SL-ASIA, page 8

10. What is your music preference?

Bạn thường thích nghe loại nhạc gì?

1. Only Asian music (for example, Chinese, Vietnamese)

Chí nhạc Á Đông thôi (thí dụ nhạc Trung Hoa hoặc nhạc Việt Nam)

2. Mostly Asian

Phần lớn nhạc Á Đông

3. Equally Asian and English

Cả nhạc $\mathrm{A}$ Dông và nhạc $\mathrm{M} \bar{y}$ bằng nhau

4. Mostly English

Phần lớn là nhạc $M \bar{y}$

5. English only

Chi nhạc Mỹ thôi

11. What is your movie preference?

Bạn thích xem những loại phim nào?

1. Asian-language movies only

Chi phim Á Đông thôi (thí dụ phim Trung Hoa hoặc phim Việt Nam)

2. Astan-language movies mostly

Phần nhiều là phim Á Đông

3. Equally Asian/English

Cả phim Mȳ lần phim Việt bằng nhau

4. English-language movies mostly

Phần nhiều là phim $\mathrm{My}$

5. Englush-language movies only

Chi phim tiềng Anh thôi 
SL-ASIA, page 9

12. Where were you born?

Bạn sanh tại dâu?

U.S. US. Vietnam $\quad$ Other - Where

Where was your father born?

Cha của bạn sanh tại đâu?

UU.S. Vietnam $\quad$ Other - Where

Where was your mother born?

Mẹ của bạn sanh tại đâu?

ZU.S. US. Vietnam $\quad$ Other - Where

Where was your father's father born?

Ông Nội của bạn sanh tại đâu?

_U.S. Vietnam US. Other - Where

Where was your father's mother born?

Ông Ngoại của bạn sanh tại đâu?

U.S.

Vietnam

Other - Where

US.

Vietnam Nơi nào khác - tại đâu

Where was your mother's father born?

Bà Nội của bạn sanh tại đâu?

U.S. Vietnam Other - Where US. Vietnam Nơi nào khác - tại đâu

Where was your mother's mother born? Bà Ngoại của bạn sanh tại đâu?

U.S. Vietnam Other - Where US. Vietnam Nơi nào khác - tại đâu 
On the basis of the above answers, which generation best applies to you:

Căn cứ vào nhửng câu trá lời trên, thì bạn thuộc về thế hẹ nào ?

1. 1st Generation = I was born in Asia or other

Thế hệ thử I. Tôi sinh ở Á Đông hoặc nơi nào khác

2. 2nd Generation = I was born in U.S., either parent was born in

Thế hệ thư II. Tôi sanh ở Mỹ, nhưng một trong hai cha mẹ tôi sanh ở Á Đông

Asla or other

3. 3rd Generation = I was born in U.S., both parents were born in

Thế hệ thứ III. Tôi sanh ơ $M \bar{y}$, cả cha mẹ tôi cùng sanh ở $M \bar{y}$. Còn tất cả nội

U.S.. and all grandparents born in Asla or other

ngoại tôi đều sanh ở Á Đông hoặc noi nào khác

4. 4th Generation = I was born in U.S., both parents born in U.S..

Thể hệ thư IV. Tôi sanh ơ $M \bar{y}$, cha mẹ tôi sanh ở $M \bar{y}$, và it nhất là một trong bôn

and at least one grandparent born in Asla or other

ông bà nội ngoại tôi cūng sanh ở $\mathrm{My}$.

and one grandparent born in U.S.

5. 5th Generation $=I$ was born in U.S., both parents and all

Thế hệ thư $V$. Tôi sanh ở $M \bar{y}$, cha mẹ tôi sanh ở $M \bar{y}$, và tất cả ông bà nội ngoại

grandparents also born in U. S.

tôi dều sanh ở Mȳ.

6. Don't know what generation best fits since I lack some

Tôi không biết tôi thuộc thế hệ nào, vì tôi không có dủ các dư kiện về việc đó.

information. 
SL-ASIA, page 11

13. Where were you raised?

Bạn được nuôi đưỡng ở đâu?

1. In Asta only Chì tại Á Đông thôi

2. Mostly in Asia, some in U.S.

Phần lớn thi ở ÁĐông

3. Equally in Asta and U.S.

Cả tại Á Châu lẩn Hoa Kỳ đồng đều

4. Mostly in U.S., some in Asia

Phân lớn là tại $M \bar{y}$, thời gian ngắn tại Á Châu.

5. In U.S. only

Chi tại Hoa Kỳ thôi

14. What contact have vou had with Asia?

Bạn đã có những sự liên hệ thế nào tại Á Đông?

1. Raised one year or more in Asla

Được nuôi dưỡng 1 năm hoặc nhiều hơn nữa tại Á Đông

2. Lived for less than one year in Asia

Ơ Á Đông it hơn 1 năm

3. Occasional visits to Asia

Thinh thoảng thì có đi thăm Á Đông

4. Occasional communications (letters, phone calls, etc.) with people Thinh thoảng thì có gọi điện thoại hoặc viết thư cho người tại Ả Đông

in Asia

5. No exposure or communications with people in Asia

Không có liên hệ với người tại Á Đông 
SL-ASIA, page 12

15. What is your food preference at home?

Bạn thich ān loại thực phẩm nào ở nhà bạn?

1. Exclusively Asian food

Chi ăn đồ ăn Á Đông thôi

2. Mostly Astan food, some American

Phần lớn là ăn đồ ăn Á Đông và cūng ăn một ít đồ ăn $M \bar{y}$

3. About equally Asian and American

Thức ăn của $M \bar{y}$ và thức ăn của Á Đông bằng nhau

4. Mostly American food

Phần lớn là đồ ăn $\mathrm{My}$

5. Exclusively American food

Chủ ăn đồ ăn $M \bar{y}$ thôi

16. What is your food preference in restaurants?

Bạn thích ăn đồ ăn gi khi đi ăn tại nhà hàng:

1. Exclustvely Asian food

Chỉ ăn đồ ăn Á Đông thôi

2. Mostly Asian food, some American

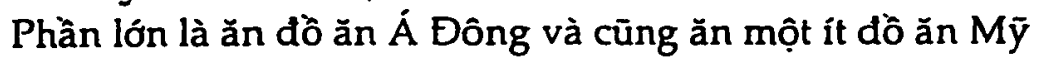

3. About equally Asian and American

Thức ăn cưa $M \bar{y}$ và thức ăn của $A$ Đông bằng nhau

4. Mostly American food

Phần lớn là đồ ăn $\mathrm{My}$

5. Exclusively American food

Chỉ ăn đồ ăn Mỹ thôi 
SL-ASIA, page 13

17. Do you

Có phải Bạn ưa thích:

1. read only an Asian language

Chi dọc ngôn ngũ Á Đông mà thôi

2. read an Asian language better than English Đọc tiếng Á Đông hơn, vì rành hơn tiếng Anh

3. read both Asian and English equally well Đọc tiếng Anh và cá tiếng Việt ngang nhau

4. read English better than an Asian language Đọc tiếng $A n h$, vì rành tiếng Anh hơn tiếng Việt

5. read only English

Chi đọc tiếng Anh mà thôi

18. Do you

Bạn có khả năng:

1. write only an Asian language Chì dọc ngôn ngữ Á Đông mà thôi

2. write an Asian language better than English Đọc tiếng Á Đông hơn, vì rành hơn tiếng Arh

3. write both Aslan and English equally well Đọc tiếng Anh và cả tiếng Việt ngang nhau

4. write English better than an Asian language Đọc tiếng Anh, vì rành tiếng Anh hơn tiếng Việt

5. write only English

Chỉ dọc tiếng Anh mà thôi 
SL-ASIA, page 14

19. If you consider yourself a member of the Asian group (Oriental. Asian. Nếu bạn coi minh thuộc về cộng đồng Á Đông(khi ở đông phương, ở Á Đông, ơ Vietnamese, Vietnamese-American etc., whatever term you prefer), how Vietnam, ở cả Mỹ và Việt Nam v.v...) thì sự hānh diện của bạn đến mức độ nào? much pride do you have in this group?

1. Extremely proud

Vô cừng hānh diện

2. Moderately proud hānh diện vừa vữa

3. Little pride

Ít hānh diện

4. No pride but do not feel negative toward group

Không hānh diện mà cūng không mặc cảm gì với cộng đồng

5. No pride but do feel negative toward group

Không hānh diện nhưng còn cảm thấy mặc cám về cộng đồng

20. How would you rate yourself?

Bạn tự kế mình là người như thế nào?

1. Very Aslan

Rất Á Đông

2. Mostly Aslan

Phần lớn là ÁĐông

3. Bicultural

Thuộc về cá hai văn hóa

4. Mostly Westernized

Phần lớn đã Tây Phương hóa

5. Very Westernized

Rất là Tây Phương hóa 
SL-ASIA. page 15

21. Do you participate in Astan (Vietnamese) occasions, holidays, traditions.

Bạn có tham dự vào các dịp lể lạc hay là các truyền thống văn hoá Á Đông (Vietnam)

etc.?

không?

1. Nearly all

Gần nhu hết mọi dịp

2. Most of them

Phần nhiều dịp

3. Some of them

Một vài dịp

4. A few of them

Rất it dịp

5. None at all

Không tham dự vào dịp nào hết

22. Rate yourself on how much you belleve in Aslan values (e.g., about Tự lượng định về các gía trị Đông phương mà ban tin tường.

marriage, families, education, work):

1. 2 .

Do not belleve in Asian values

Không tin tường vào gia tri Đông Phương
3.

Strongly belleve in Asian values

Tin tướng rất mạn vào gia trị Đông Phương

23. Rate yourself on how much you believe in American (Westem) values: Tự lượng dịnh về các gia trị Tây Phương mà ban tin tường.?
1.
2.
3.
4.
5.

Do not belleve in American values

Strongly believe in Amertcan values

Không tin tướng vào gia trị Tây Phương

Tin tương rãt mạnh vào gía trị Tây Phương 
SL-ASIA, page 16

24. Rate yourself on how well you fit when with other Vietnamese Tự lượng định sự thích hợp của bạn những người Việtnam khac.

1.

Do not fit

Không thich hợp
2.

3.

4

4.

5.

Fit very well Răit thích hợp

25. Rate yourself on how well you fit when with other Americans who are Tự lượng dịnh sự thích hợp của bạn những người $M \bar{y}$.

non-Astan (Westerners):

1.

2.

Do not nt

Không thích hợp
3.

4.

5.

Fit very well

Rất thich họp

26. There are many different ways in which people think of themselves. Có rất nhiều cách khác nhau để con ngưòi tự nghĩ về mình. Điều nào sau đây mà Which ONE of the following most closely describes how you view bạn nhận răng trung thật về con người bạn nhất.

yourself? 
SL-ASIA, page 17

1. I consider myself basically an Asianperson (e.g. Vietnamese). Even Tôi tự nhận mình trên căn bản như là một người Việtnam ( hay Á Đông) mạc dù though I live and work in America. I still view myself basically as tôi sống va làm việc tại $M \bar{y}$.

an Asian person.

2. I consider myself basically as an American. Even though I have an Tôi tự nhận minh trên căn bản như là người $M \bar{y}$, mạc dù tôi có đầy đủ truyền Asian background and characteristics, I still vlew myself basically as thống và dặc điếm của người Việtnam( hay Á Đông).

an American.

3. I consider myself as an Astan-American, although deep down I Tôi tự nhận mình như là một người Việt - Mỹ, mạc dù trong tận cùng tôi luôn always know I am an Asian. luôn biết tôi là người Việtnam( hay Á Đông).

4. I consider myself as an Asian-American, although deep down, I Tôi tự nhận mình như là một người Việt - Mỹ, mạc dù trong tận cùng tôi luôn view myself as an American first. luôn nhìn nhận như là người $M \bar{y}$ trước tiên.

5. I consider myself as an Aslan-Amertcan. I have both Asian and Tôi tự nhận mình như là một người Việt - Mỹ. Tôi có đủ cả hai đặc tính cúa American characteristics. and I vlew myself as a blend of both. người Việt và người $M \bar{y}$ 


\section{Appendix F3}

ASIA-SL

p. 1

\section{SUINN-LEW ASIAN SELF-IDENTITY ACCULTURATION SCALE (SL-ASIA) \\ Hmong}

INSTRUCTIONS: I am interested in finding out about your cultural identity. There are 26

Kuv xav paub txog nej tib neeg hmoob kevcai. Muaj nees nkaum rau yam lus

questions about your historical background and more recent behaviors which may be related to your noog txog nej tsev neeg thiab teeb meem. los, npas ham hais txog nej cov tib neeg. Kuv yuav nyeem

cultural identity. I will read the question. Then I will give you five possible answers. Tell me which txog yam ab tsi. Kuv ma lis muab tsib yam lus rau nej teb. Qhia kuv saib cov lus tw hais txog lossis

one answer best describes you. If you forget the choices, I will give them to you again.

ntxim nej tsheeb. Yog nej tsi nco qab. kuv ma lis rov qab nyeem cov lus ntawd dua rau nej.

1. What language can you speak?

Koj paub yam lus ab tsi?

1. Hmong only

Lus Hmoob xwb

2. Mostly Hmong, some English

Lus Hmoob ntau, lus Askiv tsawg

3. Hmong and English about equally well (bilingual)

Lus Hmoob thiab lus Askiv sib npaug

4. Mostly English, some Hmong

Lus Askiv ntau, lus Hmoob tsawg

5. Only English

Lus Askiv xwb

2. What language do you prefer?

Koj xav paub/nyiam yam lus twg?

1. Hmong only

Lus Hmoob xwb

2. Mostly Hmong, some English

Lus Hmoob ntau, lus Askiv tsawg

3. Hmong and English about equally well (bilingual)

Lus Hmoob thiab lus Askiv sib npaug

4. Mostly English, some Hmong

Lus Askiv ntau, lus Hmoob tsawg

5. Only English

Lus Askiv xwb 
ASIA-SL

p. 2

3. How do you identify yourself?

Koj yog neeg ab tsi?

1. Oriental

Suav/Yij Pooj

2. Asian

Ixias

3. Asian-American

Ixias-Asmeslivkas

4. Hmong-American

Hmoob-Asmeslivkas

5. American

Asmeslivkas

4. Which identification does (did) your mother use?

Koj niam yog neeg ab tsi?

1. Oriental

Suav/Yij Pooj

2. Asian

Ixias

3. Asian-American

Ixias-Asmeslivkas

4. Hmong-American

Hmoob-Asmeslivkas

5. American

Asmeslivkas 
ASIA-SL

p. 3

5. Which identification does (did) your father use?

Koj txiv yog neeg ab tsi?

Oriental
Suav/Yij Pooj

2. Asian

Ixias

3. Asian-American

Ixias-Asmeslivkas

4. Hmong-American

Hmoob-Asmeslivkas

5. American

Asmeslivkas

6. What was the ethnic origin of the friends and peers you had, as a child up to age 6 ?

Thaum koj me me quav, txij li 6 xyoos tej ntawd, koj cov phoojywg yog neeg ab tsi?

1. Almost exclusively Asians, Asian-Americans, Orientals

Neeg Ixias, Ixias-Asmeslivkas, Suav/Yij Pooj.

2. Mostly Asians, Asian-Americans, Orientals

Neeg Ixias, Ixias-Asmeslivkas, Suav/Yij Pooj.

3. About equally Asian groups, and Anglo groups

Neeg Ixias thiab neeg Asmeslivkas sib npaug.

4. Mostly Anglos, Blacks. Hispanics, or other non-Asian ethnic groups

Neeg Asmeslivkas, Asmeslivkas Dub, Mev, thiab yam neeg tsis yog neeg Ixias ntau.

$5 \quad$ Almost exclusively Anglos, Blacks, Hispanics or other non-Asian ethnic groups Neeg Asmeslivkas, Asmeslivkas Dub, Mev, thiab yam neeg tsis yog neeg Ixias. 
ASIA-SL

p. 4

7. What was the ethnic origin of the friends and peers you had, as a child from 6 to 18 ?

Thaum koj muaj li rau xyoos txog kaum yim xyoos, koj cov phoojywg yog neeg ab tsi?

1. Almost exclusively Asians, Asian-Americans, Orientals Neeg Ixias, Ixias-Asmeslivkas, Suav/Yij Pooj.

2. Mostly Asians, Asian-Americans, Orientals Neeg Ixias, Ixias-Asmeslivkas, Suav/Yij Pooj.

3. About equally Asian groups, and Anglo groups Neeg Ixias thiab neeg Asmeslivkas sib npaug.

4. Mostly Anglos, Blacks, Hispanics, or other non-Asian ethnic groups Neeg Asmeslivkas, Asmeslivkas Dub, Mev, thiab yam neeg tsis yog neeg Ixias ntau.

5 Almost exclusively Anglos, Blacks, Hispanics or other non-Asian ethnic groups Neeg Asmeslivkas, Asmeslivkas Dub, Mev, thiab yam neeg tsis yog neeg Ixias.

8. Whom do you now associate with in the community?

Lub caij no, koj nrov tib neeg ab tsi ua ke?

I. Almost exclusively Asians, Asian-Americans, Orientals

Neeg Ixias, Ixias-Asmeslivkas, Suav/Yij Pooj.

2. Mostly Asians, Asian-Americans, Orientals

Neeg Ixias, Ixias-Asmeslivkas, Suav/Yij Pooj.

3. About equally Asian groups, and Anglo groups Neeg Ixias thiab neeg Asmeslivkas sib npaug.

4. Mostly Anglos, Blacks, Hispanics, or other non-Asian ethnic groups Neeg Asmeslivkas. Asmeslivkas Dub, Mev, thiab yam neeg tsis yog neeg Ixias ntau.

5 Almost exclusively Anglos, Blacks, Hispanics or other non-Asian ethnic groups Neeg Asmeslivkas, Asmeslivkas Dub, Mev, thiab yam neeg tsis yog neeg Ixias. 
p. 5

9. If you could pick, whom would you prefer to associate with in the community?

Yog koj xaiv tau raws koj siab nyiam, koj xav nrog tib neeg twg ua ke?

1. Almost exclusively Asians, Asian-Americans, Orientals Neeg Ixias, Ixias-Asmeslivkas, Suav/Yij Pooj.

2. Mostly Asians, Asian-Americans, Orientals Neeg Ixias, Ixias-Asmeslivkas, Suav/Yij Pooj.

3. About equally Asian groups, and Anglo groups Neeg Ixias thiab neeg Asmeslivkas sib npaug.

4. Mostly Anglos, Blacks, Hispanics, or other non-Asian ethnic groups Neeg Asmeslivkas, Asmeslivkas Dub, Mev, thiab yam neeg tsis yog neeg Ixias ntau.

5 Almost exclusively Anglos, Blacks, Hispanics or other non-Asian ethnic groups Neeg Asmeslivkas, Asmeslivkas Dub, Mev, thiab yam neeg tsis yog neeg Ixias.

10. What is your music preference?

Koj nyiam mloog peej ab tsi?

1. Only Asian music (for exampie, Chinese, Hmong) Ixias li xwb

2. Mostly Asian

Ixias li ntau

3. Equally Asian and English

Ixias thiab Askiv sib npaug

4. Mostly English

Askiv li ntau

5. English only

Askiv xwb 
ASIA-SL

p. 6

11. What is your movie preference?

Koj nyiam xav saib na ab tsi?

1. Asian-language movies only

Ixias lus $x w b$

2. Asian-language movies mostly

Ixias lus ntau

3. Equally Asian/English

Ixias thiab Askiv sib npaug

4. English-language movies mostly

Askiv lus ntau

5. English-language movies only

Askiv lus xwb

12. Where were you born?

Koj yug lub tebchaws twg tuaj?
U.S.
Laos
Asmeslivkas Los Tsuas
Other - Where
Tebchaws twg

Where was your father bom?

Koj txiv yug tebchaws twg tuaj?
U.S.
Asmeslivkas Los Tsuas
Other - Where
Tebchaws twg

Where was your mother borm?

Koj niam yug tebchaws twg tuaj?

$\begin{array}{ll}\text { U.S. } & \text { Laos } \\ \text { Asmeslivkas Los Tsuas - Where } & \text { Tebchaws twg }\end{array}$

Where was your father's father born?

Koj yawg yug tebchaws twg?

$\begin{array}{ll}\text { U.S. } & \text { Laos } \\ \text { Asmeslivkas Los Tsuas - Where } & \text { Tebchaws twg }\end{array}$

Where was your father's mother born?

Koj pog yug tebchaws twg?

U.S. Laos Other - Where

Asmeslivkas Los Tsuas Tebchaws twg

Where was your mother's father born?

Koj yawm txiv yug tebchaw twg?

$\begin{array}{ll}\text { U.S. } & \text { Other - Where } \\ \text { Asmeslivkas Los } & \text { Los Tsuas }\end{array}$


ASIA-SL

p. 7

Where was your mother's mother bom?

Koj niam tais yug tebchaws twg tuaj?

U.S. Laos Other - Where

Asmeslivkas Los Tsuas Tebchaws twg

On the basis of the above answers, which generation best applies to you:

1. Ist Generation $=\mathrm{I}$ was born in Asia or other

Tiam $\mathrm{ib}=$ kuv yug tebchaw

2. 2nd Generation = I was born in U.S., either parent was born in Asia or other Tiam ob = Kuv yug tebchaws Asmeslivkas, kuv niam kuvtxiv yug tebchaws

3. 3rd Generation = I was born in U.S., both parents were born in U.S., and all Tiam peb = kuv yug tebchaws Asmeslivkas, kuv niam kuv txiv nkawd yug hauv

grandparents born in Asia or other tebchaws Asmeslivkas, niam tais yawm txiv thiab pog yawg lawv sawsdaws yug tebchaws Asmeslivkas.

4. 4th Generation = I was born in U. S., both parents bom in U.S., and at least one Tiam plaub = Tas nrog kuv tsev neeg yug tebchaws Asmeslivkas tshuav kuv niam

grandparentborn in Asia or other and one grandparent born in U.S. pog txiv yawg los yawm txiv thiab niam tais tiaj li yug tebchaws xwb.

5. Sth Generation = I was born in US, both parents and all grandparents also born in US.

Tiam tsib = Peb puavleej tuaj yug Asmeslivkas tebchaws.

6. Don't know what generation best fits since I lack some information.

Kuv tsis paub.

13. Where were you raised?

Koj tuaj hlob tebchaws tw?

1. In Asia only

Ixias xwb

2. Mostly in Asia, some in U.S.

Hlob tebchaw Ixias ntau ma li tuag tebchaw Asmeslivkas.

3. Equally in Asia and U.S.

Hlob tebchaws Ixias thiab Asmeslivkas sib npaug

4. Mostly in U.S., some in Asia

Hob tebchaw Asmeslivkas

5. In U.S. only

Hlob hauv Ásmeslivkas xwb 
ASIA-SL

p. 8

14. What contact have you had with Asia?

Koj pua hnov moos txog tib neeg nyob Ixias?

1. Raised one year or more in Asia

Nyob ib xyoos tim tebchaw Ixias xwb

2. Lived for less than one year in Asia

Nyob tsis ntev ib xyoos tim tebchaw Ixias

3. Occasional visits to Asia

Muaj sijhawm mus saib tebchaws Ixias

4. Occasional communications (letters, phone calls, etc.) with people in Asia Muaj sijhawm sau ntawv thiab hu xov tooj nstib kwvtij neejtsa txheeb ze

5. No exposure or communications with people in Asia

Tsis pom thiab tsis hnov moos txog tib neeg nyob tebchaw Ixias li

15. What is your food preference at home?

Koj nyiam noj hom mov ab tsi?

1. Exclusively Asian food

Mov Ixias xwb

2. Mostly Asian food, some American

Ixias suab mov ntau. Asmeslivkas li tsawg

3. About equally Asian and American

Ixias thiab Asmeslivkas li sib npaug

4. Mostly American food

Asmeslivkas li ntau

5. Exclusively American food

Asmeslivkas li mov xwb 
ASIA-SL

p. 9

16. What is your food preference in restaurants?

Yog koj noj mov hauv tablaj, koj nyiam noj ham mov ab tsi?

1. Exclusively Asian food

Ixias li mov $x w b$

2. Mostly Asian food, some American

Ixias li mov ntau, Asmeslivkas li tsawg

3. About equally Asian and American

Ixias thiab Asmeslivkas li mov sib npaug

4. Mostly American food

Asmeslivkas li mov ntau

5. Exclusively American food

Asmeslivkas saub mov $\mathrm{xwb}$

17. Do you

Koj

1. read only an Asian language pub nyeem ntawv Ixias $x w b$

2. read an Asian language better than English nyeem ntawv Ixias zoo tshaj ntawv Askiv

3. read both Asian and English equally well paub nyeem ntawv Ixias thiab ntawv Askiv tibsi

4. read English better than an Asian language nyeem ntawv Askiv zoo tshaj ntawv Ixias

5. read only English

paub nyeem ntawv Askiv xwb 
ASIA-SL

p. 10

18. Do you

Koj

I. write only an Asian language

sau ntawv Ixias Xwb

2. write an Asian language better than English

sau ntawv Ixias zoo tshaj ntawv Askiv

3. write both Asian and English equally well paub sau ntawv Ixias thiab Askiv tibsi

4. write English better than an Asian language sau lus Askiv zoo tshaj lus Ixias

5. write only English

paub sau lus Askis xwb

19. If you consider yourself a member of the Asian group (Oriental, Asian, Hmong, HmongTsuav koj yog yam neeg ab tsi (Suav/Yij Pooj, Ixias, Hmoob, Hmoob-Asmeslivkas) koj

American etc., whatever term you prefer), how much pride do you have in this group? pua zoo siab tia koj yog yam neeg ntawd?

1. Extremely proud

zoo siab heev

2. Moderately proud

zoo siab

3. Little pride

zoo siab me me

4. No pride but do not feel negative toward group

Txajmuag, tab sis tsis xav li cas

5. No pride but do feel negative toward group

Txajmuag heev, xav tau teeb meem phem 
ASIA-SL

p. II

20. How would you rate yourself?

Hauv koj lub siab, koj pua xav tias koj?

1. Very Asian

Yeej yog neeg Ixias $x w b$.

2. Mostly Asian

Yog neeg Ixias ntau

3. Bicultural

Ixias thiab Asmeslivkas tib si

4. Mostly Westemized

Zoo li neeg Askiv lawm

5. Very Westemized

Yeej yog neeg Askiv lawm

21. Do you participate in Asian (Hmong) occasions, holidays, traditions, etc.?

Txog caij Hmoob ua lom zem loj, koj puas nrog lawm sib koom ua?

1. Nearly all

Ua tas nhro

2. Most of them

Ua raws li siab nyiam

3. Some of them

Ua tej qhov xwb

4. A few of them

Ua me me

5. None at all

Tsis ua li

22. Rate yourself on how much you believe in Asian values (e.g., about marriage, families, Koj ntseeg Hmoob kevcai npaum li cas? (kev sib yuav, niamtxiv kwvtij neejtsa thiab kev

education, work):

kaum ntawv thiab kev ua haujlwm):

$1 . \quad 2$.

Do not believe in Asian value Ntseeg me me xwb
3.

4

Strongly believe in Asian values

Ntseeg loj 
ASIA-SL

p. 12

23. Rate yourself on how much you believe in America (Western) values:

Koj ntseeg Asmeslivkas kevcai npaum li cas?

1. 2.23.

Do not believe in American values

Ntseeg me me xwb
3.

4.

Strongly believe in American values Ntseeg loj

24. Rate yourself on how well you fit when with other Hmong people.

Koj pua paub nrog Iwm yam Hmoob tham pem ua kev?
1.
2.
3.
4.
5.

Do not fit

Tsis puab li

Fit very well paub

25. Rate yourself on how well you fit when with other Americans who are non-Asian (Westerners):

Koj pua paub nrog lwm yam neeg Askiv, tsi yog neeg Ixias, sib xyaws?
1.
2.
4.
Fit very well
Tsis paub paub

26. There are many different ways in which people think of themselves. Which ONE of the Muaj ntau yam kev tib neeg nyia xav txog nyia tus kheej. Koj xav sem cov lus hauv puas

following most closely describes how you view yourself? yog hais raws li koj siab xav txog koj tus kheej? 
ASLA-SL

p. 13

1. I consider myself basically an Asianperson (e.g. Hmong). Even though I live and Kuv nyob thiab ua haujlwm hauv tebchaws Asmeslivkas, tab sis kuv yeej yog neeg

work in America, I still view myself basically as an Asian person.

Ixias hauv kuv lub siab.

2. I consider myself basically as an American. Even though I have an Asian backgroundand characteristics, I still view myself basically as an American.

Kuv ntseeg Ixias kevcai, tab sis mas kuv xav tias kuv yeej yog tib neeg Asmeslivkas.

3. I consider myself as an Asian-American, although deep down I always know I am Kuv xav tias kuv yog Hmoob-Asmeslivkas, tab sis hauv kuv lub siab, kuv yog

an Asian.

neeg Ixias.

4. I consider myself as an Asian-American, although deep down, I view Kuv xav tias kuv yog Ixias-Asmeslivka, tab sis hauv kuv siab, kuv paub tias kuv myself as an American first. yog neeg Asmeslivkas ua ntej.

5. I consider myself as an Asian-American. I have both Asian and American Kuv yog neeg Ixias-Asmeslivkas. Kuv paub kevcai Ixias thiab kevcai Asmeslivkas characteristics, and I view myself as a blend of both. sib npaug. 


\section{Appendix G}

\section{Hopkins Symptom Checklist-25}

1. Vietnamese Version . . . . . . . . . . . . . . 202

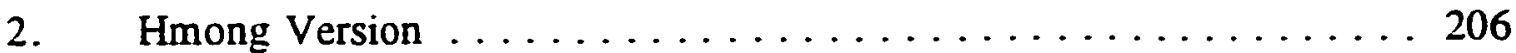




\section{HOPKINS SYMPTOM CHECKLIST 25}

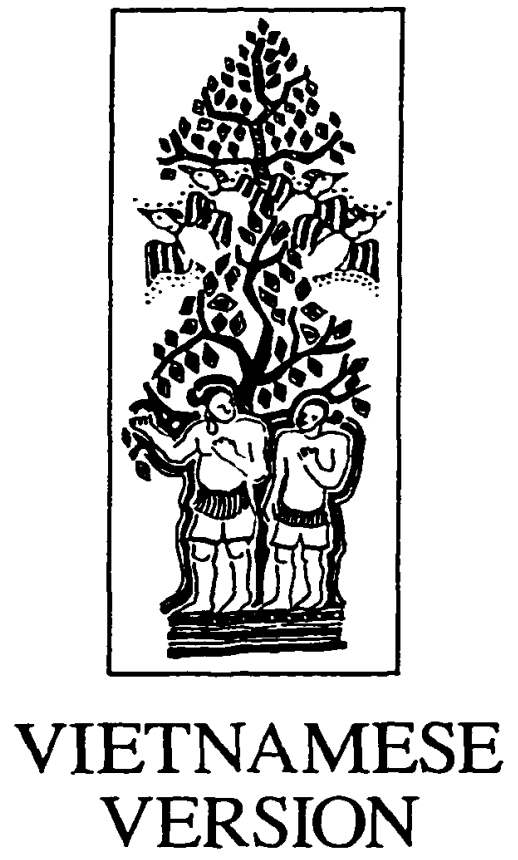

NAME IATE CLINICIAN

DATE OF BIRTH SF:X MARITAL STATUS 


\section{SCORING}

Responses are summed and divided by the number of answered items, to generate three scores:

$$
\begin{aligned}
\text { ANXIETY } & =\frac{\text { ITEMS } 1-10}{10} \rightarrow \\
\text { DEPRESSION } & =\frac{\text { ITEMS } 11-25}{15} \rightarrow \square \\
\text { TOTAL } & =\frac{\text { ITEMS } 1-25}{25} \rightarrow \square
\end{aligned}
$$

Individuals with scores on anxiety and/or depression and/or total $>1.75$ are considered symptomatic.

See Manual for additional information. 


\section{Instructions}

Listed below are some symptoms or problems that people sometimes have. Please read each one carefully and decide how much the symptoms bothered or distressed you in the last week. including today. Place a check in the appropriate column.

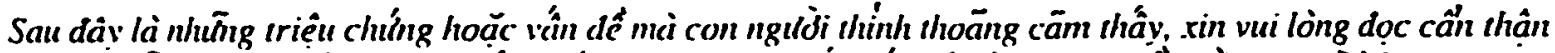

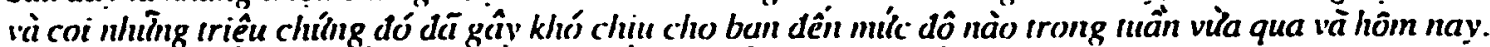

Xin ghi vào nhïing cột thích úng.

\begin{tabular}{|c|c|c|c|c|c|}
\hline & & 1 & 2 & 3 & 4 \\
\hline \multirow{2}{*}{\multicolumn{2}{|c|}{$\begin{array}{l}\text { PART I } \\
\text { ANXIETY SYMPTOMS }\end{array}$}} & Not at all & A little & Quitc a bit & Extremely \\
\hline & & Khîng hê" an & Crichún ai & Bi khá nhû̀u & B! rist ninsti \\
\hline $\mathrm{I}$. & $\begin{array}{l}\text { Suddenly scared for no reason } \\
\text { Bổng dthing lo sư mà không lí des }\end{array}$ & & & & \\
\hline 2. & $\begin{array}{l}\text { Feeling fearful } \\
\text { Câin giác sơ hadi }\end{array}$ & & & & \\
\hline 3. & $\begin{array}{l}\text { Faintness, dizziness, or weakness } \\
\text { Gần ngất xỉu, ngất xìu, chóng mặt, suy yếu }\end{array}$ & & & & \\
\hline 4. & $\begin{array}{l}\text { Nervousness or shakiness inside } \\
\text { Run sợ hoăc hồi họp trong lòng }\end{array}$ & & & & \\
\hline 5. & $\begin{array}{l}\text { Heart pounding or racing } \\
\text { Tim dập manh, hoặc nhanh }\end{array}$ & & & & \\
\hline 6. & $\begin{array}{l}\text { Trembling } \\
\text { Tự dung các báp thit bi giât, run (run rầy) }\end{array}$ & & & & \\
\hline 7. & $\begin{array}{l}\text { Feeling tense or keyed up } \\
\text { Câm giác căng thằng }\end{array}$ & & & & \\
\hline 8. & $\begin{array}{l}\text { Headaches } \\
\text { Bi nhúćc đầu }\end{array}$ & & & & \\
\hline 9. & $\begin{array}{l}\text { Spells of terror or panic } \\
\text { Bị nhiơng cơn hoáng hốt, lo sợ ngắn xấy ra }\end{array}$ & & & & \\
\hline 10. & $\begin{array}{l}\text { Feeling restless, can't sit still } \\
\text { Câm giác bồn chồn, lo lâng khó thể ngồi yên môt ndi }\end{array}$ & & & & \\
\hline
\end{tabular}




\begin{tabular}{|c|c|c|c|c|c|}
\hline \multicolumn{2}{|r|}{ 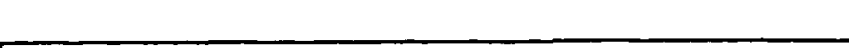 } & \multicolumn{2}{|c|}{$1 \quad 2$} & 3 & \multirow{2}{*}{$\frac{4}{\text { Extremely }}$} \\
\hline & PART II & Not at all & A litrle & Quite a hit & \\
\hline & DEPRESSION SYMPTOMS & Không hề \&ó & Cóchuír uí & Bịkhá nhu2ì & Bị râi nhiềi \\
\hline 11. & $\begin{array}{l}\text { Feeling low in energy, skowed down } \\
\text { Cám giác yću ớt, tế oái }\end{array}$ & & & & \\
\hline 12. & $\begin{array}{l}\text { Blaming yourself for things } \\
\text { Tîtrcich minh về moi viếc xây ra }\end{array}$ & & & & \\
\hline 13. & $\begin{array}{l}\text { Crying easily } \\
\text { Bậr khóc dế dàng }\end{array}$ & & & & \\
\hline 14. & $\begin{array}{l}\text { Luss of sexual interest or pleasure } \\
\text { Mất hay î hứng thui trong viêc sinht lý }\end{array}$ & & & & \\
\hline 15. & $\begin{array}{l}\text { Poor appetite } \\
\text { Äl không biếr ngon }\end{array}$ & & & & \\
\hline 16. & $\begin{array}{l}\text { Difficulty falling asleep. staying asleep } \\
\text { Khó dồ giấc ngü, khó yền gî́c ngú }\end{array}$ & & & & \\
\hline 17. & $\begin{array}{l}\text { Feeling hopeless about the future } \\
\text { Câm giác tujệt long về tuidng lai }\end{array}$ & & & & \\
\hline 18. & $\begin{array}{l}\text { Feeling blue } \\
\text { Câm giác âm-u, buồn nån }\end{array}$ & & & & \\
\hline 19. & $\begin{array}{l}\text { Feeling lonely } \\
\text { Câm giác cô dơn }\end{array}$ & & & & \\
\hline 20. & $\begin{array}{l}\text { Thoughts of ending your life } \\
\text { Cóý yư'̛̉ng muốn kết liếu cuộc ddi minh }\end{array}$ & & & & \\
\hline 21. & $\begin{array}{l}\text { Feeling of being trapped or caught } \\
\text { Cám giác mắc ket hoăc không lối thoát }\end{array}$ & & & & \\
\hline 22. & $\begin{array}{l}\text { Worrying too much about things } \\
\text { Lo lăng thái quá vếmoi viêc }\end{array}$ & & & & \\
\hline 23. & $\begin{array}{l}\text { Feeling no interest in things } \\
\text { Câm giác không ham thích gi núa, bất cứ việc gì }\end{array}$ & & & & \\
\hline 24. & $\begin{array}{l}\text { Feeling everything is an effort } \\
\text { Cäm giác. viêc gì cuñng khó khän dòi nhiêu cố gáng }\end{array}$ & & & & \\
\hline 25. & $\begin{array}{l}\text { Feelings of worthlessness } \\
\text { Cám giäc minh là kẻ bất tài, vô dung }\end{array}$ & & & & \\
\hline
\end{tabular}




\section{HOPKINS SYMPTOM CHECKLIST 25}

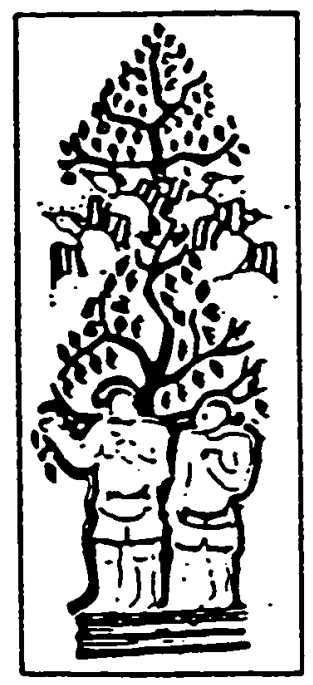

HMONG VERSION

NAME DATE CUNICIAN SEX makttal status

ARRISAL DATE FrCHIATRIC DIACNOSIS 


\section{SCORING}

Responses are summed and divided by the number of answered items. to generate three scores:

$$
\begin{aligned}
\text { ANXIETY } & =\frac{\text { ITEMS } 1-10}{10} \rightarrow \square \\
\text { DEPRESSION } & =\frac{\text { ITEMS } 11-25}{15} \rightarrow \square \\
\text { TOTAL } & =\frac{\text { ITEMIS } 1.25}{25} \rightarrow \square
\end{aligned}
$$

Individuals $u$ ith scores on anxiety and'or depression and/or lotal > 1.75 are considered symplomatic.

See Manual for additional information.

$$
\begin{gathered}
\text { Cccapled flum } \\
\text { deTEDY } \\
\text { INDOCHINESE PSYCHIATRY CLINIC } \\
\text { BRIGHTON MARINE PUBLIC HEALTH CENTER } \\
\text { DEPARTMENT OF PSYCHIATRY } \\
\text { ST. ELIZABETH'S HOSPITAL } \\
\text { AND }
\end{gathered}
$$

THE HARVARD PROGRAM IN PSYCHIATRIC EPIDEMIOLOGY

Funded by the U.S. Office of Refugee Resettlement O1986 Richard F. Mollica, M.D. 
LUS QHIA UA:

NRAM QAB NO YOG IB CO LUS UAS TIB NEEG NIAJ ZAUS MUAJ. THOV KOJ NYEEM

IB KAB LUS ZUJ ZUS ES XAV SAIB COV LUS NTAWD HAIS RAUG KOJ TUS KHEEJ NPAUM LI CAS NYOB RAU LUB LIM TIAM TAS LOS. CES KOJ MAM KHIJ RAU KEM UAS MUAJ NTAU, MUAJ ME NTSIS, MUAJ ME ME, LOS YOG TSIS MUAJ LI UAS NYOB RAU SAB XIX NTAWM DAIM NTAWV NO.

1. POOB SIAB YAM TSIS PAUB TIAS YOG TIM AB TSI Suddenly scared for no reason

2. MUAJ UUB SIAB PHEEJ NTSHAI............ Feeling fearful

3. TSAUS MUAG LOS YOG TSIS MUAJ ZOG......... Faintness, dizziness, or weakness

4. SIAB NYOB TSIS IUS LOS YOG LUB SIAB TSHEE. Nervousness or shakiness inside

5. LUB SIAB DHIA CEEV.

Heart pounding or racing

6. IB CE PHEES TSHEE.................. Trembling

7. NOOG 200 LI IB CE NRUJ NRUJ............. Feeling tense or keyed up

8. MOB TAUb haU. ....................... Headaches

9. POOB SIAB NTSHAI HEEV.................. Spells of terror or panic

10. NYOB tSIS taUS RAU IB QHO chaw LI........ Feeling restless, can't sit still

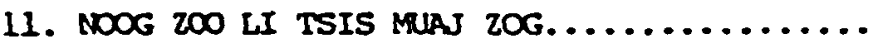
Feeling low in energy, slowed down

12. PHEEJ KIAM HAIS TIAS YOG TIM YUS......... Blaming yourself for things

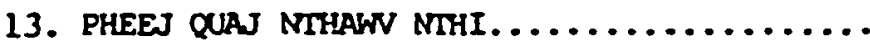
Crying easily

14. TSIS QAB SIAB NROG POJ NLAM (TXIV NEEJ) PU Loss of sexual interest or pleasure

\begin{tabular}{|c|c|c|c|}
\hline not at all & a litte & quite a bit & extrate \\
\hline $\begin{array}{l}\text { TSIS } \\
\text { MUAJ LI }\end{array}$ & $\begin{array}{l}\text { MUAJ } \\
\text { ME ME }\end{array}$ & $\begin{array}{c}\text { MUAJ } \\
\text { ME NTSIS }\end{array}$ & $\begin{array}{l}\text { MUAJ } \\
\text { NTAU HEEV }\end{array}$ \\
\hline & & & \\
\hline & & & \\
\hline & & & \\
\hline & & $\cdot$ & \\
\hline & & & \\
\hline & & & \\
\hline & & & \\
\hline & & & \\
\hline & & & \\
\hline & & & \\
\hline & & & \\
\hline & & & \\
\hline & & & \\
\hline & & & \\
\hline & & & \\
\hline & & & \\
\hline & & & \\
\hline & & & \\
\hline & & & \\
\hline & & - & \\
\hline
\end{tabular}




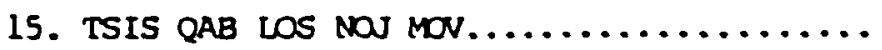
Poor appetite

16. TSIS TUAJ DAB NIUB LOS YOG TSAUG ZOG

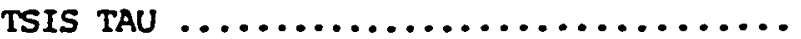
Difficulty falling asleep, staying asleep

17. TSIS MUAJ SIAB VAM RAU YAV TOM NTEJ...... Feeling hopeless about the future

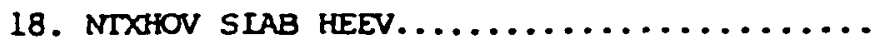
Feeling blue

19. K\$O KHO SLAB IB LEEG................ Feeling lonely

20. TSIS XAV MUAJ TXOU SIA MUS NIXIV........ Thoughts of ending your life

21. NOOG 200 LI YUS WUB NEEJ NYOB HAUV LUAG

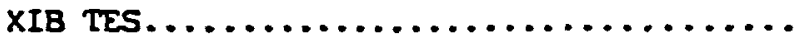
Feeling of being trapped or caught

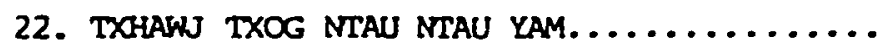
Worrying too much about things

23. TSIS QAB SIAB RAU YAM TWG LI........... Feeling no interest in things

24. XaV tIas yam THG LOS YUAV TAU SIV LUB zOG

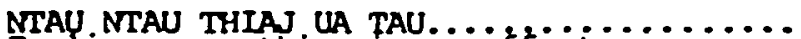
Feeling everything is ar éf foirt

25. NOOG 200 LI YUS TUS KHEEJ TSIS MUAJ NQIS Feelings of worthlessness

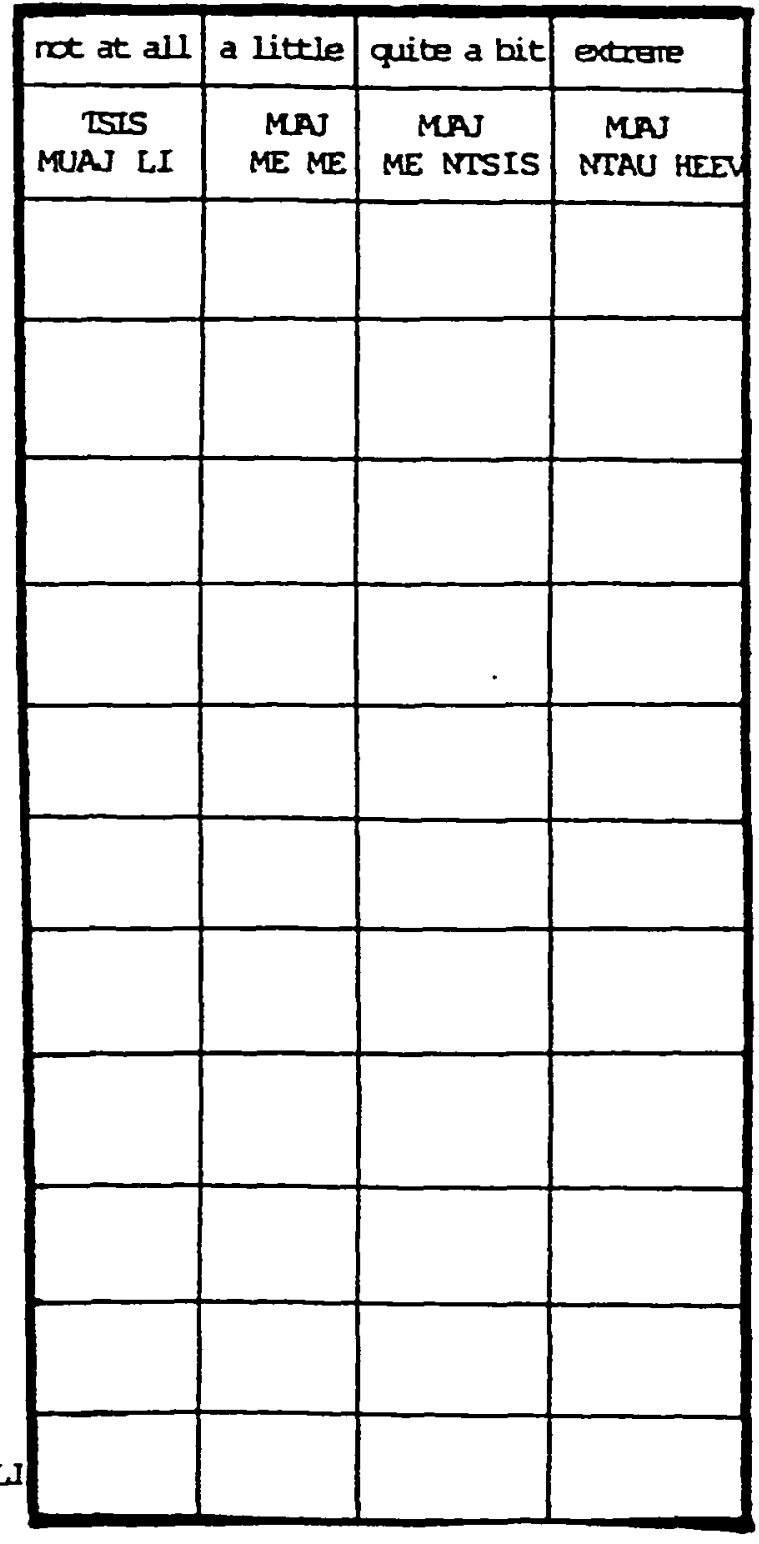

Date:

ID :

Gender:

Age :

DSM-III R Diagnosis:

Year of arrival in U.S.:

Years of education:

Number of family members:

Occupation in Laos:

Occupation in the U.S.: 


\section{Appendix $\mathrm{H}$}

\section{Health Education Materials from the Department of Health Services}

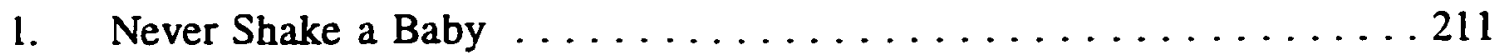

2. Emergency Action for POISONING $\ldots \ldots \ldots \ldots \ldots \ldots \ldots \ldots \ldots$

3. The First Twelve Months: A guide for Infant Feeding . . . . . . . . 214

4. Healthy Teeth for Happy Smiles . . . . . . . . . . . . 216

5. Lead . . . . . . . . . . . . . . . . . . 220

6. Car Safety for Your Child ..................... 221

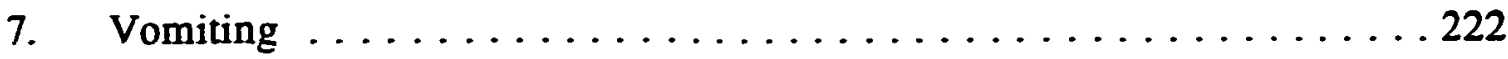

8. Diarrhea . . . . . . . . . . . . . . . . . 223

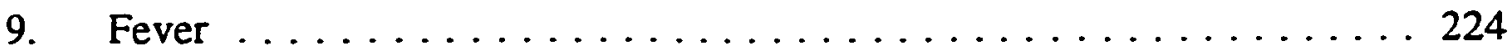

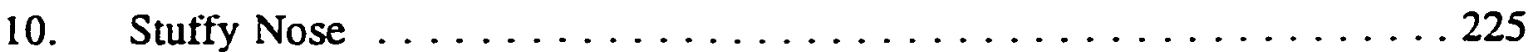

11. Vaccine-Preventable Childhood Diseases ...............226

12. Diphtheria, Tetanus, and Pertussis Vaccine (DPT) . . . . . . . 227

13. Polio Vaccine . . . . . . . . . . . . . . . . . . . . . 229

14. Hepatitis B Vaccine \& Hepatitis B Immune Globulin ............ 231

15. Haemophilus Influenzae $\ldots \ldots \ldots \ldots \ldots \ldots \ldots \ldots \ldots \ldots \ldots \ldots \ldots \ldots$

16. Chickenpox Vaccine ........................ 235

17. Measles, Mumps, and Rubella Vaccine(MMR) ........... 237 


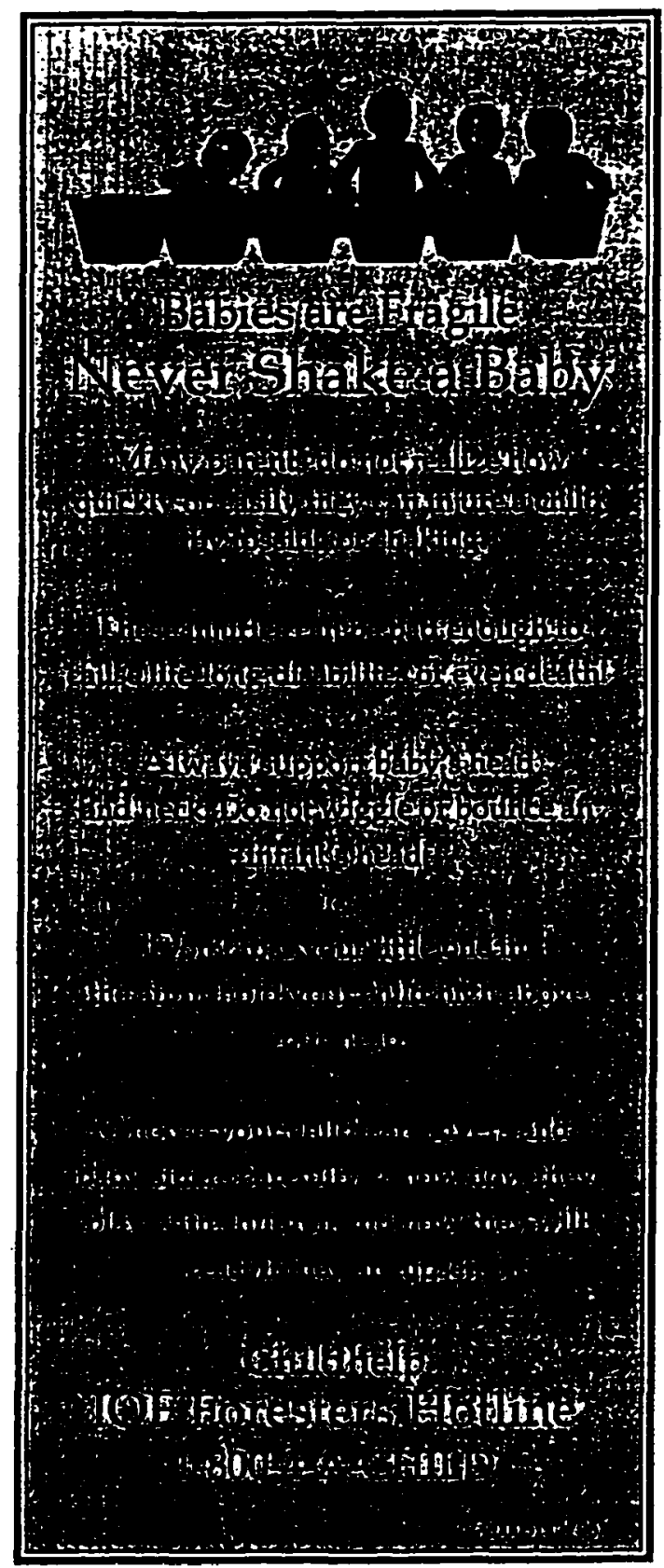




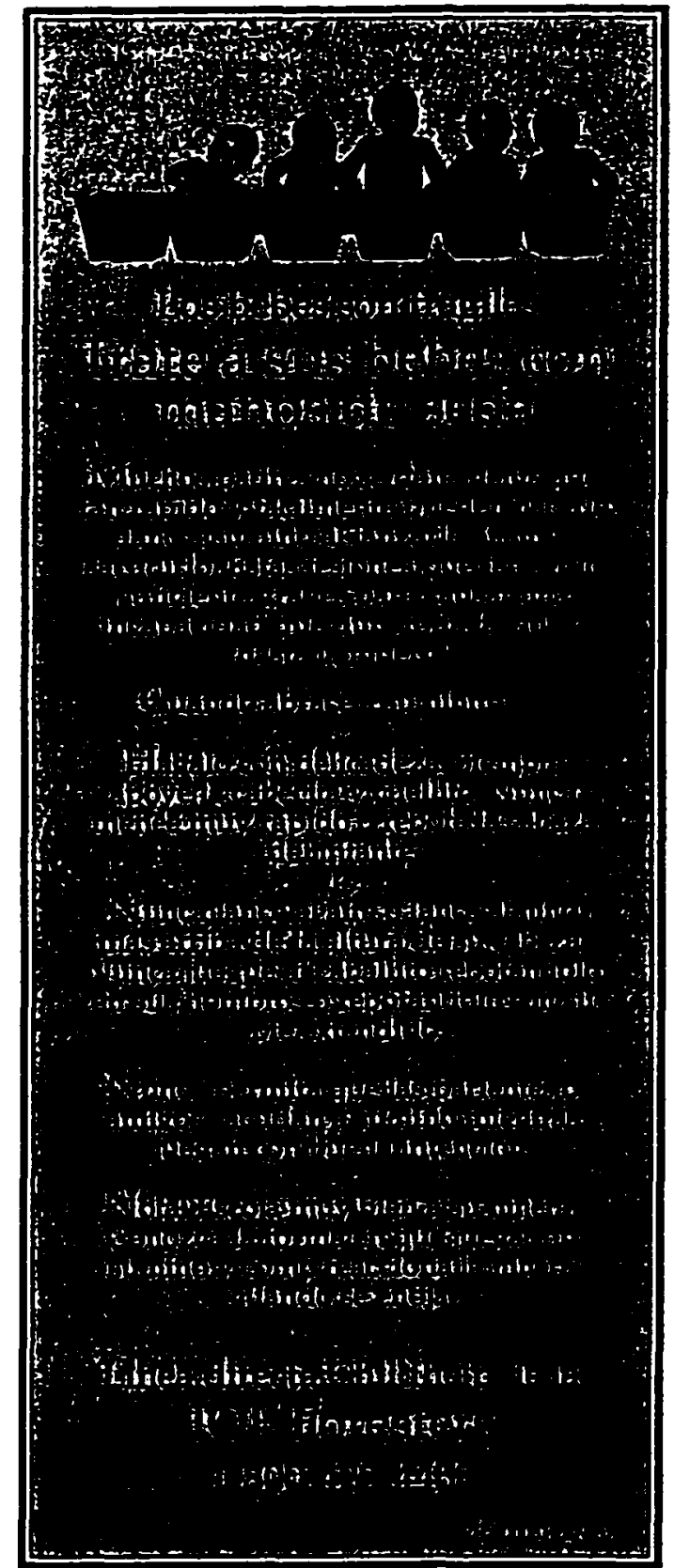




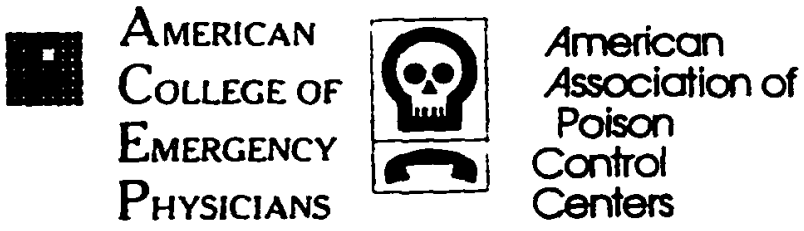

\section{Emergengy Aotion for POISONING}

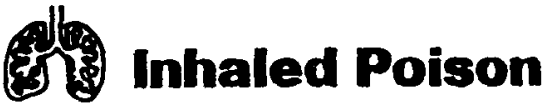

Immediately get the person to fresh air. Avoid breathing fumes. Open doors and windows wide. If victim not breathing, start artificial respiration.

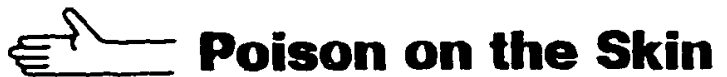

Remove contaminated clothing and flood skin with water for 10 minutes. Then wash gently with soap and water and rinse.

\section{(C) Poison in the Eye}

Flood the eye with lukewarm (not hot) water poured from a large glass 2 or 3 inches from the eye. Repeat lor 15 minutes. Have patient blink as much as possible while flooding the eye. Do not force the eyelid open.

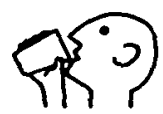

\section{Swallowed Poison}

MEDICINE: Do not give anything by mouth until calling for advice.

CHEMICAL OR HOUSEHOLD PRODUCTS: Unless patient is unconscious, having convulsions, or cannot swallow give milk or water immediately ... then call for professional advice about whether you should make the patient vomit or not.

ALWAYS KEEP ON HAND AT HOME a one ounce bottle of SYRUP OF IPECAC for each child or grandchild at home. Use only on advice of poison control center, emergency department, or physicians.

\section{After the Emergency Actions, CALL}

San Diego Regional Poison Center UCSD Medical Center

\section{1-800-876-4766 or 619-543-6000 Ambulance: Call 911}


Sweeteners such as honey or corn syrups may coniain

intant botullsm Intant

which can lead to death Do

not give these sweeteners babies.

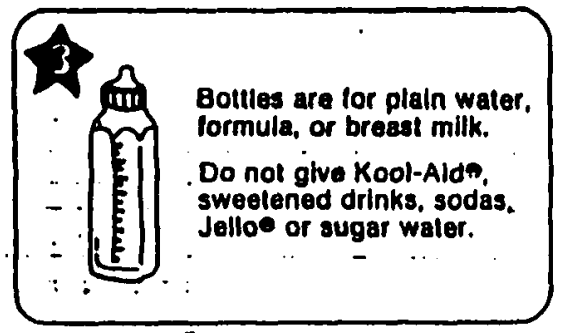

Never lorce your baby 10 Nhish a bolll. Throw away Onyer amall portions ot loolle. Your baby is the best judge of Your baby is the best judge of how much to eat. Throw away

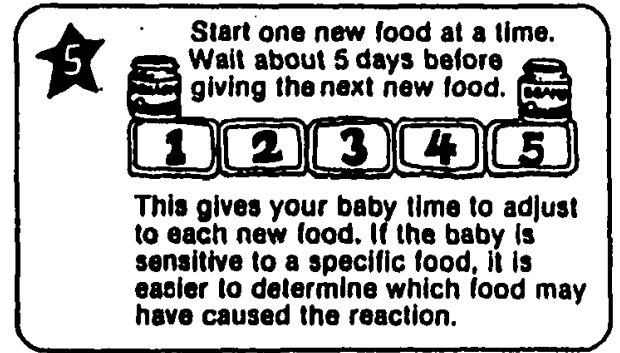
6 Possible allergic
-Skin rash
- Gas
- Respiratory problems -Dlarrhea
Foods that commonly cause allergic reactions include: cow citrus juice citrus truit. gg whiles, wheat products.

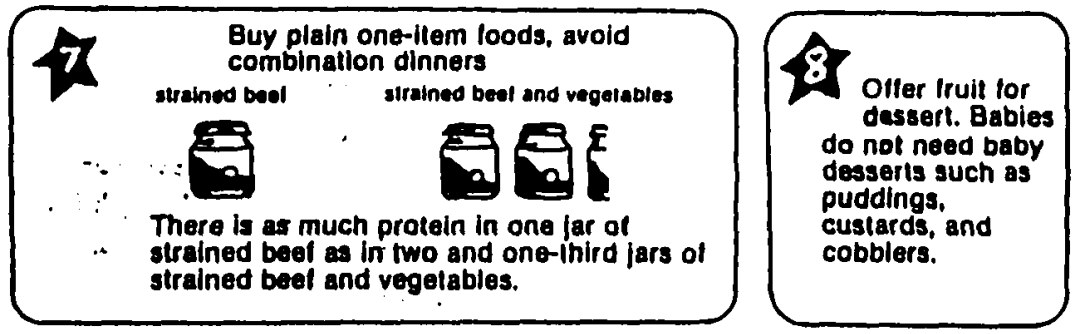

9. Place the amount of food your baby will eal in a small dish. Foed dish. Food leif over in the jar should be tlghily sealed and stored in the reirigeralor.

AOAPTED FAOM VENTUP COUNTY MEALTH DEPAATMENT ANO

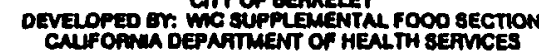

County of 8 an Dieco Deppertment of Herith Senvaes Communitily them Senvicas

P.O. Box 85222 • San Dinco, CA $92180-5220$

\section{The First}

\section{Twelve Months}

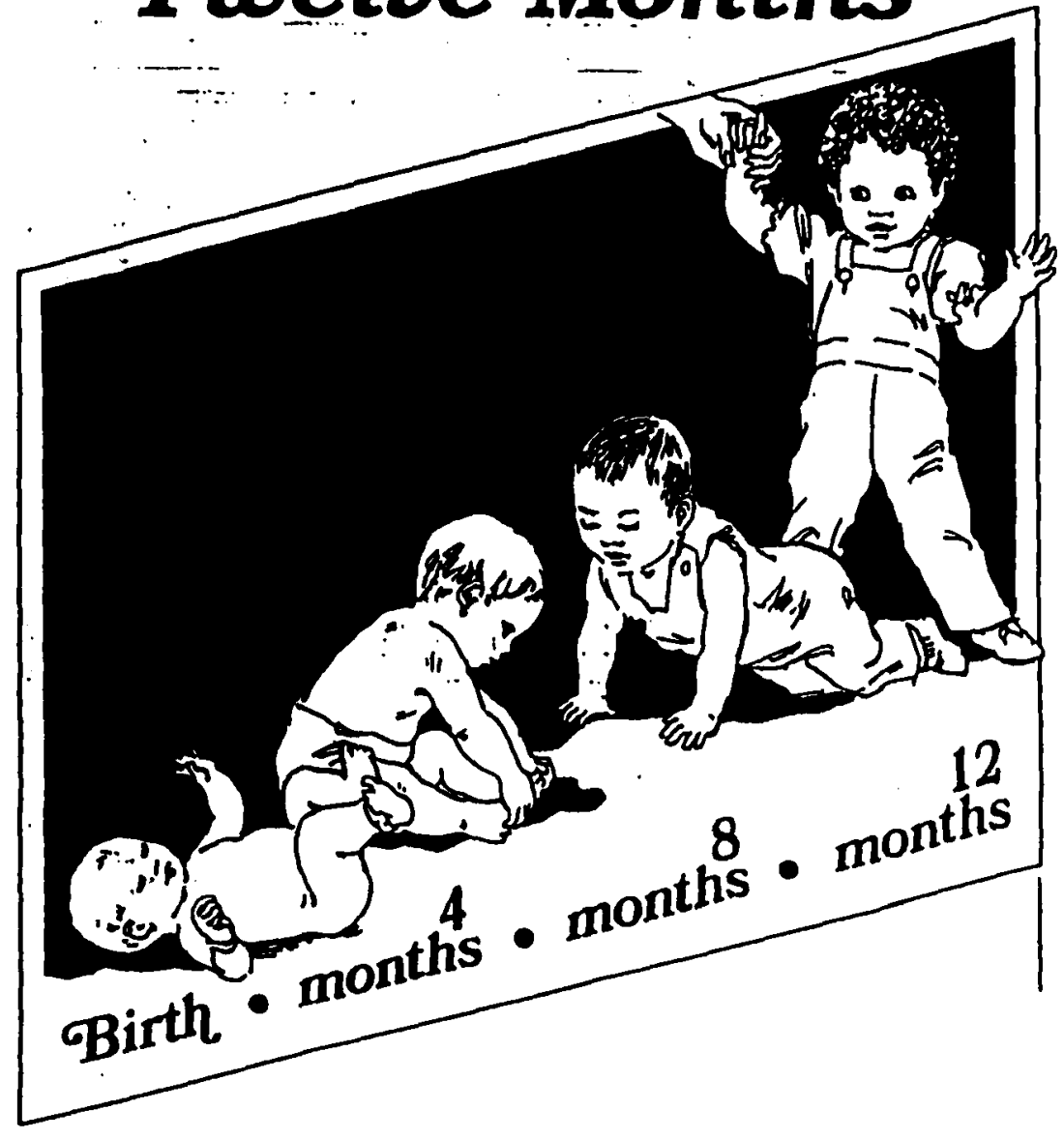

A GUIDE FOR INFANT FEEDING 
The foods that a baby can eat depend on the baby's developmental readiness and

nutritional needs. This guide describes the skills that are usually present at various ages and the foods that the baby is ready to eat

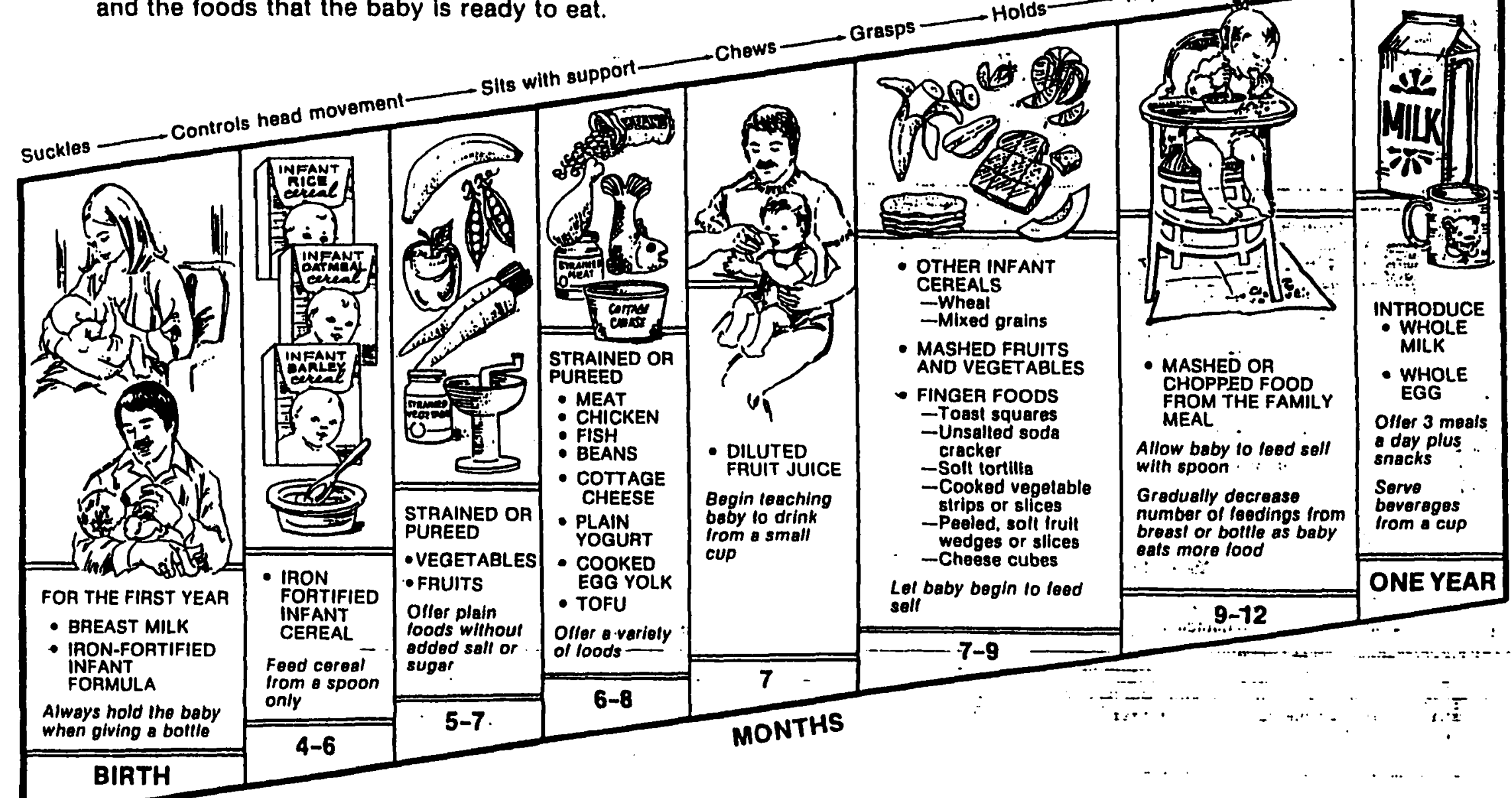

Warning: Babies can easily choke on nuts, seeds, popcorn, raw vegetables, peanut butter, meat sticks and hot dogs. Do not give these foods. Young children should be closely watched when they are eating. 

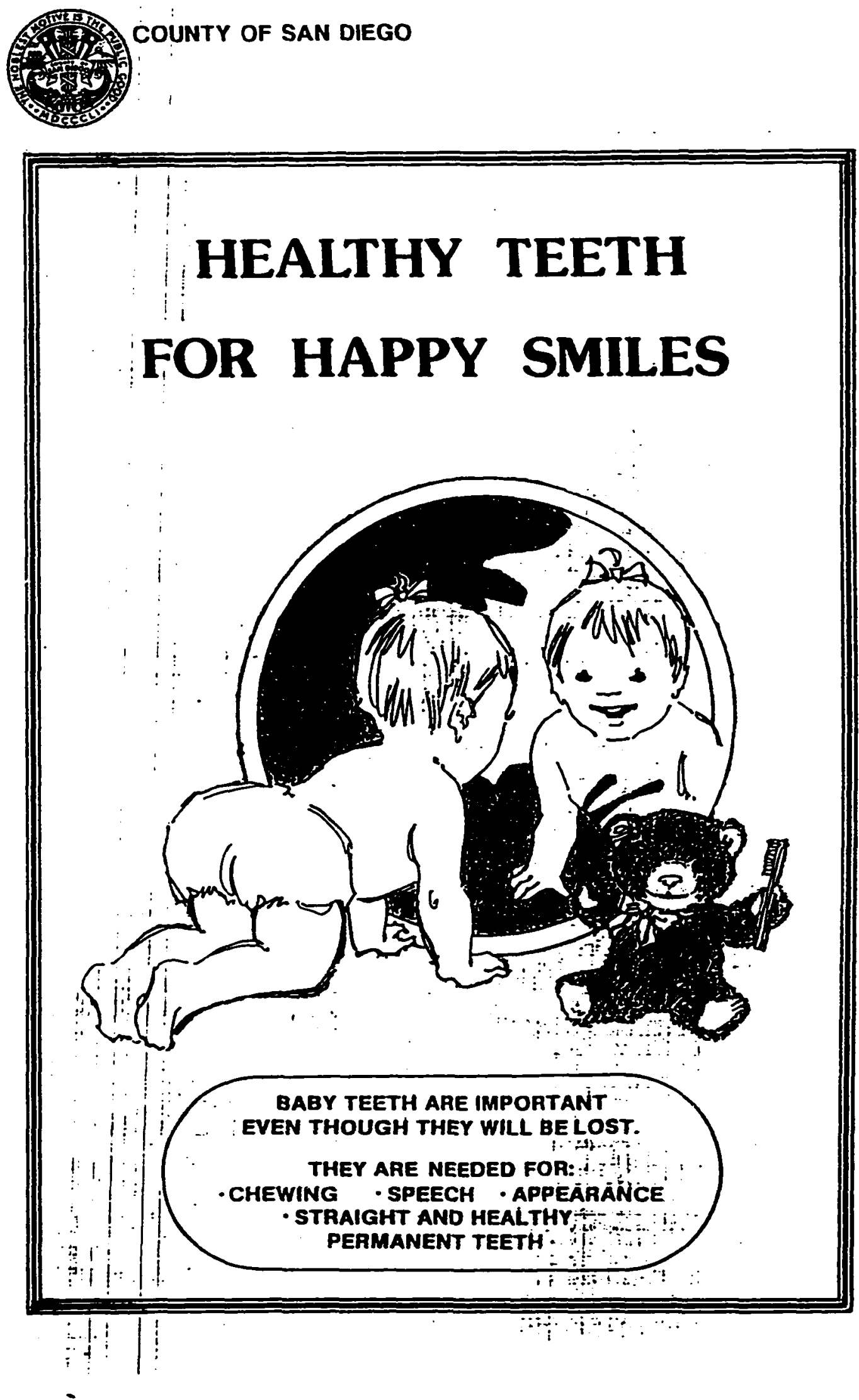


\section{KEEEPING BABS TTEETH HEALTHS}

Proper use of the bottle is the first step in preventing dental problems.

- Bottles are used to feed babies who are not yet able to drink from a cup.

- Feed only formula, breast milk or water from a bottle.

- Offer the bottle only at feeding times. Do not let baby carry a bottle around at other times. A bottle is not a toy or pacifier.

- Sleeping times are not feeding times. Do not put baby to bed with a bottle.

\section{THE BEDTIMAE BOTTLE}

The baby who goes to bed with a bottle can get painful tooth decay. This is called "nursing bottle mouth."

- The sugar in formula, milk, juice and sweetened drinks can decay the teeth if it stays in baby's mouth during sleep.

- Offer a blanket, stuffed animal or favorite toy instead of a bottle at sleeptime.

\section{IT'S TIMAE FOR A CUP}

When your baby is able to sit well, begin offering water from a small cup.

- Be. patient ... it will take your baby time to learn to dritik from a cup.

- As baby gets used to drinking

from a cup, offer formula.

breast milk or juice in a cup.

- T:ake bottles away gradually. Most babies will not want to give up the bottle all at once.

Babies should be drinkíng from a cup by their first

i. birthday.

By eighteen months of age,

If: the bottle should no longer be

! Usedif.

: 


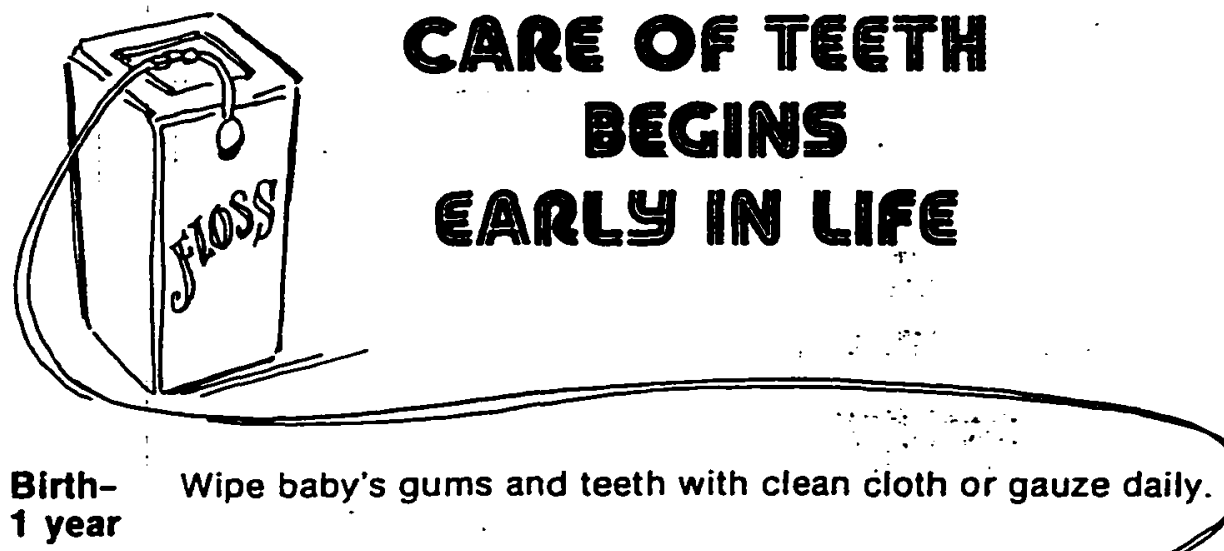

1-2 Gently brush child's teeth with a soft toothbrush daily. years

2-3 Brush and floss child's teeth daily. Begin to teach child to years : brush teeth. Take child for the first dental visit.

3-6 Help child brush and floss teeth daily Take child to the

years Identist regularly.

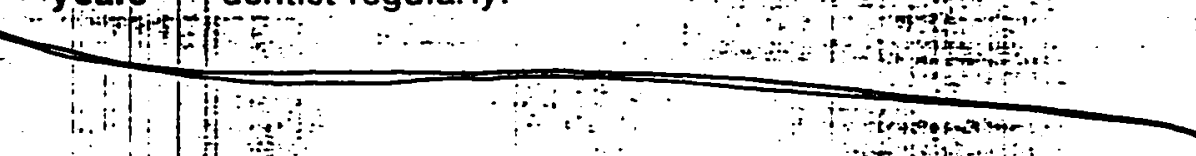

6+ Remind child to brush and floss teeth daliy. Éncourage

years: child to brush teeth after eating sweet or sticky foods. Take

" child to the dentist regularly. 


\section{GOOD NUTRITION FOR HEALTHS TEETH}

\section{Nutritious foods and beverages are needed for healthy teeth and gums.}

- Encourage good eating habits. Choose a variety of foods from each of the food groups. Set regular meal and snack times.

- When your child is thirsty, offer water. Avoid sweet drinks such as soda pop, Hi-C $\odot$. Kool-aid $\odot$. Tang ${ }^{\oplus}$ and fruit punch.

- Help your child control a "sweet tooth." Avoid candy, cookies, cake, pastries. Jello॰. doughnuts, granola bars and baby desserts.

- Brush after eating these foods which stick to the teeth:
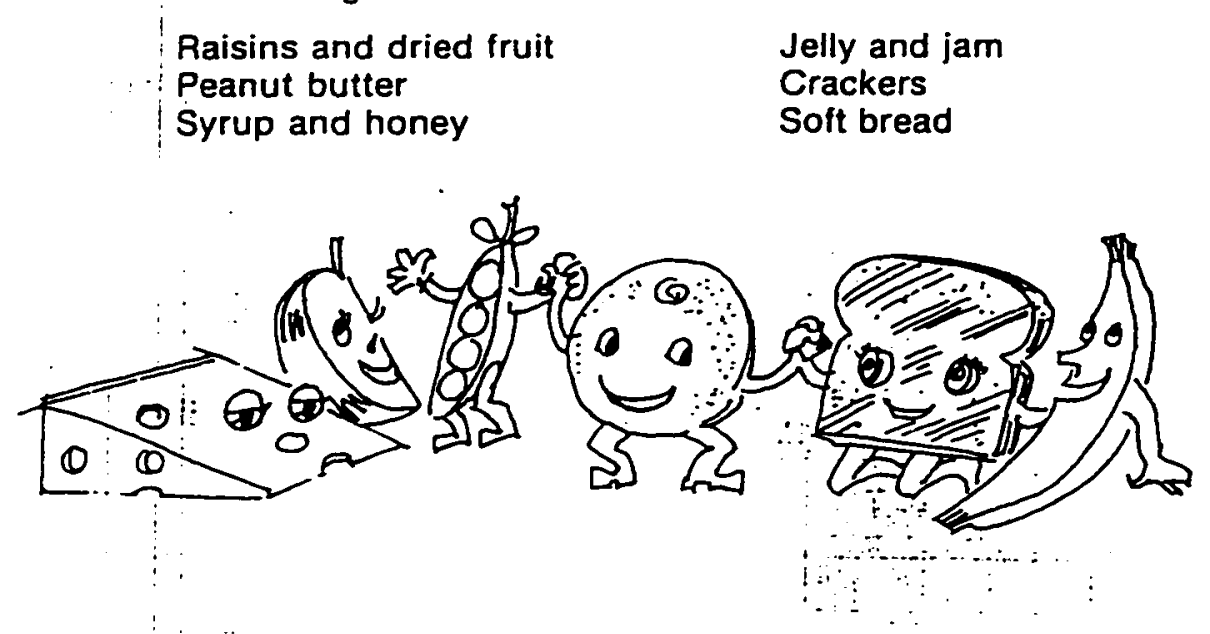

Reprinted courtesy of the Women, Infants and Children (WIC) Supplemental Food Program, California State Department of Health Services.
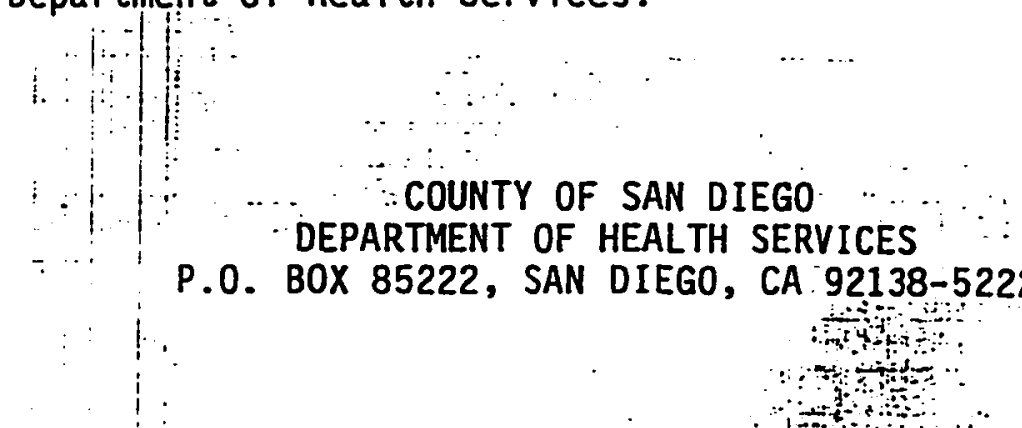

HE P242 (7/88) 30M

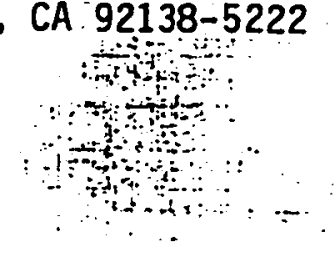



your child away from old paint. Mop and wet-wipe floors and furniture often.

Soil

Pottery

Home Remedies
Find play areas for your child that are not near a busy street, highway, factory or auto shop. Cover soil around your house with plants, rocks or pavement. Wash your child's hands before eating and after playing.

Test ceramic pots, dishes. cups and china for lead. Plastic and glass cups and dishes as well as cast iron and metal pots are safe to use.

Azarcon, greta and others may contain lead. Only give your children doctorapproved medicines.

\section{There are other sources of lead as well. For more information, call the Childhood Lead Poisoning Prevention Program at 236-2643.}

\section{Other ways to protect your child}

\section{Testing}

$\checkmark$ Children between six months and six years of age need to be tested for lead. See your child's doctor for a blood test for lead.

To find out if your child qualifies for a nocost health exam, which includes a blood lead test, call Child Health and Disability Prevention at 692-8428 or 1-(800) 794-3446 (North County).

\section{Diet}

Regular meals and snacks with foods high in calcium and iron, and low in fat will help protect your child from lead.

High-calcium foods: dairy products, tofu. leafy green vegetables, com tortillas.

High-iron loods: lean meats, fortified cereals. dried beans, leafy green vegetables, dried fruits. Low-fat foods: fruits, vegetables, pasta. rice, nonfat dairy products, dried beans and lean meats.

DHS:PHE-P399ES (295) 5M

County of San Diego - Department of Health Seroices

P.O. Box 85222 - San Diego, CA $92186-5222$ 
County of San Diego

\section{Car Safety for Your Child}

Children need to learn certain things for their own safety. Buckling up when getting into a car is one of them. You can help keep your child safe by doing the following:

A Use a car seat that fits your child's age and weight. as well as your car.

A Start early - your newborn baby should come home from the hospital in a federally approved car safety seat.

A Do not use a rear-facing safety seat in a vehicle seat that has an air bag.

A If the vehicle has motorized seat belts, use the safety seat in the rear seat of the vehicle.

A Use the right kind of safety seat at the right age. Choose a seat that helps your toddler or preschooler see out the window.

\section{Weight Guide for Child Safety Seats}

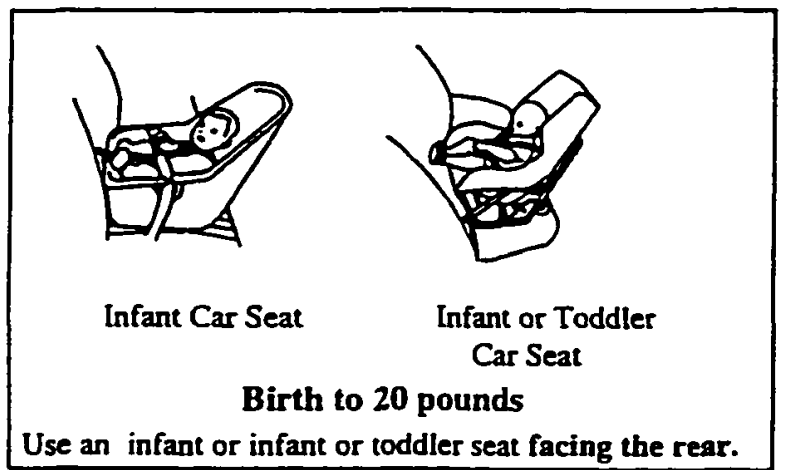

A Set an example. Make sure you and your child always buckle up. even if you only go a few blocks.

A As your baby becomes a toddler, explain in simple words what's good about buckling up. Use a doll to show your child what happens in a car crash.

A Keep your child busy with a bag of travel toys, books, coloring books, small cars, and dolls. Children are less likely to fuss when they have something to do.

A Be firm-if your child unbuckles the belt, stop the car every time and rebuckle it.

A Do not let your child ride in the back of a truck or station wagon.
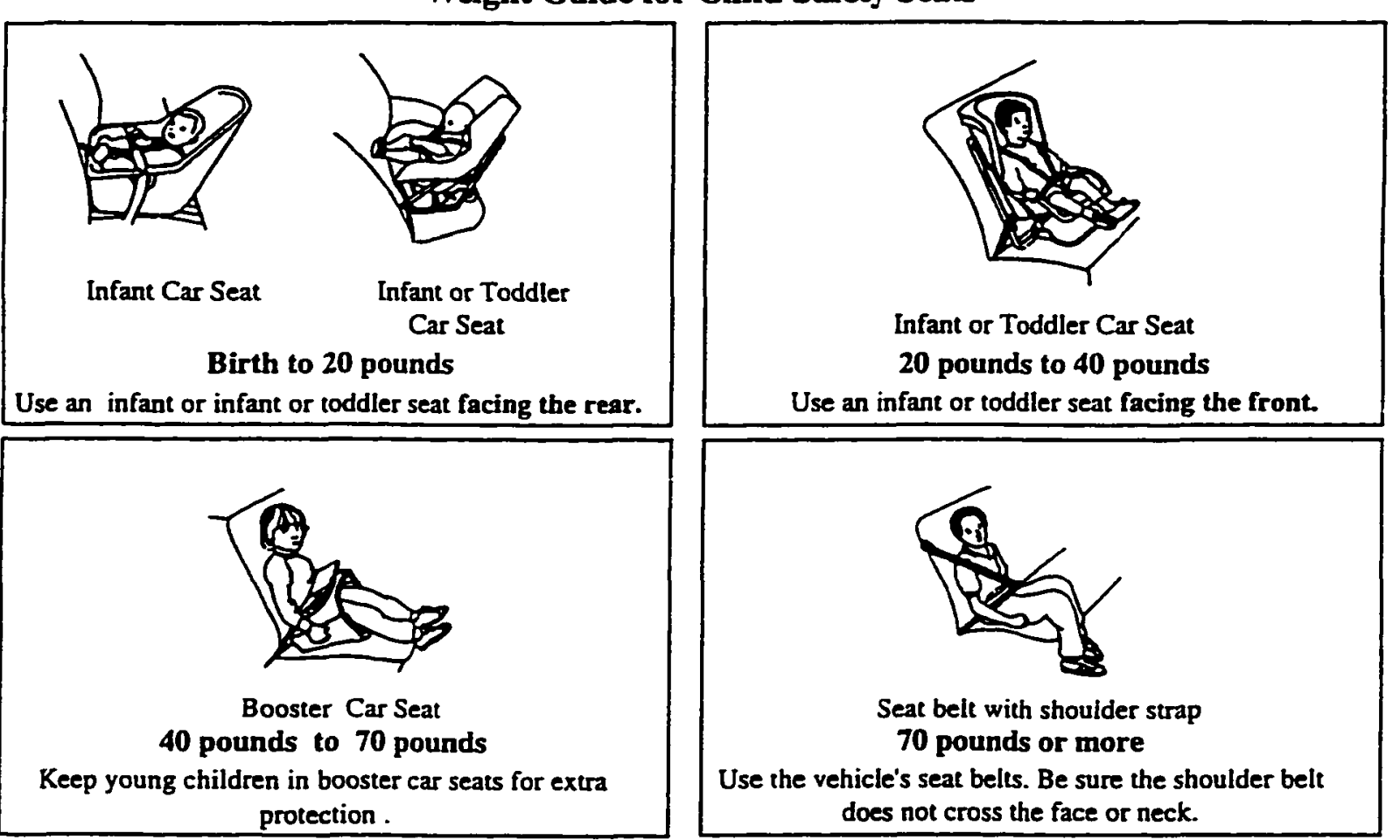

You can also help keep your children safe by
not smoking in the car. This protects them
from breathing unhealthy air.
County of San Diego - Department of Health Services
P.O. Box 85222 - San Diego. CA $92186-5222$

DHS: CHP-P352 ES (6/96) 2M
(Espanol al dorso) 


\section{COUKTY OF SAN DIEGO}

\section{DIARRHEA}

If your child is younger than 1 year old, you should call his/her doctor for information on how to treat the diarriea. Improper treatment of diartiea in an infant can lead to dehydration. Wich is very serious at this age.

1. If your child has diarrhea, with or without vomiting, do not give:

dairy products

citrus or other fruits

any medication (unless ordered by doctor)

beans

2. When your child has diarrhea, you may give:

gelatin

gelatin water made with twice the amount of water required

carbonated drinks stirred to go flat

frozen pop sticks (not for an infant)

water. rice water (no more than one bottle of each a day for infants)

3. When diarrhea begins to improve, you may add:

soup (not creamed or fatty; not tomato)

applesauce

tortillas (no butter)

soda crackers

bananas

toast and jelly

rice

pretzels

dry sweetened cereals

4. When your child's stools are firm, you may add other foods to the diet. These are:

broiled chicken

lean beef

apples (no skin)

cottage cheese

tapioca

yogurt (plain)

5. If your child's stools continue to be firm after the addition of these foods, you may gradually return to the regular diet. Milk should be one of the last foods added. Keep in mind that most episodes of diarrhea in children take at least three to five days for recovery.

\section{KEEP THIS GUIDE AS A REMINDER AT HOME}

COUNTY OF SAN DIEGO DEPARTMENT OF HEALTH SERVICES

P.0. BOX 85222. San Diego, CA 92186-5222

HE P53ES (3/92) 2M

(Español al dorso) 

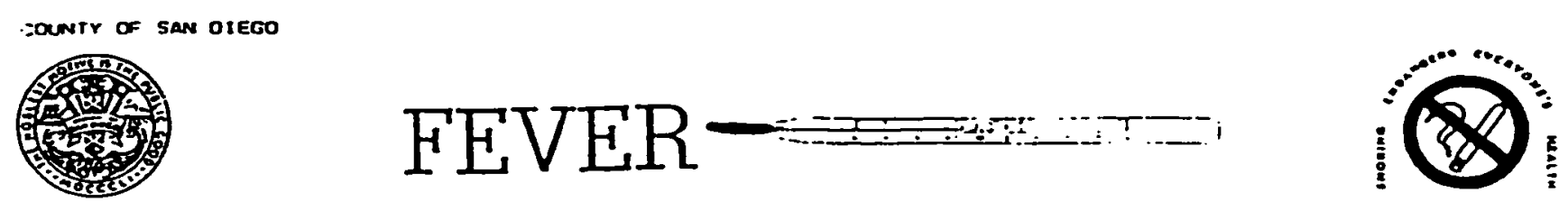

\section{WHEM YOUR CHILD IMS A FEVER:}

1. Give a liquid diet such as juices. water, soda pop (stir it severa) times to let the bubbles out before giving it to the babyl or Jello water ( 4 cups water per small package Jello)

2. Have the child soak for 15 minutes in a tub of warm water to which you slowly add cooler water. Stay with your child. Dry the child well afterwards to avoid shivering.

(NOTE: Do not use alcohol in the bath water to try to lower your child's fever. Alcohol in large amounts or concentrated form in the bath water is now known to be dangerous.)

3. Dress your child in lightweight clothing (cotton shirt and diaper for an infant). Overdressing or bundling just keeps the fever in the child.

4. Use medicine only as directed by the doctor

If your child is under 6 months of age and has a fever of $100.5^{\circ} \mathrm{F}\left(38^{\circ} \mathrm{C}\right)$ or more, call the doctor.

If your child is over 6 months of age and methods of reducing fever fail to bring the temperature below $101^{\circ} \mathrm{F}\left(38.5^{\circ}\right)$, call the doctor.

Special Instructions:

KEEP THIS GUIDE AS A REMIIIDER AT HOME

COUNTY OF SAN DIEGO DEPARTMENT OF HEALTH SERVICES

P.0. Box 85222, San Diego, CA 92186-5222

HE PATES (8/89) $2 M$

(Español as dorso) 


\section{Stuffy Nose}

\section{Stuffy noses are common even in little babies.}

Liule babies cannot breathe through their mouths as adults can, uniess they are crying. Because babies cannot blow their noses. it can be very difficult for them to breathe when the nose is stuffy. You can keep your baby's nose clean so breathing, eating. and sleeping are easier.

Tissues or q-tips and cotoon only wipe the surface. They don't clean out the inside of the nose. $\wedge$ sucrion bulb will solve the problern.

The nose should be cleaned whenever it secms stuffy. especially just belore feeding or siceping.

Note: Some people clean a baby's nose by blowing through the baby's mouth with their lips. This should be done only in emergencies. Otherwise doing this could cause damage to the baby's lungs.

$\Lambda$ suction bulb may look like this

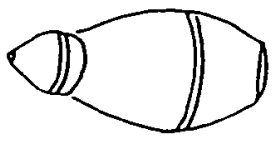

or like this

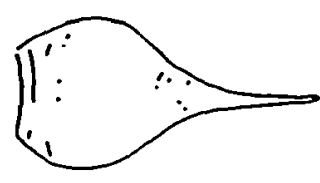

The suction bulb is made entirely of rubber and may be any color.

To use a suction bulb, follow these instructions:

1. Place baby on back and have someone hold your infant so baby can't wiggle head or hands. (If no one else is around. place baby on a sheet on the floor and gently sieady baby's head between your knces.)
2. Squecze the suction bulb before pulling it into baby's nose.

3. While still squeezing. put the tip of the bulb into the baby's right nostril. pointing it straight down.

4. Put a finger over the baby's left nostril and quickly release (stop squecing) the bulb so that it sucks mucus out of the right nostril. Take bulb and both your hands away from the baby and quickly squeeze bulb over a tissue to get the mucus out of the bulb.

5. Carefully repeat this procedure with the left nostril.

Read this again so you can show the nurse how to do it.

Clean the suction bulb with warm, sudsy waler after each use and rinse it several times with elean water. Try to force all excess water out of it

If you don't have a sucrion bulb, buy one today.

Babies with a stuffy nose need to see the doctor when:

1. They are having trouble breathing or are breathing very rapidly even after you ve carefully cleaned out the nose.

2. They are drinking much less than usual.

3. Tiey seem to ery each time you move them or

4. They have to be awakened for several of their usual foedings.

The suction bulb should not be used for the ear unless specifically advised by the docior.

Keep this guide as a reminder at home.

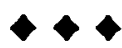




\section{Vaccine-Preventable Childhood Diseases}

\section{mumps}

- causes fever, headache and inflammation of the salivary glands, resulting in swelling of cheeks or jaw

- one out of every 10 who get mumps may develop meningitis, sometimes causes encephalitis

- can result in permanent loss of bearing

- MMR vaccine prevents this disease

rubella

- slso known as german measles

- most serious in pregnint woman; there is an $80 \%$ percent chance that it will cause defects in the unborn child if infection occurs in pregnancy

- symptoms inchude mild discomfort, slight fever for 24 hours, and a rash on the face and aeck lasting for two or three days

- MOMR vaccine prevents this disease

haemophilus influenzae type $b$

- also known as Hib disesse

- strikes one child out of 200 before the fifth birthday

- more serious in infants under one year of age

- causes pneumonia and infections of the blood, joints, bones, soft tissues, throat and the covering of the heart

- causes meningitis in about 12,000 children per year, about one in four suffers permanent brain damage, and about one in 20 dies

- Hib vaccine prevents this disease

hepatitis b

- infints born to HBV-infected mothers have up to an $85 \%$ chance of getting the infection

- HBV infection is spread within families and communities

- infection at birth or during early childhood can result in longterm chronic illness

- chrouically infected persons can develop chronic liver disease and a rare form of cancer

- HBV vaccine prevents this disease polio

- serious cases cause paralysis and death

- mild cases cause fever, sore throat, nausea, headaches, stomach aches; stiffiness in the neck, back and legs also occurs

- can be prevented with the oral polio vaceine or inactivated polio vaccine

varicella

- also known as chickenpox

- causes a blister-like rash, itching, tiredness and fever

- complications can include pneumonia brain damage and death

- persons who have had chickenpox can develop shingies years later (shingles causes a painful skin rash)

- varicella vaccine prevents this disease diphtheria

- easily spread through coughing or sneezing

- early symptoms are sore throat, slight fever, and chills

- can intefere with swallowing and cause suffocation

- can cause heart failure or paraiysis if allowed to go untreated

- can be prevented with DTP vaccine

tetnnus

- also known as lockjaw

- enters the body through a wound

- produces a poison which affects the body's nervous systerr.

- symptoms are headacbe, irritability, stiffiess in jaw and neck

- causes muscle spasms in the jaw, neck, arms, legs and

abdomen

- may require intensive hospital care

- 3 out of every 10 people in the U.S. who get tetanus die

- can be prevented with DTP vaccine

pertussis

- also known as whooping cough

- highly contagious

- camses severe spells of coughing which can interfere with eating, drinking and breathing

- complications may include preunnonia, convulsions, or encephalitis

- in the U.S. about $65 \%$ of reported cases occur in children under 5 years, and half of those are infants less than 1 year old

- in recent years, an average of 3,500 cases reported in the U.S.

- can be prevented with DTP vaccine - i:1:; measles

- highly contigious

- causes a mash, bigh fever, cough, runny nose, and watery

eyes, lasting for one to two weeks

- causes ear infections and pneumonia in one out of every 20 children who get it

- causes encephalitis that can lead to convitsions, deafness or mental retardation in one out of every 1,000 children who get it

- of every 1,000 children who get measies, one or two will die

- MMR vaccine prevents this disease

\section{Baby Shots Line 692-6600}

to review your baby's Immunization record 


\section{IPHTHERIA,} ITANUS, ND

:RTUSSIS ICCINE TP)

you need to know e your child gets accine
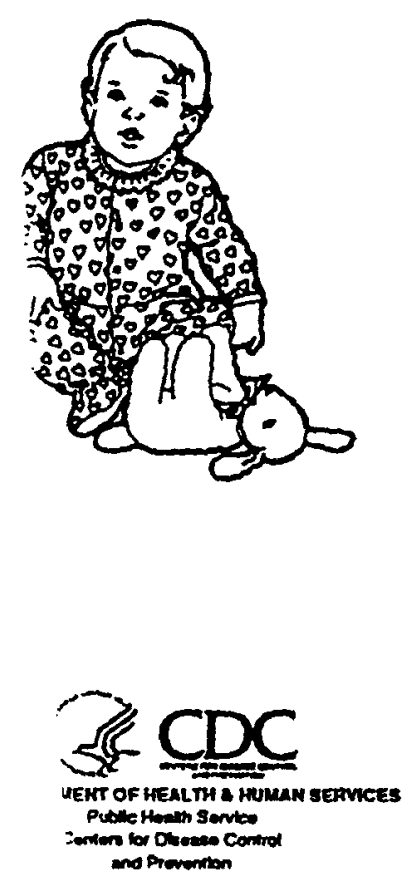

\section{ABOUT THE DISEASES}

Diphtheria tetanus (lockjow). and pertussis (whooping cough) are serious diseases. Diphtheria and pertussis spreod when germs pass from an infected person to the nose or throat of others. Tetanus is coused by a germ that enters the body through a cut or wound.

\begin{tabular}{|c|c|c|}
\hline $\begin{array}{l}\text { Dlphitherda causes: } \\
\text { o thick coating in } \\
\text { the nose. throat. or } \\
\text { girwoy }\end{array}$ & $\begin{array}{l}\text { Tetanus causes: } \\
\text { sertous. painful } \\
\text { spasms of all } \\
\text { muscles }\end{array}$ & $\begin{array}{l}\text { Pertussis couses: } \\
\text { coughing and } \\
\text { choking for several } \\
\text { weeks (mokes it } \\
\text { hard for infonts to } \\
\text { eat. drink. or } \\
\text { breathe) }\end{array}$ \\
\hline $\begin{array}{l}\text { it can lead fo: } \\
\text { - breathing problems } \\
\text { - heart failure } \\
\text { - poratysis } \\
\text { - death }\end{array}$ & $\begin{array}{l}\text { It can lead to: } \\
\text { - "locking" of the fow } \\
\text { so the patlent } \\
\text { connot open his or } \\
\text { her mouth or } \\
\text { swallow } \\
\text { - death }\end{array}$ & $\begin{array}{l}\text { It can lead to: } \\
\text { - pneumonio } \\
\text { - seizures Jerking } \\
\text { and staring spelis) } \\
\text { - broin damage } \\
\text { - death }\end{array}$ \\
\hline
\end{tabular}

\section{Benefits of the vaccines \\ Related vaccines}

Vaccination is the best way to protect ogoinst diphtheria. tetanus, and pertussis. Becouse most children get the vaccines. there are now many fewer cases of these diseases. There would be many more cases if we stcpped voccinaling children.

\section{DTP schedule}

Most children should have a total of 5 DTP voccines. They should hove DTP at:

$\checkmark 2$ months of age

$\checkmark 4$ months of age

$\checkmark 6$ months of age

$\checkmark 12-18$ months of age

$\checkmark$ 4-6 years of age

\section{DTaP (Diphtheria Ielanus} gcellular Pertussis)

- Uke DTP. it prevents diphtheria. tetanus. and pertussis.

- It is oniy given for the ath and 5ith doses.

- It is less likely to couse the mild problems we see offer DTP and is probably less likely to cause some of the moderate problems.

DT (Diphtheria Ietanus)

- Unilke DTP, it does not prevent pertussts. For this reason. It is usually not recornmended.

Other vaccines moy be given at the same time os DTP.

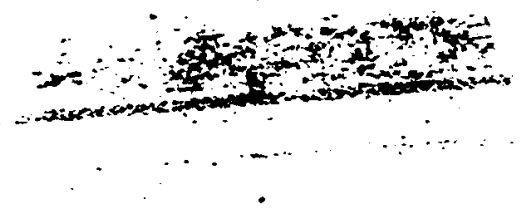

permission of the copyright owner. Further reproduction prohibited without permission. 


\section{Who should get DIP vaccine?}

Most doctors recommend that aimost all young children get DIP or DTaP vaccine. Some children should get DT. With all vaccines. there are some coutions.

Tell your doctor or nurse if the child getting the vaccine:

- ever had a serious allergic reaction or other problem after getting DIP. DTaP. or DT

- now has moderate or severe illness

- has ever had a selzure

- has a parent, brother. or sister who hos had sebures

- has a brain problem that is getting worse

If you are not sure. ask your doctor or nurse.

\section{What are the risks from these vaccines?}

As with any medicine. there are very small risks that sertous problems, even death. could occur after getting a vaccine.

The risks from the vaccine are much smaller than the risks from the diseases if people stopped using vaccine.

Below is a list of problems that may occur after getting the vaccine. If your child ever had one of the moderate or severe problems listed below or any other serious problem affer DTP. DTaP, or DT. dlscuss it with your doctor or nurse before this vaccination.

\section{Mild problems}

If these problems occur. they usually start within hours to a day or two after vaccination. They usually last up to 1-2 doys:

- soreness, redness, or swelling where the shot was glven

- fever

- fussiness. drowsiness. less appetite

Acetaminophen or lbuprofen (non-aspirin) may be used to prevent or reduce fever and soreness. This is especlally important for children who hove had selzures or have a parent, brother, or sister who has had selzures.

\section{Moderate problems}

Once for every $100-1.000$ doses:

- on-going crying for 3 hours or more

- fever of $105^{\circ}$ or higher

- an unusual. high-pitched cry

Once for every 1.750 doses:

- a selzure (jerking and staring spell) usually coused by fever

- "shock-collapse" (becomes pale. limp. and less alert)

\section{Severe problems}

These problems happen very rarely:

- serious allerglc reaction offer DT or DTP

- a long seizure

- decreased consclousness or coma. Some of these children moy hove losting brain damage. There is dlsagreement about whether or not DIP causes the lasting brain damage. If it does. it is very rore.

\section{What to do if there is a serious reaction:}

Call a doctor or get the person to a doctor right owoy.

Les Write down what happened and the date and time it happened.

Ask your doctor. nurse. or health department to flle a Voccine Adverse Event Report form or call:

$$
\text { (800) 822-7967 (toll-free) }
$$

The National Vaccine Injury Compensation Program gives compensation (payment) for persons thought to be injured by vaccines. For detalls call:

$$
\text { (800) 338-2382 (toll-free) }
$$

If you want to learn more. ask your doctor or nurse. She/he can give you the vaccine package insert or suggest other sources of information. 
POLIO

\section{VACCINE}

What you need to know before you or your child gets the vaccine
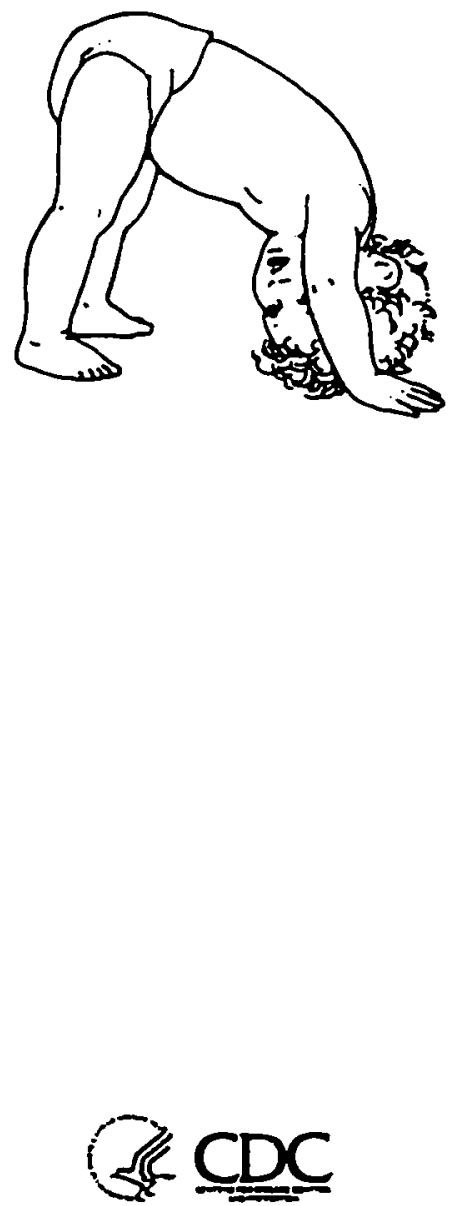

US. DEPNATIENT OF HEALTH \& MUMAN SEAVICES Putele Heen Senves

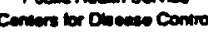
end Prevertion

\section{ABOUT THE DISEASE}

Polio is a serious disease. If spreads when germs pass from on infected person to the mouths of others. Polio can:

- paralyze a person (make arms and legs unable to move)

- cause death

\section{ABOUT THE VACCINES}

\section{Benefits of the vaccines}

Vaccination is the best woy to protect against pollo.

Becouse most children get the polio voccines. there are now very few cases of this disease. Before most children were voccinated. there were thousands of cases of polio.

\section{There are 2 kinds of polio} vaccine

OPV or Qral Pollo Vaccine is the one most often given to children. It is given by mouth as drops. It is easy to give and works well to stop the spread of polio.

IPV or Incctivated Pollo Vaccine is given as a shot in the leg or orm.

\section{OPV schedule}

Most children should have a total of 4 OPV vaccines. They should hove OPV at:

$\checkmark 2$ months of age

$\checkmark 4$ months of age

$\checkmark 6-18$ months of age

$\checkmark$ 4-6 years of age

Other vaccines moy be given at the same time as OPV.

\section{Who should get OPV?}

Most doctors recommend that atmost all young children get OPV. But there are some coutions. Tell your doctor or nurse if the person getting the vaccine or anyone else in close confoct with the person getting the vaccine is less able to fight serious infectlons becouse of:

- a disease she/he was born with

- treatment with drugs such as long-term steroids

- any kind of cancer

- concer treatment with $x$-roys or drugs

- AIDS or HIV infection

If so. your doctor or nurse will probably give IPV Instead of OPV.

If you are older than age 18 years. you usually do not need polio vaccine.

Iravel

If you are troveling to a country where there is polio. you should get either OPV or IPV.

\section{Preanancy}

If protection is needed during pregnancy. OPV or IPV can be used.

\section{Alleray te neemvein or} streptomycin

Does the person getting the vaccine have an allergy to the drugs neomycin or streptomycin? If so. she/he should get OPV. but not IPV. Ask your doctor or nurse if you are not sure. 
Tell your doctor or nurse if the person getting the voccine:

- ever had a serious allergic reaction or other problem after getting polio vaccine

- now has moderate or severe illness

If you are not sure. ask your doctor or nurse.

\section{What are the risks from polio vaccine?}

As with any medicine. there ore very small risks that serious problems, even death, could occur after getting a vaccine.

The risks from the vaccine are much smaller than the isks from the disease if people stopped using vaccine.

Almost all people who get pollo vaccine hove no problems from it.

\section{Risks from OPV}

Risks to the person taking OPV:

There is a very small chance of getting polio

disease from the vaccine.

- about I case occurs for every $l_{2}^{\frac{1}{2}}$ million first doses

- about 1 case occurs for every 30 million later doses

Risks to people who never took polio vaccine who hove close contact with the person taking OFV:

After a person gets OPV, it can be found in his or her mouth and stool. If you never took polio vaccine. there is a very small chance of getting polio disease from close contact with a child who got OPV in the past 30 days. (Examples of close contact include changing diapers or kissing.)

- about 1 case occurs for every 2 million first doses

- about I case occurs for every 15 million later doses

Talk to your doctor or nurse about getting IPV.

\section{Risks from IPV}

This vaccine is not known to couse problems except mild soreness where the shot is given.

\section{What to do if there is a serious reaction:}

Call a doctor or get the person to a doctor right oway.

Write down what happened and the date and time it hoppened.

45s Ask your doctor. nurse. or health department to file a Vaccine Adverse Event Report form or call:

(800) 822-7967 (toll-free)

The National Vaccine Injury Compensation Program gives compensation (poyment) to persons thought to be injured by vaccines. For details call:

(800) 338-2382 (toll-free)

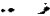

If you want to learn more. ask your doctor or nurse. She/he can give you the vaccine package insert or suggest other sources of information.

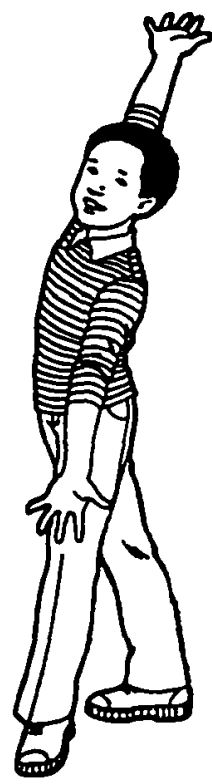




\section{Who should get hepatitis B vaccine?}

1. Most infants

2. Children 10 years of age and younger whose parents are from parts of the world where hepatitis B is common

3. Pre-teens and teens who did not get the vaccine already

4. Adults at risk

Ask your doctor or nurse if you should get the vaccine.

Tell your doctor or nurse if the person getting the vaccine:

- ever had a serious allergic reaction to:

- hepatitis B vaccine

: common baker's yeast .

- currently has a moderate or severe illness

If you are not sure, ask your doctor or nurse.

What are the risks from hepatitis $B$ vaccine?

Hepatitis $B$ vaccine is one of the safest vaccines.

As with any medicine, there is a very small tisk that serious problems, even death, could occur after getting a vaccine.

Getting the disease is much more likely to cause serious illness than getting the vaccine.

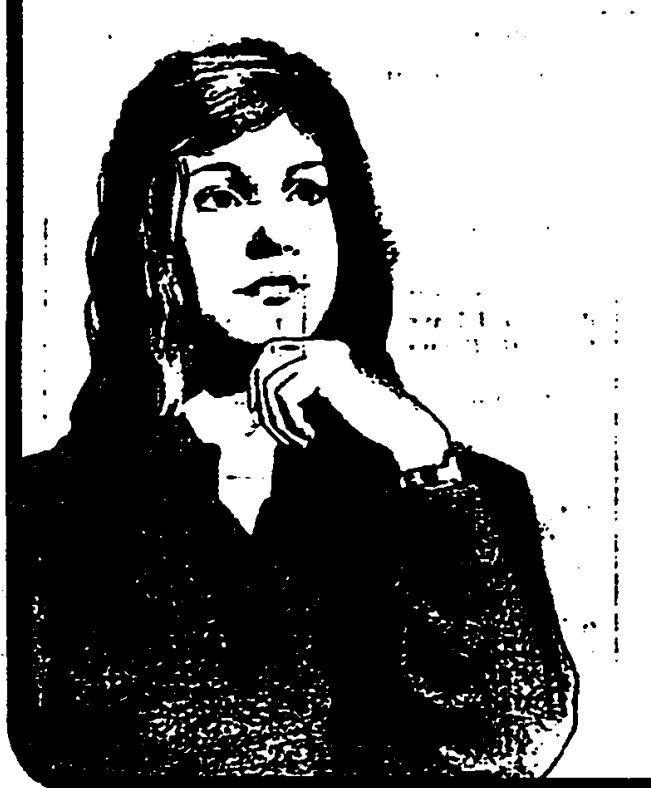

Hep B - 5/1/96

\section{Mild problems}

- soreness where the shot was given

- mild to moderate fever

Acetaminophen or ibuprofen (not aspirin) may be used to reduce fever and pain.

\section{Severe problems}

- serious allergic reaction is very. rare $\because$

What to do if there is a serious reaction:

Les Call a doctor or get the person to a doctor right aiway.

Ask your doctor, nurse, or health department to file a Vaccine Adverse Event Report form. To file a report yourself you can call: - (800) 822-7967 (toll-free)

If you want to learn more, ask your doctor or nurse. She/he can give you the vaccine package insert or suggest other sources of information.

About Hepatitis B Immune Globulin (HBIG)

Benefits: HBIG protects from hepatitis B virus infection for 1-3 months.

Schedule: HBIG is given with the first hepatitis $B$ vaccine dose to people who have recently been exposed to hepatitis $B$ virus.

Who should get HBIG::

- Newborns of women infected with hepatitis B virus

- Infants who never got the vaccine and whose parent or caregiver has hepatitis B

- People recently exposed to blood or body

, fluids that may contain hepatitis B virus

- People who recently had sex with someone i with hepatitis B

R̈isks: swelling, hives, severe allergic reaction 2. 
Who should get hepatitis B vaccine?

1. Most infants

2. Children 10 years of age and younger whose parents are from parts of the world where hepatitis $B$ is common

3. Pre-teens and teens who did not get the vaccine already

4. Adults at risk

Ask your doctor or nurse if you should get the vaccine.

Tell your doctor or nurse if the person getting the vaccine:

- ever had a serious allergic reaction to:

- hepatitis B vaccine

: common baker's yeast .

- currently has a moderate or severe illness

If you are not sure. ask your doctor or nurse.

What are the risks from hepatitis B vaccine?

Hepatitis B vaccine is one of the safest vaccines.

As with any medicine, there is a very small risk that serious problems, even death. could occur after getting a vaccine.

Getting the disease is much more likely to cause serious illness than getting the vaccine.

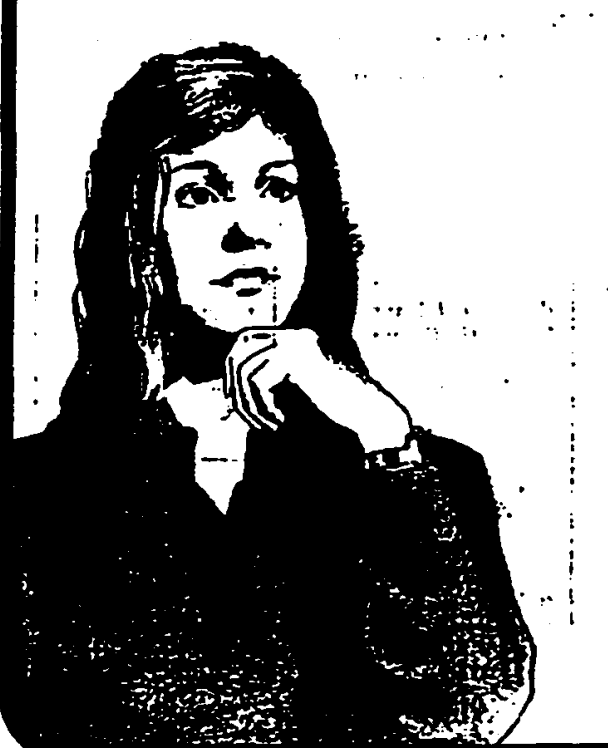

Hep B - 5/1/96
Mild problems

- soreness where the shot was given

- mild to moderate fever

Acetaminophen or ibuprofen (not aspirin) may be used to reduce fever and pain.

Sevare problems

- serious allergic reaction is very. rare

What to do if there is a serious reaction:

Call a doctor or get the person to a doctor right away.

Ask your doctor, nurse, or health department to file a Vaccine Adverse Event Report form. To file a report yourself you can call: - (800) 822-7967 (toll-free)

If you want to learn more, ask your doctor or nurse. She/he can give you the vaccine package insert or suggest other sources of information.

About Hepatitis B Immune Globulin (HBIG)

Benefits: HBIG proiects from hepatitis B virus infection for $1-3$ months.

Schedule: HBIG is given with the first hepatitis $B$ vaccine dose to people who have recently been exposed to hepatitis B virus.

Who should get HBIG: $:$,

- Newborns of woinen infected with hepatitis B virus

- Infants who never got the vaccine and whose parent or caregiver has hepatitis B

- People recently exposed to blood or body

fluids that may contain hepatitis B virus

- People who recently had sex with someone with hepatitis B

Risks: swelling, hives, severe allergic reaction 3 
HAEMOPHILUS INFLUENZAE TYPE b VACCINE (Hib)

What you need to know before:your child gets the vaccine

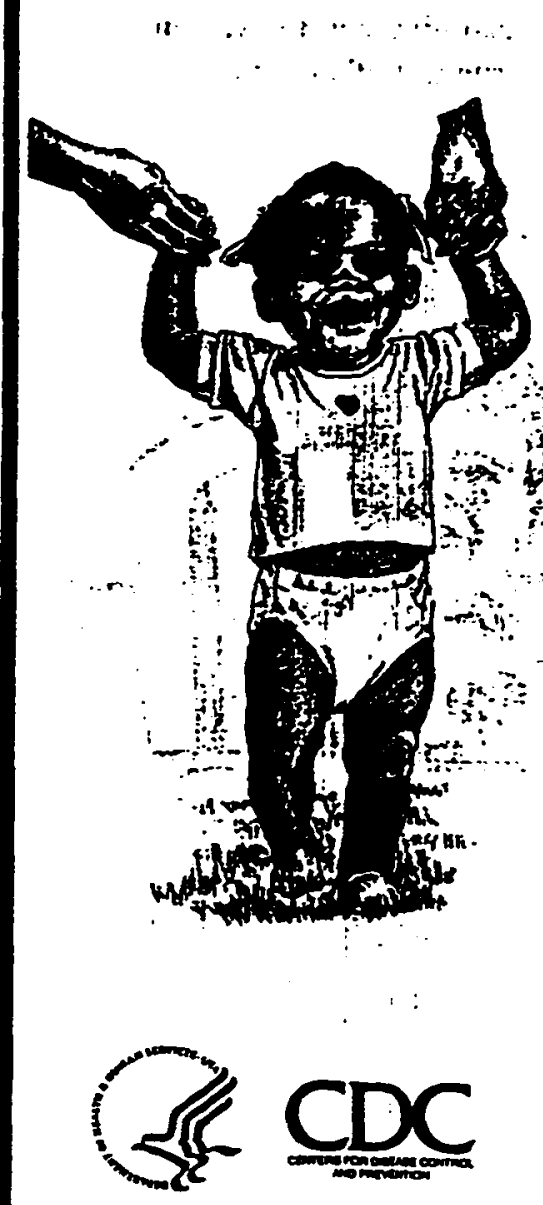

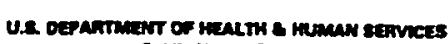
prowe theolith Sorves

\section{ABOUT THE DISEASE}

Haemophilus influenzae

type $b$ (Hib) disease is serious. It is most common in children less than 5 years old.
Hib can cause:

- meningitis (infection of the brain and spinal cord coverings)

- lasting brain damage

- pneumonia

- infections of the blood, joints, bones, soft tissue under the skin, throat, and - the covering of the heart - déáth

\section{ABOUT THE VACCINE}

\section{Benefits of the vaccind Who should get Hib}

Vaccination is the best way to protect against Hib disease. Because most children get the Hib vaccine, there are now" than 5 years of age get Hib. many fewer cases of disease caused by Hib. There would be many $\quad$ Tell your doctor or nurse if more cases if we stopped $\Rightarrow$ the child getting the vaccine: vaccinating children.

Hib vaccine schedule - ever had a serious allergic , : reaction or other problem. $\because$ Most children should have $\cdot$ currently has moderate or

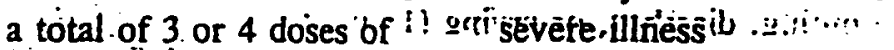
Hib vaccine. They should have Hib vaccine at: (zsis 1, a.:

If you are not sure, ask your $\checkmark 2$ months of age doctör öt nuturse."

$\checkmark 4$ months of age

$\checkmark 6$ months of age. This dose is not needed with some brands of vaccine.

$\checkmark$ 12-15 months of age.

Other vaccines may be given at the same time as Hib vaccine. 


\section{$\because$ Yi.j: :}

What are the risks from Hib vaccine?

Hib vaccine is :one of the safest of all vaccines.

$$
\begin{aligned}
& \text { : a . , , this: }
\end{aligned}
$$

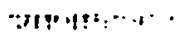

It has not been،shown. to cause any serious problems... . : $r: !^{\prime}$. …:

As with any medicine, there is a very small risk that serious problems, even death, could occur after getting a vaccine.

Getting the disease is much more likely to cause serious illness than getting the vaccine.
What to do if there is a serious reaction:

Call a doctor or get the child to a doctor right away.

0 Ask your doctor $r$ nurse, of health department to file a Vaccine Adverse Event Report form.

To file a report yourself you can call

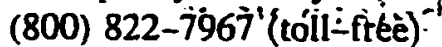

If you want to learin moré, ask your doctor or nurse. She/he can give you the vaccine package insert or suggest other sources of information.

\section{Mild problems}

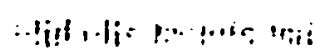

If these problems come, they usually start within 1 day of vaccination. They may last 2-3 days. They include:

redness i, warmth, or swelling where the" shot was given (1 out of every 4 doses's)

- fever over $101^{\circ}$ ( 1 out of every 100 doses)

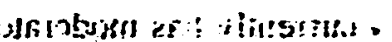

- vomiting, diarrhea, $: 9 r$, crying (1 out of of

$$
\text { every } 100 \text { doses) : }
$$

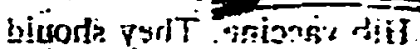

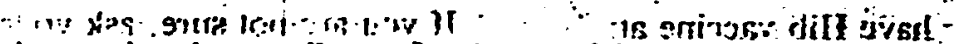
Acetaminophen or, ibuprofen (not aspirin) may be used to reduce fever and pain.

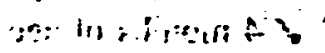
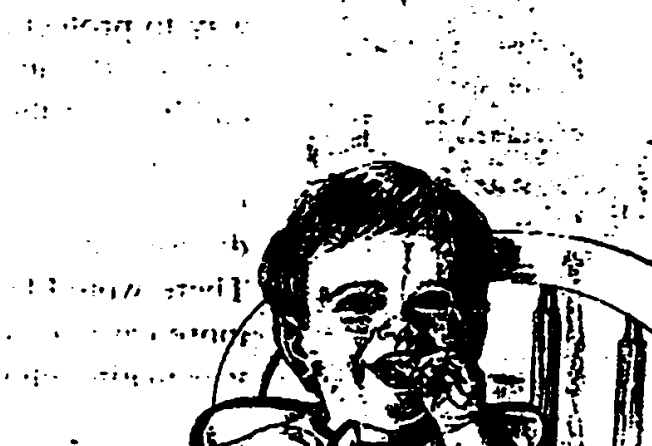

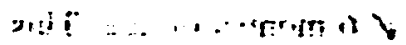

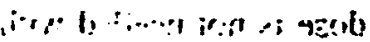

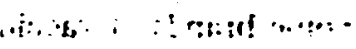

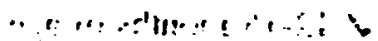

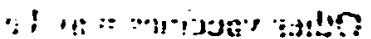

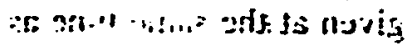

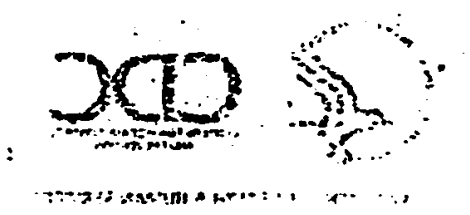

=rigeste rill 


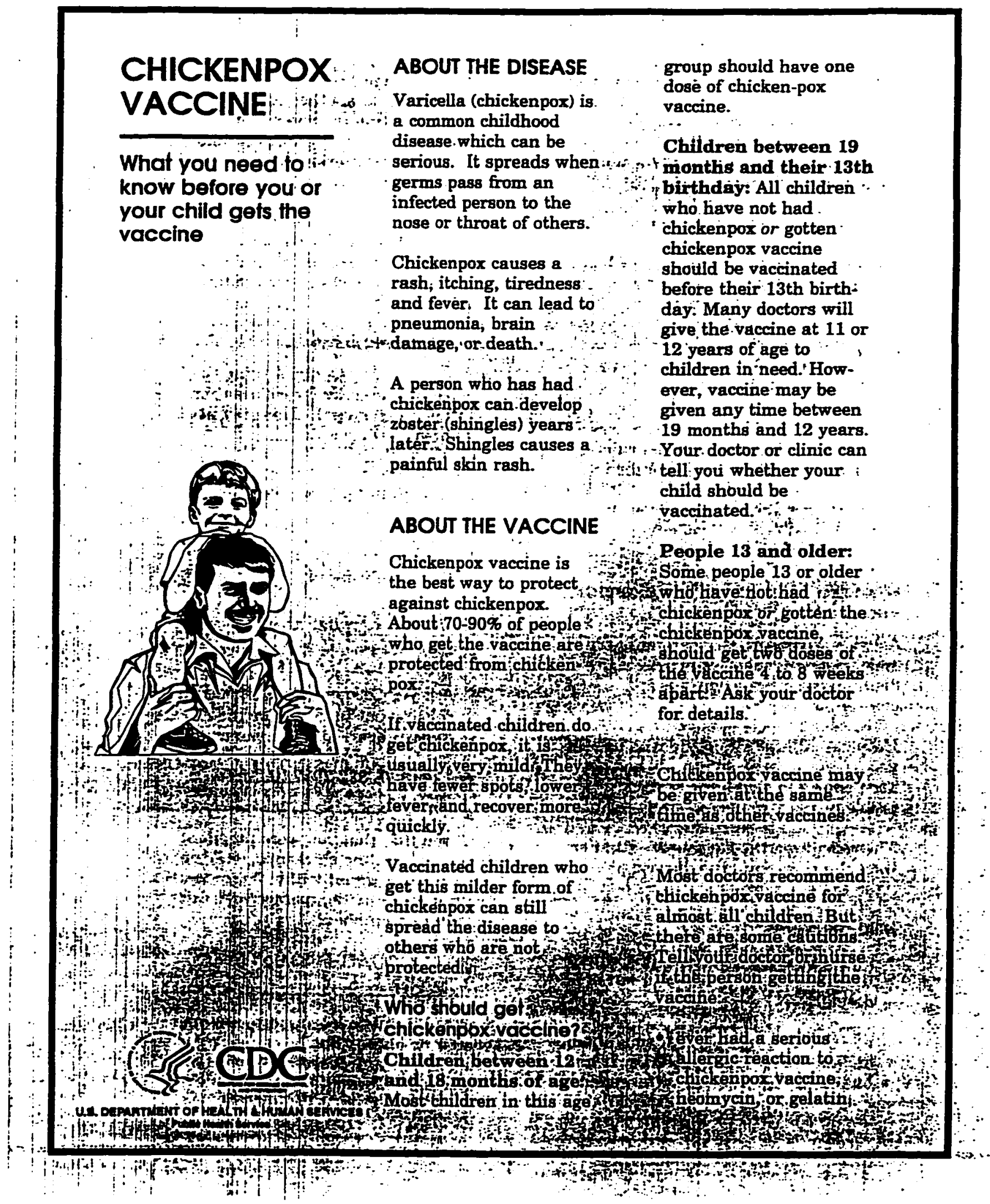


- now has a moderate or serious illness,

- is pregnant,

- is unable to fight serious.infections because, of:

$$
\because \therefore+1 \text { i }
$$

- a diseáse (like HIV/AIDS)

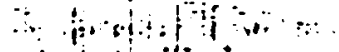

- treatment lwith drugs such as longterm steroids in

- any kind of cancer.

$$
\because \text { h. }
$$

i. - cancer treatment with x-rays or

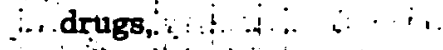

$$
\text { 20.?!" }
$$

has gotten blood products (such as immune globulin or a transfusion) - during the past several months.:

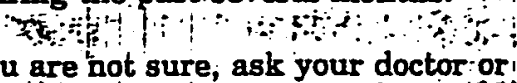

If you are not sure, ask your doctor or nurse

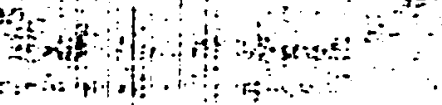

What are the risks.from chickenpox

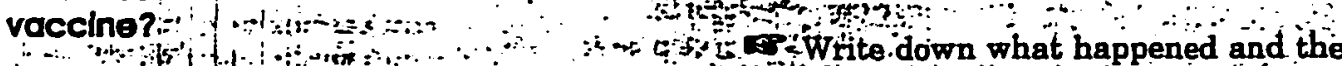
As with any medicine, there are very $\rightarrow$ of small risks that serious problems, even death, could occur after getting chickenpox vaccíne.

$$
\text { enpox vaccine. } \because \because \div:
$$

However, almost all people who use the vaccine have no problems. It has not been shown to cause any serious ptoblems.

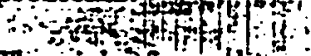

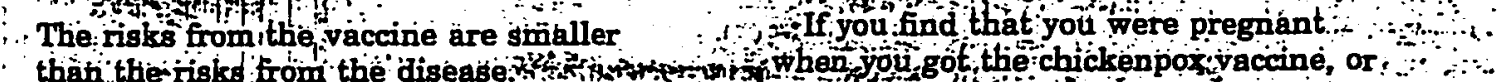

Modercite probiems problems

a seizure (jerking and staring spell) usually caused by fever (less than 1 out of 1,000 doses). This may not be related to the vaccine r y

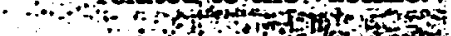

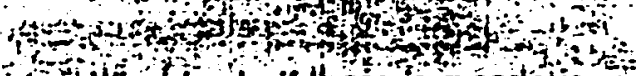
What to do if there is a sertotis Feadifion Call a doctor or get the person to a

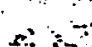
doctor right away.

\section{$\cdots$
$\therefore$
$\therefore$
$-\cdots$}

Ask your doctor nurse, or health departmerit: to file a $\vec{V}$ accine Adverse Event Report form br you can call a

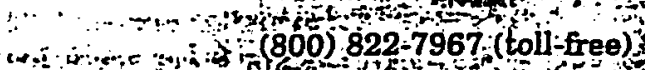
$\ldots$ than thenses trom the disease

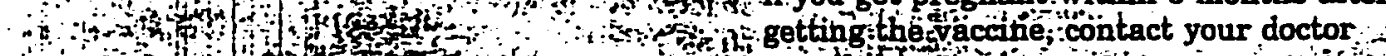

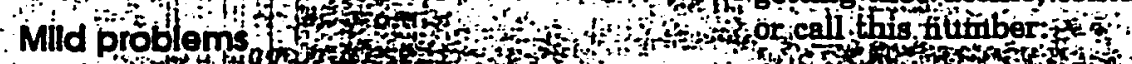

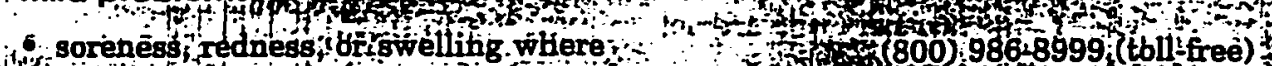

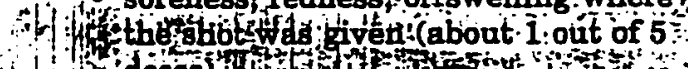
doses), The it doses,

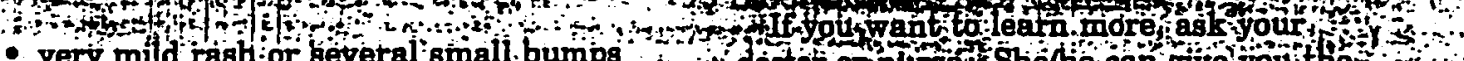

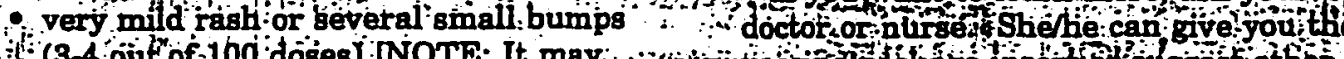

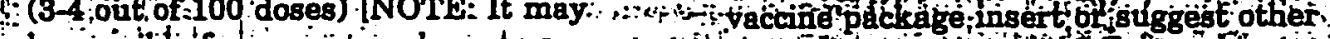

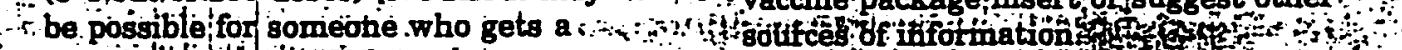

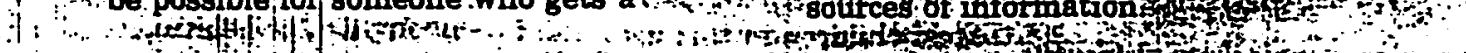

$i=\cdots$

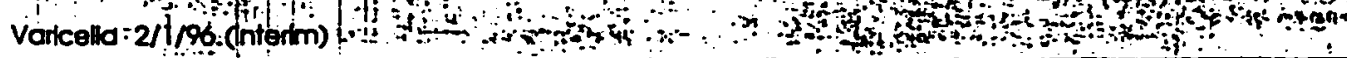

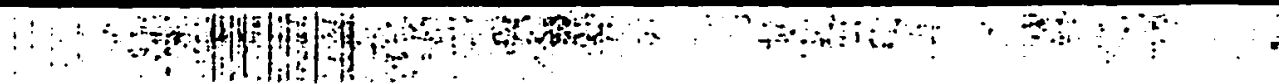


MEASLES,

MUMPS,

AND

RUBELLA

VACCINE

(MMR)

What you need to know before you or your child gets the vaccine
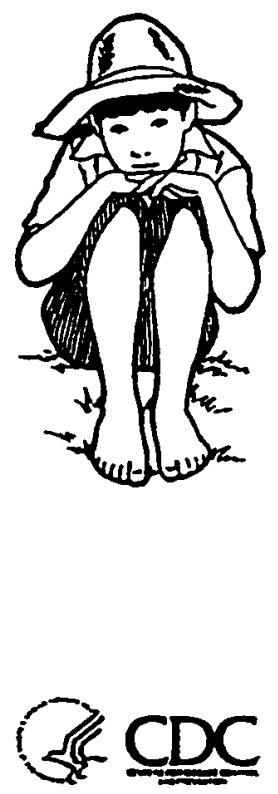

U.S DEPARTMENT OF REALTH \& HUUAN SERVICES Putele thelen Senteo Cortera bo Obesec Controt ins Prevertion

\section{ABOUT THE DISEASES}

Measles. mumps. and rubella (German measles) are serious diseases. They spread when germs pass from an infected person to the nose or throat of others.

\begin{tabular}{|c|c|c|}
\hline $\begin{array}{l}\text { Measies causes: } \\
\text { rosh } \\
\text { cough } \\
\text { fever }\end{array}$ & $\begin{array}{l}\text { Mumps causes: } \\
\text { fever } \\
\text { heodache } \\
\text { swollen glands under } \\
\text { the jow }\end{array}$ & $\begin{array}{l}\text { Rubella causes: } \\
\text { rash } \\
\text { mild fever } \\
\text { swollen glands } \\
\text { arthritts (mostly in } \\
\text { women) }\end{array}$ \\
\hline $\begin{array}{l}\text { If can lead fo: } \\
\text { - ear infection } \\
\text { - pneumonia } \\
\text { - dianhea } \\
\text { - seizures Jerking } \\
\text { and staring spells) } \\
\text { - brain domage } \\
\text { - death }\end{array}$ & $\begin{array}{l}\text { If can lead lo: } \\
\text { - hearing loss } \\
\text { - meninglitis (infection } \\
\text { of brain and spinal } \\
\text { cord coverings) } \\
\text { - Males can have } \\
\text { painful. swollen } \\
\text { testicles. }\end{array}$ & $\begin{array}{l}\text { Pregnant women can } \\
\text { lose their babies. } \\
\text { Bobles can be born } \\
\text { with birth defects } \\
\text { such as: } \\
\text { - deafness } \\
\text { - blindness } \\
\text { - heart dlsease } \\
\text { - broin damage } \\
\text { - other serious } \\
\text { problems }\end{array}$ \\
\hline
\end{tabular}

\section{ABOUT THE VACCINES}

\section{Benefits of the vaccines}

Vaccination is the best way to protect against measles. mumps. and rubella. Because most children get the MMR vaccines. there are now many fewer coses of these disecses. There would be many more cases if we stopped vaccinating children.

\section{MMR schedule}

Most children should have a total of 2 MMR vaccines. They should have MMR at:

$\checkmark$ 12-15 months of age

$\checkmark 4-6$ years of age or before middle school or junior high school

Other vaccines moy be given at the same time as MMR.

\section{Who should get MMR vaccine?}

Most doctors recommend that almost all young children get MMR vaccine. But there are some cautions. Tell your doctor or nurse if the person getting the vaccine is less able to fight serious infections becouse of:

- a disease she/he was born with

- treatment with drugs such as long-term steroids

- any kind of cancer

- cancer treatment with $x$-rays or drugs

Also:

- People with AIDS or HIV Infection usually should get MMR voccine.

- Pregnant women should wait until after pregnancy for MMR vaccine. 
MEASLES,

MUMPS,

AND

RUBELLA

VACCINE

(MMR)

What you need to know before you or your child gets the vaccine
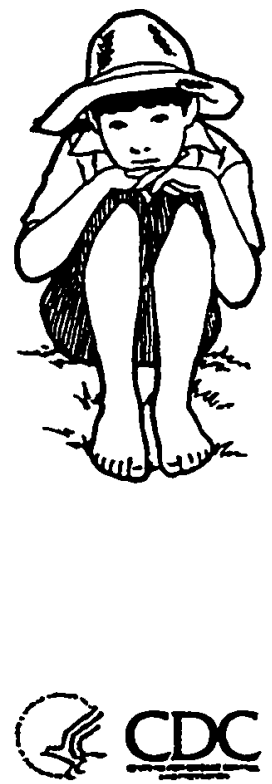

U.S. DEPAATLENT OF HEALTH \& HULAN SEAVMCES Putac Heath Sonto Conten bor Deveces Conted

\section{ABOUT THE DISEASES}

Measles. mumps, and rubella (German measles) are serious diseases. They spread when germs pass from on infected person to the nose or throat of others.

\begin{tabular}{|c|c|c|}
\hline $\begin{array}{l}\text { Meastes causes: } \\
\text { rosh } \\
\text { cough } \\
\text { fever }\end{array}$ & $\begin{array}{l}\text { Mumps couses: } \\
\text { fever } \\
\text { headoche } \\
\text { swollen glonds under } \\
\text { the jow }\end{array}$ & $\begin{array}{l}\text { Ruballa causes: } \\
\text { rash } \\
\text { mild fever } \\
\text { swollen glands } \\
\text { arthrttls (mostty in } \\
\text { women) }\end{array}$ \\
\hline $\begin{array}{l}\text { If can lead to: } \\
\text { - ear infection } \\
\text { - pneumonia } \\
\text { - diarrhea } \\
\text { - selzures (Jerking } \\
\text { and staring spells) } \\
\text { - broin damage } \\
\text { - death }\end{array}$ & $\begin{array}{l}\text { If can lead fo: } \\
\text { - hearing loss } \\
\text { - meningitis (infection } \\
\text { of brain ond spinal } \\
\text { cord coverings) } \\
\text { - Males can have } \\
\text { painful. swollen } \\
\text { iesticles. }\end{array}$ & $\begin{array}{l}\text { Pregnont women can } \\
\text { lose thelr babies. } \\
\text { Bobles can be born } \\
\text { with birth defects } \\
\text { such as: } \\
\text { - deatness } \\
\text { - blindness } \\
\text { - heart disease } \\
\text { - broin dornoge } \\
\text { - other serious } \\
\text { problems }\end{array}$ \\
\hline
\end{tabular}

\section{ABOUT THE VACCINES}

\section{Benefits of the vaccines}

Vaccination is the best way to protect against measles. mumps. and rubella. Because most children get the MMR vaccines. there are now many fewer cases of these diseases. There would be many more cases if we stopped vaccinating children.

\section{MMR schedule}

Most children should have a total of 2 MMR vaccines. They should hove MMR at:

$\sim$ 12-15 months of oge

$\checkmark$ 4-6 years of age or before middle school or junior high school

Other vaccines may be given at the same time as MMR.

\section{Who should get MMR vaccine?}

Most doctors recommend that almost all young children get MMR vaccine. But there are some coutions. Tell your doctor or nurse if the person getting the vaccine is less able to fight serious infections becouse of:

- a disease she/he was born with

- treatment with drugs such as long-term steroids

- any kind of concer

- concer treatment with $x$-roys or drugs

Also:

- People with AIDS or HIV Infection usually should get MMR voccine.

- Pregnant women should wait until affer pregnancy for MMR vaccine. 
- People with a serious allergy to eggs or the drug neomycin should tell the doctor or nurse. If you are not sure. ask the doctor or nurse.

Tell your doctor or nurse if the person getting the vaccine:

- ever had a serious allergic reactlon or other problem after getting MMR

- now has moderate or severe illness

- hos ever hod a seizure

- has a parent. brother. or sister who has had seizures

- has gotten immune globulin or other blood products (such as a transfusion) during the past several months

If you are not sure. ask your doctor or nurse.

\section{What are the risks from MMR vaccine?}

As with any medicine. there are very small risks that serlous problems. even death. could occur after taking a vaccine.

The risks from the vaccine are much smaller than the risks from the diseases if people stopped using voccine.

Aimost all people who get MMR have no problems from it.

\section{Mild or moderate problems}

- Soon after the vaccination. there moy be soreness. redness, or swelling where the shot was given.

- 1-2 weeks after the first dose. there may be:

- rash (5-15 out of every 100 doses)

- fever of $103^{\circ}$ or higher (5-15 out of every 100 doses). This usually lasts 1-2 doys.

- swelling of the glands in the cheeks. neck. or under the jow

- a seizure (jerking and staring spell) usually caused by fever. This is rare.

- 1-3 weeks after the first dose. there may be:

- pain. stiffness, or swelling in one or more joints lasting up to 3 doys ( 1 out of every 100 doses in children: up to 40 out of every 100 doses in young women). Rarely. pain or stiffness lasts a month or longer. or moy come and go: this is most common in young and adult women.
Acetaminophen or ibuprofen (non-aspirin) moy be used to reduce fever and soreness.

\section{Severe problems}

These problems happen very rarely:

- serious allergic reaction

- Low number of platelets (a type of blood cell) that can lead to bleeding problems. This is almost always temporary.

- long seizures. decreased consciousness. or coma

Problems following MMR are much less common after the second dose.

\section{What to do if there is a serious reaction:}

IS Call a doctor or get the person to a doctor right awoy.

Write down what happened and the date and time it hoppened.

Ask your doctor. nurse. or heaith department to file a Vaccine Adverse Event Report form or call:

(800) $822-7967$ (toll-free)

The National Vaccine Injury Compensation Program gives compensation (payment) for persons thought to be injured by vaccines. For details call:

(800) 338-2382 (toll-free)

If you want to learn more. ask your doctor or nurse. She/he con give you the voccine package insert or suggest other sources of information.

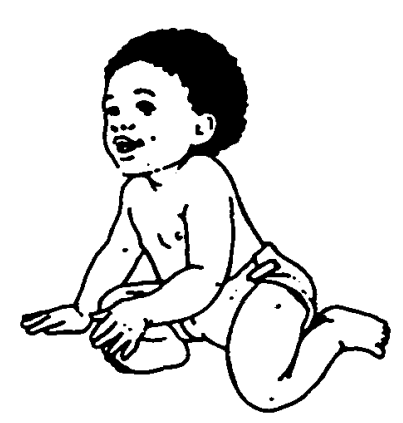




\section{Appendix I}

\section{University of San Diego Committee on the Protection of Human Subjects}

Approval November $26,1996 \ldots \ldots \ldots \ldots \ldots \ldots \ldots \ldots . \ldots \ldots$ 


\section{Appendix $\mathbf{J}$}

Results of t-tests for Differences between Vietnamese and Hmong Groups . . . . . 243 
Appendix J

Results of t-tests for Differences between Viemamese and Hmong Groups

\begin{tabular}{lrll}
\hline Variable & t-value & df & p value (2 tailed) \\
\hline Maternal Sensitivity & -.91 & 30 & .369 \\
PTS & -3.25 & 27 & .003 \\
Anxiety & -1.76 & 25 & .096 \\
Depression & -3.69 & 26 & .001 \\
Acculturation & .60 & 26 & .554 \\
\# Husb. Fam.+ & -1.06 & 29 & .299 \\
\# Pregnancies & -4.02 & 20 & .001 \\
Age & 1.42 & 25 & .169 \\
\# Yrs Transition & -4.33 & 15 & .001 \\
& & & \\
\hline
\end{tabular}

Note. PTS = Posttraumatic Stress; $+=$ Number of Husband's Family in the United States; 